A Il202 b23854

REFERENGE

NBS PUBLICATIONS

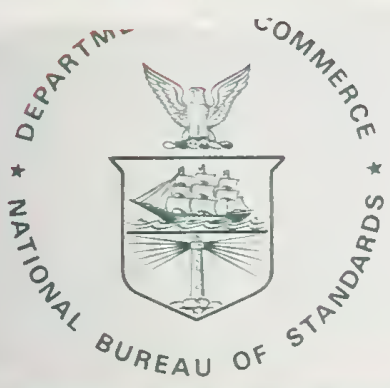

NBS SPECIAL PUBLICATION 400-74

U.S. DEPARTMENT OF COMMERCE/National Bureau of Standards

Semiconductor Measurement Technology:

\title{
Interlaboratory Study on Linewidth Measurements for Antireflective Chromium Photomasks
}

\section{$Q C$ \\ 100 \\ .457 \\ \#400-74}

1982 


\section{NATIONAL BUREAU OF STANDARDS}

The National Bureau of Standards' was established by an act of Congress on March 3, 1901. The Bureau's overall goal is to strengthen and advance the Nation's science and technology and facilitate their effective application for public benefit. To this end, the Bureau conducts research and provides: (1) a basis for the Nation's physical measurement system, (2) scientitic and technological services for industry and government, (3) a technical basis for equity in trade, and (4) technical services to promote public safety. The Bureau's technical work is performed by the National Measurement Laboratory, the National Engineering Laboratory, and the Institute for Computer Sciences and Technology.

THE NATIONAL MEASUREMENT LABORATORY provides the national system of physical and chemical and materials measurement; coordinates the system with measurement systems of other nations and furnishes essential services leading to accurate and uniform physical and chemical measurement throughout the Nation's scientific community, industry, and commerce; conducts materials research leading to improved methods of measurement, standards, and data on the properties of materials needed by industry, commerce, educational institutions, and Government; provides advisory and research services to other Government agencies; develops, produces, and distributes Standard Reference Materials; and provides calibration services. The Laboratory consists of the following centers:

\section{Absolute Physical Quantities ${ }^{2}$ - Radiation Research — Chemical Physics - Analytical Chemistry - Materials Science}

THE NATIONAL ENGINEERING LABORATORY provides technology and technical services to the public and private sectors to address national needs and to solve national problems; conducts research in engineering and applied science in support of these efforts; builds and maintains competence in the necessary disciplines required to carry out this research and technical service; develops engineering data and measurement capabilities; provides engineering measurement traceability services; develops test methods and proposes engineering standards and code changes; develops and proposes new engineering practices; and develops and improves mechanisms to transfer results of its research to the ultimate user. The Laboratory consists of the following centers:

Applied Mathematics - Electronics and Electrical Engineering ${ }^{2}$ - Manufacturing Engineering - Building Technology - Fire Research - Chemical Engineering ${ }^{2}$

THE INSTITUTE FOR COMPUTER SCIENCES AND TECHNOLOGY conducts research and provides scientific and technical services to aid Federal agencies in the selection, acquisition, application, and use of computer technology to improve effectiveness and economy in Government operations in accordance with Public Law 89-306 (40 U.S.C. 759), relevant Executive Orders, and other directives; carries out this mission by managing the Federal Information Processing Standards Program, developing Federal ADP standards guidelines, and managing Federal participation in ADP voluntary standardization activities; provides scientific and technological advisory services and assistance to Federal agencies; and provides the technical foundation for computer-related policies of the Federal Government. The Institute consists of the following centers:

Programming Science and Technology-Computer Systems Engineering.

'Headquarters and Laboratories at Gaithersburg, MD, unless otherwise noted; mailing address Washington, DC 20234.

${ }^{2}$ Some divisions within the center are located at Boulder, CO 80303. 
Semiconductor Measurement Technology:

\section{Interlaboratory Study on Linewidth Measurements for Antireflective Chromium Photomasks}

John M. Jerke

M. Carroll Croarkin

Ruth N. Varner

National Engineering Laboratory

National Bureau of Standards

Washington, DC 20234

Sponsored jointly by NBS and the:

Defense Advanced Research Projects Agency

1400 Wilson Blvd.

Arlington, VA 22209

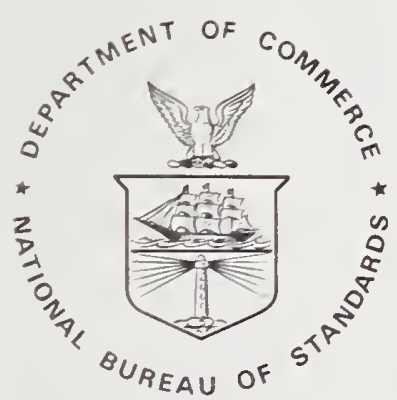

U.S. DEPARTMENT OF COMMERCE, Malcolm Baldrige, Secretary

NATIONAL BUREAU OF STANDARDS, Ernest Ambler, Director

Issued November 1982 
Library of Congress Catalog Card Number: 82-600642

National Bureau of Standards Special Publication 400-74 Natl. Bur. Stand. (U.S.), Spec. Publ. 400-74, 191 pages (Nov. 1982) CODEN: XNBSAV 
Preface

Abstract and Key Words . . . . . . . . . . . . . . . . . . . 1

1. Introduction . . . . . . . . . . . . . . . . . . . . . . 2

2. Description of study . . . . . . . . . . . . . . . . . . . 4 4

2.1 NBS Artifact . . . . . . . . . . . . . . . . . . . 4

2.2 NBS Measurement System . . . . . . . . . . . . . . . . 5

2.3 Procedures . . . . . . . . . . . . . . . . . . . . 6

2.4 Participants' Measurement Systems . . . . . . . . . . . . 8

3. Statistical Analysis . . . . . . . . . . . . . . . . . . 9

3.1 Approach . . . . . . . . . . . . . . . . . . 10

3.2 Graphical Analysis . . . . . . . . . . . . . . . . . 11

3.2.1 Individual Measurement systems . . . . . . . . . . . 11

3.2. 2 Youden Plots . . . . . . . . . . . . . . . . 12

3.3 Detection of Outliers . . . . . . . . . . . . . . . . 13

3.3.1 Isolated Outliers . . . . . . . . . . . . . . . 13

3.3.2 Entire Day's Measurements as Outliers . . . . . . . . 14

3.4 Calibration Curves . . . . . . . . . . . . . . . . 15

3.4.1 Line-Spacing Calibration . . . . . . . . . . . . . 16

3.4.2 Linewidth Calibration . . . . . . . . . . . . . . 18

3.5 Assessment of Line-Spacing and Linewidth Calibrations . . . . 19

3.5.1 Measurement Systems Combined . . . . . . . . . . . . 19

3.5.2 Individual Measurement Systems . . . . . . . . . . . . 20

3.5.3 Measurement Process Standard Deviation . . . . . . . 20

3.5.4 Uncertainty of Corrected Linewidth Values . . . . . . 20

3.6 Sources of Measurement Errors . . . . . . . . . . . . . . 21

3.6.1 Between-Day Differences in Calibration Curves . . . $\quad 21$

3.6.2 Linearity Assumption . . . . . . . . . . . . . . 23

3.6.3 Differences Between Operators . . . . . . . . . . . 24

3.7 Effects of Using Adjusted NBS Values . . . . . . . . . . . . 25

4. Discussion . . . . . . . . . . . . . . . . . . . . . 26

4. 1 NBS Recommended Procedures . . . . . . . . . . . . . . . 26

4.2 Linewidth Calibration Curves . . . . . . . . . . . . . . 27

4.3 Polarity Dependence of Linewidth Calibration . . . . . . . 27

4.4 Measurement Process Standard Deviation . . . . . . . . . . 28

4.5 Linearity of Calibration Curve . . . . . . . . . . . . . 29

5. Concluding Remarks . . . . . . . . . . . . . . . . . . 30

6. References . . . . . . . . . . . . . . . . . . . 32 
Appendix A - Design of a Pattern on a Photomask-Like Physical Standard for Evaluation and Calibration of Linewidth-Measuring

Systems (reprint) .............. 89

Appendix B - NBS Linewidth and Line-Spacing Values for Artifacts . . . 97

Appendix C - Recommended Procedures for Photomask Linewidth

Measurements (AR-Chromium) . . . . . . . . 9 98

Appendix D - Participants' Linewidth and Line-Spacing Measurements • . 137

Appendix E - Graphical Diagnosis of Interlaboratory Test Results

(reprint) . . . . . . . . . . . . 176

Appendix F - Calibration Curve from Method of Least Squares . . . . . 181

Appendix G - Standard Deviation of the Residual . . . . . . . . 183

LIST OF FIGURES

1. Photograph of overall pattern on NBS antireflective-chromium

artifact . . . . . . . . . . . . . . . 35

2. Photograph of central area of NBS antireflective-chromium

artifact . . . . . . . . . . . . . . . . 36

3. Photograph of basic measurement pattern on NBS antireflective-

chromium artifact . . . . . . . . . . . . . . . 37

4. Schematic of physical profile of opaque line on NBS antireflectivechromium artifact . . . . . . . . . . . . . . 38

5. Examples of differences between participant's linewidth measurements and NBS linewidth values as a function of NBS values . . . 39

6. Youden plots of differences between participant's raw linewidth measurements on opaque lines (rows A and $C$ ) and NBS values.. .41

7. Youden plots of differences between participant's raw linewidth measurements on clear lines (rows B and D) and NBS values . . . . 51

8. Youden plots of differences between participant's corrected linewidth measurements on opaque and clear lines (rows C and D) and NBS values . . . . . . . . . . . . 61

9. Daily changes in linear linewidth calibration curve for one

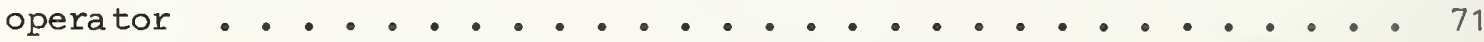

10. Dependence of systematic linewidth measurement errors on line polarity . . . . . . . . . . . . . . . . . 72 
11. Residual linewidth measurement errors of video image-scanning measurement system . . . . . . . . . . . . . . . . .

1. Uncertainty Assigned to NBS Linewidth Measurements for Interlaboratory Study . . . . . . . . . . . . . . . .

2. Measurement Systems Used by Participants in Interlaboratory Study . . . . . . . . . . . . . . . . . . . . .

3. Codes for Identifying Participants' Measurement Systems in

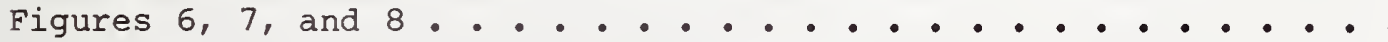

4. Least-Squares Estimates of Intercept $\alpha$ and Slope $\beta$ for LineSpacing Calibration Curves and Student's t Statistics . . . . . .

5. Least-Squares Estimates of Intercept $\alpha$ and Slope $\beta$ of Linewidth Calibration Curves and Student's $t$ Statistics for Opaque Lines (Row A) and Clear Lines (Row B) . . . . . . . . . . . . .

6. Frequency of Differences Between Participant's Linewidth Measurements and NBS Values on Opaque Lines (Row C) . . . . . .

7. Frequency of Differences Between Participant's Linewidth Measurements and NBS Values on Clear Lines (Row D) . . . . . .

8. Random Error Limits for Linewidth Measurements Corrected by Linear Calibration Curve, Process Standard Deviations, and Degrees of Freedom . . . . . . . . . . . . . . . .

9. F Statistics for Detecting Between-Day Differences in Linewidth Calibration Curves, Degrees of Freedom, and Pooled Standard Deviations from Linear Fit . . . . . . . . . . . . . .

10. Standard Deviations of Repetition, Process Standard Deviations, Degrees of Freedom, and F Statistics for Testing Linearity of Linewidth Calibration Model . . . . . . . . . . . . .

11. Frequency of Differences Between Participant's Linewidth Measurements and NBS Values for Two Operators on Each of Three Measurement Systems . . . . . . . . . . . . . . . . . 
This National Bureau of Standards (NBS) study was initiated in the Optics and Micrometrology Section, Mechanics Division, in cooperation with the Optical Physics Division, as a part of the Semiconductor Technology Program based in the Electronic Technology Division. Related work continues in the Semiconductor Materials and Processes Division, one of the two Divisions comprising the Semiconductor Technology Program at the present time. This Program serves to focus NBS research on improved measurement technology for the use of the semiconductor device community in specifying materials, equipment, and devices in national and international commerce, and in monitoring and controlling device fabrication and assembly. This research leads to carefully evaluated, well-documented test procedures and associated technology which, when applied by the industry, are expected to contribute to higher yields, lower cost, and higher reliability of semiconductor devices and to provide a basis for controlled improvements in fabrication processes and device performance. By providing a common basis for the purchase specifications of Government agencies, improved measurement technology also leads to greater economy in Government procurement. Financial support of the Program has been provided by a variety of Federal agencies: The Defense Advanced Research Projects Agency; The National Bureau of Standards; The Division of Electric Energy Systems, Department of Energy; The Defense Nuclear Agency; The C. S. Draper Laboratory; The Naval Air Systems Command; The Air Force Wright Aeronautical Laboratories; The Army Electronics Technology and Devices Laboratory; The Naval Weapons Support Center; The Solar Energy Research Institute; The Naval Avionics Center; The Lewis Research Center, National Aeronautics and Space Administration; The Office of Naval Research; and The Naval Ocean systems Center. The specific work reported herein was supported jointly by DARPA and $\mathrm{NBS}^{\dagger}{ }^{\dagger}$

This study depended on the cooperation of ten companies which are part of the U.S. microelectronics industry. These companies provided the personnel, facilities, and time necessary to make the measurements according to NBS procedures, return the data and artifacts to NBS, and respond to follow-up inquiries from NBS during the data analysis. For these efforts, special thanks are gratefully made to these ten companies and to the individual operators who participated in the study.

Special acknowledgment is gratefully made to the following NBS staff: Arie W. Hartman and Fred $W$. Rosberry for measuring linewidths and line spacings on the ten artifacts used in this study; Dr. Dennis A. Swyt for analysis of the NBS measurement data and for his contributions to the design of the artifact used in this study; william $\mathrm{R}$. Smallwood ${ }^{\dagger}$ for remeasurements of the artifacts following their return from industry; Dr. Diana Nyyssonen and Dr. W. Murray Bullis" for their helpful suggestions in the design of the study and the analysis of the results; Dr. James J. Filliben for making alterations and additions to the computer software used to plot the results; and Dr. Clifford H. Spiegelman and Dr. Keith R. Eberhardt for their helpful advice on the statistical analysis. Special acknowledgment is also made to the NBS Office of Measurement Services which supported part of this study.

\footnotetext{
† Through ARPA Order 2397.

$\S$ Retired.

I Present address: Fairchild R\&D Laboratories, Palo Alto, CA.
} 


\section{Disclaimer}

Certain commercially available materials or instruments are identified in this publication for the purpose of providing a complete description of the work performed. The experiments reported do not constitute an evaluation of the performance characteristics of the products so identified. In no case does such identification imply recommendation or endorsement by the National Bureau of Standards nor does it imply that the items identified are necessarily the best available for the purpose. 
Semiconductor Measurement Technology:

Interlaboratory Study on Linewidth Measurements

for Antireflective Chromium Photomasks

John M. Jerke

Semiconductor Materials and Processes Division

Center for Electronics and Electrical Engineering

M. Carroll Croarkin and Ruth N. Varner

Statistical Engineering Division

Center for Applied Mathematics

National Bureau of Standards

Washington, DC 20234

Abstract: Optical microscopes fitted with a micrometer attachment are commonly used to measure small linewidths and other critical dimensions on integrated-circuit (IC) photomasks. In the absence of calibrated linewidth standards, users have experienced systematic measurement errors much larger than required manufacturing tolerances. As IC linewidths approach 1 im with a 10 percent tolerance, the need for calibration standards and improved measurement procedures becomes even more important.

This report discusses the results of an interlaboratory study to evaluate a National Bureau of Standards (NBS) prototype calibration standard for linewidth measurements on IC photomasks and procedures for adjusting and calibrating optical-microscope systems in the $0.5-$ to $12-\mu \mathrm{m}$ measurement range. Using procedures furnished by NBS, industrial participants measured line spacings and linewidths on NBS antireflective-chromium artifacts. A comparison of NBS linewidth values with participants' measurements showed that most differences were less than $\pm 0.3 \mu \mathrm{m}$. A linewidth calibration significantly reduced systematic errors for most systems. Outliers in the data showed the need for measurementcontrol procedures. The standard deviation of the measurement process was $\pm 0.1 \mathrm{~m}$ or larger for about half of the systems. For some systems, there were significant day-to-day differences and operator differences.

The study showed that the NBS artifact and recommended procedures were adequate for calibrating an optical-microscope system which was in a state of statistical control. This study led to the issuance of NBS Standard Reference Material 474 (Optical Microscope Linewidth-Measurement Standard).

Key words: dimensional measurements; filar micrometer; imageshearing micrometer; integrated circuits; interlaboratory study; Kohler illumination; line-spacing measurements; linewidth calibration; linewidth measurements; measurement uncertainty; micrometrology; optical microscope; photomask; semiconductor technology; statistical tests. 


\section{INTRODUCTION}

Optical imaging systems which use an optical microscope fitted with some type of measurement attachment are commonly used for linewidth measurements in the 0.5 - to 12- $\mathrm{m}$ range on integrated-circuit (IC) photomasks. Differences as large as $1 \mathrm{\mu m}$ for linewidth measurements on the same photomask exist for different types of optical imaging systems, different systems of the same type, different operators on the same system, and measurements made by the same operator at different times of the day. Such differences are, for example, much larger than the required tolerances of 10 percent or less on 1to 2- $\mu \mathrm{m}$ wide lines for Very Large Scale Integration (VLSI) geometries. Although some of these differences can be attributed to manufacturing tolerances or human errors, a much larger source of measurement differences is the diversity of methods used by these systems for detecting line edges and determining linewidth [1].

The term linewidth, as used in semiconductor technology, has traditionally been defined in a variety of ways depending upon its specific application. For example, the width of a line on a photomask used in photolithographic projection printing is defined differently from the width of a photoresist window used for etching a sublayer, or the width of a conducting line used for electrical measurements of an IC device. In the present case, linewidth is defined, at a given cross section of the line, as the distance between the air/line-material boundaries at some specified height above the interface between the patterned layer in which the line is formed and the underlying layer. The physical basis for various applications or methods of measuring linewidth may result in measurements being made at different heights above the interface. For this reason, differences may be expected between measurements made optically, electrically, or with a scanning electron microscope. Only optical measurements are considered in this report.

In an effort to provide the microelectronics industry with calibration standards and procedures for determining and reducing systematic linewidthmeasurement errors, the National Bureau of Standards (NBS) undertook an effort in late 1974 to develop a linewidth standard and procedures for using this standard with different types of measurement systems [2]. A pilot interlaboratory study involving NBS and three companies was conducted in mid1977 to determine the suitability of a chromium-on-glass artifact as a linewidth standard, to evaluate NBS recommended measurement procedures as a protocol for making linewidth measurements, and to elicit comments from the participants for possible improvements in the standard and procedures [3] This study showed linewidth-measurement differences as large as $0.5 \mathrm{~mm}$ on a

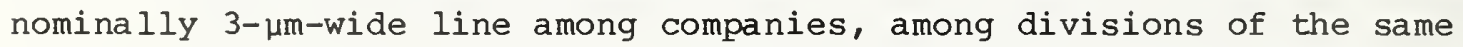
company, and among groups of the same division of the same company. The results of this relatively modest pilot study were subsequently used as a basis for redesigning the artifact [4] and revising the measurement procedures.

A full-scale interlaboratory study involving NBS and ten companies was conducted in 1978-79 to evaluate the revised linewidth-measurement procedures and to determine the suitability of the redesigned antireflective chromium-on-glass artifact as an NBS linewidth standard. In addition, this interlaboratory study was designed to enable organizations using optical- 
linewidth measurement systems to determine their relative measurement capability and the degree of improvement they can expect from the use of a calibrated linewidth standard and accompanying measurement procedures.

This report presents the results of the full-scale interlaboratory study. Section 2 of the report provides a description of the NBS artifact, the specified measurement procedures, and the measurement systems used by the participants in the study. Section 3 is a statistical analysis of the data provided by the participants and includes consideration of outliers, derivation of the calibration curves, and a discussion of the sources of measurement errors. Section 4 uses the results of the statistical analysis in section 3 to address several questions about the artifact, the measurement procedures, and the measurement systems used by the participants, including the effects of applying a linewidth calibration curve to reduce systematic errors, the dependence of systematic errors on the line polarity (clear or opaque), and the effect of the measurement process standard deviation on the linewidth calibration curve. Section 5 summarizes the important results of this study.

The appendices include both data and reference material required for a more complete understanding of this study and the resulting conclusions. Appendix $A$ is a reprint of an article describing the basis for the design of the artifact used in this study. Appendix B gives the NBS measurements for all of the artifacts. Appendix $C$ is the set of instructions sent to each participant, and Appendix D gives the raw data received from each. Appendix $\mathrm{E}$ is a reprint describing the method of Youden plots used in the analysis of the data. Appendices $F$ and $G$ give the mathematical derivation of the calibration curves and standard deviation of the residuals, respectively. 


\section{DESCRIPTION OF STUDY}

Each of the ten participating companies received from NBS one antireflective (AR) chromium artifact ${ }^{\dagger}$, procedures for adjusting the measurement systems and making measurements, a wratten 60 (green) filter $\$$, and data sheets. In contrast with the earlier blind pilot study in which industrial participants adjusted their measurement systems by their own techniques and standards, participants in the full-scale study were asked to follow only those procedures recommended by NBS and to use only the NBS-furnished line-spacing values and line-to-space ratio for adjusting their measurement systems.

The industrial participants in the interlaboratory study included manufacturers of photomasks, ICs, and linewidth-measurement equipment. In some companies, more than one division or group participated in the study. In these cases, each division or group received a separate set of instructions and data sheets, but measured the same artifact.

Each participating group used one or more linewidth-measurement systems representative of those commonly used for critical dimensional measurements in the IC industry.

The present study was restricted to measurements in bright-field transmitted light. It is preferred that linewidth measurements on photomasks be made in transmitted light. In reflected light, the line-image quality is degraded because of the strong wavelength dependence of the relative phase and contrast at the line edges and the poor (compared to transmitted-light measurements) signal-to-noise ratio for most specimens [5]. To reduce the image blurring due to this wavelength dependence would require the use of a filter with a much narrower spectral bandwidth than that of the wratten 60 filter used in this study.

\subsection{NBS Artifact}

The nine artifacts used in the study were made from AR-chromium photoplates by conventional photolithographic techniques. The substrate was soda-lime glass nominally 6.35 by 6.35 by $0.15 \mathrm{~cm}(2.5$ by 2.5 by $0.060 \mathrm{in.})$. The nominal thickness of the two-part AR-chromium layer was $120 \mathrm{~nm}$.

Figure 1 shows the overall pattern of the artifact. The cross-shaped clear area contains a series of horizontal and vertical lines which are used to help locate the basic measurement pattern. The basic pattern is repeated at. eight locations around the center of the artifact as shown in figure 1. Figure 2 is a magnified view of the central area of the artifact. Each basic pattern is located near the end of a horizontal or vertical line or both. An underlined number ( 1 through 8 ) is located next to each basic pattern. These numbers were used to identify which one of the eight basic patterns was to be measured in the study.

Figure 3 shows the basic measurement pattern on the artifact. Letters $A$ through $\mathrm{F}$ designate the row location, and numbers 0 through 9 designate the

† Two of the ten companies used the same artifact at different times. $\S$ See disclaimer, p. vii. 
column location for each line, line pair, or other pattern element; for example, A-6 refers to the opaque line in row $A$ and column 6 . Rows $A$ and $C$ contain opaque lines on a clear background, and rows $B$ and $D$ contain clear lines on an opaque background. These opaque and clear lines are used for calibrating the optical microscope to measure linewidths of both polarities (opaque and clear). In each of these rows there are ten lines with nominal widths from 0.5 to $12 \mu \mathrm{m}$ randomly distributed in the row. Row $\mathrm{E}$ consists of opaque line pairs (two interior lines of equal length) for calibrating the optical microscope to measure line spacing or pitch. The nominal line spacings of these line pairs are from 2 to 11 w randomly distributed in the row. Row $F$ consists of adjacent lines and spaces (two interior lines of equal length and intervening space) of approximately equal widths for setting the line-to-space ratio on video image-scanning micrometer attachments. The rationale for the design of this artifact is discussed in Appendix A [6].

\subsection{NBS Measurement System}

The artifacts were originally measured at NBS on a scanning opticalmicroscope system that was designed at NBS and is considerably different from any measurement system used by participants in this interlaboratory study. This NBS system is described in reference 4.

Two trained NBS operators measured a total of ten artifacts. After shipment from NBS, one artifact developed persistent contamination and was not used in this study. Four measurements were made of each linewidth in rows A, B, C, and $D$ and of each line spacing in row $E$. The mean values of the four measurements for each of these patterns on the nine artifacts used in the study are given in Appendix B. The widths of an opaque line and the adjacent clear space in pattern F-5 were also measured. This line-to-space ratio for F-5 and the line-spacing value for pattern E-5 were furnished to the participants for adjusting their measurement systems. (See sec.2.3.)

After the artifacts were measured on the NBS scanning optical-microscope system and shipped to the participants, another NBS scanning opticalmicroscope system with several improvements became operational. An intercomparison between the improved system and the system originally used to measure the artifacts showed that the latter system exhibited significant flare or scattered light. Furthermore, other developments showed the need to include a correction for the optical-phase difference at the line edge in the coherent edge-detection algorithm used for linewidth measurements on ARchromium photomasks [7]. Therefore, remeasurements of the artifacts were made with the improved system following the study. A comparison of the remeasurements with the original measurements showed that the flare present in the system used to make the original measurements and the exclusion of the correction for optical-phase difference in the original measurements introduced a larger systematic error in the original NBS linewidth values than was first estimated. (See sec. 3.7.)

The uncertainties assigned to the original NBS linewidth measurements on the artifacts are given in table 1. The systematic component due to line-edge detection is an approximation of the systematic error in relating the exact position along the edge profile of the material line to the transmittance 
threshold at which the scanning optical-microscope system detects the line edge. The value of $\pm 0.05 \mathrm{um}$ for this systematic error is a conservative estimate based on a line profile with a 70 deg line-edge slope and a $120 \mathrm{~nm}$ thickness as shown in figure 4. The other systematic component in table 1 includes systematic errors due to flare and the absence of a correction for optical-phase difference in the original linewidth measurements. Because flare is greater for an opaque line on a clear background than the reverse, this systematic component is slightly larger for rows $A$ and $C$. The overall linewidth-measurement uncertainty can be estimated by adding in quadrature all of the systematic uncertainty components and the random error component. Using this approach and the error components given in table 1, the overall uncertainty for the NBS linewidth value is given by

$$
\sqrt{(0.05 \mu \mathrm{m})^{2}+(0.05 \mu \mathrm{m})^{2}+(0.10 \mu \mathrm{m})^{2}} \simeq \pm 0.12 \mathrm{~mm}^{\dagger}
$$

\subsection{Procedures}

The recommended linewidth-measurement procedures furnished to each participant are reproduced in Appendix C. The procedures include specimen handling, equipment selection, microscope setup, method of measurement and data taking, and instructions for returning the data and artifact to NBS. The procedures also provide explanatory notes and background information on some of the procedural steps. The participants were invited to write any comments on these procedures directly in the procedures booklet and return it to NBS.

In selecting measurement equipment for the study, participants were asked to choose a microscope objective with a numerical aperture of 0.65 or larger and a condenser with a numerical aperture two-thirds that of the objective. The relatively high numerical aperture objective is necessary to resolve micrometer and submicrometer-wide lines on the artifact. The two-thirds ratio of condenser to objective numerical aperture provides an image with steep edge gradients while maintaining image brightness [8]. As a result, the repeatability of edge settings used in measurement eyepieces and photometric line profiles is improved.

Participants were asked to adjust the microscope for Kohler illumination which provides uniform illumination of the specimen, reduces stray light, and results in bright images with good contrast. After adjusting for Kohler illumination, the participants were asked to check the resolving power of the objective by observing a pair of nominally $1-\mu m$-wide lines on the artifact (pattern E-2). (See fig. 3.) Instructions were also provided for obtaining best focus by visual focusing either through the microscope or on the face of the cathode ray tube in video systems.

+ By comparison, the overall uncertainty currently assigned to NBS Standard Reference Materials 474 and 475 (Optical Microscope Linewidth Measurement Standards), which are measured on an improved version of the scanning optical-microscope system, is only $\pm 0.05 \mu \mathrm{m}$. The dominant contribution to this uncertainty is the estimated maximum systematic error resulting from the finite material edge slope. The estimated random error component is less than $\pm 0.02 \mathrm{um}$ (three sigma). Corrections are now made for both flare and optical-phase difference at the line edge. 
Participants were asked to place the broadband Wratten 60 green filter supplied by NBS between the light source and condenser. The use of such a spectral filter is intended to improve image quality. Optical microscopes are generally designed for optimum performance at or near a wavelength of 530 nm. Therefore, with white-light illumination, the imaging optics will typically exhibit chromatic aberrations. Studies have shown significant differences between optical line-edge profiles for white light and filtered green light [9].

Participants were asked either to adjust their instruments to read directly the value of an NBS-furnished length scale on the artifact (pattern E-5) or to determine a length-scale calibration factor based on the NBS value and correct their linewidth measurements by this factor. Those participants who used a video image-scanning system were also asked to adjust the system until the ratio of the width of a designated opaque line to the width of the adjacent clear line on the artifact (pattern $\mathrm{F}-5$ ) was equal to the NBSfurnished ratio. This adjustment corrects for linewidth errors due to an incorrect electronic edge-detection threshold introduced by the video processing.

Additional detailed instructions were provided to the participants for making measurements with some types of micrometer attachments. For example, participants using an image-shearing measurement system were asked to make linewidth measurements in a double-shear mode and line-spacing measurements in a modified single-shear mode. For other types of measurement systems, such as those with video and optical image-scanning micrometer attachments, the instructions were general and intended only as guidelines. Such measurement systems are usually either custom designed or the operator controls vary considerably between types and models; therefore, it is difficult to provide specific instructions for each of these systems.

A sample of the information and data sheets given to the participants is shown in Appendix C, Attachment F. Participants were asked to fill out pertinent information about their companies and their measurement equipment. This information was later used by NBS to determine if the participant's equipment had met the specifications required for the study. Participants were also asked to enter the date and time at the beginning and end of each set of measurements.

Participants were asked to assign to the study two trained operators who made linewidth measurements as part of their routine inspection of photomasks or as part of the manufacture and inspection of measurement systems or dimensional standards. Each of the two operators was asked to make four independent sets of measurements on each measurement system to give a total of eight sets of measurements for each system. Operators were asked to start from the beginning of the recommended procedures for each set of measurements and to arrange their schedule so that each operator made only one set of measurements each day. (See Appendix C, Attachment G for the suggested schedule.) For each set of measurements, the operator was asked to measure all ten linewidths in each of the rows $A, B, C$, and $D$ and all ten $l$ ine spacings in row $\mathrm{E}$. (See fig. 3.) 
The recommended procedures stressed the importance of making one set of measurements at one setting and making no changes to the measurement system, except focusing and moving the artifact, between the first and last

measurement. If any other changes were made, the operator was asked to start over from the beginning of the recommended procedures.

\subsection{Participants' Measurement Systems}

The measurement systems used by the participants in the study were basically an optical microscope to which any one of a variety of micrometer attachments was added [10]. The five types of micrometer attachments used in the study were: (1) optical filar, (2) video filar, (3) image shearing with optical display, (4) optical image scanning, and (5) video image scanning. Table 2 lists the measurement systems used by the participants. Data from some measurement systems were not analyzed by NBS because either the participant did not furnish all the requested data or did not follow directions. An alphanumeric code shown in table 2 is used to identify those systems for which data analyses are presented. 


\section{STATISTICAL ANALYSIS}

In the earlier pilot study involving NBS and three companies, the differences between NBS linewidth values and linewidth measurements made by industry using commercially available optical-microscope systems were largely systematic [11]. In-house NBS studies also showed the presence of systematic differences between NBS values and linewidth measurements made on several types of measurement systems widely used in the IC industry [12]. Analysis of optical imaging in a microscope has shown that these systematic errors in linewidth measurements are due, in large part, to using either an improper measuring scale or an optical threshold that does not correspond to the true line-edge location or both $[13,14]$. An additional systematic error may arise as the linewidth approaches the optical resolution of the system and the line image takes on the characteristics of the impulse response of the imaging system [15].

In the absence of a linewidth standard, users of linewidth-measurement systems have commonly taken the approach of using a line-spacing standard to calibrate their systems for linewidth measurements [16]. An in-house NBS study suggested that all of the systematic linewidth errors could not be eliminated by using this line-spacing calibration [17]. The in-house study did show, however, that systematic errors could be virtually eliminated by the use of a standard with both calibrated line spacings and linewidths.

The earlier pilot study involved measurements at only three points over the 1 to $10 \mu \mathrm{m}$ range and indicated that for some systems the observed linewidths were not a linear function of NBS values at linewidths approaching $1 \mu m$ [18]. This nonlinear behavior may have been due to the limited optical resolution of these measurement systems. Other common causes of nonlinear behavior are worn screw threads in the micrometer attachment, optical distortion in the imaging optics, and distortion in the cathode ray tube of video systems. A linewidth calibration curve based on a few linewidths would not be sufficient for measurement systems that show a nonlinear relationship between the observed linewidths and the NBS values.

The revised artifact [19] used in the present interlaboratory study was designed to overcome some of the limitations encountered in these earlier studies. The revised artifact contained a variety of linewidths sufficient for determining whether or not the observed linewidth values were a linear function of NBS values over the 0.5 to $12 \mu \mathrm{m}$ range. The artifact also contained a variety of line spacings for determining the measuring-scale errors separately from other types of systematic errors. The artifact was also designed to determine the extent to which systematic linewidth errors could be eliminated by using a linewidth calibration. For this purpose, the artifact had two sets of both clear and opaque lines. One set (rows $A$ and B) was used to establish the calibration curves for each type of line. The other set (rows C and D) was used as the "unknown" lines to be measured and subsequently corrected by the linewidth calibration. (See fig.3.)

Results from earlier studies also showed that systematic linewidth errors may be masked by a large scatter in the data due to differences in focus settings, poor image quality, and inability to repeat edge settings [20]. Therefore, the procedures used in the present interlaboratory study were 
designed to help control the linewidth-measurement process so that, to the greatest extent possible, a repeatable relation existed between the line-edge location and the edge-detection threshold.

The analysis of the data from the present interlaboratory study was designed to determine for each measurement system the magnitude of the random and systematic linewidth-measurement errors, the feasibility of a linear linewidth calibration curve, and the effectiveness of the NBS artifact and recommended procedures for eliminating measurement errors.

\subsection{Approach}

The basic approach used in the statistical analysis of the data from the interlaboratory study was first to use the differences between the participant's measurements and the NBS values on rows A (opaque lines), B (clear lines), and $\mathrm{E}$ (line spacings) for deriving linear linewidth calibration curves, and second to determine to what extent the application of these calibration curves eliminated any systematic errors in linewidth measurements made by the participants on rows $C$ (opaque lines) and D (clear lines). (See fig. 3.) Many of the statistical concepts and procedures used in this analysis are discussed in reference 21.

The following questions were addressed in the statistical analysis:

1. Are the participants in agreement with each other and with NBS in measuring linewidths on the artifacts or are there systematic differences among the participants and between NBS and each participant? (See sec. 3.2.)

2. How often are data outliers occurring that are large enough to perturb the results? (See sec. 3.3.)

3. If systematic linewidth errors exist, can a correction based solely on a linear line-spacing calibration eliminate these errors? (See sec. 3.4.1.)

4. If a line-spacing calibration is not sufficient for eliminating all systematic errors, can a correction based on a linear linewidth calibration that uses ten linewidths on the artifact eliminate these systematic errors? (See sec. 3.4.2.)

5. What are the measurement process precision (random error) and the measurement uncertainty of linewidths that are corrected based on the derived linewidth calibration curve? (See sec. 3.5.3.)

6. Are the measurement systems sufficiently stable so that the same linewidth calibration curve can be used on a day-to-day basis? (See sec. 3.6 .1$.

7. Are the participant's linewidth measurements a linear function of NBS values? (See sec. 3.6.2.)

8. Are there operator differences within each company? (See sec. 3.6.3.) 


\subsection{Graphical Analysis}

\subsubsection{Individual Measurement Systems}

As a first step in analyzing the data from the interlaboratory study, the differences between the participant's measurements ${ }^{\dagger}$ and NBS values (participant's measurement minus NBS value) for each measurement system were plotted against the NBS values for rows A, B, C, D, and E. Examples of these difference plots are shown in figure 5. The numbers on the plots indicate different days on which the artifact was measured, and each day's measurements are connected by solid lines. If all eight measurement repetitions were completed, there are eight curves for each measurement system. However, the numbers on the curves do not necessarily represent the order in which the measurements were taken. The ordering is arranged so that curves 1 through 4 are for the data from the first operator in chronological order, and curves 5 through 8 are the data from the second operator in chronological order. The same pattern is followed for fewer than eight repetitions, e.g., in the case of six repetitions, curves 1 through 3 are the data from the first operator and curves 4 through 6 are the data from the second operator.

Ideally all the data points in a difference plot of the type shown in figure 5 should be scattered randomly along the $y=0$ line (differences from NBS are plotted on the $y$-axis), thereby indicating negligible systematic error. Obviously the maximum spread among the data points must also be sufficiently small for the intended purpose of the system.

In the nonideal situation, there may be systematic errors which are constant over the entire dimensional range of linewidths; in this case, a straight line fitted to the linewidth differences would have a slope of zero. There may be systematic errors which are not constant, but can be eliminated by a correction based on a calibration curve. The minimal requirements for successfully calibrating a measurement system are the following: (1) the data be in a state of statistical control [22], (2) the data points be scattered in such a way that there are no discernible patterns indicating operator differences or large day-to-day differences in the system, and (3) the data points fall roughly along a smooth curve such as a straight line.

Examples of typical measurement problems encountered in the study are illustrated in figure 5. In figure 5(a) an entire day's measurements are out of control. (See sec. 3.3.2.) In figure $5(\mathrm{~b})$ isolated outliers in the data are so pronounced that the system cannot be successfully calibrated using the linewidth artifact until these outliers are eliminated. (See sec. 3.3.1.) In figure 5(c) there are significant differences between the two operators, and it may be necessary to establish a separate calibration curve for each operator. (See sec. 3.6.3.) In figure $5(d)$ the system is obviously nonlinear; that is, the differences from NBS values do not vary linearly with linewidth, and a calibration curve based on a linear fit may not be adequate to calibrate the system. (See sec.3.6.2.)

+ The raw data from the participants are given in Appendix D, table D-1. 


\subsubsection{Youden Plots}

The individual plots for each measurement system, such as those shown in figure 5, are sufficient to determine measurement differences from NBS, but these plots do not show the relationship of one participant to the other participants. For this reason, plots depicting the linewidth differences from NBS values at each nominal linewidth and each polarity (opaque and clear) for all the measurement systems are given in figures 6 and 7. For these plots, each measurement system is identified by a single letter code. (See table 3.) In cases where more than one difference value is plotted at the same point, a number indicates the total number of coincident values.

Plots of this type are called Youden plots after $\mathrm{W}$. J. Youden who pioneered this method of graphical analysis in the interlaboratory evaluation of test methods. A general discussion of the Youden methodology is given in Appendix $E$ [23]. Basically, two similar objects are measured by each participant, and the measurement of one object is plotted against the measurement of the other object. In the present case, the objects are linewidths of the same polarity and nominal linewidth. For example, the measurements on the nominally 2-jmwide opaque line in row $A$ are plotted against the measurements on the nominally 2-um-wide opaque line in row $C$. However, each company measured a different artifact; therefore, in order to compare all the measurement systems on one Youden plot, the points plotted are the measurement differences from NBS. The origin $(0,0)$ indicates no difference from NBS values. In this type of plot, it is easy to spot the measurement systems that are far removed from the others and also to identify the systems with differences greater than any given amount.

Most of the linewidth differences plotted in figures 6 and 7 are clustered about a 45-deg line passing through the origin, thereby indicating a combination of systematic and random errors. If only systematic differences existed among the systems, all the points would fall on this line; that is, a measurement system that consistently produces results that are high relative to NBS values on both rows $A$ and $C$ by the same amount has only systematic differences. Small departures from the 45-deg line are attributed to random error. Systems which give points distinctly removed from this line are not producing consistent results. Furthermore, when all of the points for the eight repetitions are sufficiently close together and close to the 45-deg line, but removed from the origin, the system is showing consistent systematic error and a small random error. In this case, it should be possible to eliminate the systematic error by a proper calibration procedure.

The Youden plots shown in figures 6 and 7 answer the following question: Are the participants in agreement with each other and with NBS in measuring linewidths on the artifact? The answer to both parts is largely no. In general, there are systematic differences among the participants and between each participant and NBS. This is consistently true for both polarities (opaque and clear) and for all linewidths from 0.5 through $12 \mathrm{~m}$ with the differences being somewhat larger for the larger linewidths. Only six measurement systems showed differences from NBS greater than $0.5 \mu \mathrm{m}$. 


\subsection{Detection of Outliers}

Before proceeding with the analysis, all data on rows $A, B$, and $E$ were edited for outliers. An outlier is an observation that appears to deviate markedly from other members of the sample in which it appears [24]. It is necessary to eliminate such discordant values from the calibration data because even a single outlier can seriously perturb the calibration curve. Two types of outliers are identified in the data as given in Appendix D, table D-1. Isolated outliers are flagged by a zero ( 0 ), and an entire day's measurements that are out of statistical control and considered outliers are flagged by two's (2).

\subsubsection{Isolated Outliers}

The choice of an appropriate test for eliminating outliers is complicated by the variety of data involved. The test that was used is recommended as a good general approach even though the probability levels cannot be computed exactly [25]. This test works well for the interlaboratory data in that it basically rejects only those points which appear to be out of statistical control on the difference plots for each measurement system.

The rejection criterion for individual points is based on the assumption that the measurements $Y_{i j}$ can be described by the following model:

$$
y_{i j}=\gamma+\alpha_{i}+\beta_{j}+\varepsilon_{i j}
$$

$$
\begin{aligned}
& i=1, \ldots, k \\
& j=1, \ldots, m
\end{aligned}
$$

where $k$ is the number of repetitions, $m$ is the number of patterns per row ${ }^{\dagger}, \varepsilon_{i j}$ are random errors, and the $(k+m+1)$ parameters $\gamma, a_{i}$, and $B_{j}$ are unknowns to be estimated.

For each measurement $Y_{i j}$, a residual $\delta_{i j}$ is computed as follows:

$$
\delta_{i j}=y_{i j}-\left(\hat{\gamma}+\hat{\alpha}_{i}+\hat{\beta}_{j}\right)
$$

where the caret $(\wedge)$ signifies the least-squares estimate of the parameter.

The measurement is rejected if $t_{i j}^{*}>3.5$ or if $t_{i j}^{*}<-3.5^{\S}$ where

$$
t_{i j}^{\star}=\frac{\delta_{i j}}{s_{\delta_{i j}}}
$$

and $\mathbf{s} \delta_{i j}$ is the standard deviation of the residual. See Appendix $G$ for a definition of $\mathrm{s}_{\delta_{i j}}$.

+ Since there are ten lines in each row, $m=10$ in most cases. In those cases where data are missing, $m<10$.

$\S$ The distribution of the $t^{*}$ statistic is not known, and therefore an exact probability level cannot be computed for this test. However, the critical value of 3.5 corresponds to the 0.999 probability level for Student's t-test with 60 degrees of freedom. 
This rejection criterion was applied to all the data (rows A, B, C, D, and E) three times iteratively to guarantee that extreme outliers could not mask the effect of other outliers; that is, after an outlier was identified and removed, the test was repeated. Twenty isolated outliers were identified by this test for a total of eight measurement systems. Three of the isolated outliers are shown in figure $5(b)$. (Two other data points were considered to be transcription errors and are also flagged with a zero in Appendix $D$, table table $\mathrm{D}-1$. )

\subsubsection{Entire Day's Measurements as Outliers}

The identification of an entire day's measurements as outliers is based on the assumption that each day's measurements can be described by a linear function of the NBS linewidth values. (See sec. 3.4.) The intent is to test whether or not the data for one day have a different intercept and slope than the data for the other days combined. First, for all measurements $y_{i j}$, the least squares estimates of the
coefficients are obtained for a straight line of the form

$$
y_{i j}=\alpha_{0}+\beta_{0} x_{j} \quad \begin{aligned}
& i=1, \ldots, k \\
& j=1, \ldots, m
\end{aligned}
$$

where $\mathrm{x}_{j}$ are the NBS linewidth values, $\alpha_{0}$ is the intercept, and $\beta_{0}$ is the slope.

Secondly, to test the measurements for day $p, y_{p j}(j=1, \ldots, m)$, the following indicator function $x^{*}$ is defined as follows:

$$
x_{i}^{*}=\left\{\begin{array}{llll}
1 & \text { if } & i=p \\
0 & \text { if } & i \neq p
\end{array}\right.
$$

This function allows for an incremental change in the slope or intercept for day $p$ by fitting all the measurements to an equation of the form

$$
y_{i j}=\alpha_{1}+\alpha_{2} x_{i}^{*}+\beta_{1} x_{j}+\beta_{2} x_{j} x_{i}^{*} \quad \begin{aligned}
& i=1, \ldots, k \\
& j=1, \ldots, m
\end{aligned}
$$

where $\alpha_{1}, \alpha_{2}, B_{1}$, and $\beta_{2}$ are coefficients to be estimated.

The sum of squares for error for eq (1) has n-2 degrees of freedom and is given by

$$
\operatorname{SSE}(1)=\sum_{i=1}^{k} \sum_{j=1}^{m}\left(y_{i j}-\hat{\alpha}_{0}-\hat{\beta}_{0} x_{j}\right)^{2}
$$

where the caret $(\boldsymbol{\wedge})$ signifies a least-squares estimate of the coefficient, and $\mathrm{n}$ is the total number of measurements.

The sum of squares for error for eq (2) has $n-4$ degrees of freedom and is given by

$$
\operatorname{SSE}(2)=\sum_{i=1}^{k} \sum_{j=1}^{m}\left(y_{i j}-\hat{\alpha}_{1}-\hat{\alpha}_{2} x_{i}^{*}-\hat{\beta}_{1} x_{j}-\hat{\beta}_{2} x_{j} x_{i}^{*}\right)^{2} .
$$


An F-test is used to decide if the fit from eq (2) effects a significant reduction in the sum of squares for error. All the measurements for day $p$ are rejected if

$$
F=\frac{(n-4)}{2} \frac{\operatorname{SSE}(1)-\operatorname{SSE}(2)}{\operatorname{SSE}(2)}>F_{.01}\left(\nu_{1}, v_{2}\right)^{+}
$$

where $\nu_{1}$ is the degrees of freedom in the numerator of $F$, and $\nu_{2}$ is the degrees of freedom in the denominator of $\mathrm{F}$.

This F-test was applied to the data from rows A, B, and E for all measurement systems. In all cases for which an out of control condition was identified for either row A (opaque lines) or row B (clear lines) or both, the corresponding measurements in rows C (opaque lines) or row D (clear lines) or both could readily be identified as also out of control by inspecting the difference plots of the type shown in figure 5.

Nine days of measurements from a total of five measurement systems were rejected by this test. This means that one-fourth of the measurement systems experienced problems severe enough to invalidate an entire day's measurements. Thus, problems of this type should not be regarded as rare events, but are to be expected in day-to-day operations.

\subsection{Calibration Curves}

The derivation and application of calibration curves in this study involves the following steps for each measurement system: (1) a linear line-spacing calibration curve is derived from measurements on row $\mathrm{E}_{\text {; }}$ (2) a statistical test is used to determine the significance of the slope and intercept of the line-spacing calibration curve, and if either the intercept or slope, or both, are found to be statistically significant, the line-spacing calibration is used to correct linewidth measurements from rows $A, B, C$, and $D$; (3) a linear linewidth calibration curve is derived from measurements of opaque lines on row $A$ where these linewidth values may have been corrected for line spacing in step 2; (4) a statistical test, similar to that in step 2, is used to determine the significance of the slope and intercept of the linewidth calibration curve, and if either the intercept or slope, or both, are found to be statistically significant, the linewidth calibration is used to correct linewidth measurements from row $C$; and (5) steps 3 and 4 are repeated for clear lines on rows $B$ and $D$, respectively.

The linear calibration curves are derived from a least-squares technique [26] that treats the participant's repeated measurements of each pattern as the unknown variable and the corresponding NBS value as the known variable. $\$$

T The critical value $\mathrm{F}_{.01}\left(v_{1}, v_{2}\right)$ is obtained from a table of the $\mathrm{F}$ distribution for a probability level of 0.99 with $v_{1}=2$ and $v_{2}=(n-4)$.

$\S$ In an unpublished work, Bruce Hoadley, Statistical Engineering Division, NBS, shows that the linear fit is not significantly affected by both variables being in error if the standard deviation of the known variable is a sufficiently small fraction of the intervals between the known values. In this case, the standard deviation in the NBS linewidth values could be as large as $0.35 \mu \mathrm{m}$ without affecting the results. 
This technique guarantees that the standard deviations of the linear fits are proper estimates of the measurement process standard deviation. The general form of this calibration curve is discussed in Appendix F.

The assumption has been made that a linear calibration curve is adequate to describe the relationship between the participant's measurements and the NBS values. An examination of the plots of differences between the participant's measurements and NBS values suggests that this assumption is satisfactory for most measurement systems. (For example, see figs. 10 and 11.) The linearity assumption is discussed further in section 3.6.2.

\subsubsection{Line-Spacing Calibration}

We now address the following question: Is a correction based solely on a line-spacing calibration ( $r o w \mathrm{E}$ ) sufficient to bring the participants into agreement among themselves and with NBS? The line-spacing calibration curve is described by the following model:

$$
y_{i j}=\alpha+\beta x_{j} \quad \begin{aligned}
& i=1, \ldots, w_{j} \\
& j=1, \ldots, m
\end{aligned}
$$

where $y_{i j}$ is the line-spacing measurement, $x_{j}$ is the NBS line-spacing value, $i$ denotes the $i$ th repetition on the same pattern, $j$ denotes the $j$ th pattern in the row, ${ }^{\dagger} w_{j}$ is the number of repetitions of the $j$ th pattern, $\S \alpha$ is the intercept, and $\beta$ is the slope. The least-squares estimates $\hat{\alpha}$ and $\hat{\beta}$ are defined in Appendix $F$ by eqs $(F-5)$ and $(F-6)$, respectively.

Line-spacing calibration curves of the form given by eq (3) were derived for each system from the participant's measurements of the ten patterns in row $E$. The least-squares estimates of the intercept and slope of these calibration curves are given in table 4. If $\alpha=0.0$ and $\beta=1.0$, then no line-spacing correction is needed. Table 4 shows, however, that for all systems the estimated intercepts and slopes are at least slightly different from zero and one, respectively. In order to determine whether or not these differences are statistically significant, and thus whether or not a line-spacing correction should be applied, the following statistics [27] are used in separate tests:

and

$$
t_{1}=\hat{\alpha} / s_{\alpha}
$$

$$
t_{2}=(1-\hat{B}) / s_{B}
$$

where $s_{\alpha}$ and $s_{\beta}$ are the standard deviations of $\hat{\alpha}$ and $\hat{\beta}$, respectively. These standard deviations are given by eqs $(F-7)$ and $(F-8)$ in Appendix $F$.

$\bar{\dagger}=1$ corresponds to the first, or leftmost, pattern in the row; for example, line A-O in row A. (See fig. 3.)

$\S$ Since the data were edited for outliers, $w_{j}<k$ for some measurement systems. 
The intercept is judged significantly different from zero if

$$
t_{1}>t_{.025}(v) \text { or } t_{1}<-t_{.025}(v) \text {, }
$$

and the slope is judged significantly different from one if

$$
t_{2}>t_{.025}(v) \text { or } t_{2}<-t_{.025}(v) \text {, }
$$

where $\nu$ is the degrees of freedom and $t .025(v)$ is the critical value of Student's $t$ distribution with $v$ degrees of freedom that is exceeded with 2.5 percent probability.

As shown in eq (4), the magnitude of the intercept and slope are considered relative to the measurement system precision. Therefore, a value of $\alpha$ far from zero or a value of $\beta$ far from one may not be significant according to the $t$ statistic if the precision is poor (i.e., a large imprecision). In this case, the intercept or slope are not known well enough to provide a reliable calibration curve. On the other hand, a value of a close to zero or a value of $\beta$ close to one may be significant if the precision is good (i.e., a small imprecision). In this case, the calibration curve would be useful for correcting measurements.

A significant finding for the slope implies that the errors vary with the line-spacing dimension, whereas a significant finding only for the intercept implies that there is a constant offset independent of spacing.

The test statistics $t_{1}$ and $t_{2}$ are shown in table 4 for all twenty measurement systems. This table shows that either the intercept or the slope, or both, are significant at the 95 percent probability level for seven measurement systems. Only one of these systems exhibits a relatively constant offset (system 14C). Therefore, a line-spacing correction was applied to the linewidth data on rows $A, B, C$, and D for each of these seven systems as follows:

$$
y_{i j}^{\star}=\frac{y_{i j}-\hat{\alpha}}{\hat{\beta}}
$$$$
\begin{aligned}
& i=1, \ldots, w_{j} \\
& j=1, \ldots, m^{2}
\end{aligned}
$$

where $y_{i j}^{*}$ is the linewidth measurement corrected for line-spacing calibration, $y_{i j}$ is the uncorrected linewidth measurement, and $\hat{\alpha}$ and $\hat{\beta}$ are the least-squares estimates of the intercept and slope of the linear line-spacing calibration curve. For the thirteen measurement systems which do not require line-spacing correction, $y_{i j}^{*}=y_{i j}$.

To assess the effects of the line-spacing correction, the differences between the participant's linewidth measurements corrected for line-spacing and NBS linewidth values were plotted for rows $A$ and $B$ for each of the seven measurement systems identified in table 4 as needing line-spacing correction. Analysis of these difference plots show that the effect of this correction differs widely among the seven systems. For systems $5 \mathrm{~V}$ and $15 \mathrm{~B}$, which show the largest line-spacing differences from NBS values on row $E$, linewidth differences are reduced significantly by the line-spacing correction. For the 
other five systems, there is either a slight reduction or slight increase in the linewidth differences. In no cases are the systematic linewidth differences between the participant's measurements and NBS values eliminated by applying a line-spacing correction.

\subsubsection{Linewidth Calibration}

The next consideration is the following question: If a line-spacing calibration is not sufficient for eliminating all systematic errors, can a linewidth calibration that uses ten lines on the artifact substantially reduce these systematic errors? The linewidth calibration curve for row A or B is described by the following model:

$$
y_{i j}^{*}=a+b x_{j}
$$$$
\begin{aligned}
& i=1, \ldots, w \\
& j=1, \ldots, m^{j}
\end{aligned}
$$

where $y_{i j}^{*}$ is the linewidth measurement corrected for line-spacing calibration, $x_{j}$ is the NBS linewidth value, $a \cdot$ is the intercept, $b$ is the slope, and the subscripts are the same as those defined for eq ( 3 ). The least-squares estimates of $a$ and $b$ are given by eqs $(F-5)$ and $(F-6)$ in Appendix $F$ where a replaces $\alpha, b$ replaces $\beta$, and $y^{*}$ replaces $y$.

Student's t-tests for the linewidth calibration curves similar to the tests for the line-spacing calibration curves in section 3.4 .1 were made. The resulting $t$ statistics and the least-squares estimates of the intercepts and slopes of the calibration curves for both opaque and clear lines are shown in table 5. As indicated in this table, either the intercept of the linewidth calibration curve is significantly different from zero or the slope is significantly different from one, or both, for most of the twenty measurement systems.

For the measurement systems with a linewidth calibration curve that has a slope significantly different from one, the user will benefit from applying this calibration curve to linewidth measurements. For the other measurement systems, the systematic differences from NBS values do not vary significantly over the dimensional range of linewidths, and a correction for a constant offset is probably sufficient.

Since thirty-four out of the forty calibration curves show a statistically significant departure from the ideal intercept $(a=0.0)$ or slope $(b=1.0)$ or both, corrections based on the linewidth calibration curves were applied to the data for all twenty systems in order to simplify the computations. Specifically, a linewidth correction based on row A (opaque lines) was applied to the linewidth data on row $\mathrm{C}$ (opaque lines), and a linewidth correction based on row B (clear lines) was applied to the linewidth data on row $D$ (clear lines) as follows:

$$
y_{i j}^{* *}=\frac{y_{i j}^{*}-\hat{a}}{\hat{b}}
$$$$
\begin{aligned}
& i=1, \ldots, w \\
& j=1, \ldots, m^{j}
\end{aligned}
$$

where $y_{i j}^{* *}$ is the linewidth measurement corrected for both line-spacing and linewidth calibrations, $y_{i j}^{*}$ is the linewidth measurement corrected 
for line-spacing calibration, and $\hat{a}$ and $\hat{b}$ are the least-squares estimates of the intercept and slope of the linear linewidth calibration curve. (For systems not requiring a line-spacing correction, $y_{i j}^{*}=y_{i j} \cdot$ ) The corrected linewidths $Y_{i j}^{*}$ for rows $C$ and $D$ are given in Appendix $D$ table $D-2$.

\subsection{Assessment of Line-Spacing and Linewidth Calibrations}

As discussed in section 3.4.2, the linewidth calibration curves for opaque and clear lines are based on measurements of lines in rows $A$ and $B$, respectively, and measurements of lines in rows $C$ and $D$ are, in turn, corrected by the appropriate calibration curve. For some systems, the linewidth measurements are also corrected for a line-spacing calibration. The following thesis is proposed: if these calibration curves are capable of eliminating systematic errors between the user's system and the NBS system, the corrected linewidth measurements for either opaque or clear lines will have zero offset from the NBS system except for random error.

\subsubsection{Measurement Systems Combined}

In order to assess the effects of both line-spacing and linewidth calibrations on the measurement systems, Youden plots similar to those discussed in section 3.2 .2 are given in figure 8 .

Recall that the points on a Youden plot correspond to measurements on two similar objects plotted against each other and that the pattern created by the accumulated points over all repetitions indicates the nature of the errors affecting the measurement systems. In the case of uncorrected linewidth measurement differences from NBS as shown in the Youden plots of figures 6 and 7, the errors are a combination of systematic and random errors as indicated by the points clustering about a 45-deg line.

It seems reasonable to use the corrected measurements on the $C$ and $D$ rows as the two similar objects in a Youden plot in which the corrected linewidth measurement differences from NBS are plotted against each other for lines of the same nominal size. The proposed thesis is demonstrated if the resulting points form a circle about the origin $(0,0)$, thereby indicating only random errors. If the center of the circle is substantially offset from the origin or if the points cluster along a line, the calibration curves are not adequately correcting for one or both line polarities.

Figure 8 shows the Youden plots of the differences between the corrected linewidth measurements and NBS values for opaque lines (row C) and clear lines (row D). ${ }^{\dagger}$ A comparison of figure 8 with figures 6 and 7 shows that the points for the corrected measurements are distributed relatively evenly among the four quadrants of a circle centered at or near the origin. Therefore, most of the systematic linewidth error has been removed for the measurement systems by using linewidth calibration curves.

T When an entire day's measurements have been identified as outliers for rows $A$ or $B$, the corresponding day's measurements for rows $C$ or $D$ have been deleted from figure 8 because the calibration curve is not capable of rectifying out-of-control conditions. (See sec. 3.3.2 and Appendix D.) 


\subsubsection{Individual Measurement Systems}

For each measurement system, a comparison of the raw data on rows $C$ and $D$ is made with the same data corrected for both line-spacing (if required) and linewidth calibrations. Frequency tables of differences from NBS values for both the raw data (R) and the corrected data (C) are given in table 6 for row $\mathrm{C}$ and in table 7 for row D. The frequencies for the raw and corrected data are given in the left and right columns, respectively, for each measurement system. These frequency tables show the changes in the distribution of linewidth-measurement differences from NBS brought about by the line-spacing and linewidth calibrations.

The linewidth calibration should produce a symmetric distribution of corrected linewidth values that are centered about a zero difference and approximate a normal distribution. For a few systems, the raw data already shows such a symmetric distribution. For almost all the other systems, however, there is a definite improvement with the linewidth calibration. In the few cases where the improvement is not sufficient, the between-day or between-operator measurement differences may be too large or a linear calibration curve may be inadequate. (See sec. 3.6.)

\subsubsection{Measurement Process Standard Deviation}

For this study, estimates of the process standard deviation for linewidth measurements corrected for line spacing were made for each measurement system. This standard deviation is given by

$$
\left[\frac{\sum_{j=1}^{m} \sum_{i=1}^{w}\left(y_{i j}^{*}-y_{j}^{\prime}\right)^{2}}{(n-2)}\right]^{1 / 2}
$$

where

$$
n=\sum_{j=1}^{m} w_{j}
$$

and $y_{j}^{\prime}=\hat{a}+\hat{b x}_{j} \cdot$ values of $s_{L}$ are shown in table 8 for both opaque and clear lines for all twenty measurement systems.

\subsubsection{Uncertainty of Corrected Linewidth Values}

A crucial factor in assessing the possible benefits derived from calibrating an optical linewidth-measurement system by using an NBS calibrated linewidth artifact is the resulting measurement uncertainty. This uncertainty includes the limits to random error for the measurement system and any systematic errors affecting the measurement process. The uncertainty associated with the NBS values assigned to the artifact is a systematic error in the participant's measurement process.

The limits to random error for the measurement system must take into account not only the variability in measuring the individual linewidths, but also the 
error in deriving the linewidth calibration curve which is used to correct the linewidth measurements. The calibration curve has an inherent uncertainty because if it were to be rederived at another time it may not duplicate the initial calibration curve. Therefore, the data for deriving the calibration curve are a determining factor in the measurement process uncertainty. The problem of combining these errors is difficult [28] and is addressed in reference 29 , the results of which have been applied to this analysis.

Table 8 gives limits to the random error $E$ for a single measurement of either an opaque or clear line. The interpretation of $\mathrm{E}$ is that the probability is greater than 0.99 that 95 percent of the intervals $y * * \pm E$ cover the NBS linewidth value where $y^{* *}$ is the corrected linewidth value. (See sec. 3.4.2.) The total uncertainty for the measurement process is

$$
v= \pm\{E+\mid \Delta \text { NBS } \mid\}
$$

where $\Delta_{\text {NBS }}$ is the uncertainty for the NBS linewidth values. The NBS measurement uncertainties are listed in table 1.

Table 8 shows that about half of the measurement systems in this study have random error limits $(E)$ that are greater than $0.25 \mu \mathrm{m}$. Thus, the uncertainty intervals for the corrected linewidth values are at least $0.5 \mathrm{\mu m}$. Adding the NBS measurement uncertainties further increases these intervals. (See sec. 2.2.) These uncertainty intervals should not, however, be considered representative of current measurement processes with these systems since the measurement data were edited for outliers before deriving the calibration curves. Instead, these uncertainties represent possibly the best values that a user could achieve with an effective measurement control program.

\subsection{Sources of Measurement Errors}

It is difficult to pinpoint the cause of the relatively large measurement uncertainties associated with most of the measurement systems. (See table 8.) The magnitude of the process standard deviation $\mathrm{s}_{\mathrm{L}}$ shown in table 8 is obviously important. Other possible sources of error are: (1) inability to repeat the calibration curve from day to day, (2) inadequacy of the linearity assumption for the calibration curve, and (3) large differences among different operators. The interlaboratory study was designed to reveal the presence of these types of errors which are addressed in the following discussion.

\subsubsection{Between-Day Differences in Calibration Curves}

In order to use a calibration curve based on several days' measurements, the same operator should be able to repeat the calibration curve with the same measurement system; i.e., the system must be in a state of statistical control. In the present case, the slope and intercept of the linewidth calibration curve must remain constant throughout all sets of measurements made by the same operator.

Figure 9 shows two examples of linewidth calibration curves that change daily for one operator on a given measurement system. (The data in figure $9(a)$ are 
for a different operator and measurement system than the data in figure 9(b).) The linewidth measurement differences for one day are connected by solid lines, and the corresponding linewidth calibration curve is represented by a dashed line. In figure $9(a)$ the slope of the calibration curve is changing from day to day while the intercept is relatively fixed. In figure 9 (b) both the intercept and the slope are changing from day to day. Obviously, the calibration curve for both of these examples does not repeat satisfactorily from day to day, and the operator or measurement system or both are out of statistical control.

A test statistic has been devised to determine if the slope and intercept of the linewidth calibration curve remain the same for each day's measurements. For measurements $y_{i j}$ on a given day, this test statistic is based on the following linear model:

$$
y_{i j}=\alpha_{i}+\beta_{i}\left(x_{j}-x\right)
$$

$$
\begin{aligned}
& i=1, \ldots, k \\
& j=1, \ldots, m
\end{aligned}
$$

where

$$
x=\frac{\sum_{j=1}^{m} x_{j}}{m}
$$

$x_{j}$ is the NBS linewidth value, $i$ denotes the day or $i$ th repetition, $j$ denotes the $j$ th line in the row, and $\alpha_{i}$ and $\beta_{i}$ are the intercept and slope, respectively, for each repetition. The least-squares estimates $\hat{\alpha}_{j}$ and $\hat{\beta}_{i}$ for the $i$ th repetition are given by eqs $(F-5)$ and $(F-6)$ in Appendix $F$ where $x_{j}-x_{0}$ replaces $x_{j}$.

The test statistic is given by

where

$$
F=\frac{1}{2(k-1)}\left[\frac{\sum_{i=1}^{k}\left(\hat{\alpha}_{i}-\hat{\alpha}\right)^{2}}{\frac{1}{k} \sum_{i=1}^{k} s_{i}^{2}}+\frac{\sum_{i=1}^{k}\left(\hat{\beta}_{i}-\hat{\beta}^{2}\right)^{2}}{\frac{1}{k} \sum_{i=1}^{k} s_{i}^{2}}\right]
$$

$$
\hat{\alpha}_{.}=\frac{\sum_{i=1}^{k} \hat{\alpha}_{i}}{k} \text { and } \hat{\beta}_{.}=\frac{\sum_{i=1}^{k} \hat{\beta}_{i}}{k} \text {. }
$$

If $F>F_{.01}[2(k-1), k(m-2)]$, the calibration curve is not satisfactorily repea ted from day to day.

The $F$ statistics and the pooled standard deviations $s_{P}$ resulting from fitting a separate calibration curve to each day's linewidth measurements are 
listed in table $9^{\dagger}$. There is a separate analysis for each operator on rows $A, B, C$, and D. All the data were used, including values previously identified as outliers. Even when the standard deviations shown in table 9 are consistent, the slope or intercept of the calibration curve or both can change significantly.

The test statistic is fairly conservative; that is, it flags only large day-to-day changes in the calibration curve. A system that has several asterisks in table 9 has a serious day-to-day control problem.

There is also ample evidence from this analysis that the two operators should, for the most part, be treated separately. As indicated in table 9, there are control problems with one or both operators for all measurement systems except system $3 \mathrm{~V}$ and $11 \mathrm{~F}$. For several of the systems, such as $1 \mathrm{I}$ and 5U, there are also control problems associated with only one line polarity (clear or opaque). From this analysis users can hopefully recognize day-today variations in their measurement systems and possibly identify the sources of this variability.

\subsubsection{Linearity Assumption}

A test based on the measurement repetitions was used to decide if a linear fit is appropriate for a particular calibration curve [30]. In this test, the computed values at each linewidth as given by

$$
y_{j}^{\prime}=\hat{a}+\hat{b} x_{j}
$$

are compared to the average of the repetitions at each linewidth, namely

$$
y^{*}{ }_{\cdot j}=\frac{\sum_{i=1}^{w_{j}} y_{i j}^{*}}{w_{j}}
$$$$
j=1, \ldots, m
$$

where $w_{j}$ is the number of repetitions on the $j$ th line.

Close agreement between the hypothesized curve and these averages is evidence that the hypothesis is correct. The test is based on an F statistic, and the assumption of linearity is rejected if

$$
F=\frac{1}{m-2} \cdot \frac{(n-2) s_{L}^{2}-(n-m) s_{R}^{2}}{s_{R}^{2}}>F_{.01}(m-2, n-m)
$$

where $\mathrm{n}$ is the total number of measurements, $m$ is the number of measurements per row, $S_{L}$ is the measurement process standard deviation as given in sec. 3.5.3, and $s_{R}$ is the standard deviation of the repetitions given by

T To compute these pooled standard deviations, the separate standard deviations for each repetition were combined for each operator. 


$$
S_{R}=\left[\frac{\sum_{j=1}^{m} w_{j}\left(y_{j}^{*}-y^{*}{ }^{*}\right)^{2}}{n-m}\right]^{1 / 2}
$$

This analysis has been applied to the linewidth data on rows A and B corrected for line-spacing calibration. The resulting standard deviations of the repetitions $\mathrm{s}_{\mathrm{R}^{\prime}}$ process standard deviations $\mathrm{s}_{\mathrm{L}^{\prime}}$, degrees of freedom $V$, and $F$ statistics are given in table 10. As shown in this table, only seven of the forty calibration curves have $F$ values that are significant. significant $F$ value implies that the system response is non-linear. Poor system precision may mask non-linear behavior for some systems, and a significant $F$ value for a system with very good precision, for example system $3 \mathrm{~V}$, would not necessarily mean that the calibration technique is inappropriate but only that it may be improved upon. Considering the level of system precision shown in table 8 , non-linearity of the linewidth calibration curve is not a significant source-of measurement error for the majority of systems used in this study. In general, an interpretation of the failure of the linear calibration model is best made by examining the difference plots, such as those shown in figure 5, to determine whether a higher-order calibration curve is indicated or whether only certain regions of the curve are non-linear.

\subsubsection{Differences Between Operators}

As discussed in section 3.6.1, two operators on the same measurement system often show significant measurement differences. Some of the difference plots for the individual measurement systems also show these operator differences. [For example, see fig. 5(c).] These operator differences were verified by an analysis of variance [31]. Such operator differences raise the question of whether or not it would be beneficial to have each operator maintain his or her own calibration curve.

To help answer this question, the two operator/system combinations for each of three measurement systems (1I, 8I, and 8P) that showed the largest differences between operators were analyzed as separate measurement systems. Linewidth calibration curves based on measurements from rows $A$ and $B$ were derived for each of these six operator/system combinations, and the linewidth measurements in rows $\mathrm{C}$ and $\mathrm{D}$ were corrected based on the appropriate calibration curve. The results are shown in table 11.

Table 11(a) shows the frequencies of differences from NBS for the raw and corrected linewidth values on row $\mathrm{C}$ for the six operator/system combinations. Table 11(b) shows similar data for row D. In order to assess the results of using separate calibration curves for each operator as compared with using a combined calibration curve for both operators, compare the corrected frequencies in table 11(a) with table 6 and the corrected frequencies in table 11(b) with table 7. The interval from -0.05 up to $0.05 \mathrm{~mm}$ is of particular interest. Systems 8I and 8P show considerable improvement for the clear lines, and system 1I shows considerable improvement for the opaque lines. For the other combinations of system and polarity, the improvement is only slight thereby indicating that we may not have accounted for all the sources of 
error by using separate calibration curves. The overall conclusion is that separate calibration curves for each operator may result in significant differences between corrected linewidth measurements made on the same system.

\subsection{Effects of Using Adjusted NBS Linewidth Values}

As shown in table 1, there are systematic differences of about 0.1 um between the original linewidth measurements on the artifacts and their measurements on an improved version of the NBS scanning optical microscope system. For this reason, an effort was made to determine the effects on the data analysis of using NBS linewidth values that were adjusted to account for these systematic differences.

For the video image-scanning measurement systems, participants used the original NBS-furnished line-to-space ratio (pattern $F-5$ ) to set the electronic edge-detection threshold; thus, it was not possible to determine the effects of using adjusted NBS values. For the other measurement systems, however, it was found that these effects were small. For example, the magnitude of the linewidth differences between the participant's measurements and NBS values changed slightly for some systems resulting in a slightly different calibration curve. However, such minor changes do not significantly affect the data analysis presented in the preceding sections nor do they change the basic discussion and conclusions presented in the following sections. 


\section{DISCUSSION}

The interlaboratory study and the statistical analysis of the resulting data were designed to answer several questions about the artifact and measurement procedures developed by NBS, as well as questions about the measurement systems used by the participants. The previous section on statistical analysis addresses some of these questions. The present section elaborates on these and related questions, provides guidelines to the participants for interpreting their results, and discusses the implications of these results for the development of the NBS linewidth standard, Standard Reference Material (SRM) 474.

\section{1 NBS Recommended Procedures}

The recommended procedures followed by the participants in the study were significantly different from most of the procedures used in the IC industry for linewidth measurements on AR-chromium photomasks. The procedures used in industry typically differ between companies, between divisions within a company, and sometimes even between operators within the same group in a company. For example, the illumination systems in optical-microscope measurement systems are usually not adjusted according to one accepted method, such as Kohler illumination, and no effort is made to control the degree of coherence in the illumination system. As a result, there arise a large number of different optical line-image profiles on which linewidth measurements are made [32].

In the present study, the recommended procedures were designed (1) to eliminate measurement errors which may have been introduced if the participants had adjusted their measurement systems in accordance with their own procedures and standards; (2) to reduce certain known sources of errors, such as chromatic aberrations in the optics and mechanical backlash in micrometer attachments; and (3) to provide an optical line image with steep edge gradients, thereby reducing the effects of image-contrast variation among different measurement systems and improving the repeatability of edge settings. The basic intent of these procedures was to provide a similar optical line image with steep edge gradients in all measurement systems used in the study so that any linewidth measurement differences among these systems would be dependent primarily on the different edge-detection criteria and differences in repeatability.

The differences between the procedures used in this study and the many techniques used in industry for linewidth measurements must be considered in making comparisons of the participant's measurements with NBS values as well as in determining the degree of improvement in linewidth measurements that each participant can expect from using an NBS linewidth standard and recommended measurement procedures. Therefore, the present discussion applies primarily to this interlaboratory study and the eventual use of the NBS linewidth standard (SRM 474) and measurement procedures which are based on those used in the study.

For the most part, the participants reported little difficulty in following the recommended measurement procedures. In a few cases, the participants 
suggested changes and, whenever possible, these changes and other changes based on recent NBS work have been made to the procedures.

\subsection{Linewidth Calibration Curves}

The Youden plots given in figures 6 and 7 clearly show the presence of systematic errors in the participants' raw linewidth measurements. Although the differences from NBS values are, in a few cases, as large as 1 um, more than 80 percent of the raw data exhibit systematic errors equal to or less than $\pm 0.3 \mathrm{um}$ for both opaque and clear lines. Other studies, in which linewidth measurements were made under conditions more closely representing those found in industry, show significantly larger measurement differences from NBS [18, 33]. Thus, it appears that even before a linewidth calibration is applied to the data, the procedures and artifacts used in the present study reduce measurement differences among different systems.

Only seven of the twenty measurement systems show significant differences between line-spacing measurements and NBS values. Thus, the single linespacing value furnished by NBS to the participants was, in most cases, sufficient for generating the proper length scale over the $0.5-$ to $12-\mu \mathrm{m}$ range. However, the line-spacing correction which was applied to the seven systems showing significant line-spacing differences from NBS does not eliminate all the systematic errors in the linewidth measurements. It is not surprising that a proper line-scale adjustment to the measurement system or a line-spacing correction to the data does not eliminate all the systematic error in a linewidth measurement. As discussed in reference 16, a linewidth measurement, in contrast to a line-spacing measurement, involves systematic errors of opposite signs in locating each edge of the line, and these errors do not cancel as do the systematic errors of equal sign and magnitude in a line-spacing measurement.

The application of a linewidth calibration curve largely reduces the systematic errors remaining in the participant's data after correction for line-spacing differences from NBS. In a few cases, the linewidth calibration curve is not very effective because the raw data show systematic and random errors due to operator differences, day-to-day differences, or the inadequacy of a linear calibration curve. For a few systems, the linewidth calibration curve is largely unnecessary because the system was initially adjusted to make it agree with both the NBS-furnished line-spacing value and the line-tospace ratio. For these measurement systems, however, the derived linewidth calibration curves still show a significant slope; thus, a single NBSfurnished line-to-space value is not entirely adequate for adjusting these systems to measure linewidths over the entire $0.5-$ to $12-\mu \mathrm{m}$ range.

\subsection{Polarity Dependence of Linewidth Calibration}

For most of the measurement systems, the systematic errors for measurements of opaque lines in rows $A$ and $C$ are opposite in sign to the systematic errors for measurements of clear lines in rows $B$ and $D$. An example of this linepolarity dependence is shown in figure 10. The polarity dependence varies with the type of measurement system and, in some cases, with the operator. 
In all cases, the polarity dependence arises from using an incorrect optical threshold to locate the edge [34]. In the ideal case of identical, but inverted, line-image profiles for opaque and clear lines, the systematic errors resulting from using an incorrect optical threshold would be opposite in sign and equal in magnitude. Although some of the measurement systems show systematic errors for rows $A$ and $C$ that are approximately equal to those for rows $B$ and $D$ but of opposite sign, many of the systems also show systematic errors that are different in both magnitude and sign. (See fig. 10.) One reason for this behavior may be the effects of flare or scattered light in the measurement system. Opaque lines on a clear background (row A) may introduce more flare, which alters the line-image profile, than clear lines on an opaque background (row B). As a result, the line-image profiles for opaque and clear lines would no longer be identical (except for inversion). Thus, using an incorrect optical threshold on these profiles would give different systematic errors for linewidth measurements of opaque and clear lines.

The two systems for which a linewidth calibration curve is largely unnecessary still show some polarity dependence for the relatively small residual differences from NBS. These systems were initially adjusted by the participants so that the line-to-space ratio in pattern F-5 equaled the NBS value. (See sec. 2.3.) This adjustment eliminated most of the systematic error for row $A$ at a linewidth of $5 \mathrm{um}$ which was the nominal width of both the opaque and clear lines in pattern F-5. As shown in figure 11(a) for one of these two systems, the average difference at $5 \mu \mathrm{m}$ for the five data sets on row $A$ is close to zero. However, figure $11(b)$ shows the average difference at $5 \mu \mathrm{m}$ on row B for this system to be close to $0.05 \mu \mathrm{m}$. Again, the effects of flare light may be responsible for this behavior. Pattern F-5 resembles the opaque lines on a clear background in row A more closely than it resembles the clear lines on an opaque background in row B. Therefore, adjusting the system to pattern F-5 would largely take into account the flare present in the image profile for an opaque line in row A, but would not correct the system for the different amount of flare present with a clear line in row $B$.

\subsection{Measurement Process Standard Deviation}

The estimates of the standard deviation of the measurement process, as given in table 8, are close to or greater than $\pm 0.1 \mathrm{~m}$ for about half of the measurement systems. These relatively large values indicate that although operators measured an artifact with lines that have steep material-edge profiles and used procedures to provide an optical line image with steep edge gradients, there is still difficulty with some systems in repeating line-edge settings. Because the standard deviations in table 8 are based on measurement data that exclude outliers (see sec. 3.3), these standard deviations, in spite of their large values, are not necessarily representative of the participant's measurement process but are representative of a measurement process that is in some degree of statistical control.

The F statistics in table 9 show that some systems have considerable difficulty maintaining a calibration curve with constant intercept and slope. In order to utilize effectively a calibration technique, the operator must be able to repeat the calibraticn curve for any measurenent sequence; in this 
case, the resulting standard deviation would be an estimate of the measurement process precision. Where the calibration curve cannot be successfully repeated from day to day, the measurement process is usually said to have a between-day component of variance. In this situation, it is not possible to reduce substantially the size of the uncertainty of a reported linewidth value by taking several measurements of the linewidth over a short period and using the average value. The measurements would have to be spread out over the same base period used for deriving the calibration curves.

In cases where there are significant differences between operators, calibration curves based on only one operator are not applicable for correcting measurements made by the other operator. Calibration curves based on pooling the data for both operators in this case would give a relatively large standard deviation and uncertainty associated with correcting a linewidth measurement.

\subsection{Linearity of Calibration Curve}

Table 10 shows that linear calibration curves are adequate for most of the measurement systems. For these systems, the artifact has a sufficient number of linewidths over the $0.5-$ to $12-\mu \mathrm{m}$ range to derive a linear fit. The systems requiring a nonlinear calibration curve show nonlinear behavior over the lower or upper end of the dimensional range. [For example, see fig. $5(b)$.$] Nonlinear behavior at the lower end of the dimensional range where$ the linewidth is approaching the limiting resolution of the microscope optics is expected [35]. Most of the optical systems used in the present study did resolve the smaller lines. The nonlinear behavior at the upper end of the dimensional range may be due to optical distortion in the outer part of the field of view of the optical microscope. The edges of the larger lines are located in the outer part of the field of view when they are centered for a measurement, and if distortion is present, these edges would appear curved. 


\section{CONCLUDING REMARKS}

The present interlaboratory study has provided considerable information on the usefulness of the NBS linewidth-measurement artifact and the associated procedures for measuring linewidths on photomasks in transmitted light. In addition, the study has shown the effects of using this artifact and procedures to calibrate a number of different types of measurement systems commonly used by the IC industry to measure linewidths on photomasks. These results are discussed in detail in previous sections of this report and are summarized below.

5.1 The participants exhibit systematic differences among themselves and from NBS on linewidth measurements of clear and opaque lines. These differences are, in a few cases, as large as $1 \mu \mathrm{m}$, but more than 80 percent of the raw data shows differences less than $\pm 0.3 \mathrm{um}$. Although a line-spacing calibration improves some of the largest systematic errors, this type of calibration is not sufficient to bring the participants into agreement with NBS.

5.2 A linear form of the linewidth calibration curve is sufficient for most of the measurement systems used in the study. In some cases, a constant offset may be sufficient over the dimensional range of 0.5 to $12 \mathrm{\mu m}$. For some systems, the initial adjustment of the system in accordance with an NBSfurnished line-to-space ratio and a line-spacing value may be sufficient for calibration.

5.3 A separate calibration curve or constant offset is required for measuring lines of different polarity (opaque and clear). Many of the measurement systems appear to have flare, or scattered light, which affects the magnitude of the systematic error for opaque lines as compared with the systematic errors for clear lines.

5.4 The data from this study clarify the need for measurement-control procedures in many of the measurement systems. Single outliers in the data are frequent. For several systems, an entire set of measurements (taken during a single day by a single operator) is out of statistical control.

5.5 The standard deviation of the measurement process is close to or greater than $\pm 0.1 \mu \mathrm{m}$ for about half of the measurement systems. For some systems, there are also significant day-to-day differences and operator differences. In these cases, the calibration curve may not be applicable to measurements made on any given day, and calibration curves based on one operator may be restricted to measurements by the same operator.

5.6 The linewidth-measurement data from the operator/measurement systems used in the study fall into one of the three following categories: data with repeatability errors so large that a correction for systematic errors is not meaningful for a single linewidth measurement; (2) data with repeatability errors sufficiently small that corrections for systematic errors can be made using a calibration curve for the measurement system based on a reasonably small number of repeated measurements; and (3) data with insignificant repeatability errors such that small systematic errors can be corrected by instrument adjustment, a calibration curve, or a constant offset 
from NBS values over the entire dimensional range. Repeatability refers to the variability between replicate measurements made on different days with one operator/measurement system combination.

5.7 The NBS artifact and the recommended procedures are basically adequate for calibrating an optical-microscope linewidth measurement system in the 0.5 - to $12-\mu \mathrm{m}$ region provided that the system is in a state of statistical control. Based on the results of this study, the pattern on the artifact was changed slightly and the procedures were rewritten to improve their usefulness. The improved version of the artifact is being made available as SRM 474 (Optical Microscope Linewidth Measurement Standard). The new measurement procedures are being written as standard practices and test methods in cooperation with the American Society for Testing and Materials (ASTM). In addition, the statistical procedures used in this study for deriving and applying a calibration curve to reduce systematic measurement errors, as well as statistical procedures for determining if the measurement system is in a state of statistical control, are presented in NBS Technical Note 1164 [36]. 


\section{REFERENCES}

1. Nyyssonen, D., and Jerke, J. M., Optical Linewidth Measurement - A Basic Understanding, Proc. Microelectronics Measurement Technology Seminar, San Jose, California, February 6-7, 1979, pp. 251-266.

2. Jerke, J. M., ed., Semiconductor Mea surement Technology: Accurate Linewidth Measurements on Integrated-Circuit Photomasks, NBS Spec. Publ. 400-43, pp. 78-94 (February 1980).

3. Swyt, D. A., Rosberry, F. W., and Nyyssonen, D., Calibration of Optical Microscopes for Photomask Linewidth Measurements, Proc. Microelectronics Seminar INTERFACE '77, Monterey, California, October 5-7, 1977, Publication No. G-48, pp. 131-144 (Eastman Kodak Co., Rochester, New York, 1978).

4. Swyt, D. A., An NBS Physical Standard for the Calibration of Photomask Linewidth Measuring Systems, Effective Utilization of Optics in Quality Assurance 129, pp. 98-105 (Society of Photo-Optical Instrumentation Engineers, Bellingham, Washington, 1978).

5. Nyyssonen, D., Optical Linewidth Measurements on Wafers, Developments in Semiconductor Microlithography III 135, pp. 115-119 (Society of PhotoOptical Instrumentation Engineers, Bellingham, Washington, 1978).

6. Swyt, D. A., Design of a Pattern on a Photomask-Like Physical Standard for Evaluation and Calibration of Linewidth-ileasuring Systems, Solid Sta te Tech. 21, 35-42 (January 1978).

7. Nyyssonen, D., Spatial Coherence: The Key to Accurate Optical Micrometrology, Proc. Soc. Photo-Optical Instrumentation Engineers 194, Applications of Optical Coherence, 34-44 (1979).

8. Reference [2], pp. 16-35.

9. Nyyssonen, D., Linewidth Measurement with an Optical Microscope: The Effect of Operating Conditions on the Image Profile, Appl. Optics 16, No. 8, 2223-2230 (August 1977).

10. Nyyssonen, D., Linewidth Measurement Spotlight, Semiconductor International 3, No. 3, 39-56 (March 1980).

11. Reference [3], pp. 136-138.

12. Swyt, D. A., and Rosberry, F. W., A Comparison of Some Optical Microscope Measurements of Photomask Linewidths, Solid State Tech. 20, 70-75 (August 1977).

13. Einspruch, N. G., ed., VLSI Electronics - Microstructure Science, Vol. 3, Ch. 7 (Academic Press, Inc., New York, 1982).

14. Reference [4], pp. 102-104. 
15. Reference [3], pp. 135-136.

16. Reference [2], pp. 7-15.

17. Reference [3], pp. 142-144.

18. Reference [3], pp. 136-139.

19. Reference [4], pp. 99-101.

20. Reference [12], pp. 74-75.

21. Ku, H. H., ed., Precision Measurement and Calibration - Statistical Concepts and Procedures, NBS Spec. Publ. 300, Vol. 1 (February 1969).

22. Eisenhart, C., Realistic Evaluation of the Precision and Accuracy of Instrument Calibration Systems, NBS J. Res. 67C, No. 2, 161-187 (AprilJune 1963).

23. Youden, W. J., Graphical Diagnosis of Interlaboratory Test Results, Industrial Quality Control 15, 1-5 (May 1959).

24. Standard Recommended Practice for Dealing with Outlying Observations, ASTM Designation E178, Annual Book of ASTM Standards, Part 41 (November 1981). (Available as a separate reprint from American Society for Testing and Materials, 1916 Race Street, Philadelphia, PA 19103.)

25. Barnett, V., and Lewis, T., Outliers in Statistical Data (John Wiley and Sons, Inc., New York, 1978).

26. Neter, J., and Wasserman, W., Applied Linear Statistical Models, pp. 21-48 (Richard D. Irwin, Inc., Homewood, Illinois, 1974).

27. Reference [26], pp. 113-121.

28. Lieberman, G. J., Miller, R. G., and Hamilton, M. A., Unlimited Simultaneous Discrimination Intervals in Regression, Biometrika 54, 133145 (1967).

29. Scheffe, H., Rosenblatt, J., and Spiegelman, C., A Statistical Theory of Calibration (to be published).

30. Reference [26], pp. 53-60.

31. Graybill, F. A., An Introduction to Linear Statistical Models, Vol. 1, pp. 106-148 (McGraw Hill Book Company, Inc., New York, 1961).

32. Reference [10], pp. 39-40.

33. Reference [1], p. 256. 
34. Reference $[10]$, p. 47.

35. Reference [6], p. 38 .

36. Croarkin, M. C., and Varner, R. N., Measurement Assurance for Dimensional Measurements on Integrated-Circuit Photomasks, NBS Tech. Note 1164 (August 1982). 


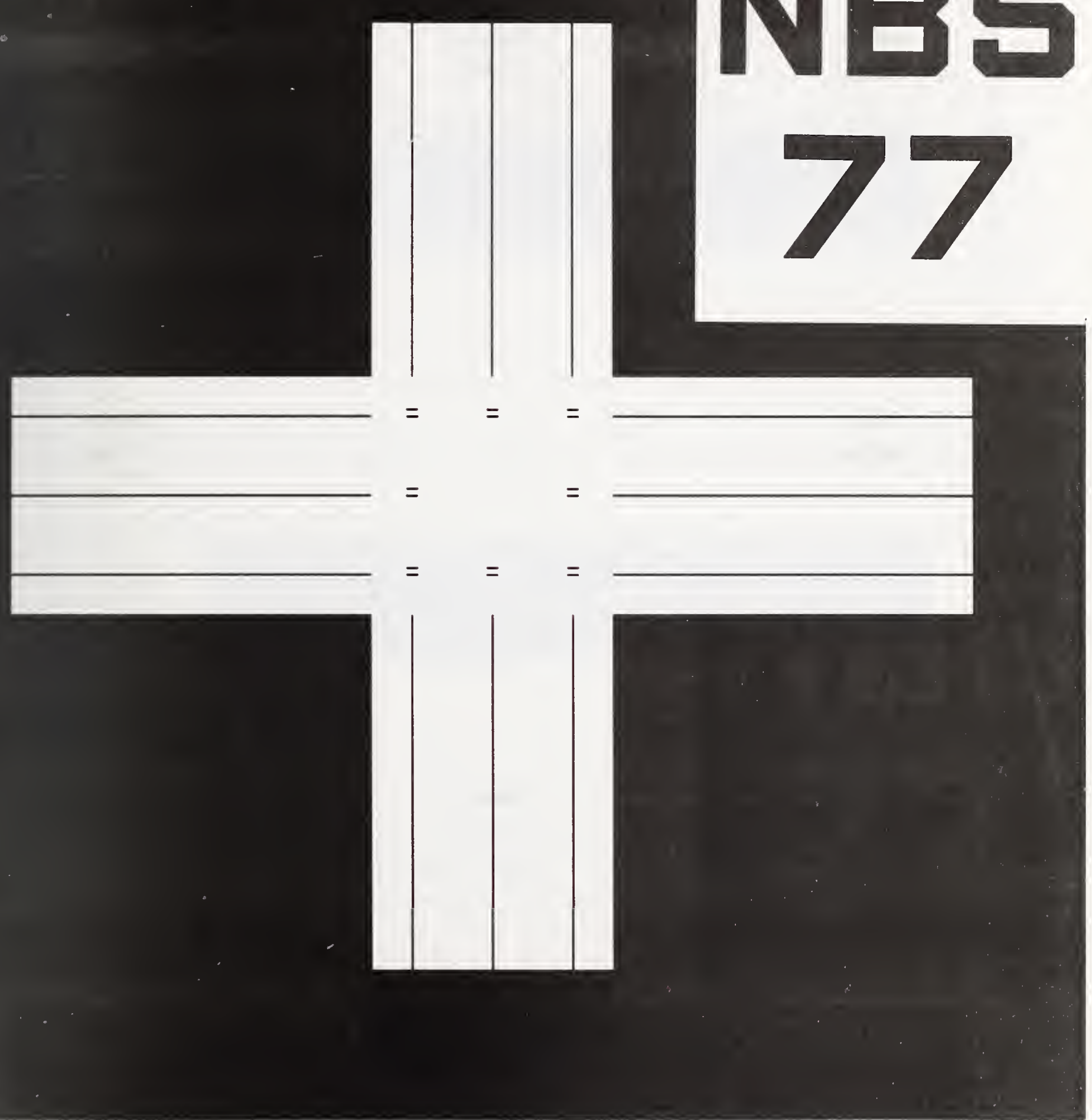

Figure 1. Photograph of overall pattern on NBS antireflective-chromium artifact at a magnification of about 3.4x. (The horizontal and vertical ines help to locate the eight basic measurement patterns in the center.) 

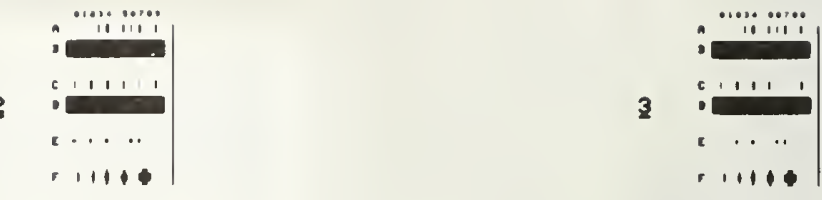

, 1110

1110

, 1111
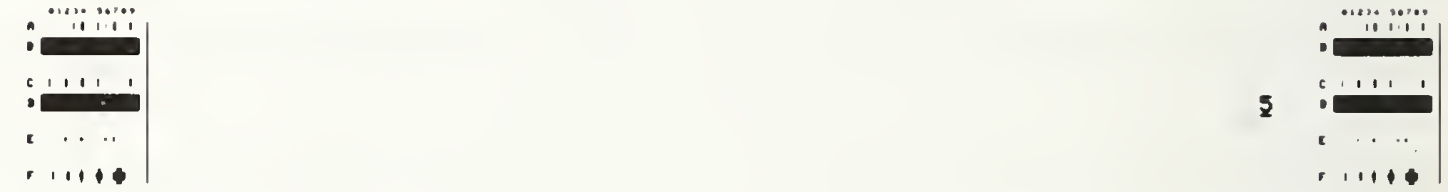

1110

$+1+\bullet$

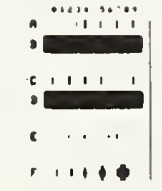

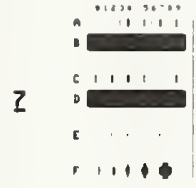

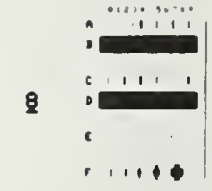

Figure 2. Photograph of central area of NBS antireflective-chromium artifact at a magnification of about 16x. (The basic measurement pattern is repeated eight times about the center, and each basic pattern is numbered. A number of the smallest lines and spaces in each pattern do not appear in this photograph.) 


\section{田1294 56799}

$\overline{\mathrm{H}}-1-1-1 \cdot 1|-|-|-|-\mid-1-$

$E:|-|-|-|-|--|-|-|-|-|-$

L. $-|-|-|-|-|--|-|-|-|-|-$

II $-|-|-|-|-|--|-|-|-|-|-$
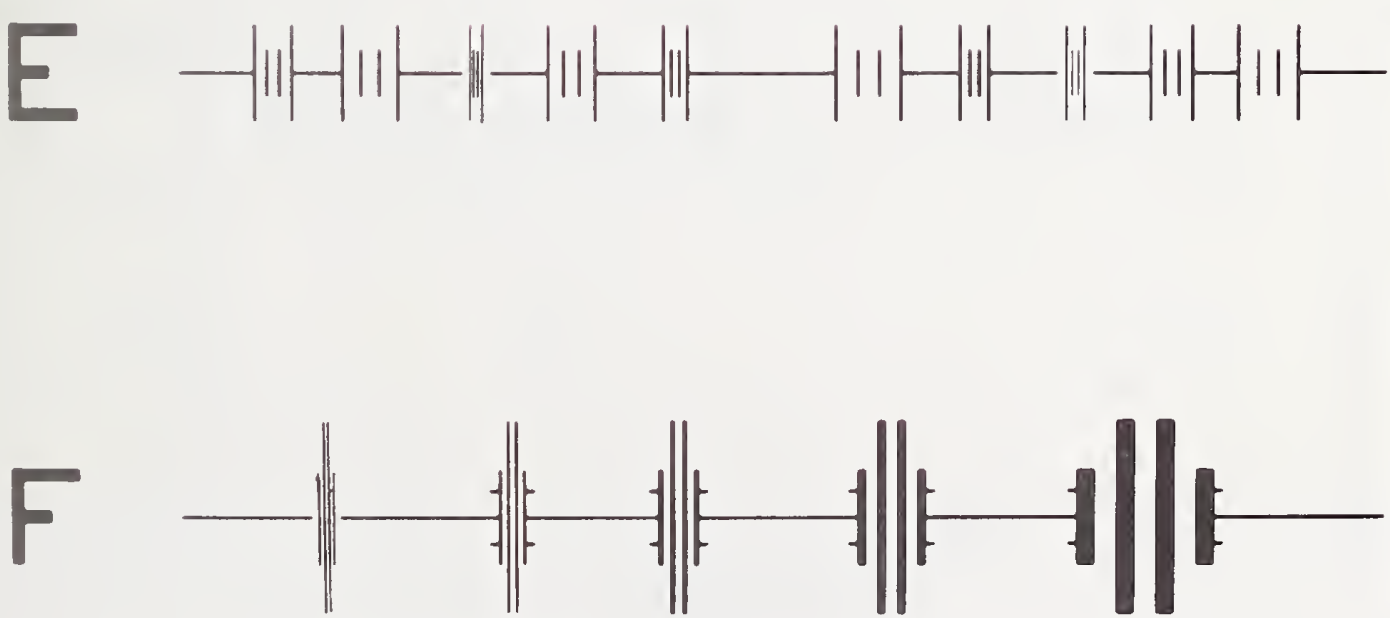

Figure 3. Photograpil of basic measurement pattern on NBS antireflective-chromium artifact at a magnification of about 200x. (The individual lines and patterns are located by reference to an alphanumeric grid with the letters $A$ through $F$ identifying the row and the numbers 0 through 9 identifying the column.) 


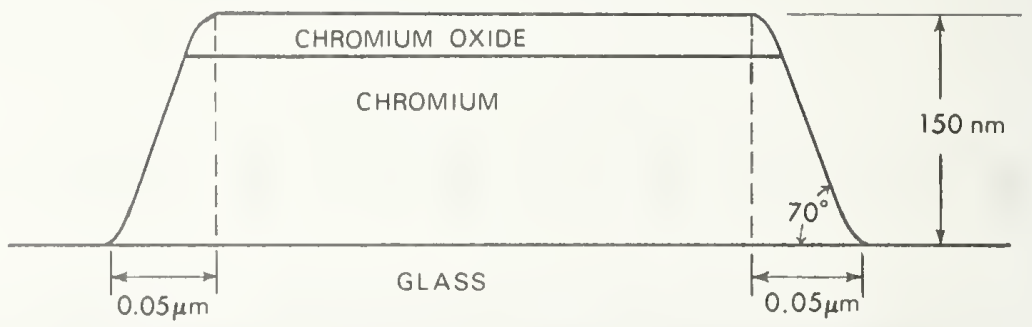

Figure 4. Schematic of physical profile of opaque line on NBS antireflectivechromium artifact. 


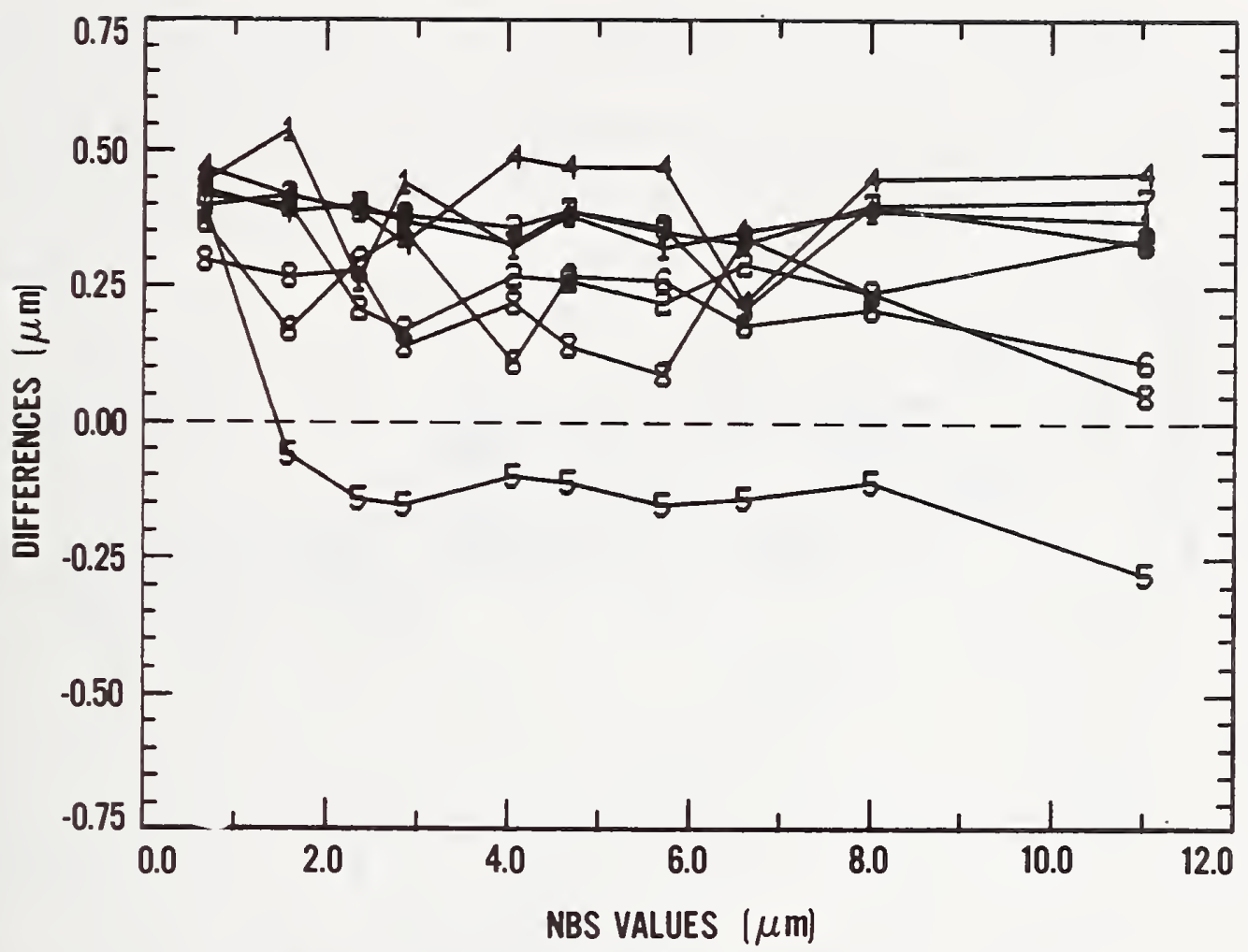

(a) One day's measurements (data set 5) out of statistical control.

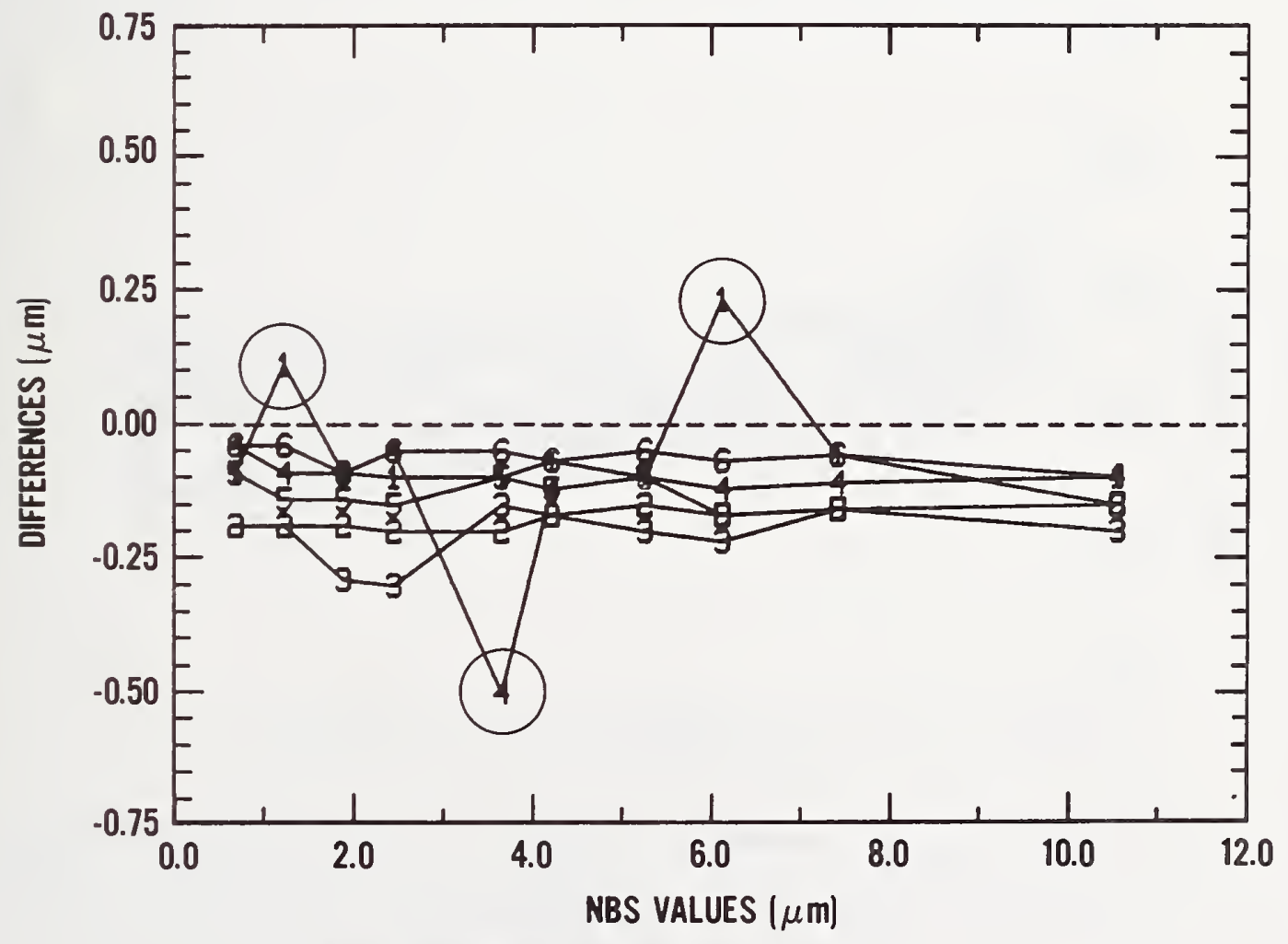

(b) Significant isolated outliers. (Outliers are circled.)

Figure 5. Examples of differences between participant's linewidth measurements and NBS linewidth values as a function of NBS values. (Each number represents one set of measurements.) 


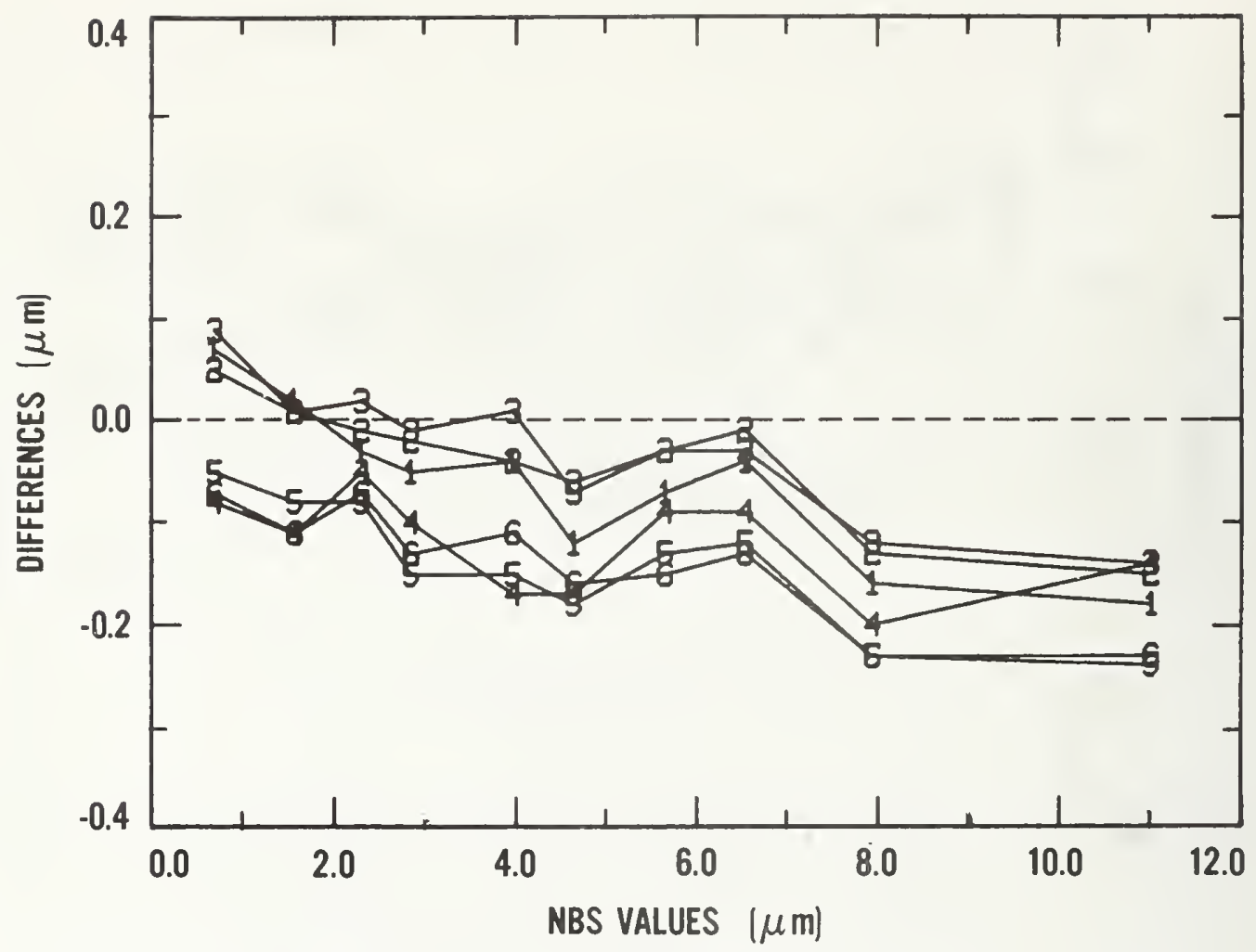

(c) Significant differences between two operators. (Data sets 1, 2, and 3 are for the first operator, and data sets 4, 5, and 6 are for the second operator.)

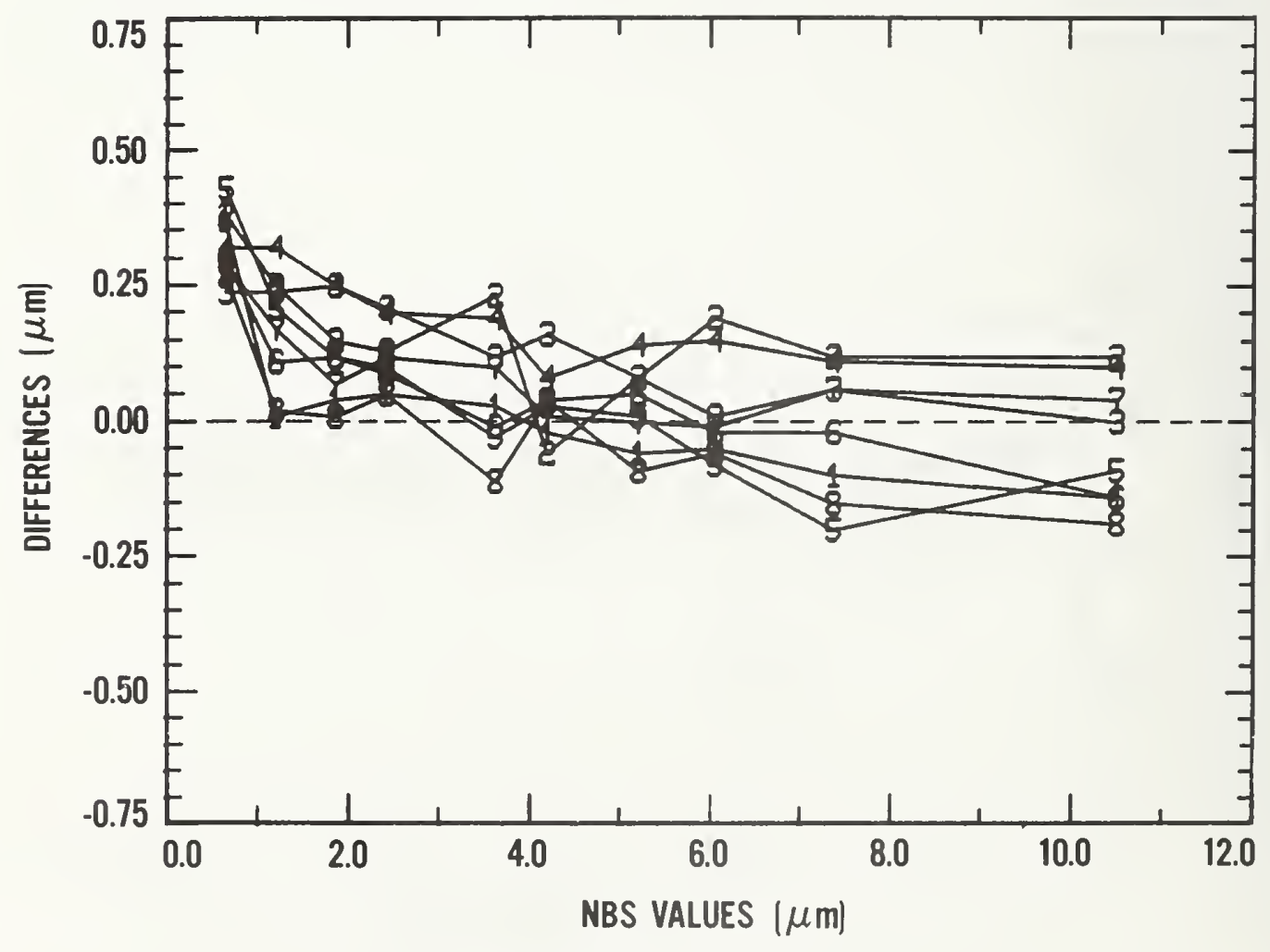

(d) Nonlinear relationship.

Figure 5. Concluded. 


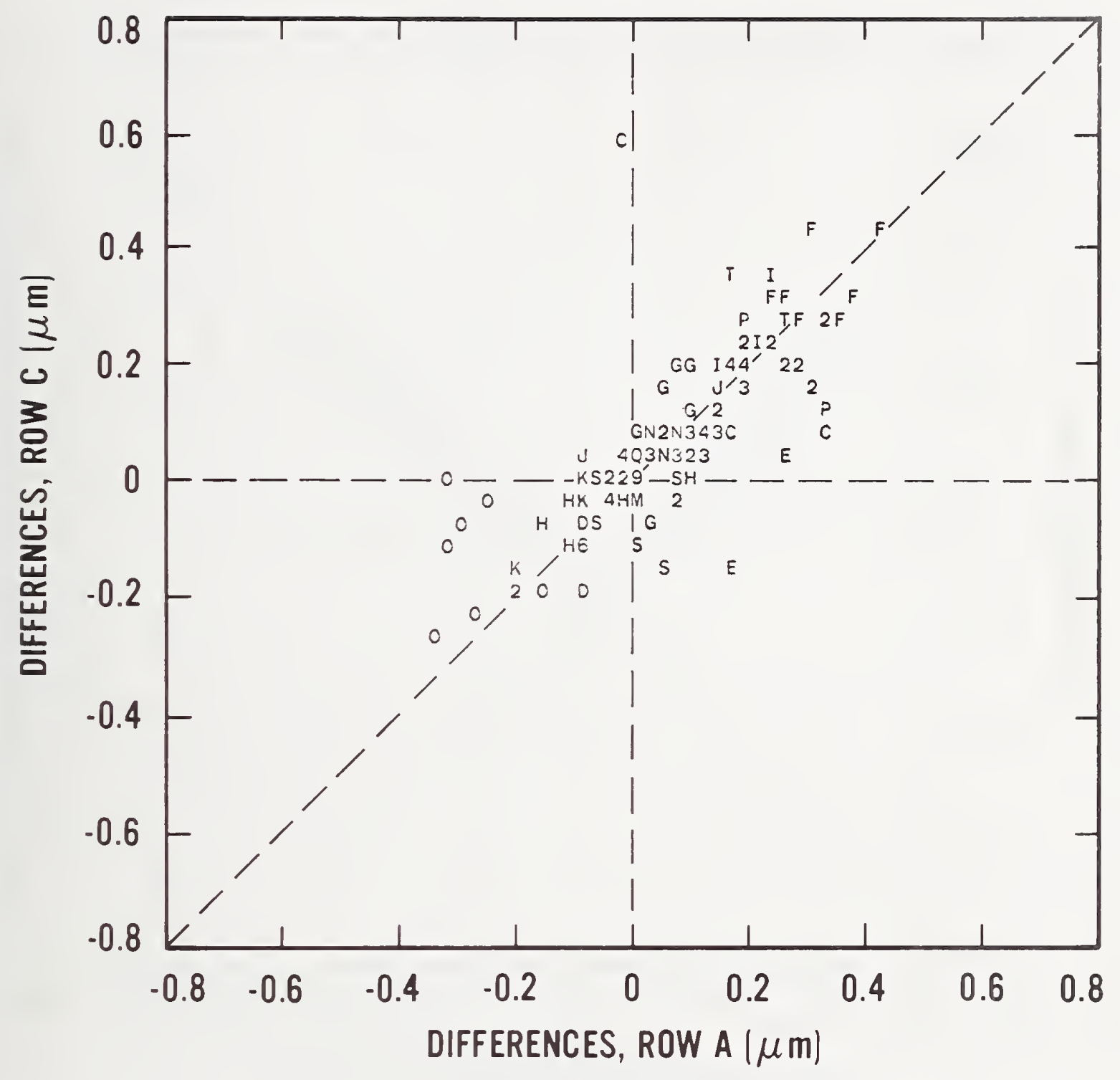

a. Lines $A-2$ with mean NBS value of $0.71 \mu \mathrm{m}$ and lines $\mathrm{C}-3$ with mean NBS value of $0.73 \mathrm{\mu m}$.

Figure 6. Youden plots of differences between participants' raw linewidth measurements on opaque lines (rows A and C) and NBS values. (Letters A through $\mathrm{T}$ represent different measurement systems as identified in table 3.) 


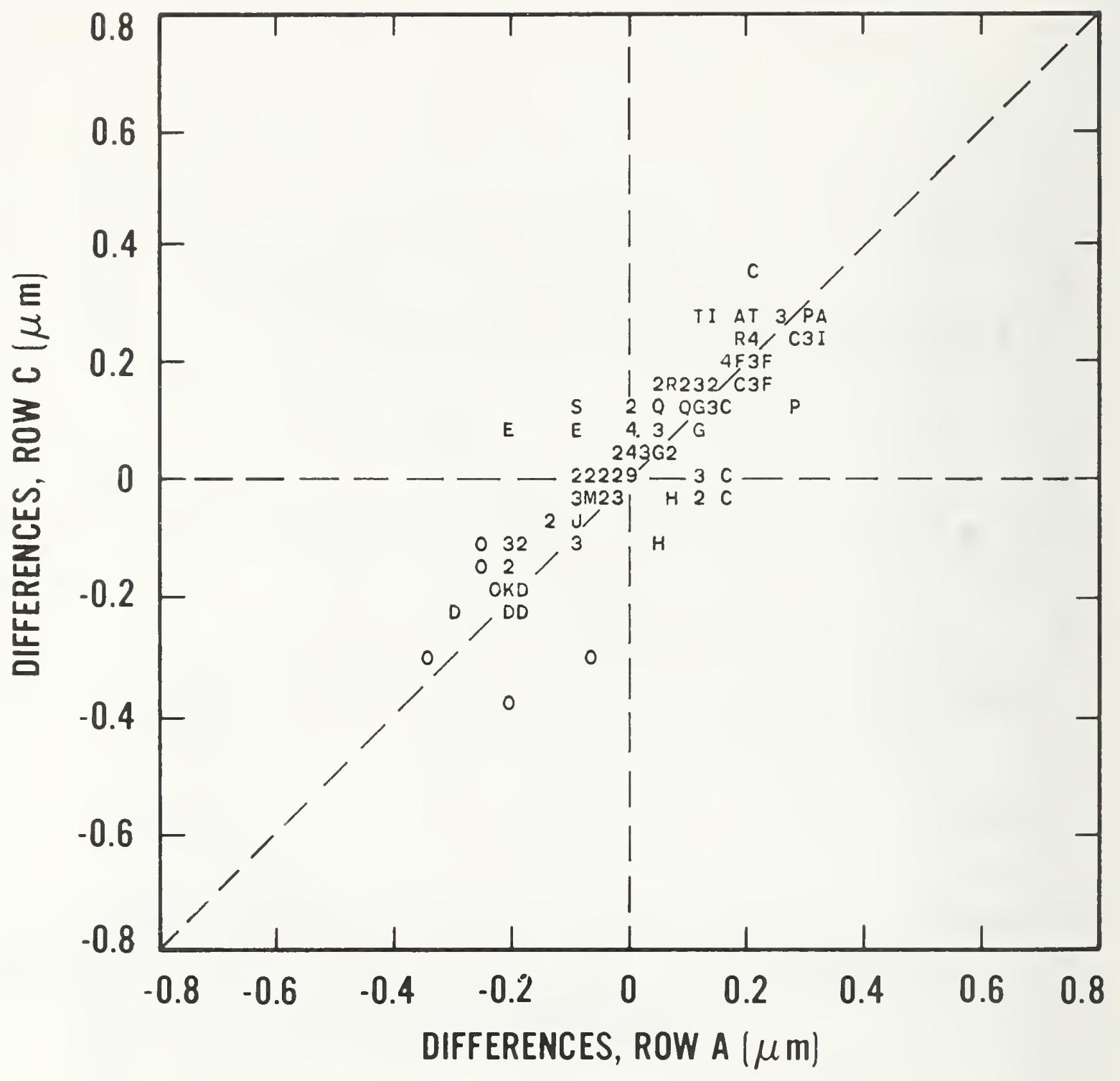

b. Lines A-8 with mean NBS value of $1.27 \mu \mathrm{m}$ and

lines C-6 with mean NBS value of $1.22 \mu \mathrm{m}$.

Figure 6 Continued. 


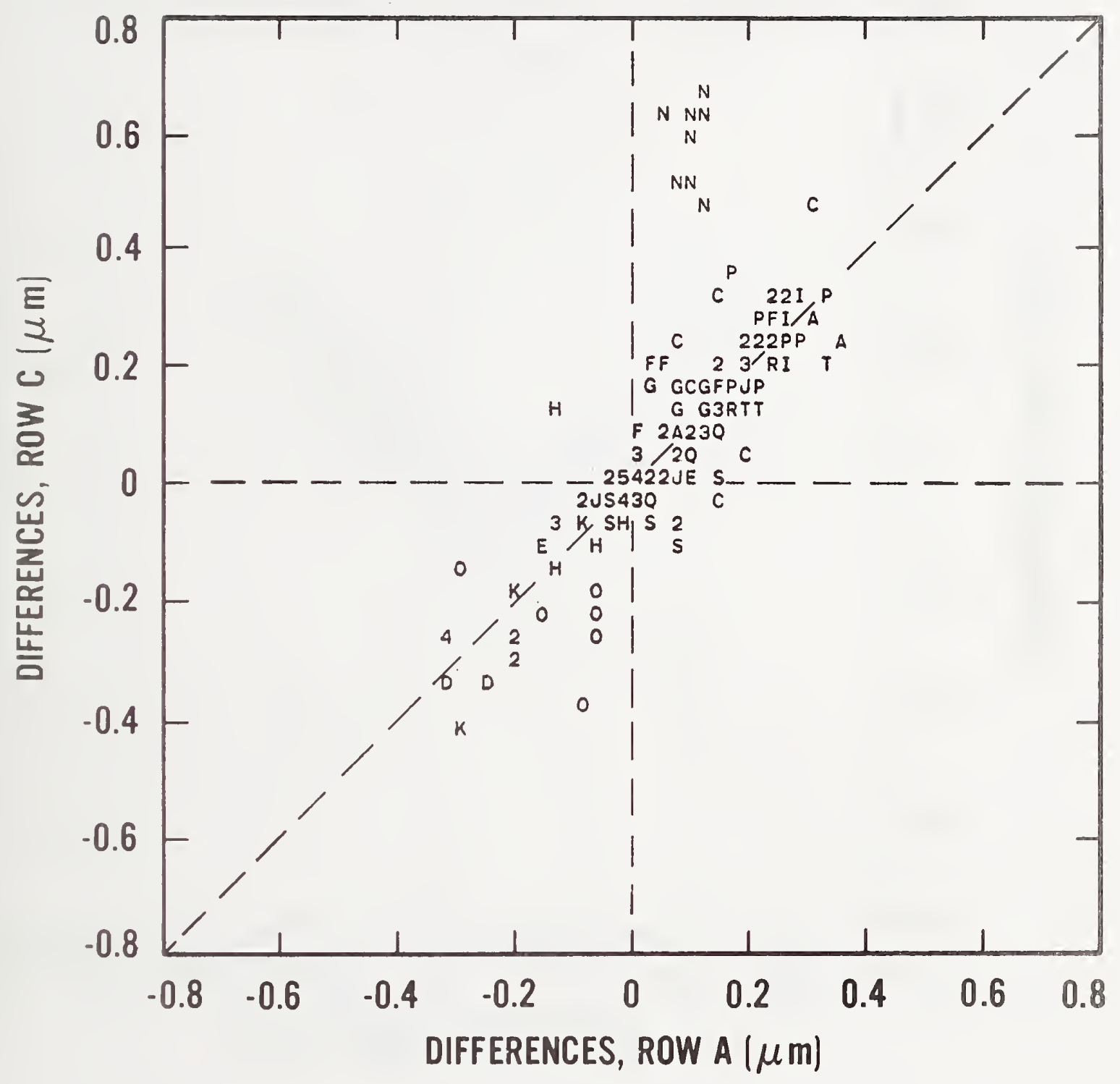

c. Lines A-1 with mean NBS value of $1.91 \mu \mathrm{m}$ and lines $C-1$ with mean NBS value of $1.70 \mu \mathrm{m}$.

Figure 6 Continued. 


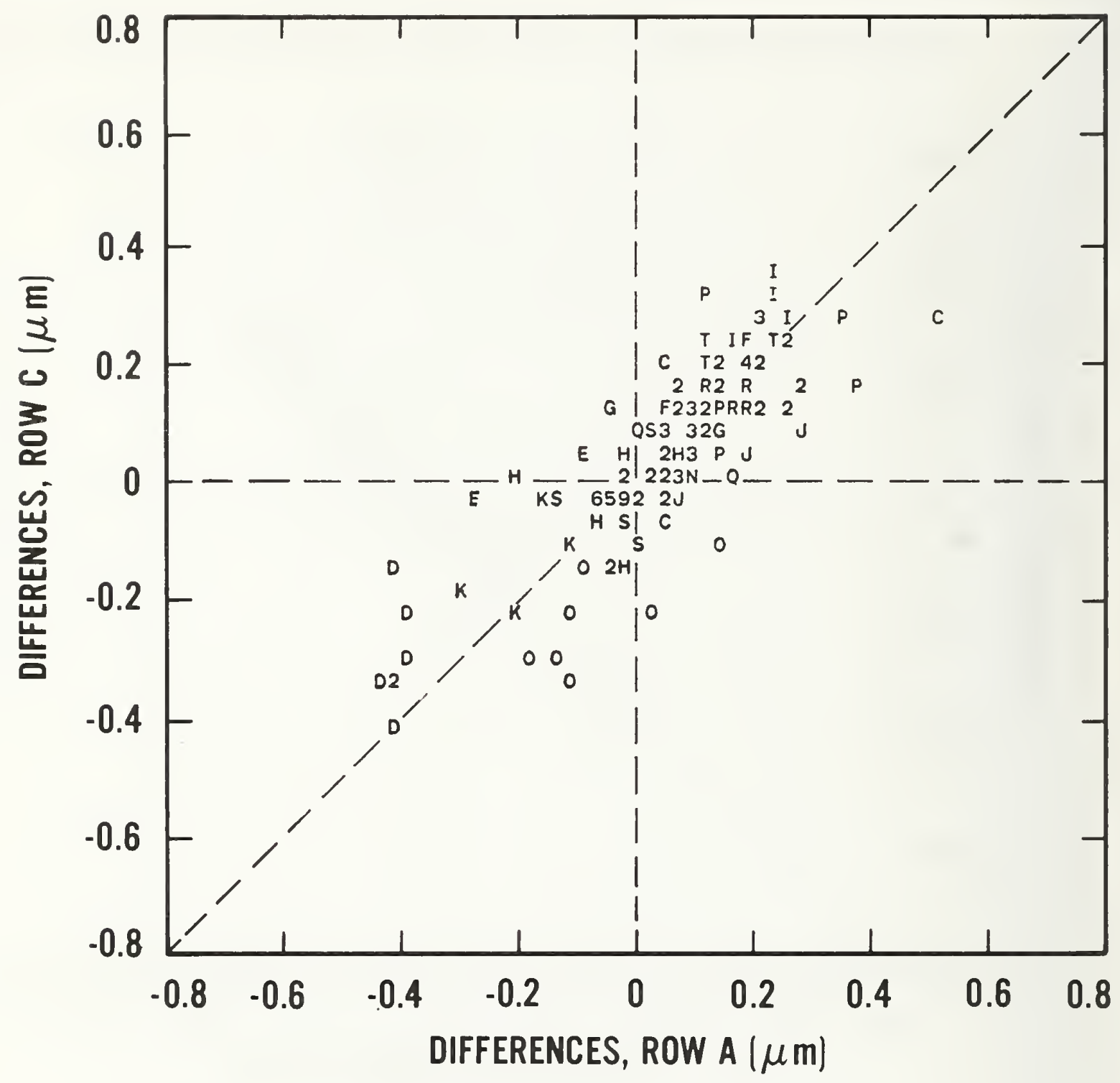

d. Lines A-0 with mean NBS value of $2.47 \mu \mathrm{m}$ and lines $\mathrm{C}-8$ with mean NBS value of $2.05 \mu \mathrm{m}$.

Figure 6 Continued. 


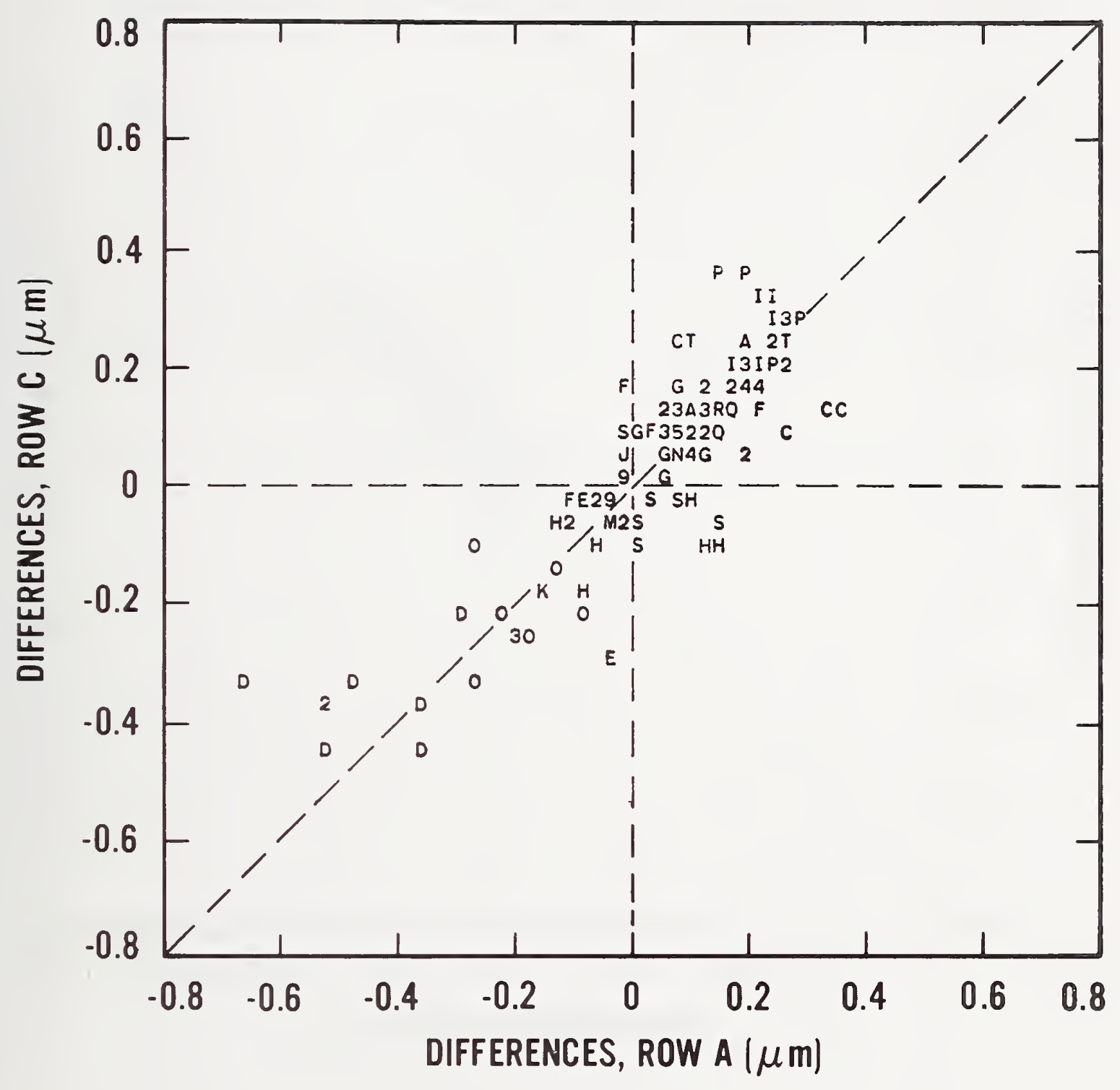

e. Lines A-6 with mean NBS value of $3.66 \mu \mathrm{m}$ and lines $\mathrm{C}-7$ with mean NBS value of $3.27 \mu \mathrm{m}$.

Figure 6 Continued. 


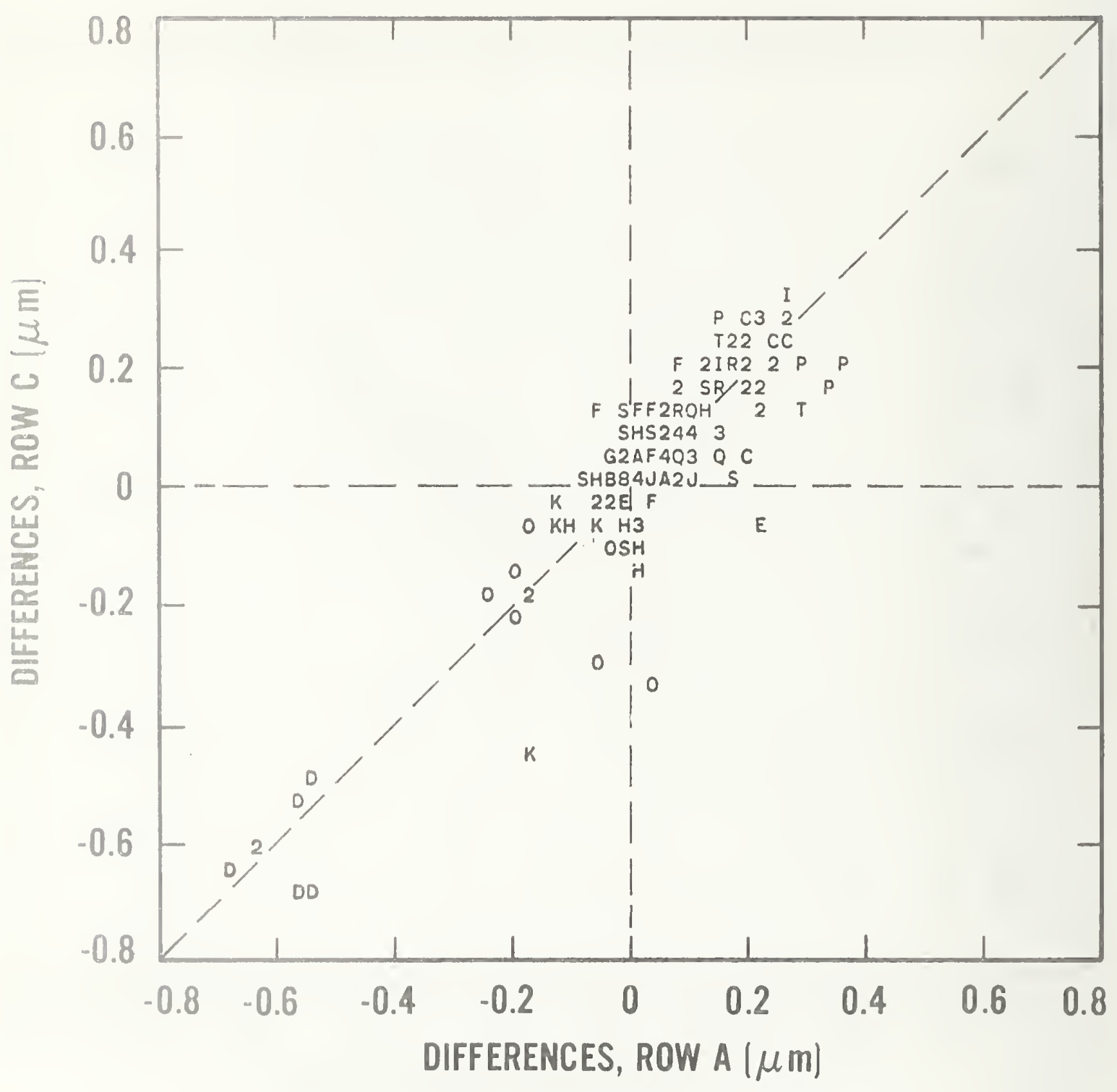

E. Lines A-3 with mean NBS value of $4.25 \mu \mathrm{m}$ and lines $C-0$ with mean NBS value of $4.70 \mu \mathrm{m}$.

Figure 6 Continued. 


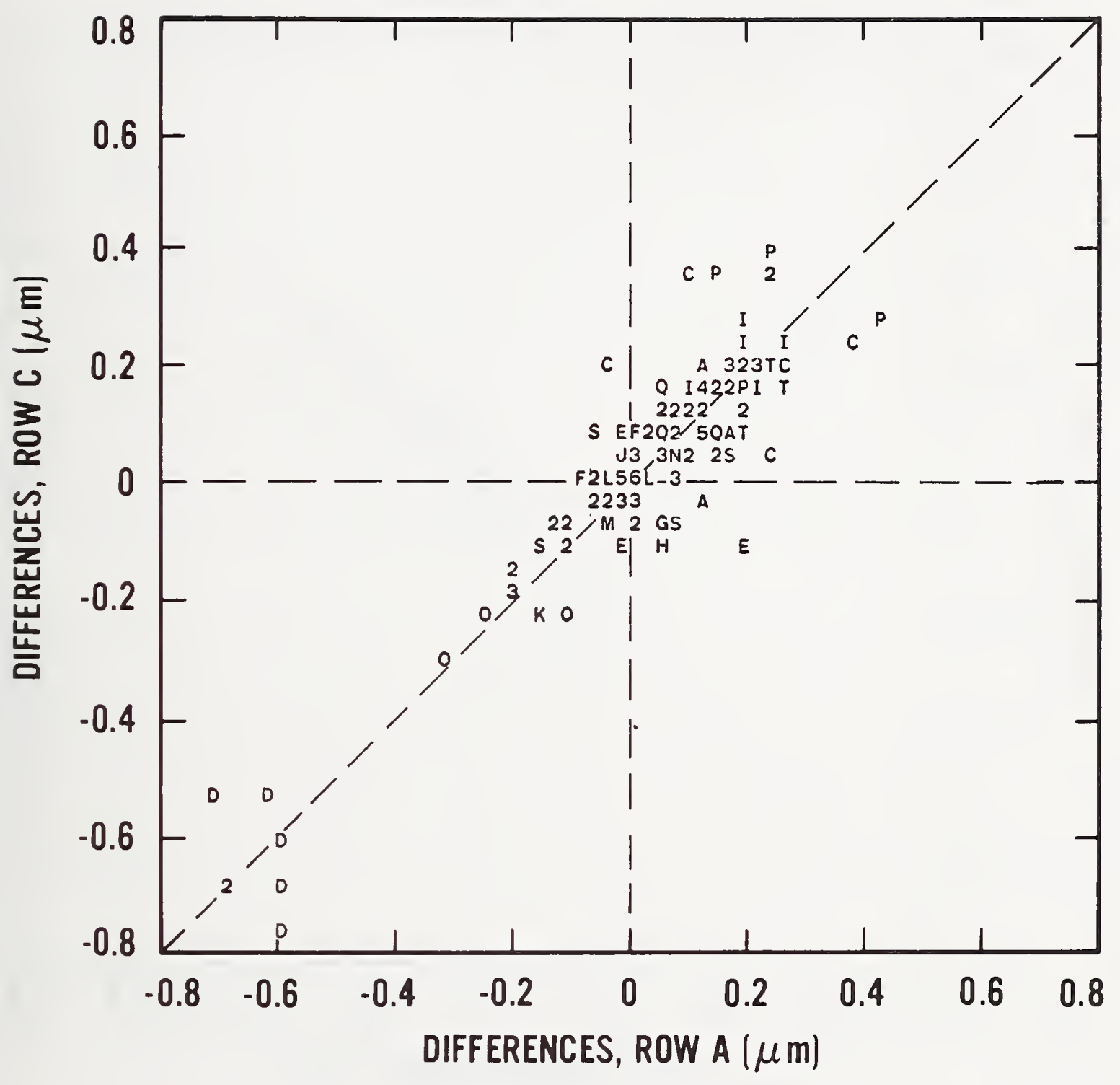

g. Lines $A-5$ with mean NBS value of $5.27 \mu \mathrm{m}$ and lines $C-5$ with mean NBS value of $5.22 \mu \mathrm{m}$

( 1 point fell outside of plotting range).

Figure 6 Continued. 


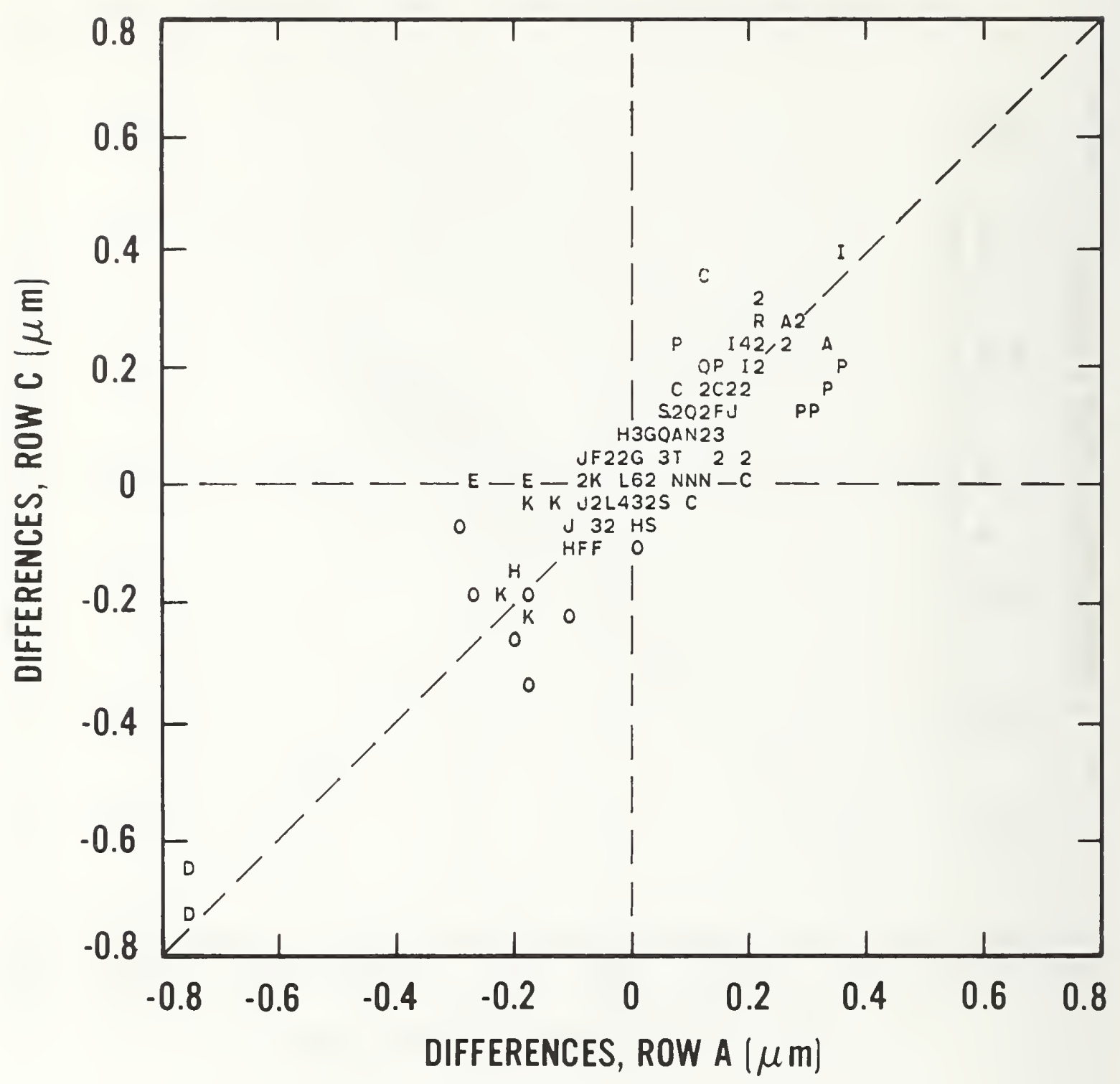

h. Lines A-9 with mean NBS value of $6.13 \mu \mathrm{m}$ and

lines C-9 with mean NBS value of $6.72 \mu \mathrm{m}$

( 6 points fell outside of plotting range).

Figure 6 Continued. 


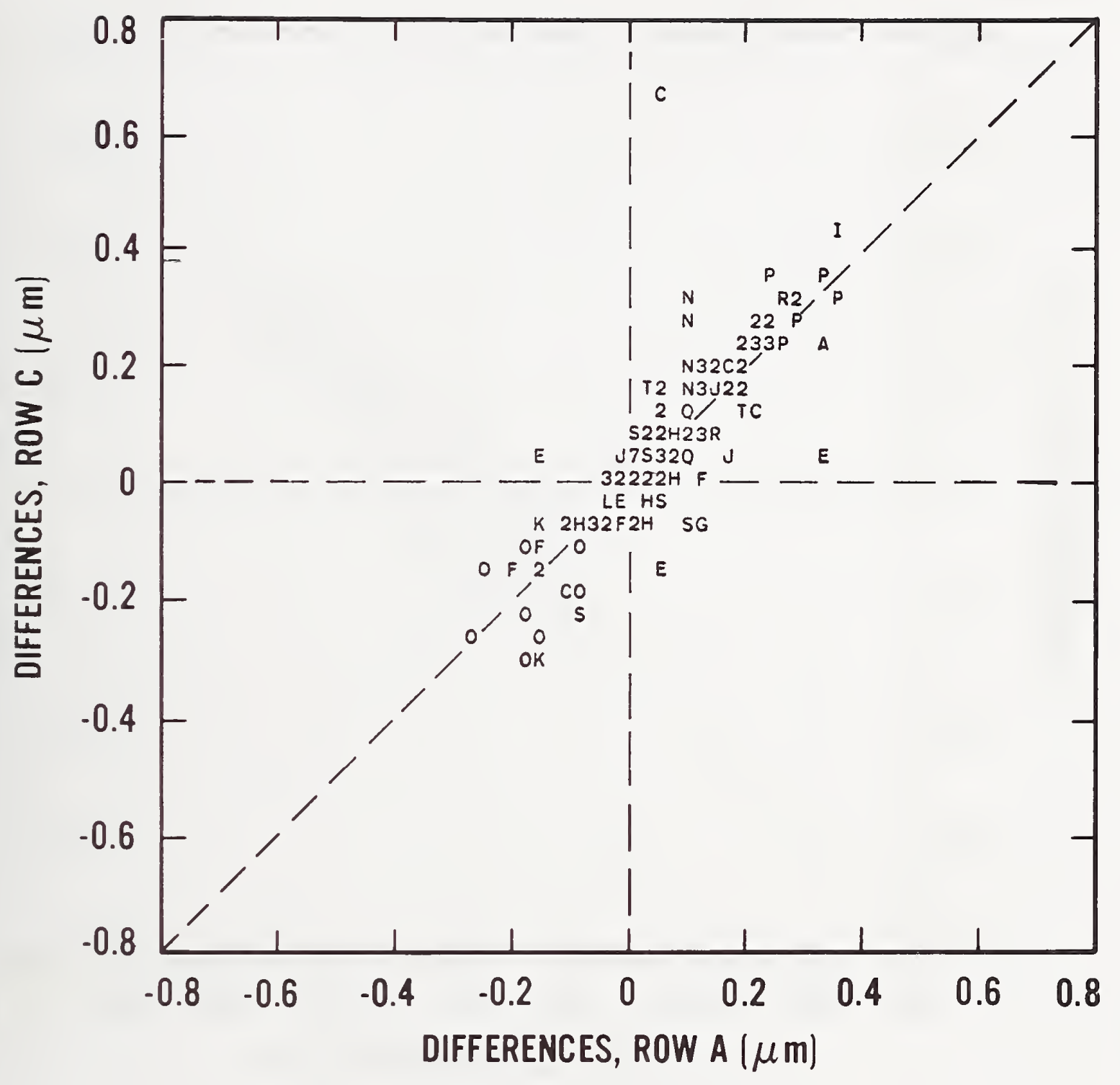

i. Lines $A-7$ with mean NBS value of $7.42 \mu \mathrm{m}$ and lines $\mathrm{C}-2$ with mean NBS value of $8.23 \mu \mathrm{m}$ ( 8 points fell outside of plotting range).

Figure 6 Continued. 


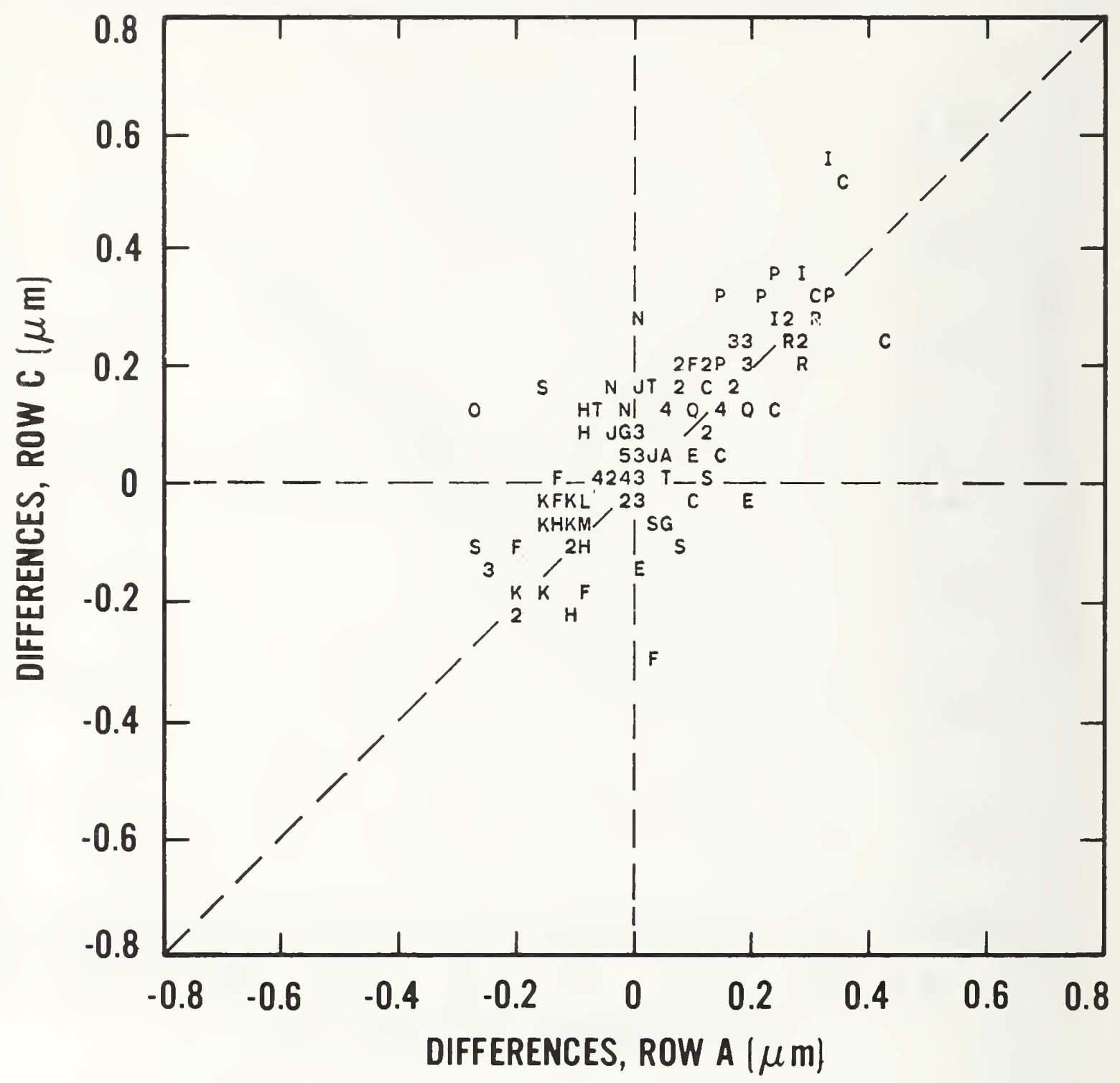

j. Lines $A-4$ with mean NBS value of $10.54 \mu \mathrm{m}$ and lines $C-4$ with mean NBS value of $8.85 \mu \mathrm{m}$

( 9 points fell outside of plotting range).

Figure 6 Concluded. 


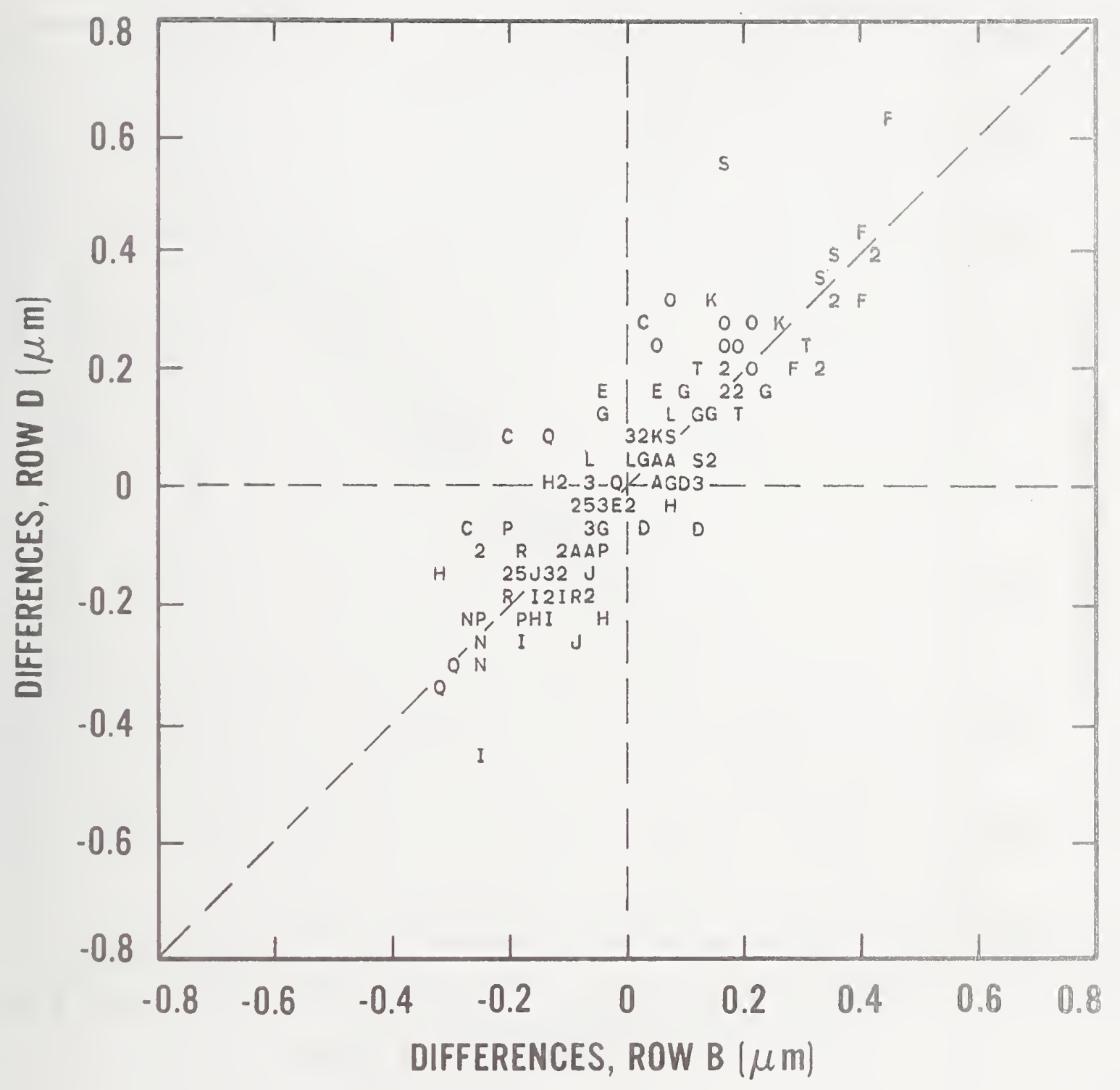

a. Lines B-7 with mean NBS value of $0.72 \mu \mathrm{m}$ and lines D-6 with mean NBS value of $0.97 \mu \mathrm{m}$.

Figure 7. Youden plots of differences between participants' raw linewidth measurements on clear lines (rows B and D) and NBS values. (Letters $A$ through $T$ represent different measurement systems as identified in table 3.) 


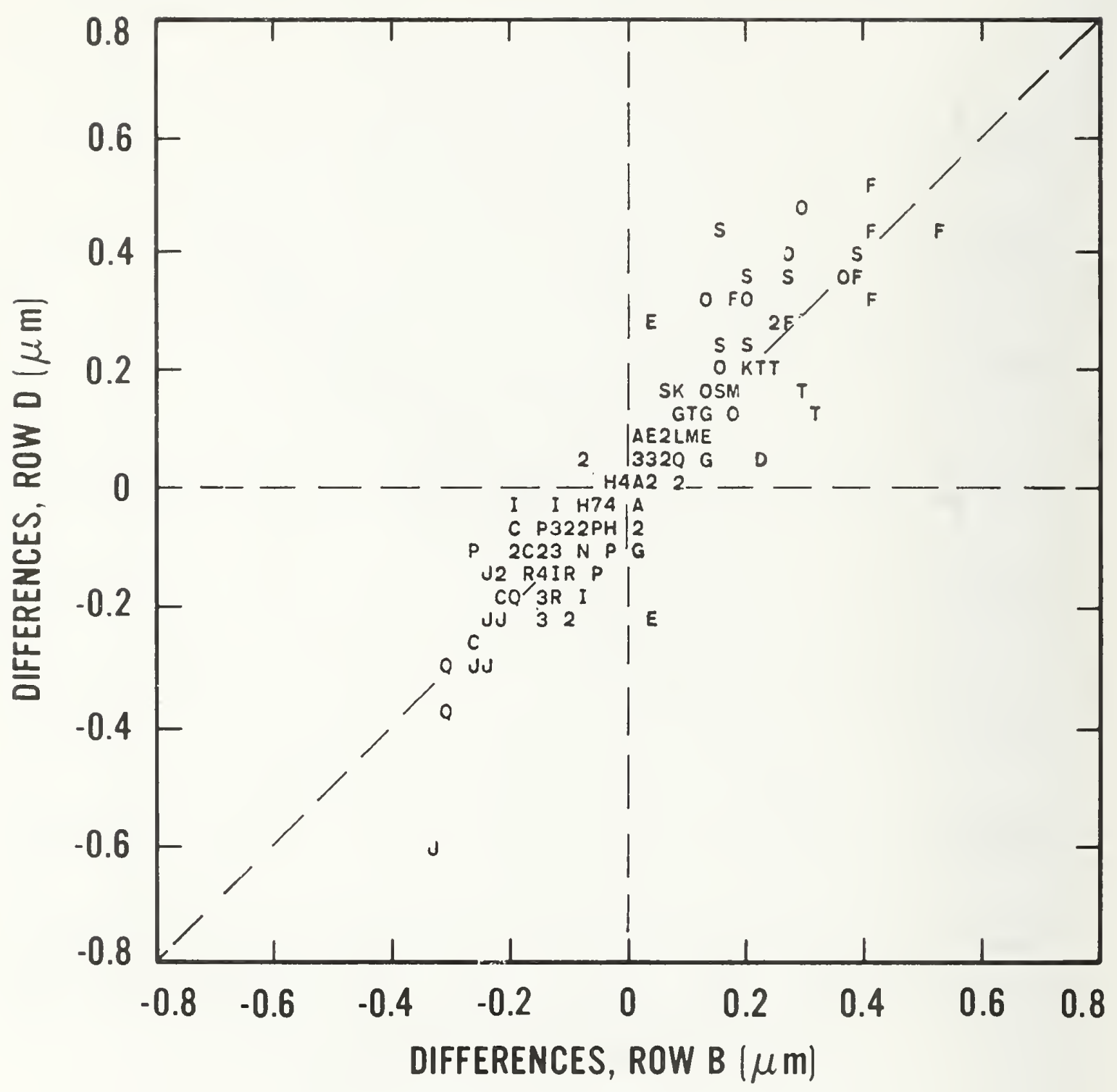

b. Lines B-1 with mean NBS value of $1.55 \mu \mathrm{m}$ and lines $D-3$ with mean NBS value of $1.53 \mu \mathrm{m}$.

Figure 7 Continued. 


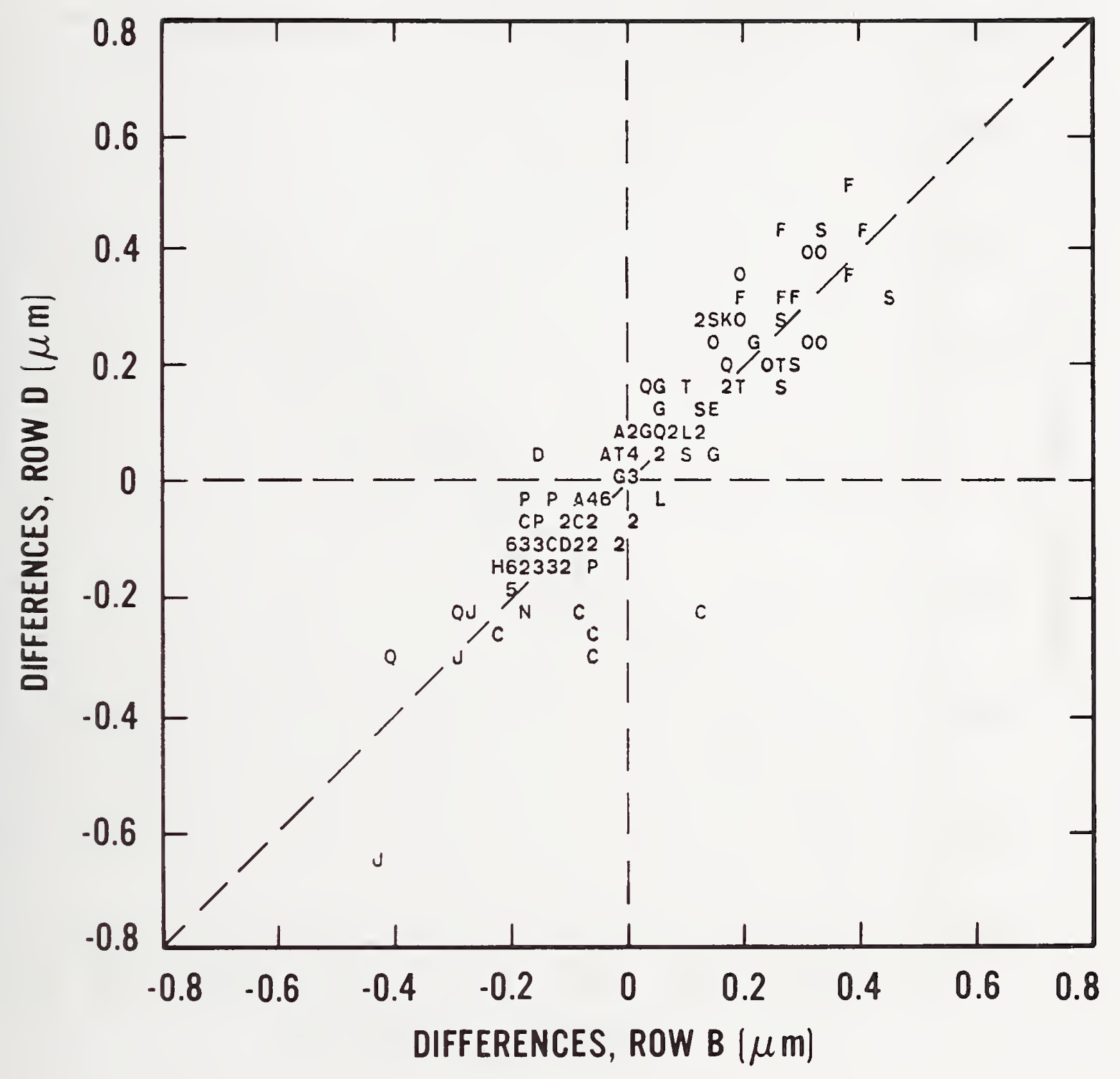

C. Lines B-8 with mean NBS value of $2.30 \mu \mathrm{m}$ and
lines D-8 with mean NBS value of $2.10 \mu \mathrm{m}$.

Figure 7 Continued. 


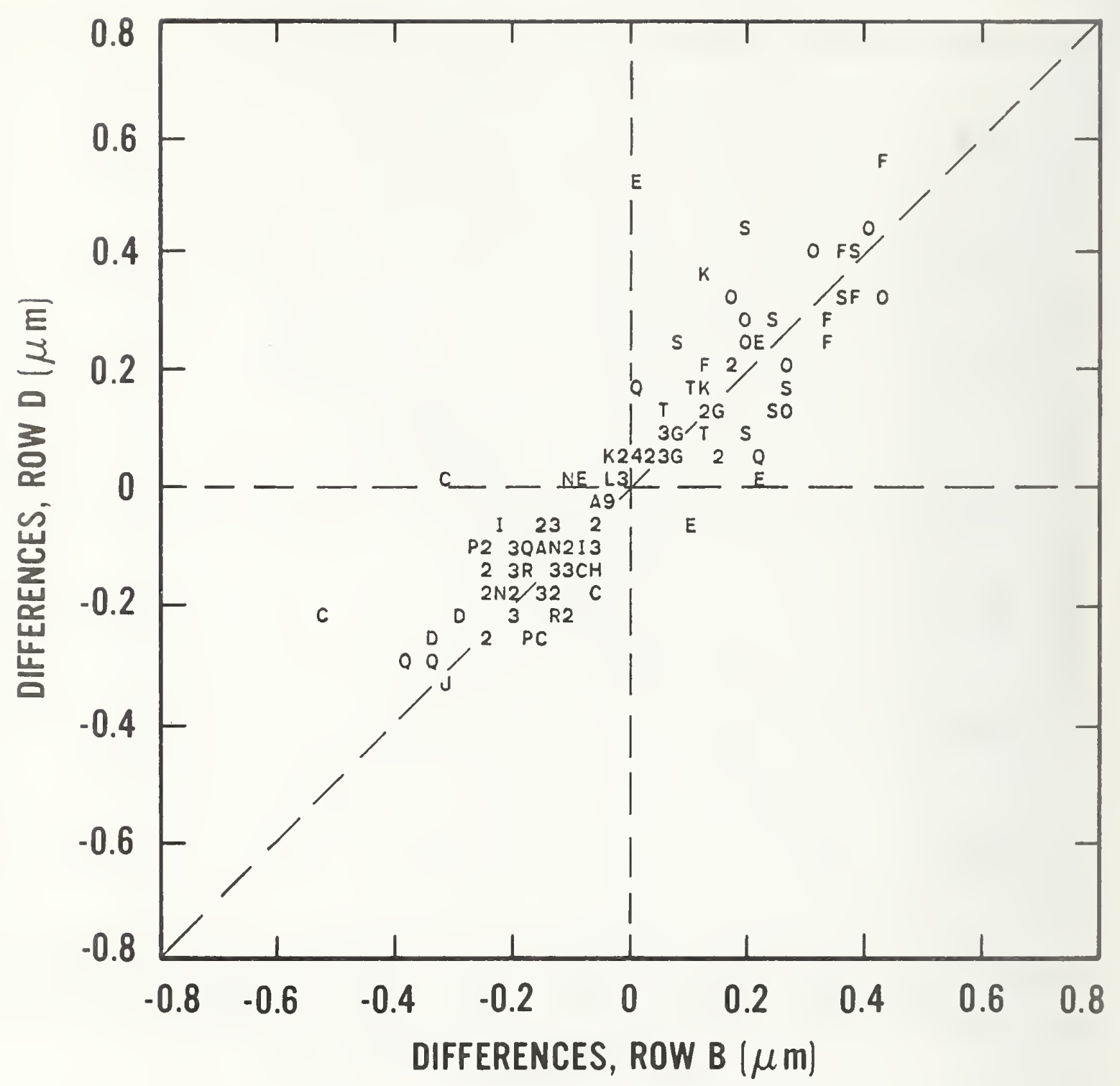

d. Lines B-9 with mean NBS value of $2.81 \mu \mathrm{m}$ and lines D-1 with mean NBS value of $2.40 \mu \mathrm{m}$.

Figure 7 Continued. 


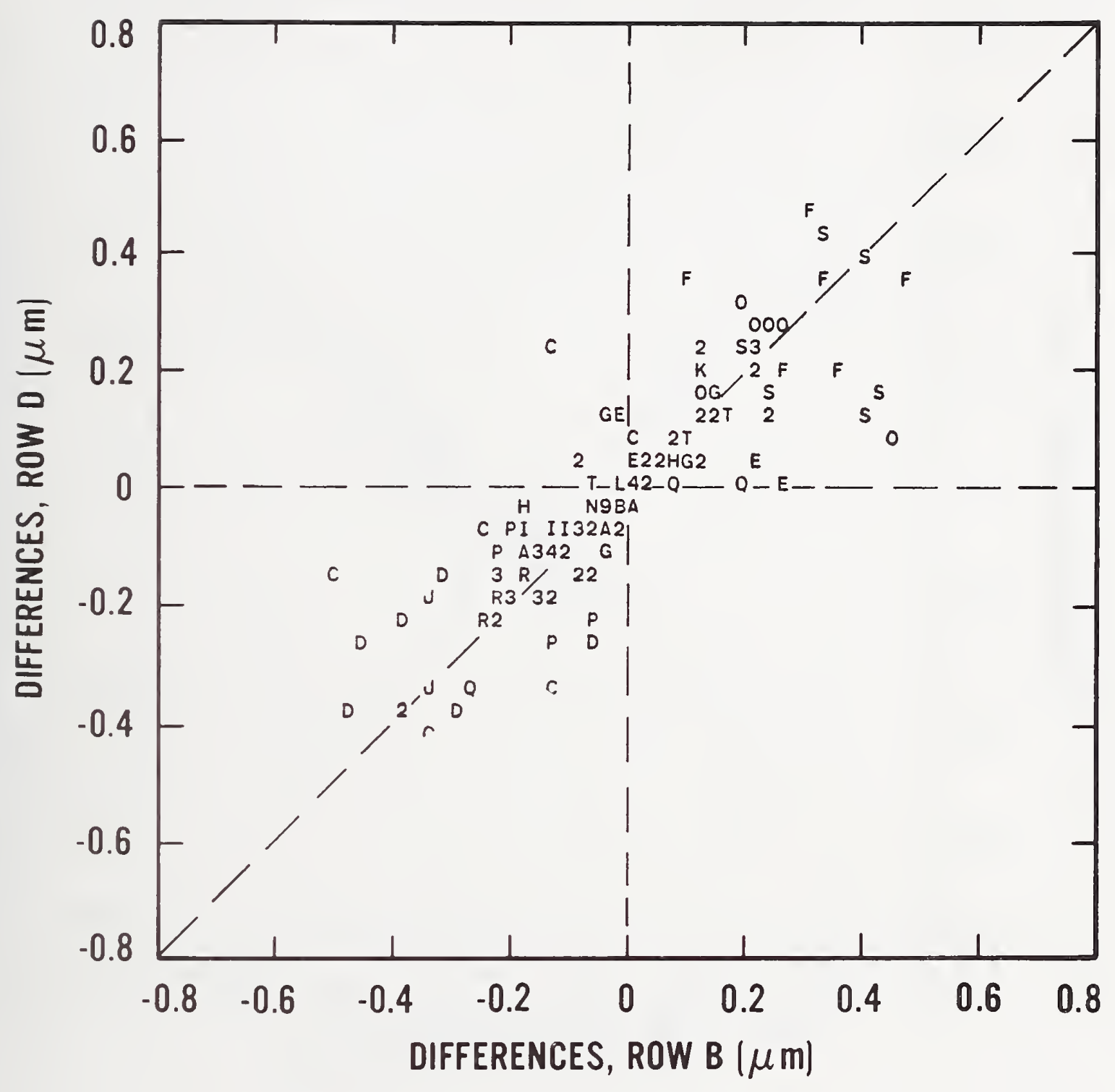

Lines B-3 with mean NBS value of $4.00 \mu \mathrm{m}$ and
lines D-2 with mean NBS value of $3.60 \mu \mathrm{m}$.

Figure 7 Continued. 


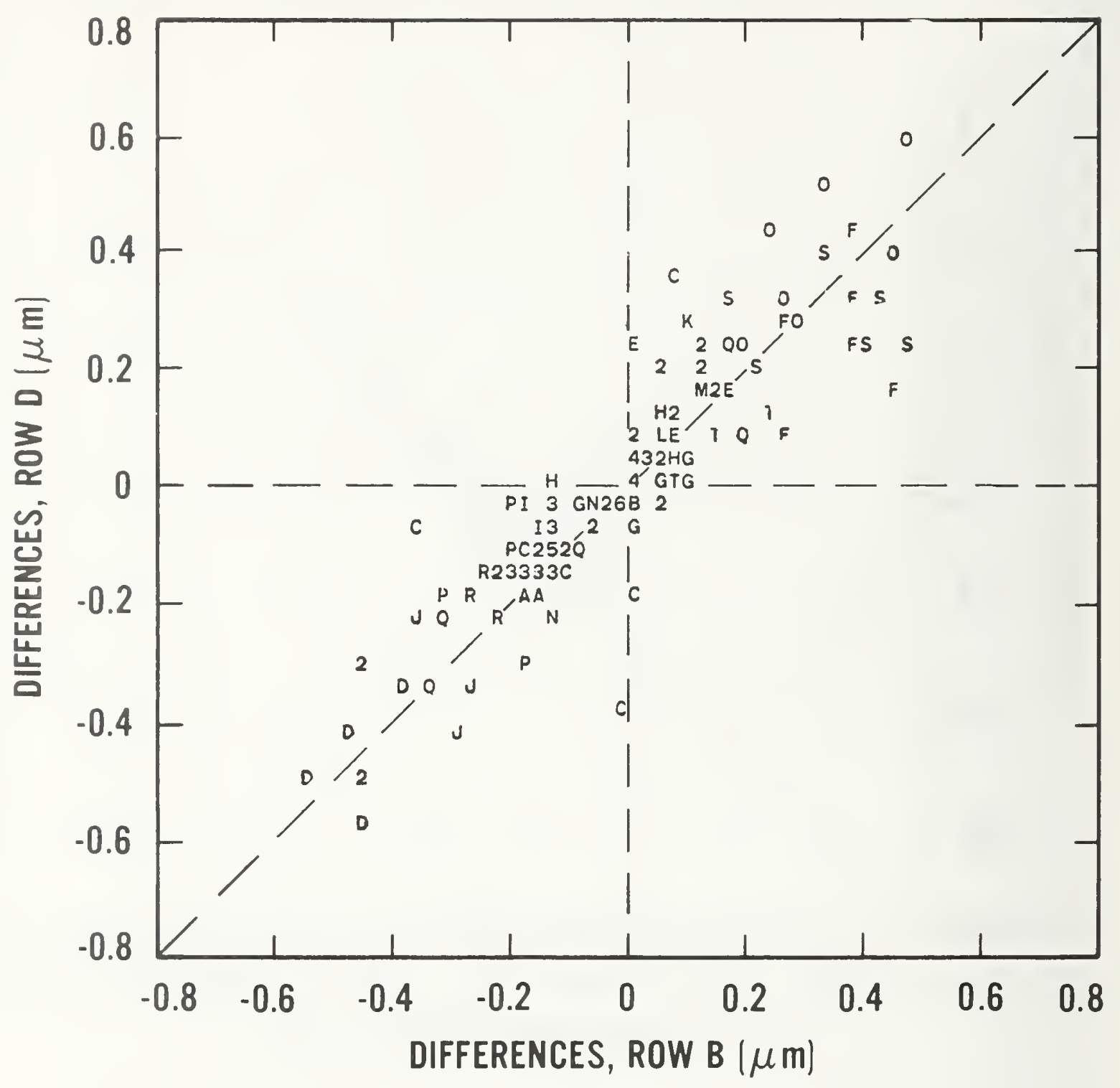

f. Lines B-6 with mean NBS value of $4.62 \mu \mathrm{m}$ and lines D-9 with mean NBS value of $5.03 \mu \mathrm{m}$.

Figure 7 Continued. 


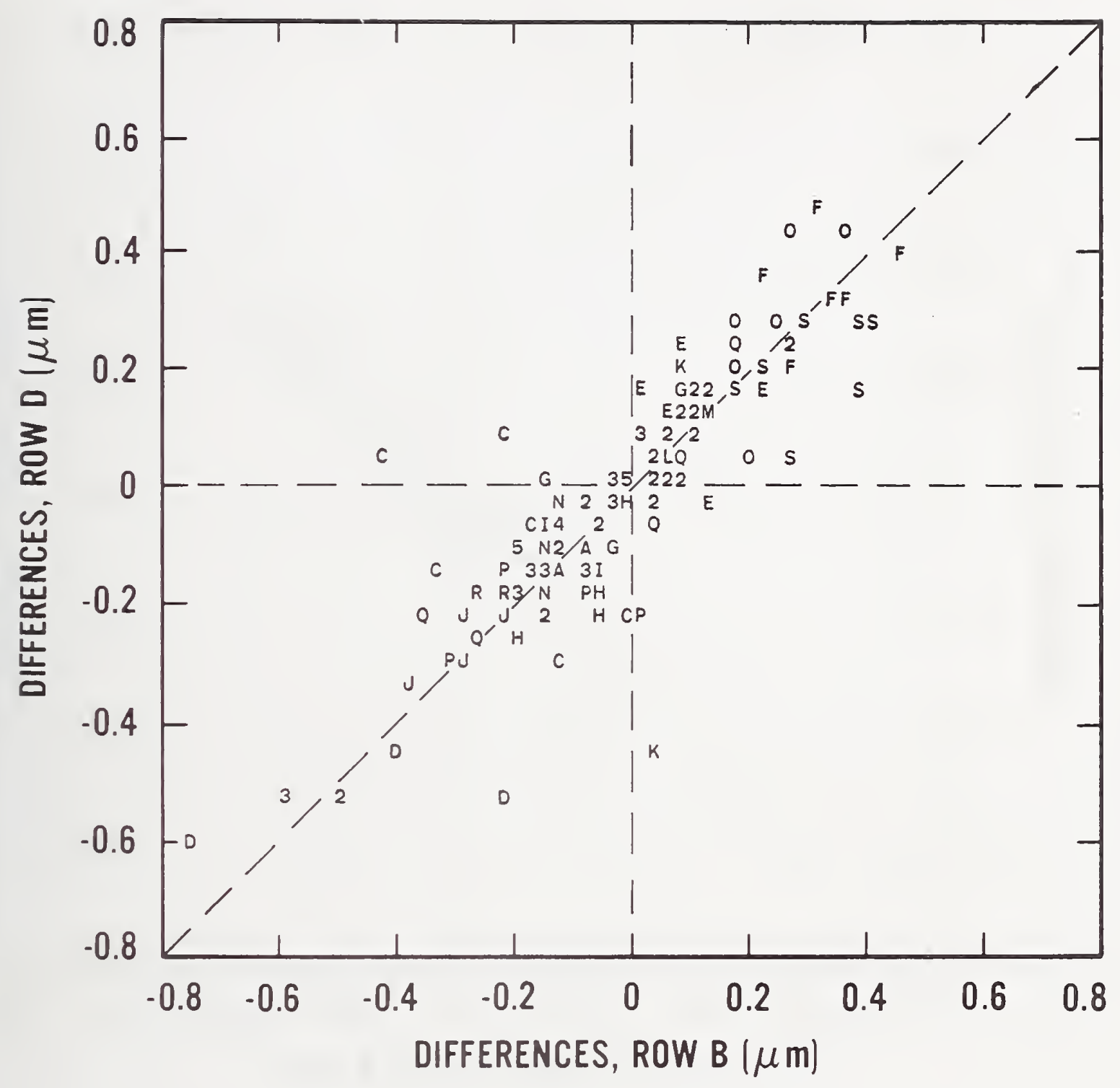

g. Lines B-4 with mean NBS value of $5.64 \mu \mathrm{m}$ and lines D-4 with mean NBS value of $5.67 \mu \mathrm{m}$.

Figure 7 Continued. 


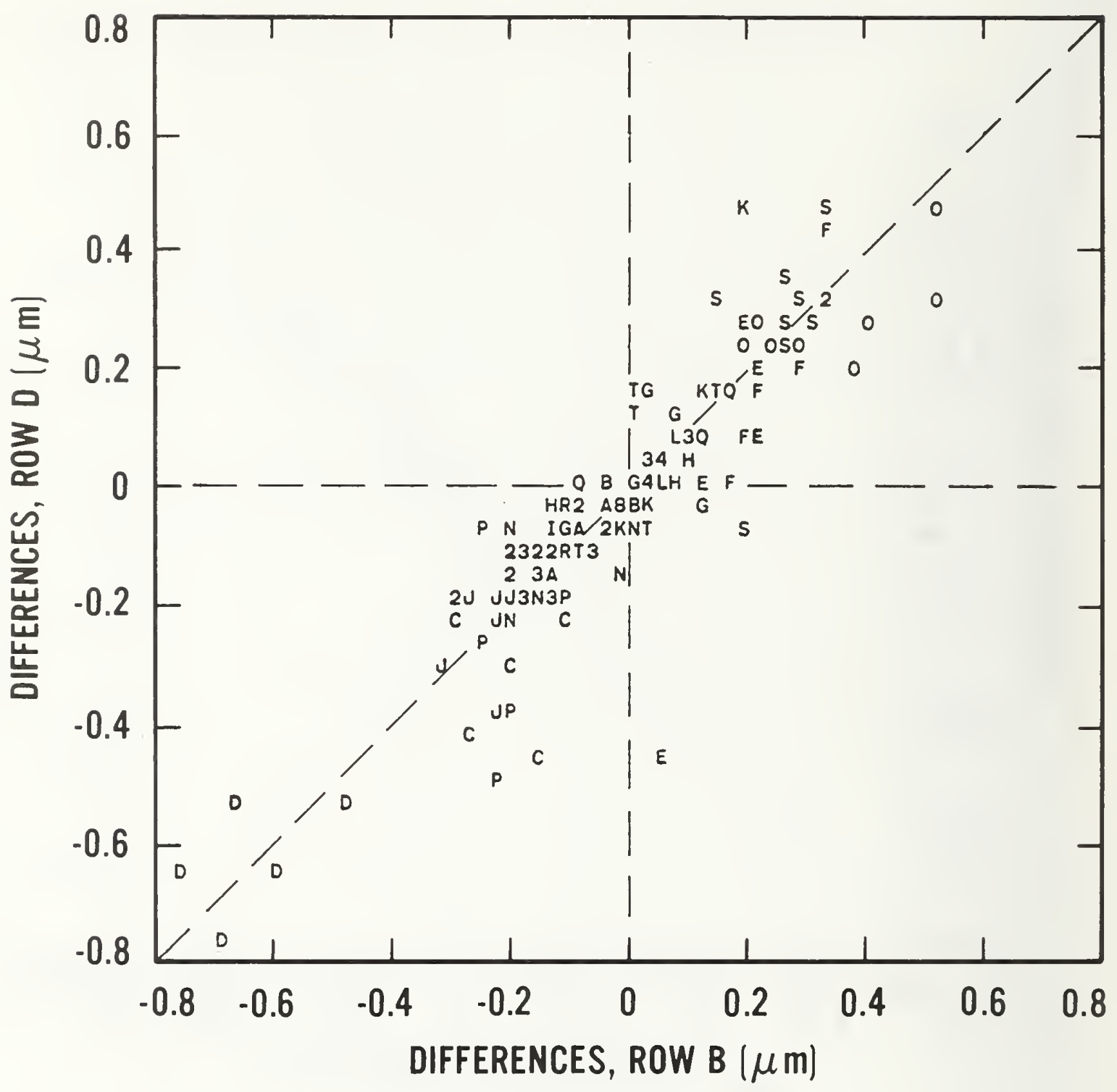

h. Lines B-0 with mean NBS value of $6.53 \mu \mathrm{m}$ and

lines D-0 with mean NBS value of $7.12 \mu \mathrm{m}$

(2 points fell outside of plotting range).

Figure 7 Continued. 


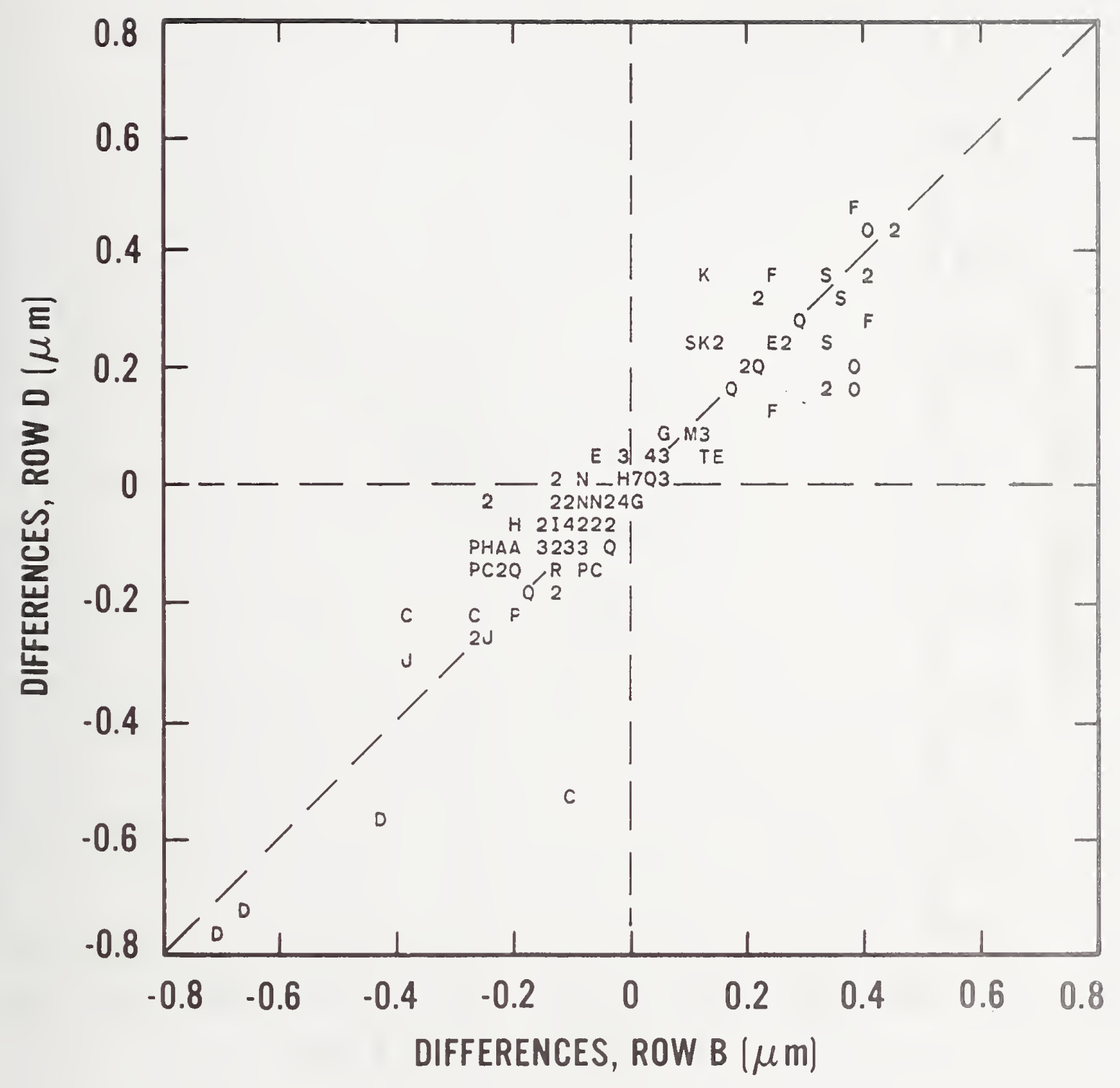

i. Lines B-2 with mean NBS value of $7.94 \mu \mathrm{m}$ and lines $D-7$ with mean NBS value of $8.67 \mu \mathrm{m}$

( 6 points fell outside of plotting range).

Figure 7 Continued. 


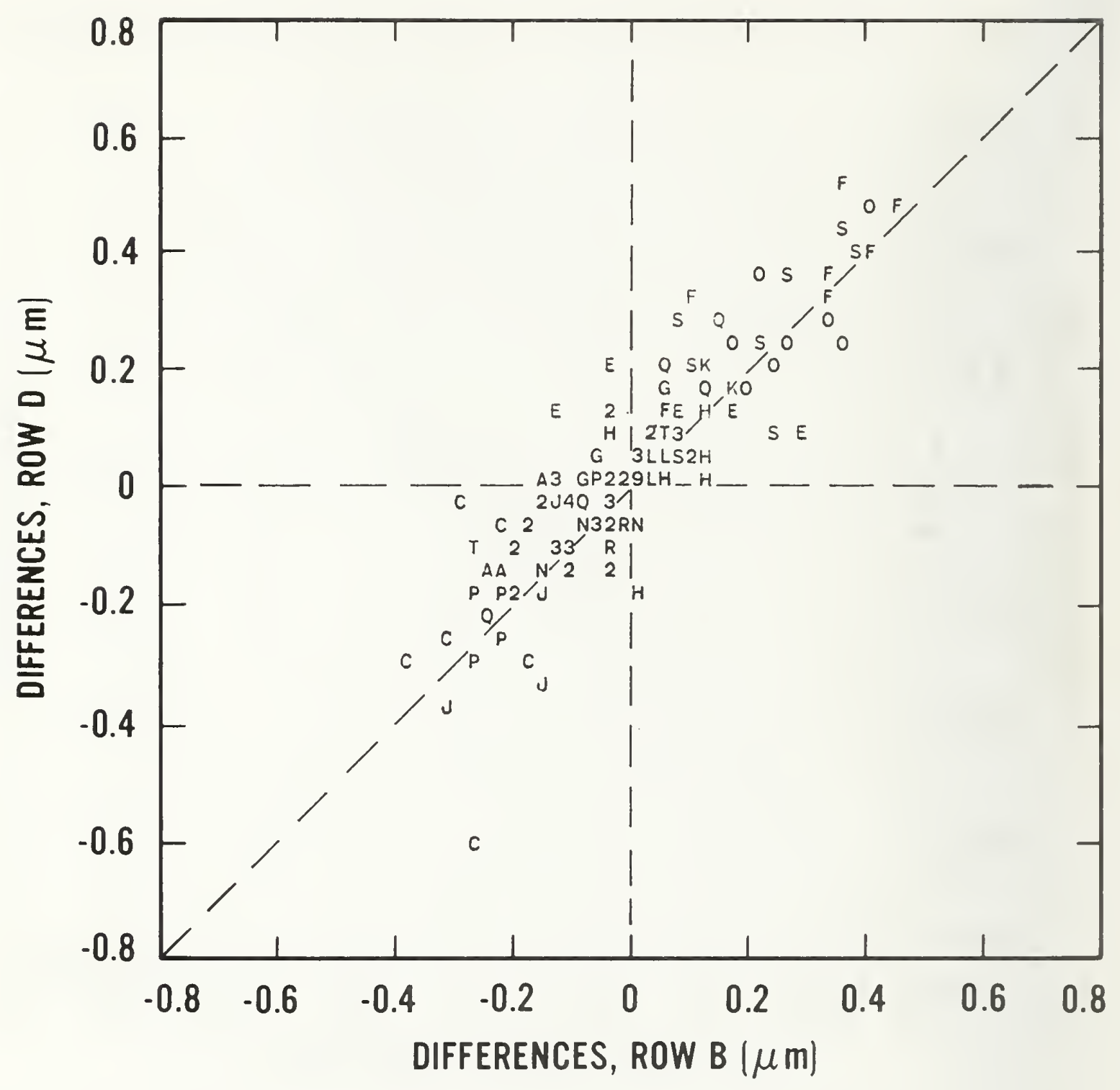

j. Lines B-5 with mean NBS value of $10.97 \mu \mathrm{m}$ and lines D-5 with mean NBS value of $9.41 \mu \mathrm{m}$ ( 8 points fell outside of plotting range).

Figure 7 Concluded. 


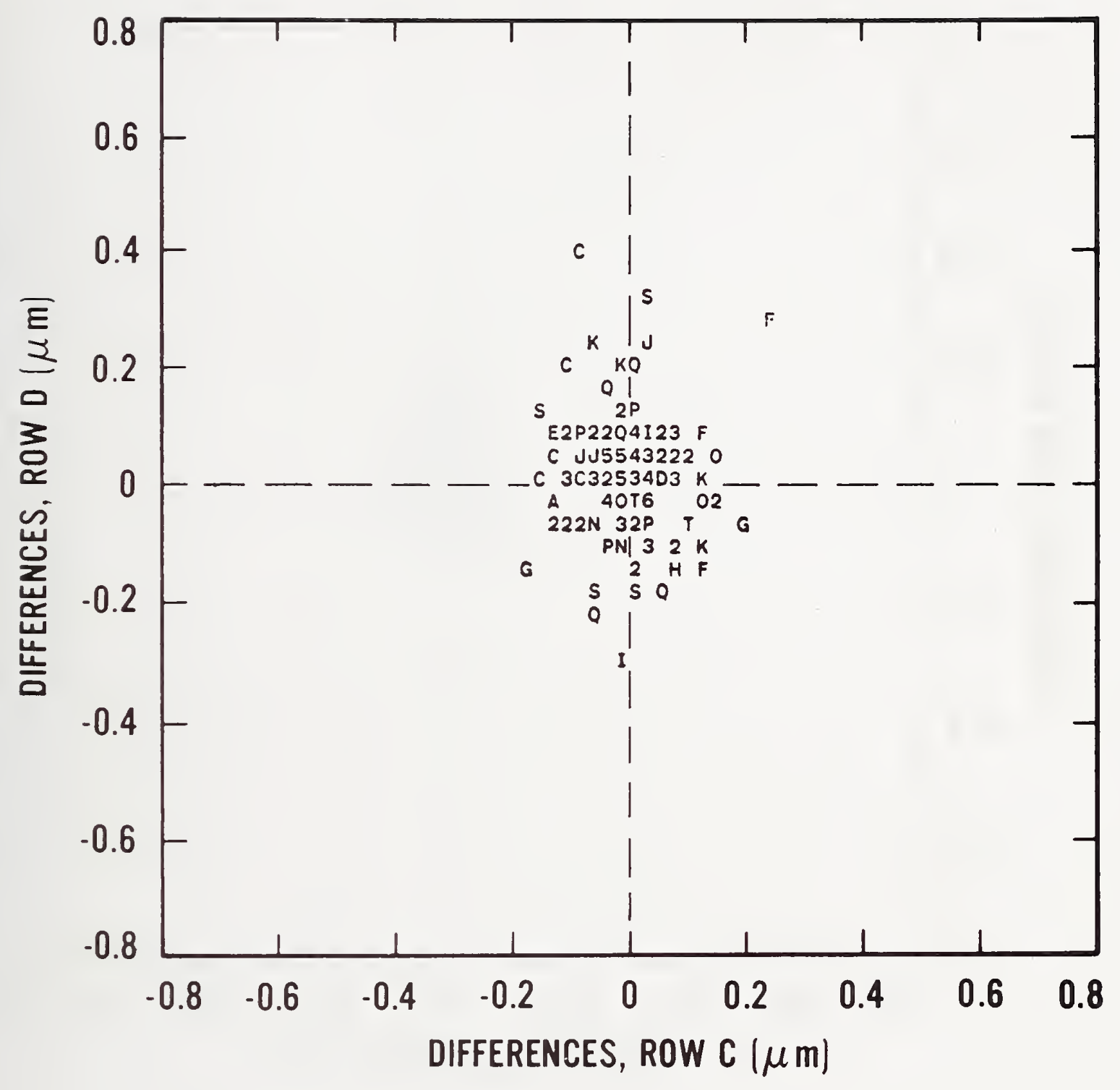

a. Lines $\mathrm{C}-3$ with mean NBS value of $0.73 \mu \mathrm{m}$ and lines $D-6$ with mean NBS value of $0.97 \mathrm{\mu m}$

Figure 8. Youden plots of differences between participants' corrected linewidth measurements on opaque and clear lines (rows $C$ and D) and NBS values. (Letters $A$ through $\mathrm{T}$ represent different measurement systems as identified in table 3.) 


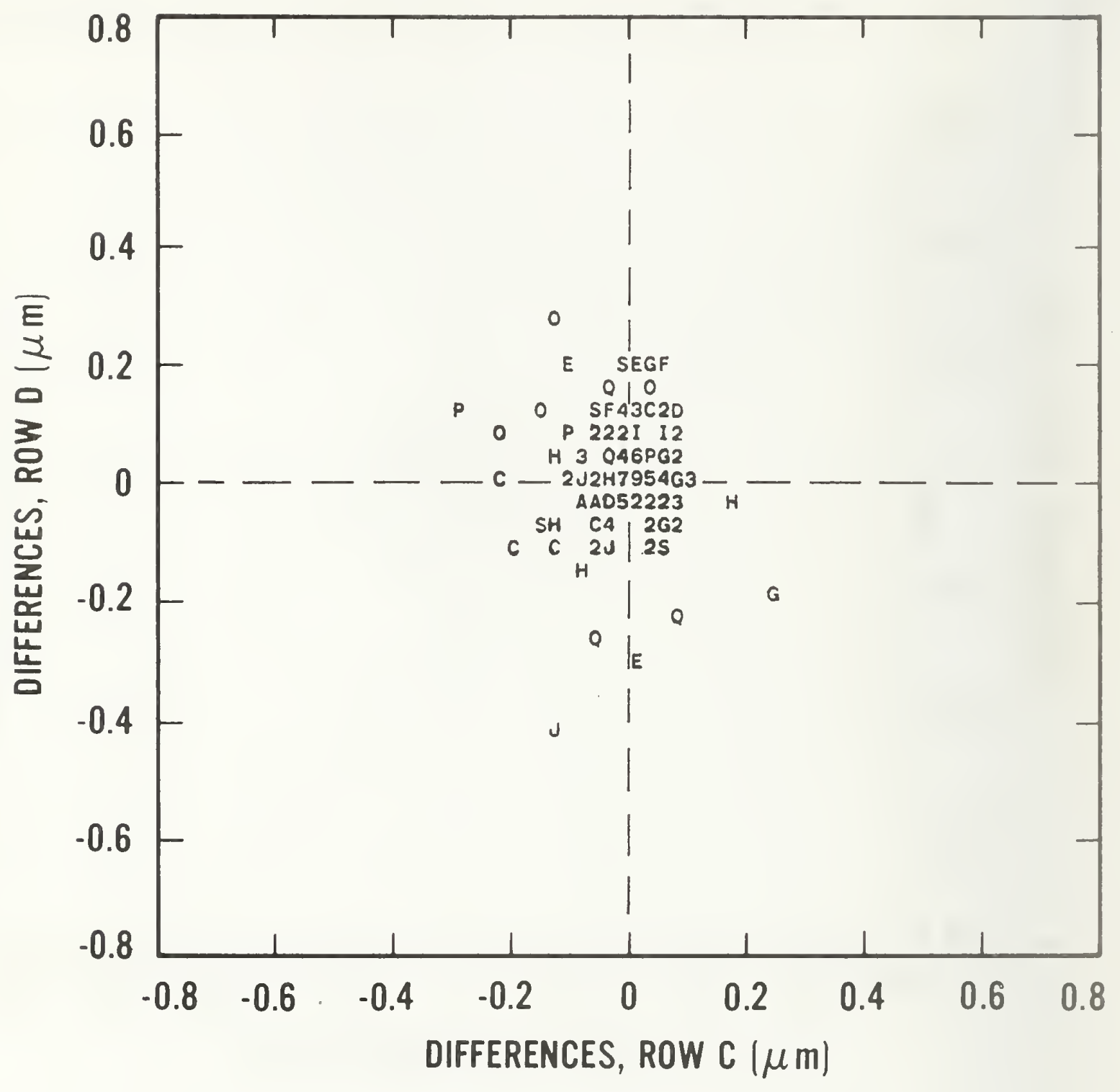

b. Lines C-6 with mean NBS value of $1.22 \mu \mathrm{m}$ and lines D-3 with mean NBS value of $1.53 \mu \mathrm{m}$.

Figure 8 Continued. 


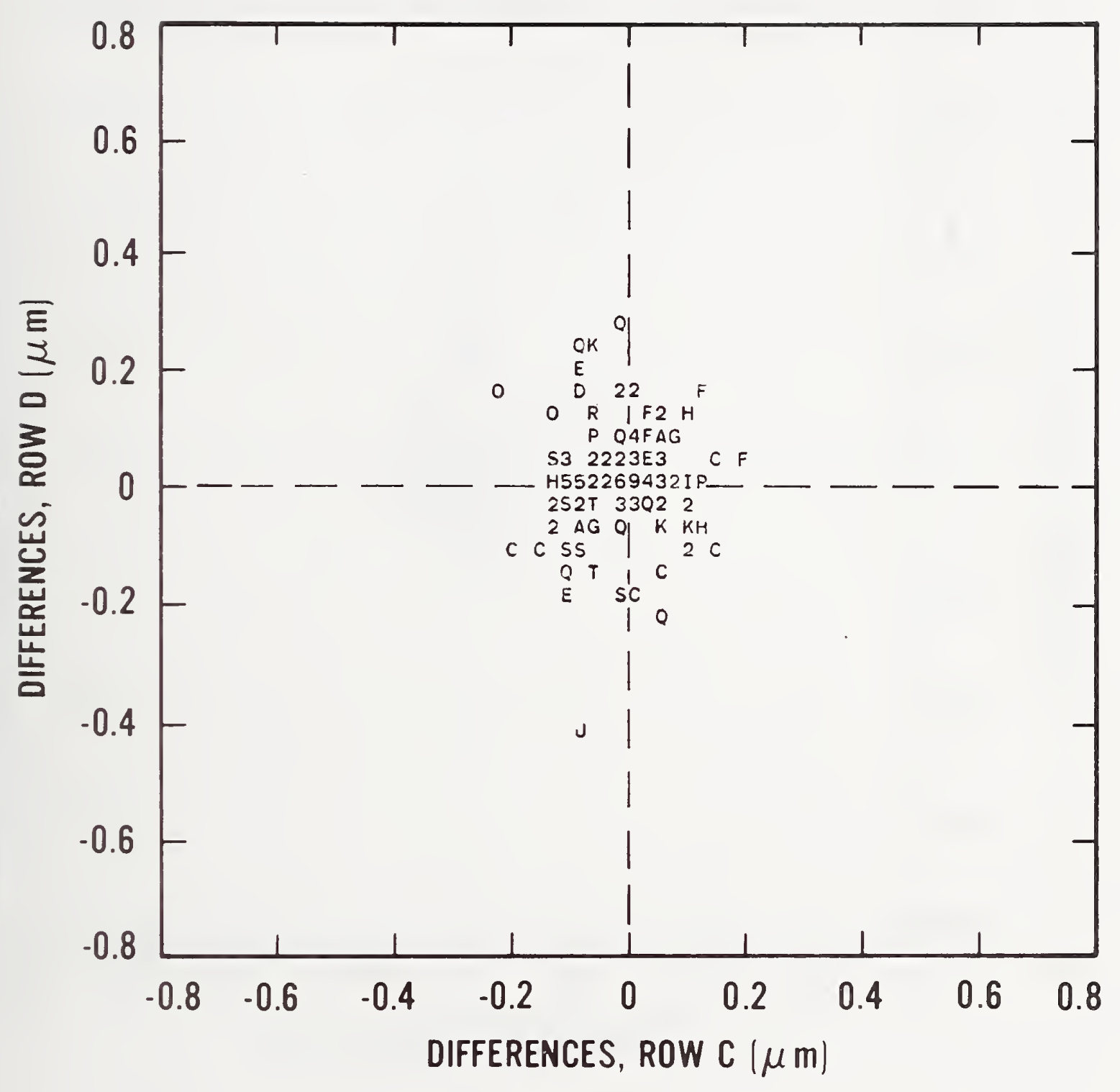

c. Lines C-1 with mean NBS value of $1.70 \mu \mathrm{m}$ and lines $D-8$ with mean NBS value of $2.10 \mu \mathrm{m}$.

Figure 8 Continued. 


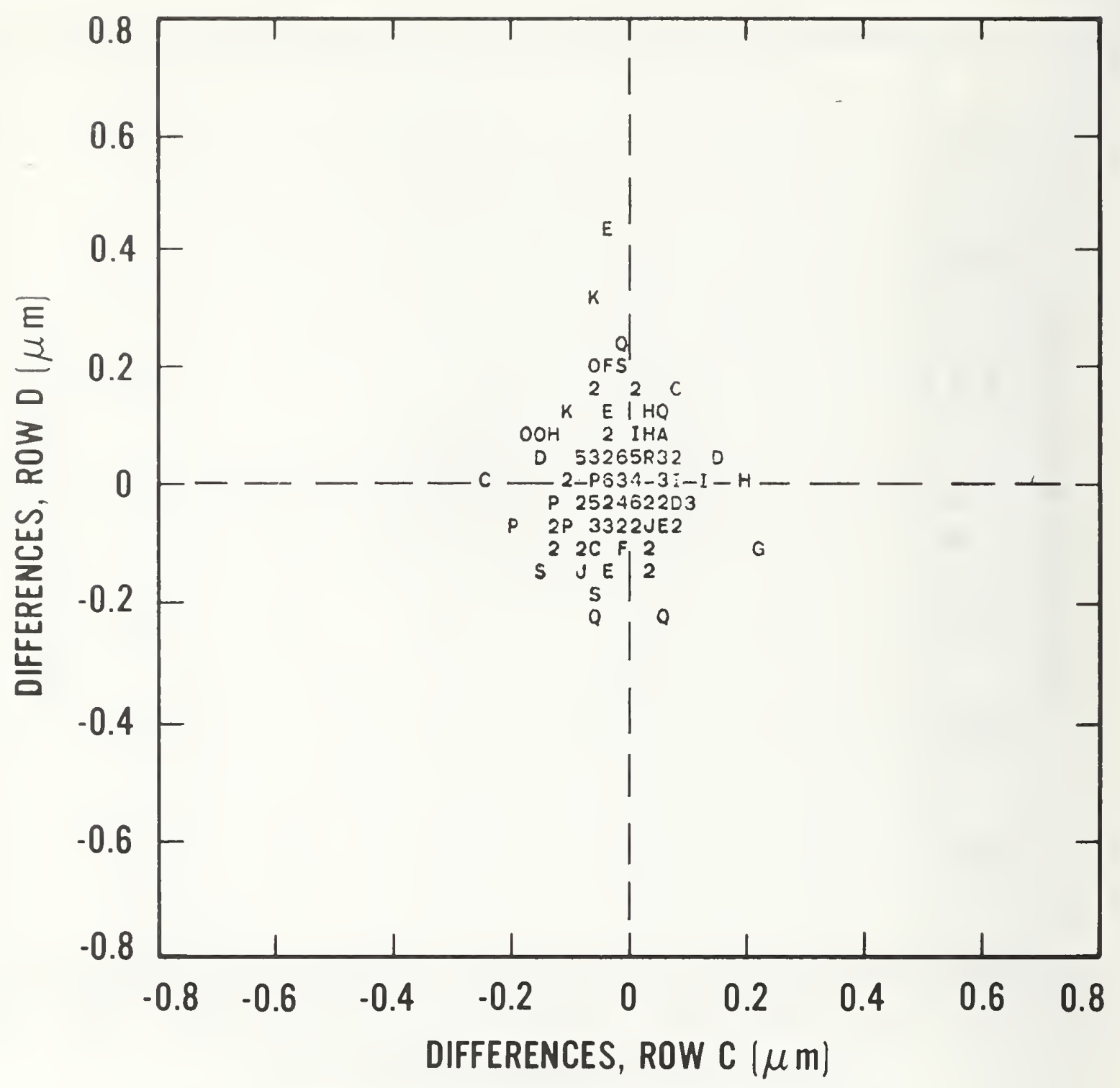

d. Lines $\mathrm{C}-8$ with mean NBS value of $2.05 \mu \mathrm{m}$ and lines $\mathrm{D}-1$ with mean NBS value of $2.40 \mu \mathrm{m}$.

Figure 8 Continued. 


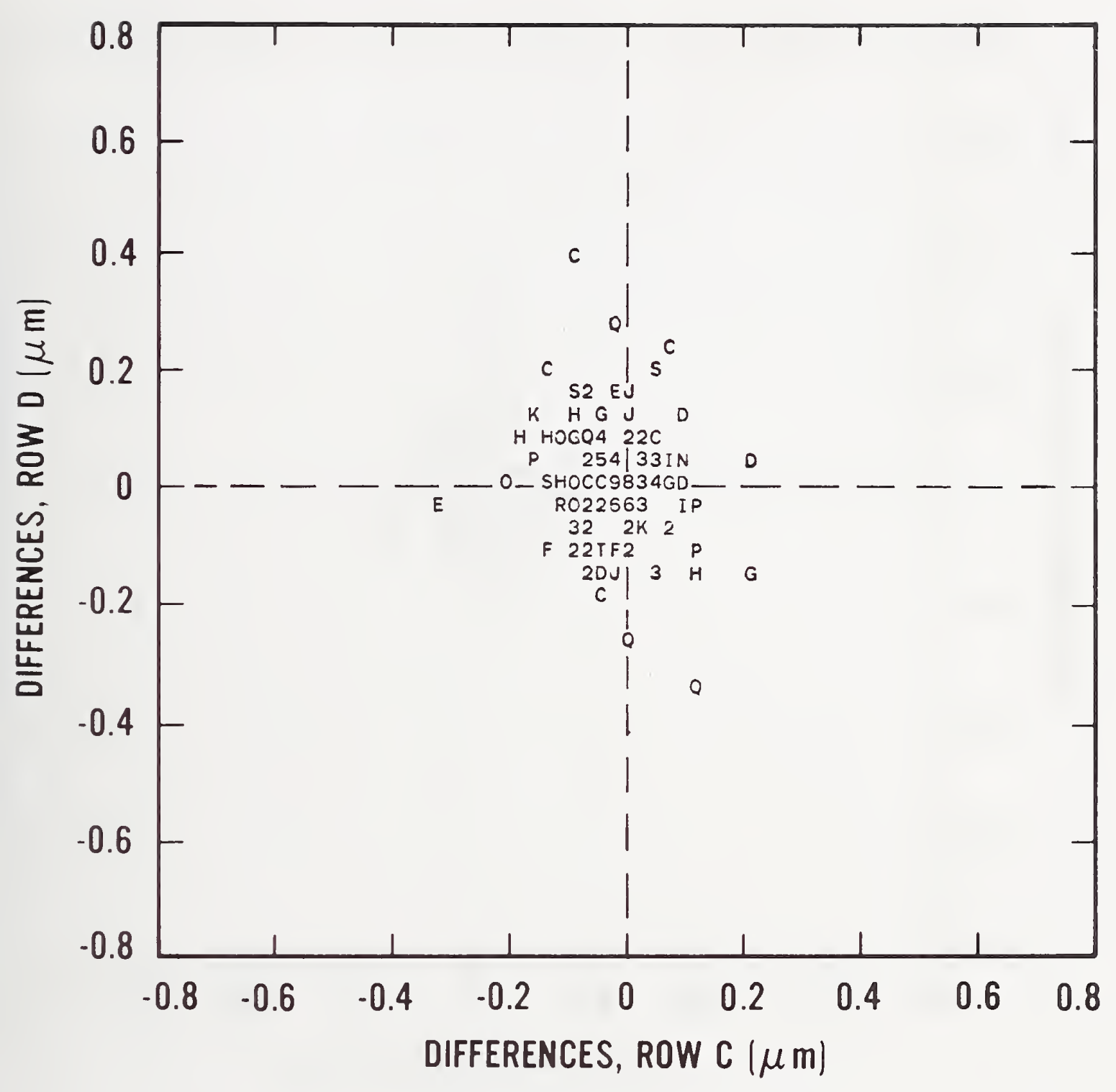

e. Lines $C-7$ with mean NBS value of $3.27 \mu \mathrm{m}$ and
lines $D-2$ with mean NBS value of $3.60 \mu \mathrm{m}$.

Figure 8 Continued. 


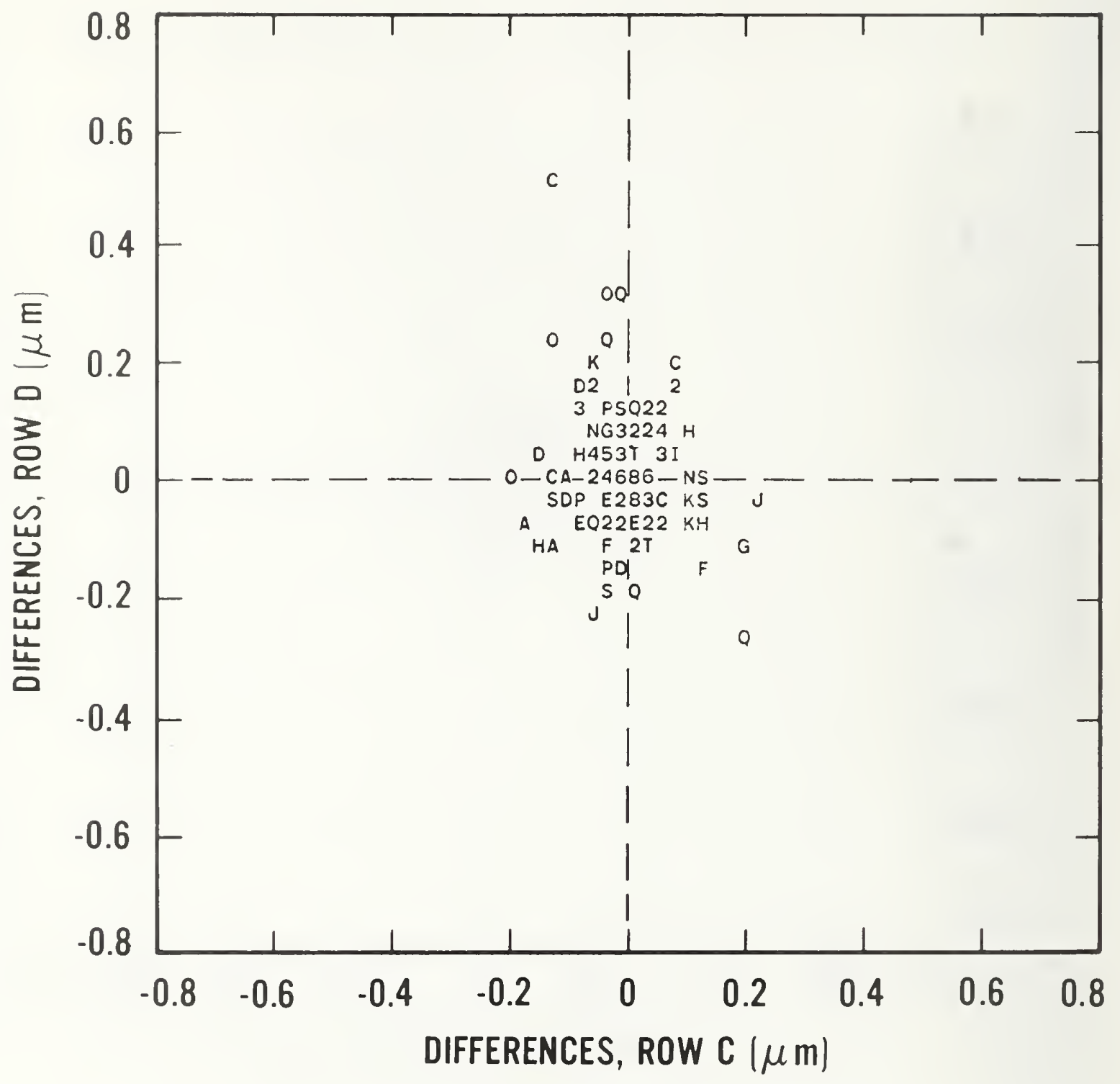

f. Lines $C-0$ with mean NBS value of $4.70 \mu \mathrm{m}$ and

lines D-9 with mean NBS value of $5.03 \mu \mathrm{m}$.

Figure 8 Continued. 


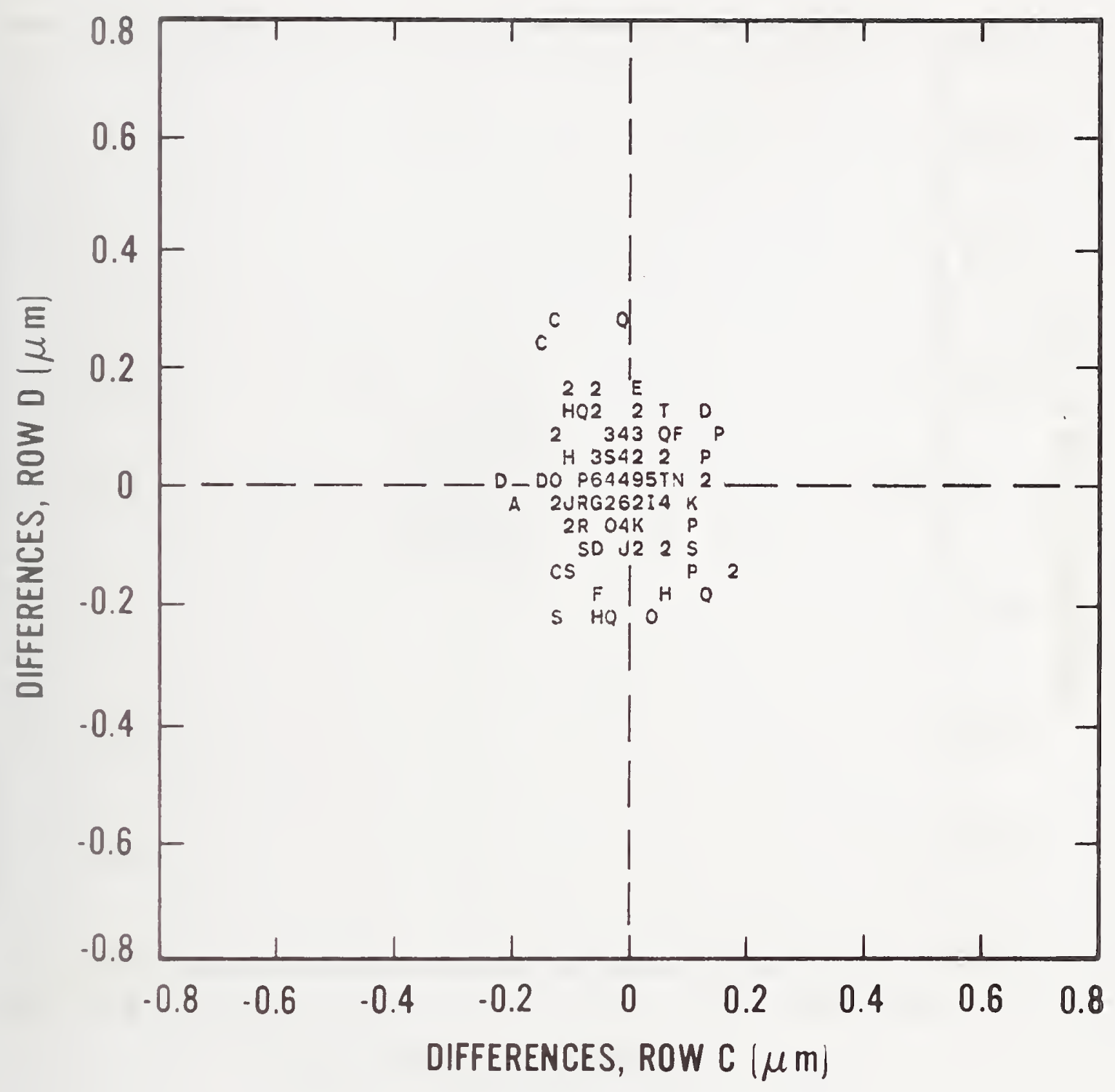

g. Lines C-5 with mean NBS value of $5.22 \mu \mathrm{m}$ and lines $D-4$ with mean NBS value of $5.67 \mu \mathrm{m}$.

Figure 8 Continued. 


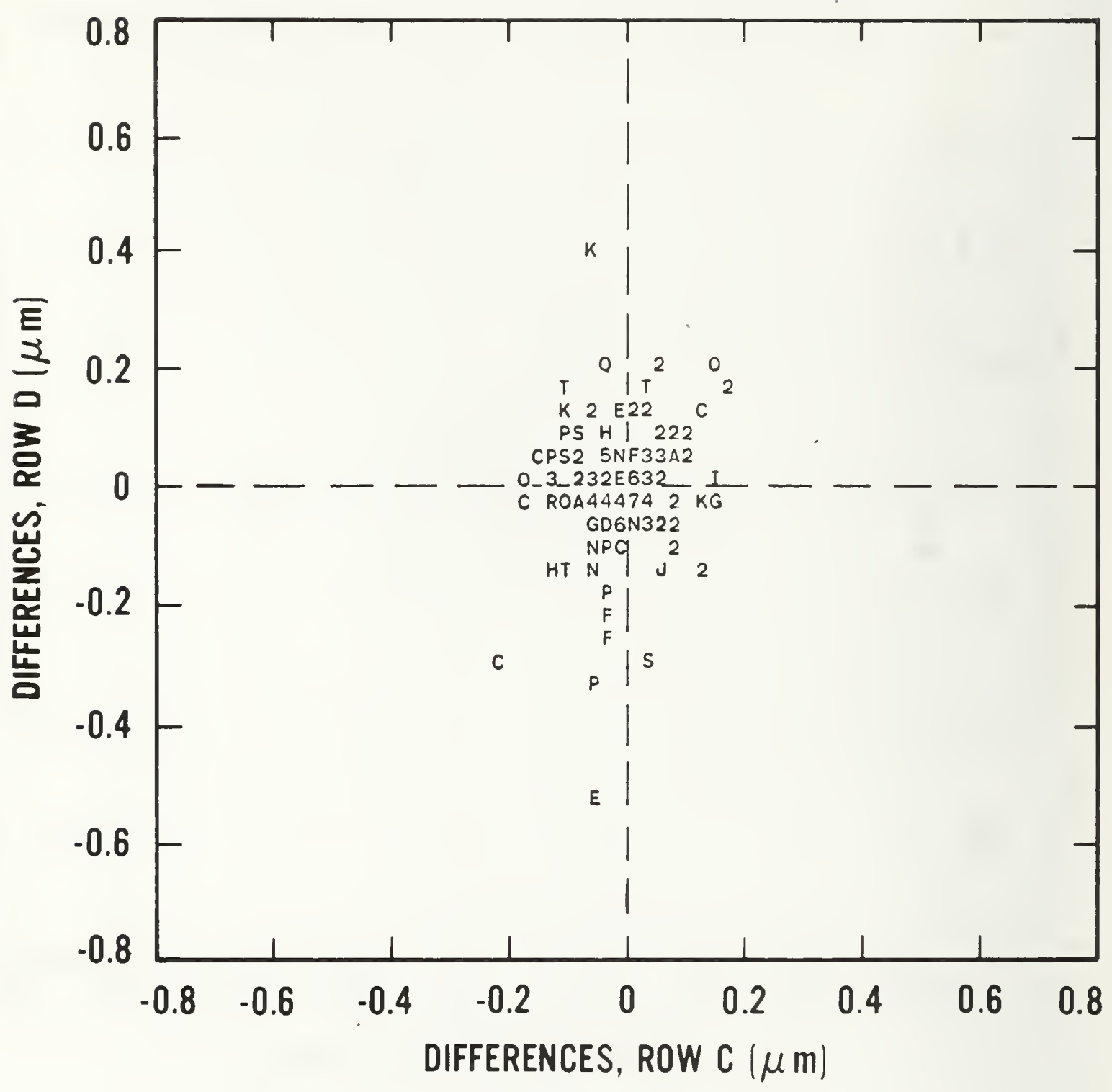

h. Lines $C-9$ with mean NBS value of $6.72 \mu \mathrm{m}$ and lines $D-0$ with mean NBS value of $7.12 \mu \mathrm{m}$.

Figure 8 Continued. 


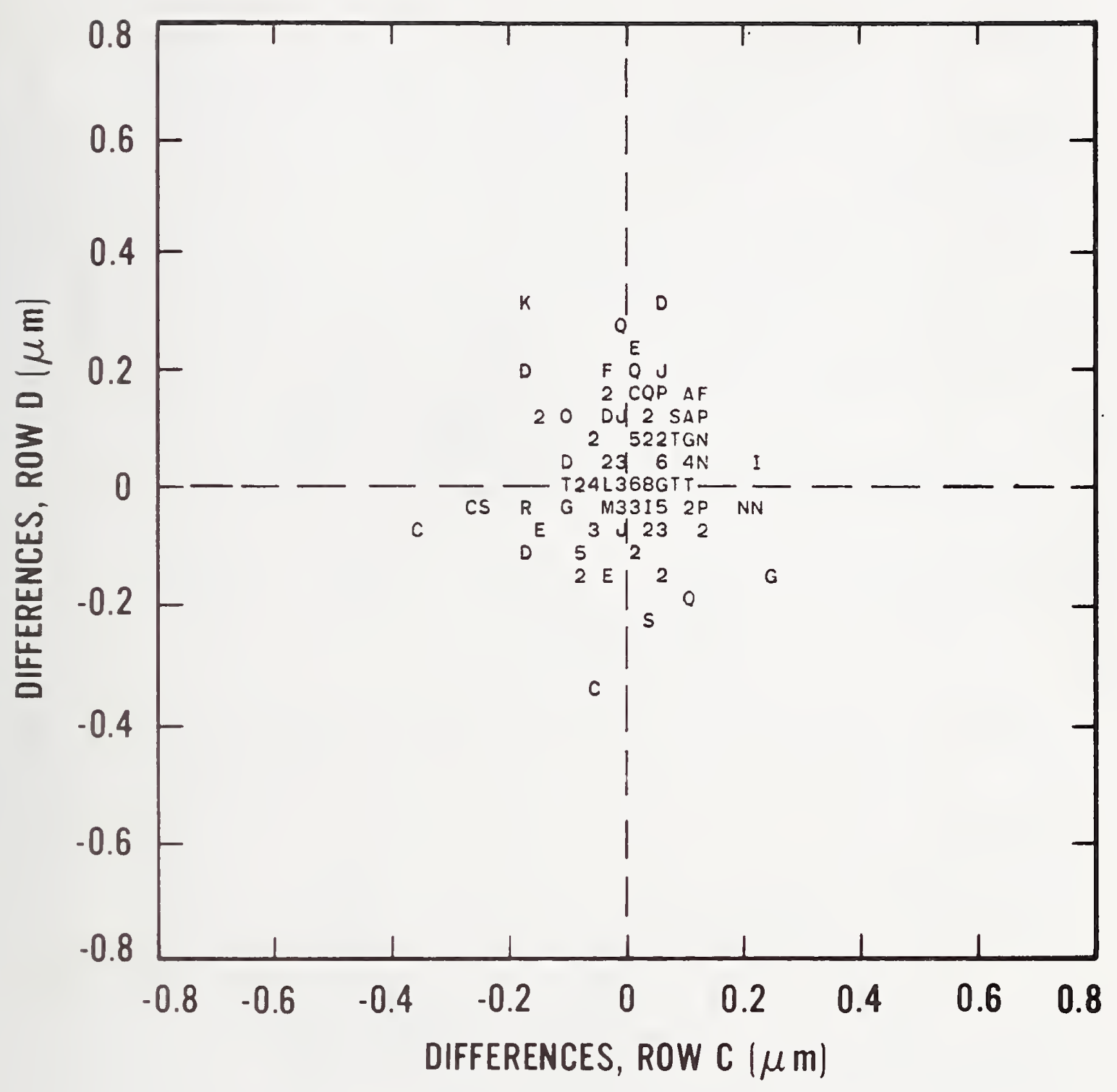

i. Lines $\mathrm{C}-2$ with mean NBS value of $8.23 \mu \mathrm{m}$ and lines $D-7$ with mean NBS value of $8.67 \mu \mathrm{m}$.

Figure 8 Continued. 


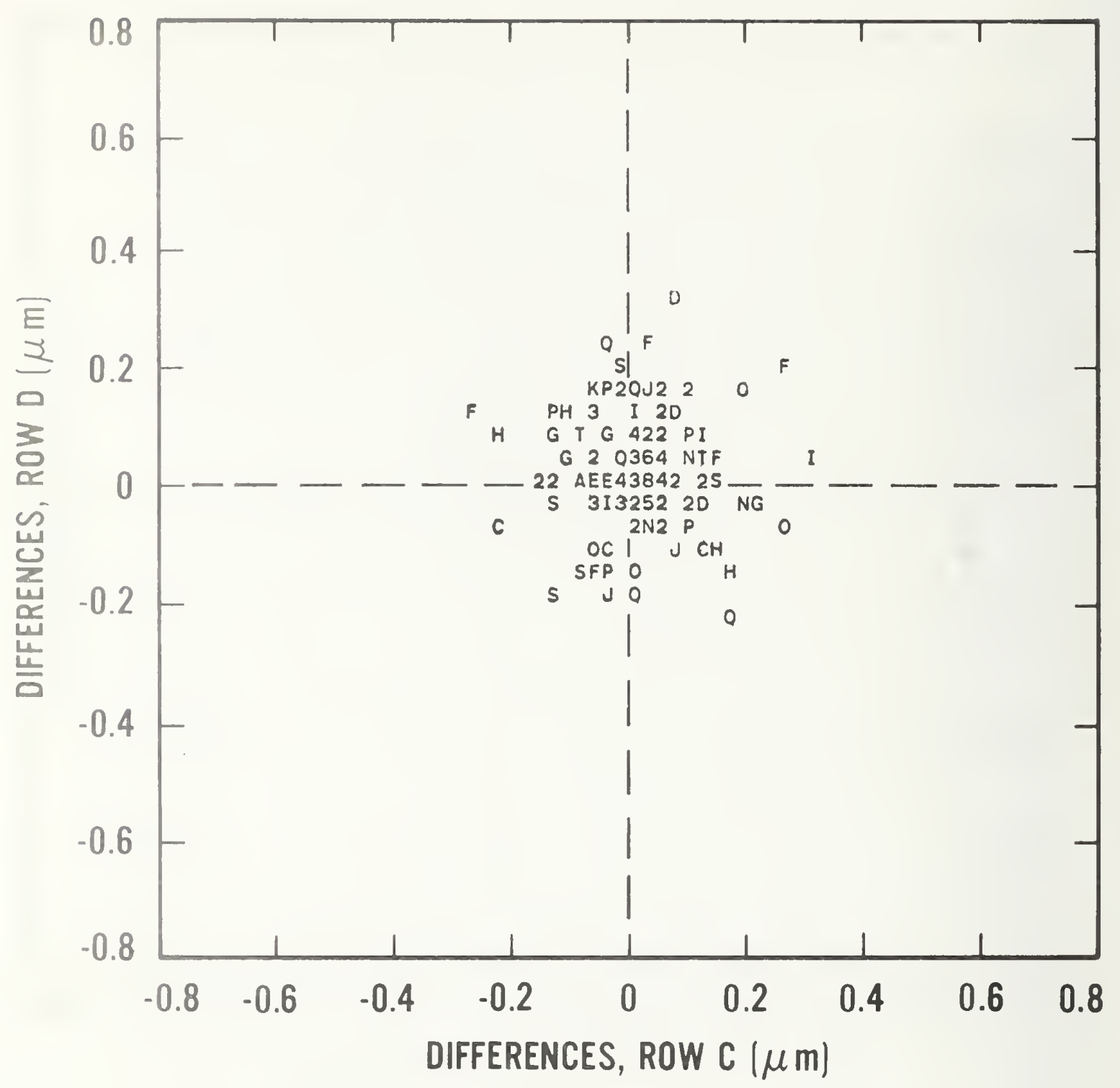

j. Lines $C-4$ with mean NBS value of $8.85 \mu \mathrm{m}$ and lines D-5 with mean NBS value of $9.41 \mu \mathrm{m}$.

Figure 8 Concluded. 


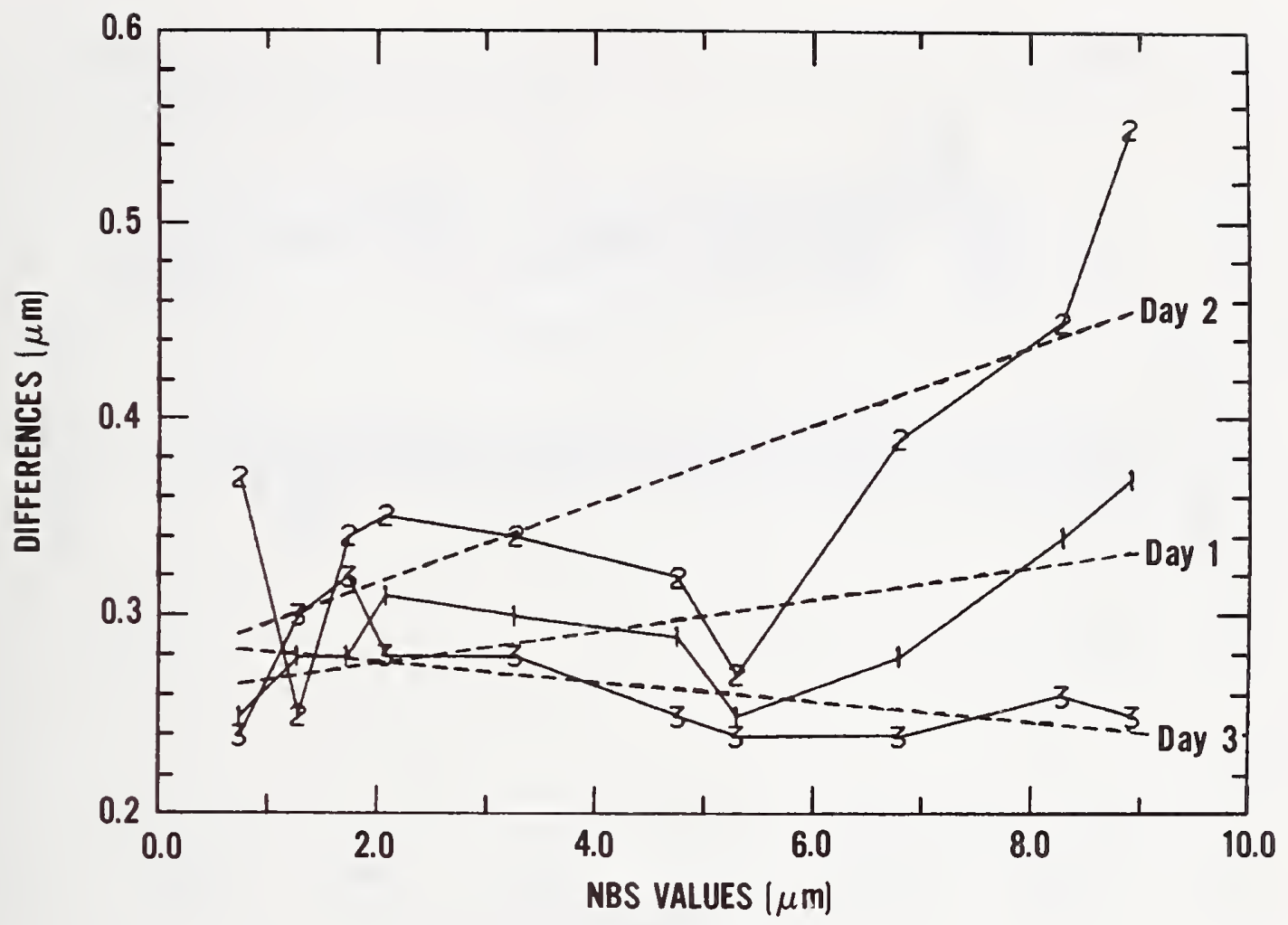

(a) Changing slope.

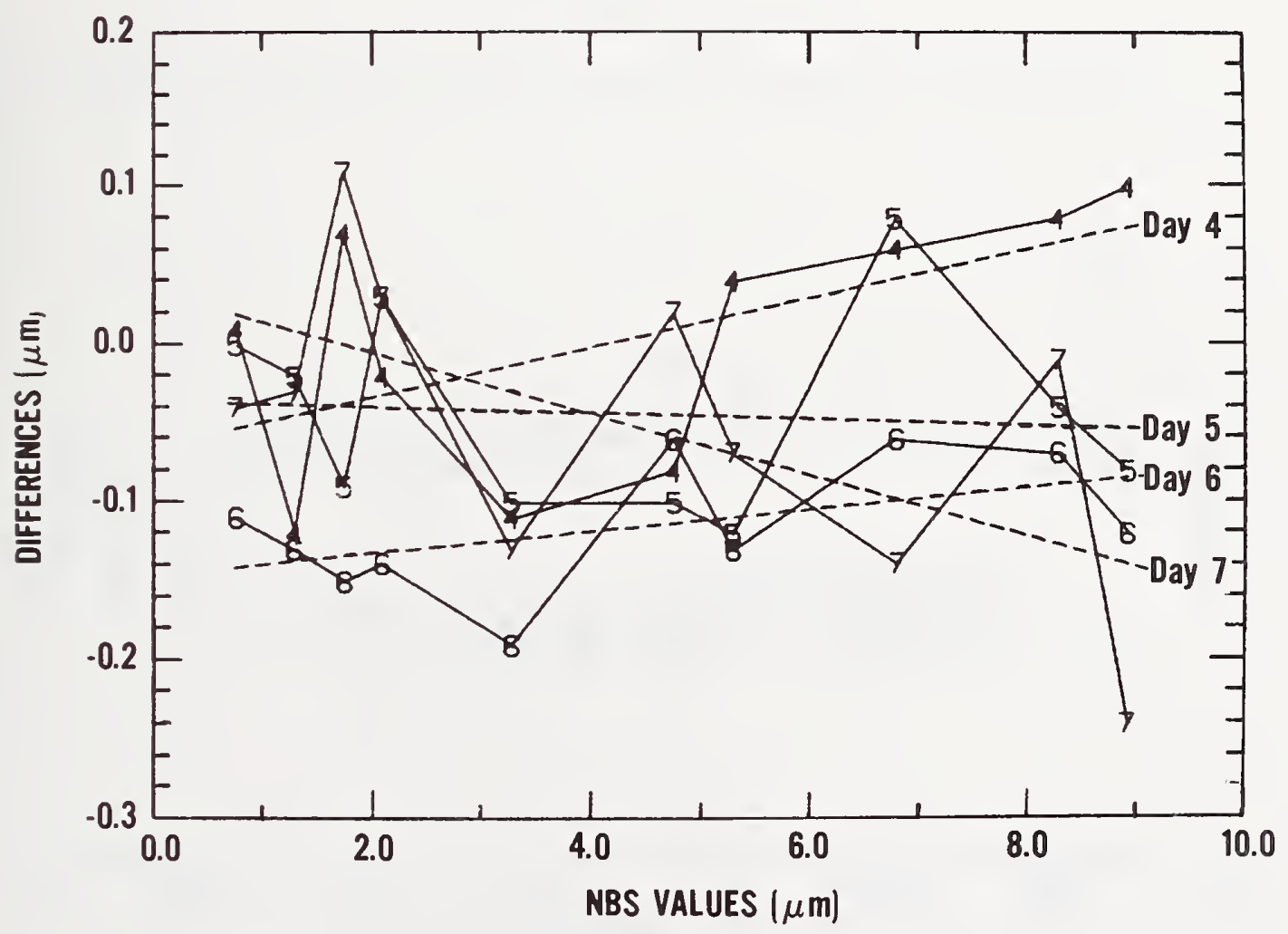

(b) Changing intercept and slope.

Figure 9. Daily changes in linear linewidth calibration curve for one operator. (Linewidth measurement differences for one day are connected by solid lines, and the corresponding linewidth calibration curve is represented by a dashed line.) 


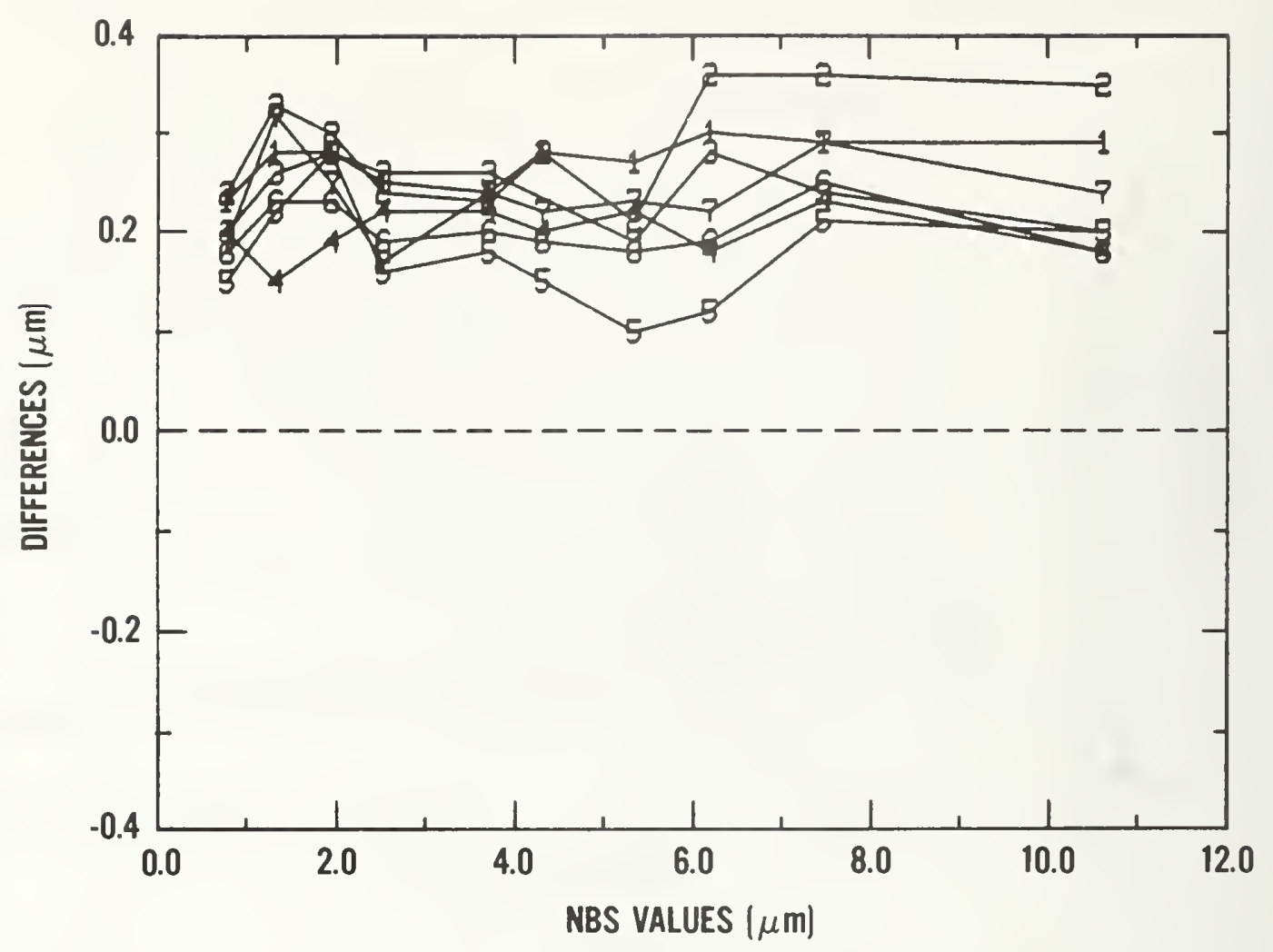

(a) Opaque lines (row A).

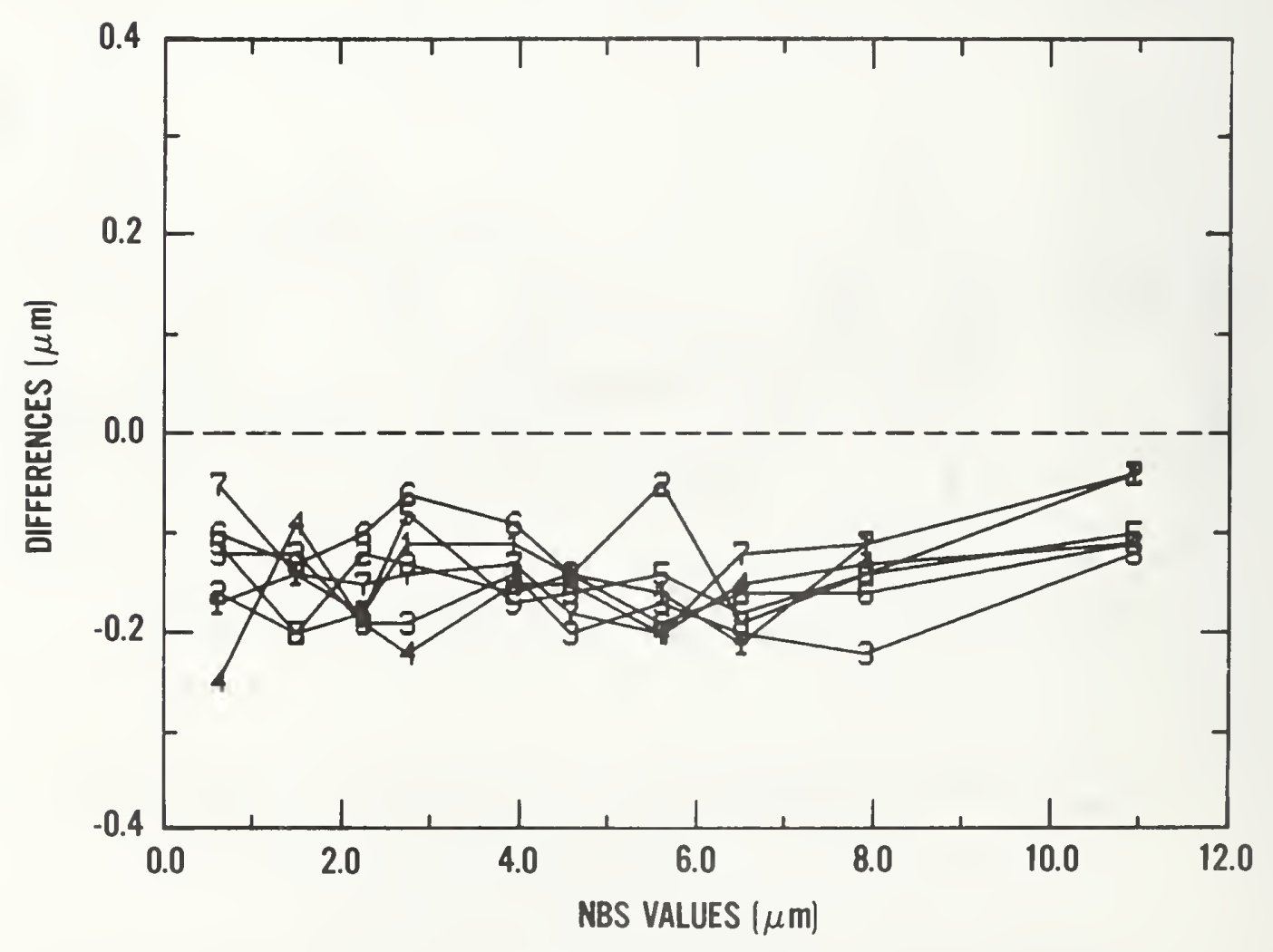

(b) Clear lines (row B).

Figure 10. Dependence of systematic linewidth measurement errors on line polarity. 


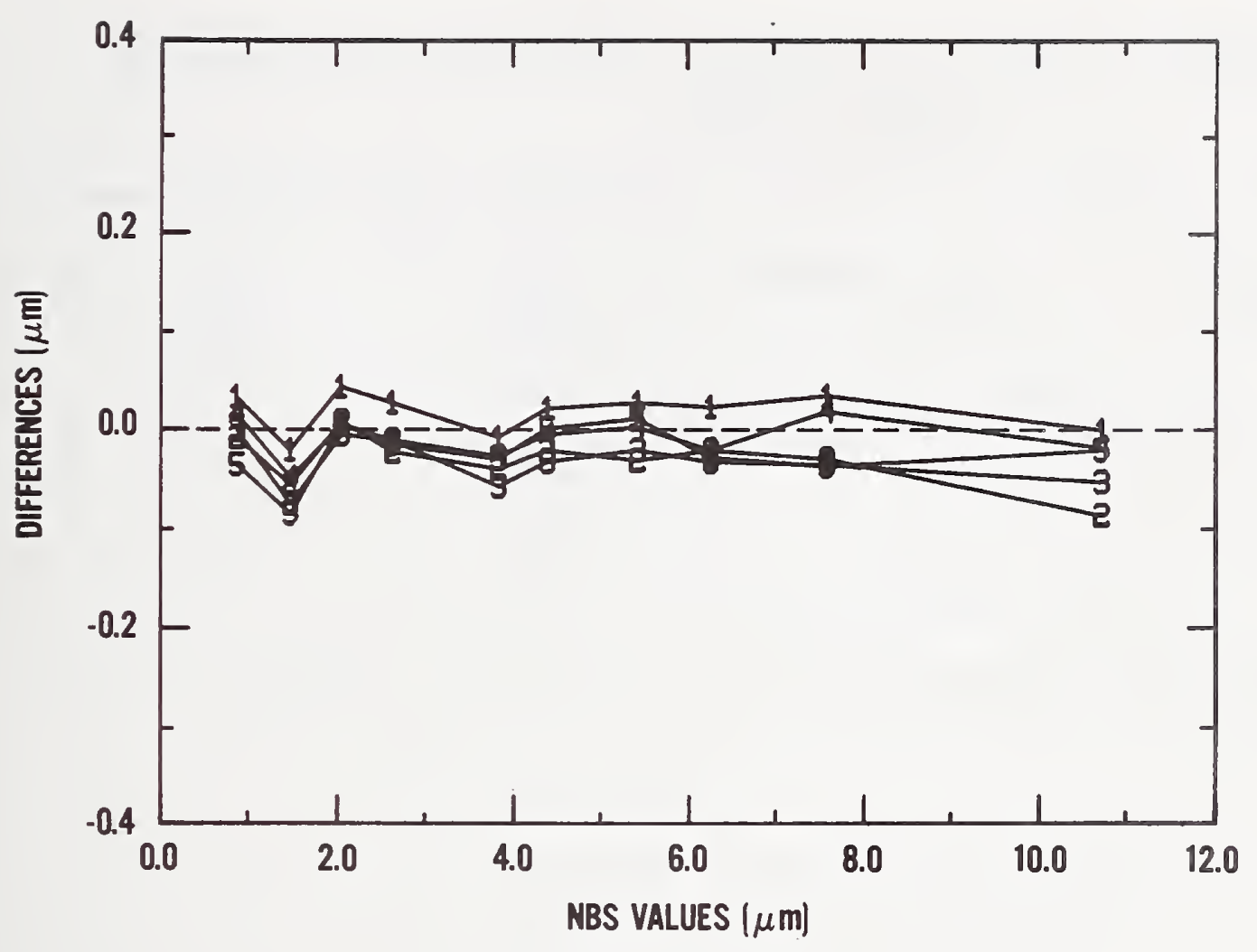

(a) Opaque lines (row A).

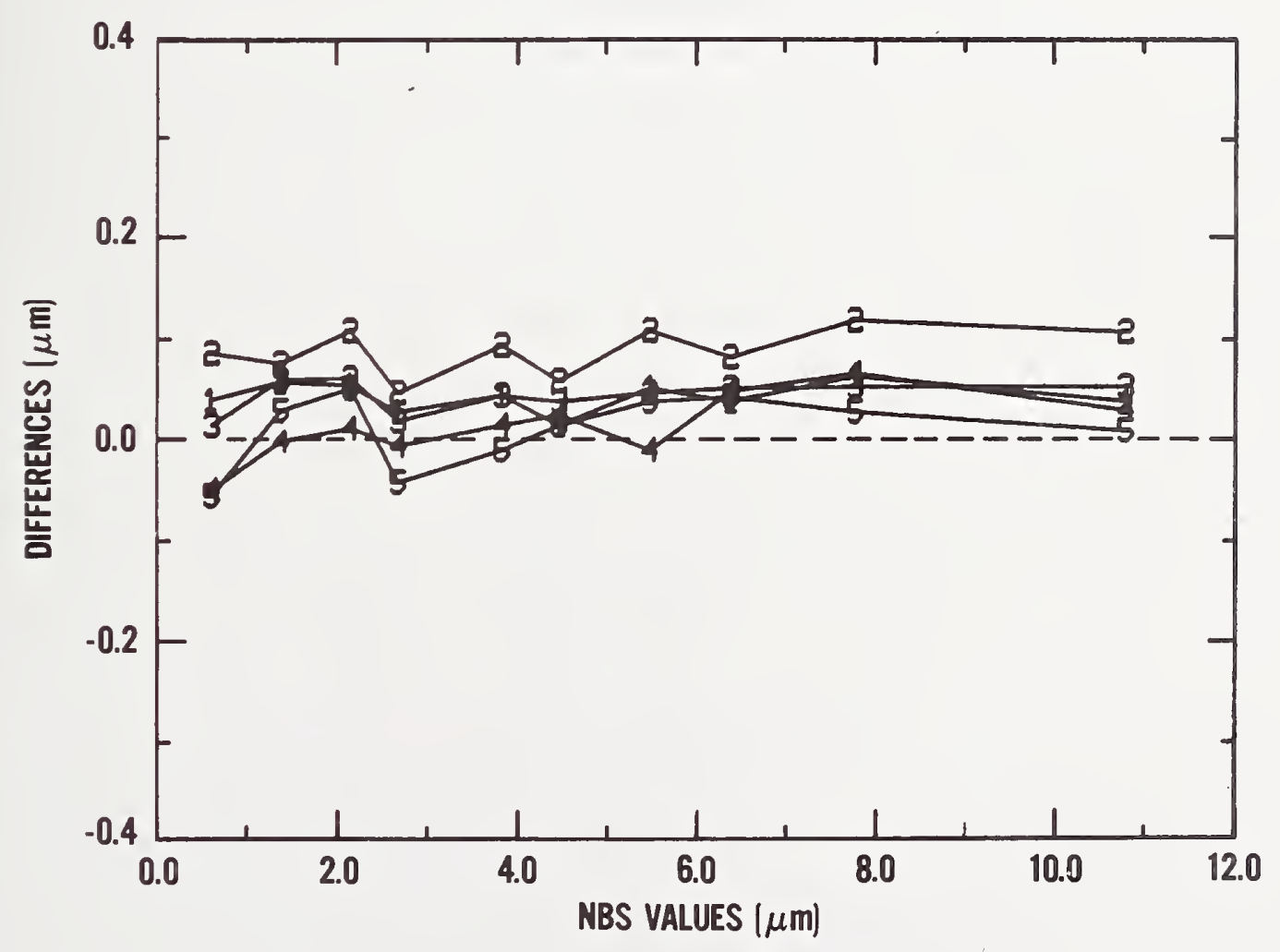

(b) Clear lines (row B).

Figure 11. Residual linewidth measurement errors of video image-scanning measurement system. 
Table 1 - Uncertainty Assigned to NBS Linewidth Measurements for Interlaboratory Study

\begin{tabular}{|c|c|c|c|c|}
\hline \multirow{3}{*}{$\begin{array}{l}\text { Artifact } \\
\text { Number }\end{array}$} & \multirow{3}{*}{$\begin{array}{l}\text { Estima ted Random } \\
\text { Component } \\
\text { (three sigma), a Im }\end{array}$} & \multicolumn{3}{|c|}{ Estimated Systematic Components, Im } \\
\hline & & \multirow{2}{*}{$\begin{array}{l}\text { Line-Edge } \\
\text { Detection }\end{array}$} & \multicolumn{2}{|c|}{$\begin{array}{l}\text { Flare and Optical- } \\
\text { Phase Correction }\end{array}$} \\
\hline & & & $\begin{array}{l}\text { Rows } \\
\mathrm{A} \text { and } \mathrm{C}\end{array}$ & $\begin{array}{l}\text { Rows } \\
B \text { and D }\end{array}$ \\
\hline $52,54-60$ & \pm 0.03 & \pm 0.05 & +0.10 & -0.08 \\
\hline 53 & \pm 0.05 & \pm 0.05 & +0.10 & -0.08 \\
\hline
\end{tabular}

a These random components are also the uncertainty associated with the line-spacing measurements on row $\mathrm{E}$. 
Table 2 - Measurement Systems Used by Participants in Interlaboratory Study

\begin{tabular}{|c|c|}
\hline Code & Measurement System \\
\hline 1 I & Image shearing with optical display \\
\hline-- & Video image scanning* \\
\hline $3 \mathrm{~V}$ & Video image scanning \\
\hline $4 I$ & Image shearing with video display \\
\hline $5 U$ & Optical filar \\
\hline 52 & Optical filar \\
\hline-- & Image shearing with optical display ${ }^{*}$ \\
\hline $6 A$ & Video filar \\
\hline $7 \mathrm{~A}$ & Video filar \\
\hline $7 \mathrm{C}$ & Video filar \\
\hline 7I & Image shearing with optical display \\
\hline $8 I$ & Image shearing with optical display \\
\hline $8 \mathrm{P}$ & Optical image scanning \\
\hline $9 \mathrm{~V}$ & Video image scanning \\
\hline $9 \mathrm{w}$ & Video image scanning \\
\hline $10 I$ & Image shearing with optical display \\
\hline $11 \mathrm{~F}$ & Optical filar \\
\hline $11 \mathrm{I}$ & Image shearing with optical display \\
\hline-- & Optical image scanning* \\
\hline $13 \mathrm{C}$ & Video filar \\
\hline $13 I$ & Image shearing with optical display \\
\hline $14 \mathrm{C}$ & Video filar \\
\hline $15 B$ & Video filar \\
\hline-- & Video image scanning* \\
\hline
\end{tabular}

* Data were not analyzed by NBS. 
Table 3 - Codes for Identifying Participants' Measurement Systems in Figures 6, 7, and 8

\begin{tabular}{|c|c|c|c|}
\hline (See & $\begin{array}{l}\text { Code } \\
\text { figs. } 6,7 \text {, and 8.) }\end{array}$ & $\begin{array}{l}\text { Code } \\
\text { (See table 2.) }\end{array}$ & Mea surement System \\
\hline & $A$ & $1 \mathrm{I}$ & $\begin{array}{l}\text { Image shearing with optical } \\
\text { display }\end{array}$ \\
\hline & B & $3 \mathrm{~V}$ & Video image scanning \\
\hline & $\mathrm{C}$ & $4 I$ & $\begin{array}{l}\text { Image shearing with video } \\
\text { display }\end{array}$ \\
\hline & $\mathrm{D}$ & $5 U$ & Optical filar \\
\hline & $\mathrm{E}$ & $5 z$ & Optical filar \\
\hline & $\mathrm{F}$ & $6 \mathrm{~A}$ & Video filar \\
\hline & G & $7 A$ & Video filar \\
\hline & $\mathrm{H}$ & $7 \mathrm{C}$ & Video filar \\
\hline & I & $7 I$ & $\begin{array}{l}\text { Image shearing with optical } \\
\text { display }\end{array}$ \\
\hline & $\mathrm{J}$ & $8 I$ & $\begin{array}{l}\text { Image shearing with optical } \\
\text { display }\end{array}$ \\
\hline & K & $8 \mathrm{P}$ & Optical image scanning \\
\hline & L & $9 \mathrm{~V}$ & Video image scanning \\
\hline & M & $9 \mathrm{~W}$ & Video image scanning \\
\hline & $\mathrm{N}$ & $10 \mathrm{I}$ & $\begin{array}{l}\text { Image shearing with optical } \\
\text { display }\end{array}$ \\
\hline & 0 & $11 \mathrm{~F}$ & Optical filar \\
\hline & $\mathrm{P}$ & $11 \mathrm{I}$ & $\begin{array}{l}\text { Image shearing with optical } \\
\text { display }\end{array}$ \\
\hline & Q & $13 \mathrm{C}$ & Video filar \\
\hline & $\mathrm{R}$ & $13 I$ & $\begin{array}{l}\text { Image shearing with optical } \\
\text { display }\end{array}$ \\
\hline & s & $14 \mathrm{C}$ & Video filar \\
\hline & $\mathrm{T}$ & $15 B$ & Video filar \\
\hline
\end{tabular}


Table 4 - Least-Squares Estimates of Intercept $\alpha$ and Slope $\beta$ for Linespacing Calibration Curves and Student's $t$ Statistics

\begin{tabular}{|c|c|c|c|c|}
\hline $\begin{array}{l}\text { Measurement } \\
\text { System }\end{array}$ & $\hat{\alpha}$ & $t_{1}$ & $\hat{\beta}$ & $t_{2}$ \\
\hline $1 I$ & -0.034 & -1.5 & 0.995 & 1.7 \\
\hline $3 V$ & -0.009 & -1.3 & 1.001 & -1.0 \\
\hline $4 I$ & 0.007 & 1.8 & 0.999 & 0.2 \\
\hline $5 U^{+}$ & 0.035 & 1.4 & 0.882 & $29.5 *$ \\
\hline $5 z^{\dagger}$ & 0.061 & $5.1 *$ & 0.994 & $3.0 *$ \\
\hline $6 A^{\dagger}$ & 0.030 & $6.0 *$ & 0.996 & $4.0 *$ \\
\hline $7 \mathrm{~A}$ & 0.017 & 2.1 & 0.998 & 2.0 \\
\hline $7 C^{+}$ & -0.094 & $-5.9 *$ & 1.011 & $-5.5 *$ \\
\hline $7 I$ & 0.030 & 2.1 & 1.004 & -2.0 \\
\hline $8 I$ & 0.025 & 0.6 & 0.996 & 0.7 \\
\hline $8 P$ & -0.007 & -0.5 & 1.001 & -0.5 \\
\hline $9 \mathrm{~V}$ & -0.008 & $-1 \cdot 3$ & 1.001 & -1.0 \\
\hline $9 w$ & -0.008 & -1.6 & 1.002 & -2.0 \\
\hline $10 I$ & -0.019 & -1.5 & 1.003 & -1.5 \\
\hline $11 \mathrm{~F}$ & 0.021 & 0.9 & 1.003 & -1.0 \\
\hline $11 \mathrm{I}$ & 0.024 & 1.0 & 1.004 & $-1 \cdot 3$ \\
\hline $13 C^{\dagger}$ & -0.133 & $-8.9 *$ & 1.014 & $-7.0 *$ \\
\hline $13 I$ & 0.013 & 0.8 & 0.997 & 1.5 \\
\hline $14 \mathrm{C}^{\dagger}$ & -0.053 & $-3.8 *$ & 1.004 & -2.0 \\
\hline $15 \mathrm{~B}^{\dagger}$ & 0.243 & $12.8^{*}$ & 0.986 & 4. 7* \\
\hline
\end{tabular}

* Exceeds critical value for a two-sided test at the 0.95 probability level.

+ Needs line-spacing correction to linewidth data. 
Table 5 - Least-Square Estimates of Intercept a and Slope $b$ of Linewidth Calibration Curves and Student's t Statistics for Opaque Lines (Row A) and Clear Lines (Row B)

\begin{tabular}{|c|c|c|c|c|c|}
\hline $\begin{array}{l}\text { Measurement } \\
\text { System }\end{array}$ & Row & $\hat{\alpha}$ & $t_{1}$ & $\hat{\beta}$ & $t_{2}$ \\
\hline II & $\begin{array}{l}\mathrm{A} \\
\mathrm{B}\end{array}$ & $\begin{array}{r}0.207 \\
-0.010\end{array}$ & $\begin{array}{l}10.3^{\star} \\
-0.6\end{array}$ & $\begin{array}{l}0.993 \\
0.984\end{array}$ & $\begin{array}{l}1.7 \\
5.3 *\end{array}$ \\
\hline $3 V$ & $\begin{array}{l}\mathrm{A} \\
\mathrm{B}\end{array}$ & $\begin{array}{r}0.000 \\
-0.054\end{array}$ & $\begin{array}{c}0.0 \\
-7.7 \star\end{array}$ & $\begin{array}{l}0.999 \\
1.006\end{array}$ & $\begin{array}{c}1.0 \\
-6.0\end{array}$ \\
\hline $4 I$ & $\begin{array}{l}\text { A } \\
\text { B }\end{array}$ & $\begin{array}{r}0.175 \\
-0.128\end{array}$ & $\begin{array}{r}6.2^{\star} \\
-4.6^{\star}\end{array}$ & $\begin{array}{l}1.002 \\
0.992\end{array}$ & $\begin{array}{r}-0.4 \\
1.6\end{array}$ \\
\hline $5 U$ & $\begin{array}{l}\mathrm{A} \\
\mathrm{B}\end{array}$ & $\begin{array}{r}-0.129 \\
0.168\end{array}$ & $\begin{array}{r}-5.6^{\star} \\
5.1^{\star}\end{array}$ & $\begin{array}{l}1.001 \\
0.994\end{array}$ & $\begin{array}{r}-0.2 \\
1.0\end{array}$ \\
\hline $5 z$ & $\begin{array}{l}\text { A } \\
\text { B }\end{array}$ & $\begin{array}{r}-0.081 \\
0.016\end{array}$ & $\begin{array}{c}-2.5^{\star} \\
0.9\end{array}$ & $\begin{array}{l}1.009 \\
1.008\end{array}$ & $\begin{array}{l}-1.5 \\
-2.7\end{array}$ \\
\hline $6 A$ & $\begin{array}{l}\mathrm{A} \\
\mathrm{B}\end{array}$ & $\begin{array}{l}0.182 \\
0.330\end{array}$ & $\begin{array}{r}5.5^{\star} \\
17.4^{\star}\end{array}$ & $\begin{array}{l}0.974 \\
0.996\end{array}$ & $\begin{array}{l}4.3^{\star} \\
1.3^{2}\end{array}$ \\
\hline $7 A$ & $\begin{array}{l}A \\
B\end{array}$ & $\begin{array}{l}0.108 \\
0.090\end{array}$ & $\begin{array}{r}9.0 * \\
12.9 *\end{array}$ & $\begin{array}{l}0.990 \\
0.992\end{array}$ & $\begin{array}{l}5.0 * \\
8.0^{*}\end{array}$ \\
\hline $7 \mathrm{C}$ & $\begin{array}{l}\mathrm{A} \\
\mathrm{B}\end{array}$ & $\begin{array}{l}0.092 \\
0.017\end{array}$ & $\begin{array}{l}5.7^{\star} \\
1.2\end{array}$ & $\begin{array}{l}0.987 \\
0.998\end{array}$ & $\begin{array}{l}4 \cdot 3^{\star} \\
1.3\end{array}$ \\
\hline $7 I$ & $\begin{array}{l}\mathrm{A} \\
\mathrm{B}\end{array}$ & $\begin{array}{r}0.224 \\
-0.159\end{array}$ & $\begin{array}{r}16.0^{\star} \\
-11.4^{*}\end{array}$ & $\begin{array}{l}1.001 \\
1.004\end{array}$ & $\begin{array}{l}-0.3 \\
-1.3\end{array}$ \\
\hline $8 I$ & $\begin{array}{l}\text { A } \\
\text { B }\end{array}$ & $\begin{array}{r}0.055 \\
-0.209\end{array}$ & $\begin{array}{c}2.2 \\
-7.7^{\star}\end{array}$ & $\begin{array}{r}0.999 \\
-0.998\end{array}$ & $\begin{array}{l}0.2 \\
0.4\end{array}$ \\
\hline $8 P$ & $\begin{array}{l}A \\
B\end{array}$ & $\begin{array}{r}-0.125 \\
0.067\end{array}$ & $\begin{array}{r}-13.9 * \\
11.2^{\star}\end{array}$ & $\begin{array}{l}0.999 \\
0.999\end{array}$ & $\begin{array}{l}0.5 \\
1.0\end{array}$ \\
\hline $9 \mathrm{~V}$ & $\begin{array}{l}\mathrm{A} \\
\mathrm{B}\end{array}$ & $\begin{array}{r}-0.008 \\
0.025\end{array}$ & $\begin{array}{r}-0.6 \\
2.5\end{array}$ & $\begin{array}{l}0.999 \\
1.003\end{array}$ & $\begin{array}{r}0.5 \\
-1.5\end{array}$ \\
\hline $9 W$ & $\begin{array}{l}\text { A } \\
\text { B }\end{array}$ & $\begin{array}{r}-0.023 \\
0.121\end{array}$ & $\frac{-1.9}{15.1}$ & $\begin{array}{l}0.998 \\
0.998\end{array}$ & $\begin{array}{l}1.0 \\
1.0\end{array}$ \\
\hline $10 I$ & $\begin{array}{l}\mathrm{A} \\
\mathrm{B}\end{array}$ & $\begin{array}{r}0.079 \\
-0.160\end{array}$ & $\begin{array}{r}4.2^{\star} \\
-17.8^{\star}\end{array}$ & $\begin{array}{l}1.000 \\
1.008\end{array}$ & $\begin{array}{c}0.0 \\
-4.0 \text { * }\end{array}$ \\
\hline $11 \mathrm{~F}$ & $\begin{array}{l}\mathrm{A} \\
\mathrm{B}\end{array}$ & $\begin{array}{r}-0.172 \\
0.215\end{array}$ & $\begin{array}{r}-4.9 \star \\
7.7 \star\end{array}$ & $\begin{array}{l}1.000 \\
1.012\end{array}$ & $\begin{array}{l}0.0 \\
-2.4^{\star}\end{array}$ \\
\hline $11 I$ & $\begin{array}{l}\mathrm{A} \\
\mathrm{B}\end{array}$ & $\begin{array}{r}0.245 \\
-0.135\end{array}$ & $\begin{array}{l}18.8^{\star} \\
-7.9 *\end{array}$ & $\begin{array}{l}0.999 \\
0.995\end{array}$ & $\begin{array}{l}0.5 \\
1.7\end{array}$ \\
\hline $13 \mathrm{C}$ & $\begin{array}{l}\mathrm{A} \\
\mathrm{B}\end{array}$ & $\begin{array}{l}0.193 \\
0.009\end{array}$ & $\begin{array}{c}19.3^{\star} \\
0.5\end{array}$ & $\begin{array}{l}0.995 \\
1.000\end{array}$ & $\begin{array}{l}2.5^{\star} \\
0.0\end{array}$ \\
\hline $13 I$ & $\begin{array}{l}\mathrm{A} \\
\mathrm{B}\end{array}$ & $\begin{array}{r}0.157 \\
-0.209\end{array}$ & $\begin{array}{r}13.1 \star \\
-10.0 \star\end{array}$ & $\begin{array}{l}1.010 \\
1.010\end{array}$ & $\begin{array}{l}-5.0 \star \\
-2.5 \star\end{array}$ \\
\hline $14 \mathrm{C}$ & $\begin{array}{l}\mathrm{A} \\
\mathrm{B}\end{array}$ & $\begin{array}{l}0.073 \\
0.307\end{array}$ & $\begin{array}{r}4.3 * \\
15.3\end{array}$ & $\begin{array}{l}0.995 \\
0.996\end{array}$ & $\begin{array}{l}1.7 \\
1.0\end{array}$ \\
\hline $15 B$ & $\begin{array}{l}\mathrm{A} \\
\mathrm{B}\end{array}$ & $\begin{array}{r}0.037 \\
-0.001\end{array}$ & $\begin{array}{r}2.2 \\
-0.1\end{array}$ & $\begin{array}{l}0.992 \\
0.981\end{array}$ & $\begin{array}{l}2.7 \text { * } \\
6.3 \text { * }\end{array}$ \\
\hline
\end{tabular}

* Exceeds critical value for two-sided test at the 0.95 probability level. 
Table 6 - Frequency of Differences Between Participant's Linewidth Measurements and NBS Values on Opaque Lines (Row C) ${ }^{\dagger}$

Number of Differences

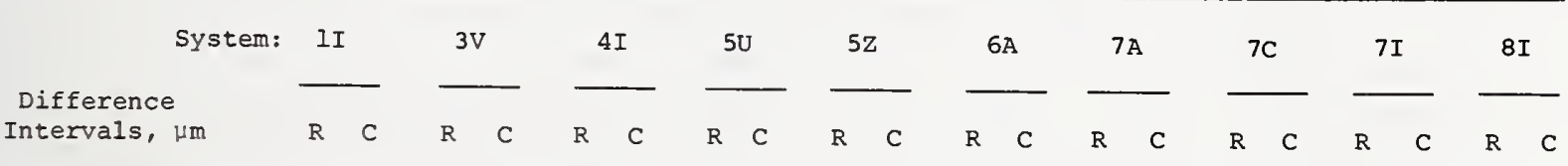

\begin{tabular}{|c|c|c|}
\hline-1.00 & to & -0.95 \\
\hline-0.95 & to & -0.90 \\
\hline-0.90 & to & -0.85 \\
\hline-0.85 & to & -0.80 \\
\hline-0.80 & to & -0.75 \\
\hline-0.75 & to & -0.70 \\
\hline-0.70 & to & -0.65 \\
\hline-0.65 & to & -0.60 \\
\hline-0.60 & to & -0.55 \\
\hline-0.55 & to & -0.50 \\
\hline-0.50 & to & -0.45 \\
\hline-0.45 & to & -0.40 \\
\hline-0.40 & to & -0.35 \\
\hline-0.35 & to & -0.30 \\
\hline-0.30 & to & -0.25 \\
\hline-0.25 & to & -0.20 \\
\hline-0.20 & to & -0.15 \\
\hline-0.15 & to & -0.10 \\
\hline-0.10 & to & -0.05 \\
\hline-0.05 & to & 0.00 \\
\hline 0.00 & to & 0.05 \\
\hline 0.05 & to & 0.10 \\
\hline 0.10 & to & 0.15 \\
\hline 0.15 & to & 0.20 \\
\hline 0.20 & to & 0.25 \\
\hline 0.25 & to & 0.30 \\
\hline 0.30 & to & 0.35 \\
\hline 0.35 & to & 0.40 \\
\hline 0.40 & to & 0.45 \\
\hline 0.45 & to & 0.50 \\
\hline 0.50 & to & 0.55 \\
\hline 0.55 & to & 0.60 \\
\hline 0.60 & to & 0.65 \\
\hline 0.65 & to & 0.70 \\
\hline 0.70 & to & 0.75 \\
\hline 0.75 & to & 0.80 \\
\hline 0.80 & to & 0.85 \\
\hline 0.85 & to & 0.90 \\
\hline 0.90 & & 0.95 \\
\hline 0.95 & & 1.00 \\
\hline
\end{tabular}

\begin{tabular}{|c|c|c|c|c|c|c|c|}
\hline ४* & \# & $\#$ * & \#* & $\#$ & \# \% & 2 & ** \\
\hline ** & ** & $* *$ & * & ** & ** & $\star *$ & ‡ * \\
\hline \# & * \# & $\#$ * & \#* & \#* & * * & 6 & ** \\
\hline$* *$ & ** & $* *$ & $* *$ & $* *$ & $\# *$ & 1 & $\#$ \\
\hline ** & ** & $* *$ & ** & ** & \# & 1 & $* *$ \\
\hline ** & $* *$ & $* *$ & * & *4 & $\star *$ & 5 & * * \\
\hline ** & * * & ** & \# * & $\star \star *$ & $\star \star$ & 3 & * \\
\hline$* *$ & $* *$ & $* *$ & $* *$ & ** & $* \star$ & 4 & $\#$ \\
\hline \# * & ** & $\star *$ & $* *$ & $* *$ & $\star \star \star$ & 3 & ** \\
\hline ** & * * & $* *$ & $\#$ & * * & $*$ & 1 & $\star *$ \\
\hline ** & ** & ** & $* *$ & * & * * & 2 & * * \\
\hline ** & ** & * * & \# * & * & $\star *$ & 1 & \# \\
\hline ** & $\# \star$ & \# & $4 *$ & ** & 1 & 6 & ** \\
\hline$\star \star$ & \# * & ** & \#* & ** & \#* & 7 & * * \\
\hline ** & ‡ & * & \#4 & $*$ & 1 & 5 & 屯 \\
\hline ** & * * & ** & ** & $* \star$ & 5 & 6 & 1 \\
\hline \# * & 2 & 屯 & 4 & 1 & 7 & 2 & 4 \\
\hline 屯* & 8 & * * & $\star \star$ & $* *$ & 10 & 10 & 5 \\
\hline * & 11 & ** & ‡屯 & 2 & 8 & 1 & 14 \\
\hline 1 & 13 & 30 & 26 & 5 & 14 & 屯 & 22 \\
\hline 2 & 8 & 50 & 54 & 9 & 12 & ‡ * & 10 \\
\hline 11 & 13 & $* *$ & $* *$ & 12 & 10 & * * & 15 \\
\hline 13 & 5 & * * & $\star *$ & 7 & 2 & ** & 6 \\
\hline 12 & * & * * & $* *$ & 15 & 5 & $* *$ & 2 \\
\hline 8 & $\#$ \# & $\# \star$ & $\star *$ & 12 & * * & $*$ * & 1 \\
\hline 13 & $* *$ & ** & \# * & 6 & $*$ & * * & * \\
\hline$* 4$ & * * & $* *$ & $* *$ & 4 & 3 & $* *$ & $* \neq$ \\
\hline * * & ** & $\star *$ & $\star \star$ & 3 & $* \star$ & $* *$ & $* *$ \\
\hline ** & ** & \# & \# * & * * & 1 & $\neq \#$ & \# \\
\hline ** & $* *$ & * * & $* *$ & 1 & $* *$ & † * & 屯 \\
\hline$* \star *$ & 屯* & $* *$ & $* *$ & 2 & 1 & * * & \#4 \\
\hline * * & * * & * * & $* *$ & 1 & $\star *$ & 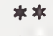 & \# \\
\hline ** & $* *$ & * * & $* *$ & $\star *$ & $*$ & $\star *$ & $\neq$ \\
\hline ** & ** & \% & ** & 1 & $* *$ & 屯* & $*$ \\
\hline \# & ** & ** & 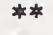 & ** & $\star *$ & \# & * \\
\hline * * & ** & ** & $\star *$ & ** & $\star \star$ & * * & ** \\
\hline ** & \# * & * * & ** & * & $* *$ & \#* & 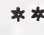 \\
\hline ** & $* *$ & ** & * & $* 4$ & * * & ** & ‡ \\
\hline ** & * & ** & ** & $\# *$ & ** & $\star *$ & * \\
\hline ** & \#\# & $* *$ & $\star \star$ & ** & 屯 & $* *$ & $\star *$ \\
\hline
\end{tabular}

\begin{tabular}{|c|c|c|c|c|c|c|c|}
\hline ** & 1 & ** & $* *$ & * * & * * & * * & ** \\
\hline 4 & 4* & ** & \# & ** & * & * & \#屯 \\
\hline \#* & * & * * & * * & ** & * * & * * & 末 * \\
\hline 1 & 1 & $\star \star$ & $\star \star$ & ** & $* *$ & * * & ** \\
\hline * * & $\star *$ & * * & $* *$ & $* *$ & ** & ** & ** \\
\hline ** & * * & $* *$ & * * & 屯 & $* *$ & $\star *$ & $* 4$ \\
\hline$\star \star *$ & ** & $\star *$ & $* *$ & * * & ** & 廿* & \#女 \\
\hline ** & * * & ** & ** & ** & * * & * * & $\star \star$ \\
\hline ** & ** & ** & ** & ઐ* & ** & ** & ** \\
\hline$* *$ & $\star \star$ & * * & ** & $* *$ & ‡ & $\# \star$ & $\star \star$ \\
\hline * * & \#* & * * & * & ** & $\star \star \star$ & $\star \star$ & $\star \star$ \\
\hline ** & ** & $\# \star$ & 屯 & ㅎ & $* *$ & ** & 屯* \\
\hline$* *$ & 屯* & $* *$ & \#* & * & $* *$ & $\star *$ & ** \\
\hline 3 & 2 & 1 & ** & \# & $\star \star$ & * & \#* \\
\hline * & 1 & * * & 1 & ** & $* *$ & $\star \star$ & $* 4$ \\
\hline 1 & 1 & $\star *$ & $\star \star$ & ** & ऋ * & 1 & 1 \\
\hline 2 & 3 & 2 & 2 & * & 1 & 3 & 2 \\
\hline 6 & 3 & 3 & 8 & \# & 4 & 12 & 9 \\
\hline 11 & 11 & 4 & 9 & 6 & 9 & 16 & 15 \\
\hline 27 & 26 & 8 & 15 & 6 & 13 & 9 & 12 \\
\hline 9 & 14 & 12 & 21 & 10 & 22 & 10 & 10 \\
\hline 15 & 11 & 10 & 11 & 21 & 19 & 7 & 6 \\
\hline ** & 2 & 13 & 7 & 15 & 3 & 7 & 9 \\
\hline ** & $* *$ & 11 & 3 & 13 & 2 & 4 & 5 \\
\hline$* *$ & $\#$ & 7 & 1 & 3 & 6 & * * & \#4 \\
\hline ** & 屯 & 3 & 2 & 3 & 1 & 屯 * & $* \star$ \\
\hline ‡* & * * & 4 & $* \star$ & 3 & 屯 & 屯 * & *4 \\
\hline \% * & 屯 & * & * * & ** & $\star \star$ & * * & \# \\
\hline * * & \# * & 1 & ** & \#屯 & 屯 & 屯 & ** \\
\hline * * & * * & 1 & $\star *$ & $\star \star \star$ & $\star *$ & $\star \star \star$ & $* \star$ \\
\hline * * & * * & ** & * * & $*$ & 屯 屯 & * * & $\# *$ \\
\hline 1 & 1 & $\star *$ & \# * & $\star *$ & 屯 & 屯 & ‡ \\
\hline \#* & * * & 屯 & * * & 屯 * & \# & \# & 屯* \\
\hline$\# *$ & $\# *$ & 屯 & ** & ** & * * & $\star \star$ & \# \\
\hline$* 4$ & $*$ * & * * & ** & \#\# & 屯 & 屯 屯 & $\star *$ \\
\hline * & * & * & $*$ * & \# & $\star \star$ & * * & 末 \\
\hline$* *$ & * * & $\star \star$ & $\star \star \star$ & ‡ & 末 * & 屯 & $\star \star \star$ \\
\hline ‡* & $\star \star$ & 屯 & \#* & \#* & $* *$ & * * & \#‡ \\
\hline ** & ** & * * & $\star \star \star$ & ** & \# & * * & * \\
\hline$\not *$ & 4 & 屯 & $* *$ & * * & $\star *$ & 屯 * & \#* \\
\hline
\end{tabular}

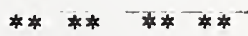

*

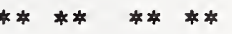

* *4 ** **

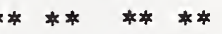

女* *

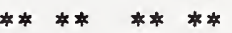

** **

** *末

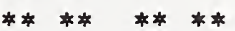

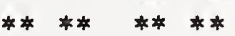

\#*\#華

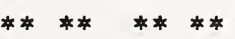

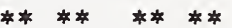

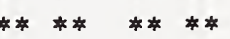

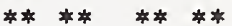

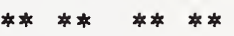

*** ** 6

* 55516

* * $16 \quad \frac{17}{22} \quad 17$

* 1699

* 792

1122 \%

181 * * 1

$23 *$ 1 * *

121 ** *

4 ** *\# * *

** ** ** **

$1 * *$ ** **

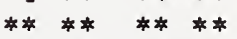

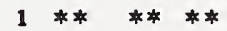

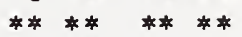

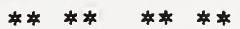

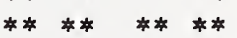

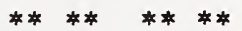

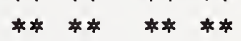

********

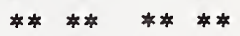

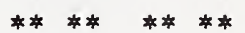

Number of Differences

Within $\pm 1.0 \mu \mathrm{m}$

$\begin{array}{llllllllllllllllllll}60 & 60 & 90 & 80 & 80 & 80 & 66 & 80 & 76 & 77 & 80 & 80 & 80 & 80 & 69 & 69 & 70 & 70 & 60 & 60\end{array}$

Number of Differences

Greater than $\pm 1.0 \mu \mathrm{m}$

t The left column $(R)$ for each system contains the differences for the raw data, and the right column (C) contains the differences for the corrected data. A double asterisk denotes the absence of a difference value. 


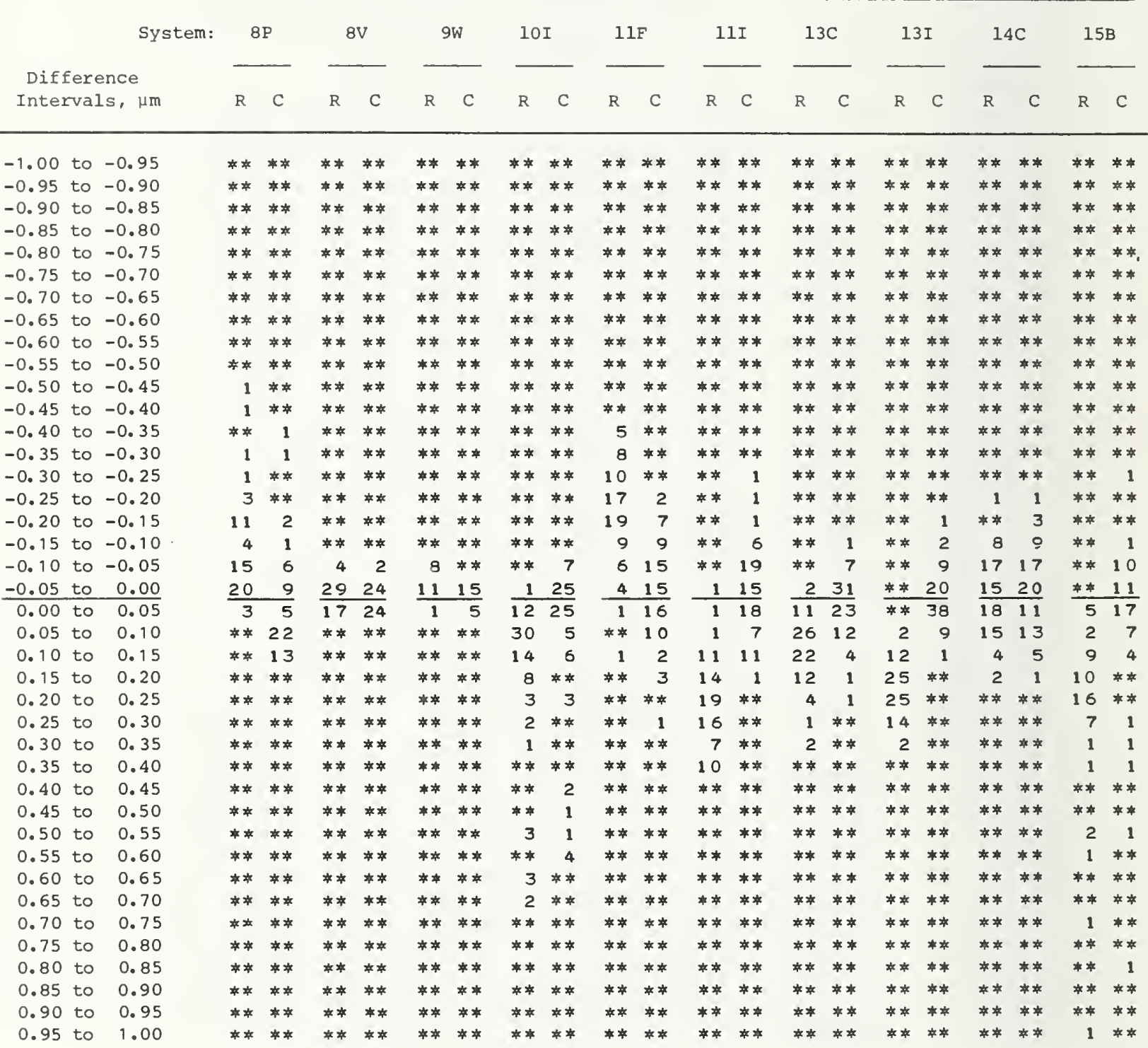

Number of Differences

Within $\pm 1.0 \mu \mathrm{m}$ $\begin{array}{llllll}60 & 60 & 50 & 50 & 20 & 20\end{array}$

Number of Differences Greater than $\pm 1.0 \mu \mathrm{m}$

$7979 \quad 80$

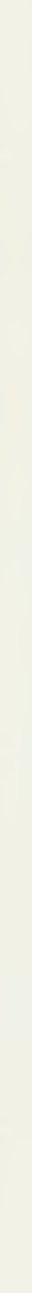


Table 7 - Frequency of Differences Between Participant's Linewidth Measurements and NBS Values on Clear Lines (Row D) ${ }^{+}$

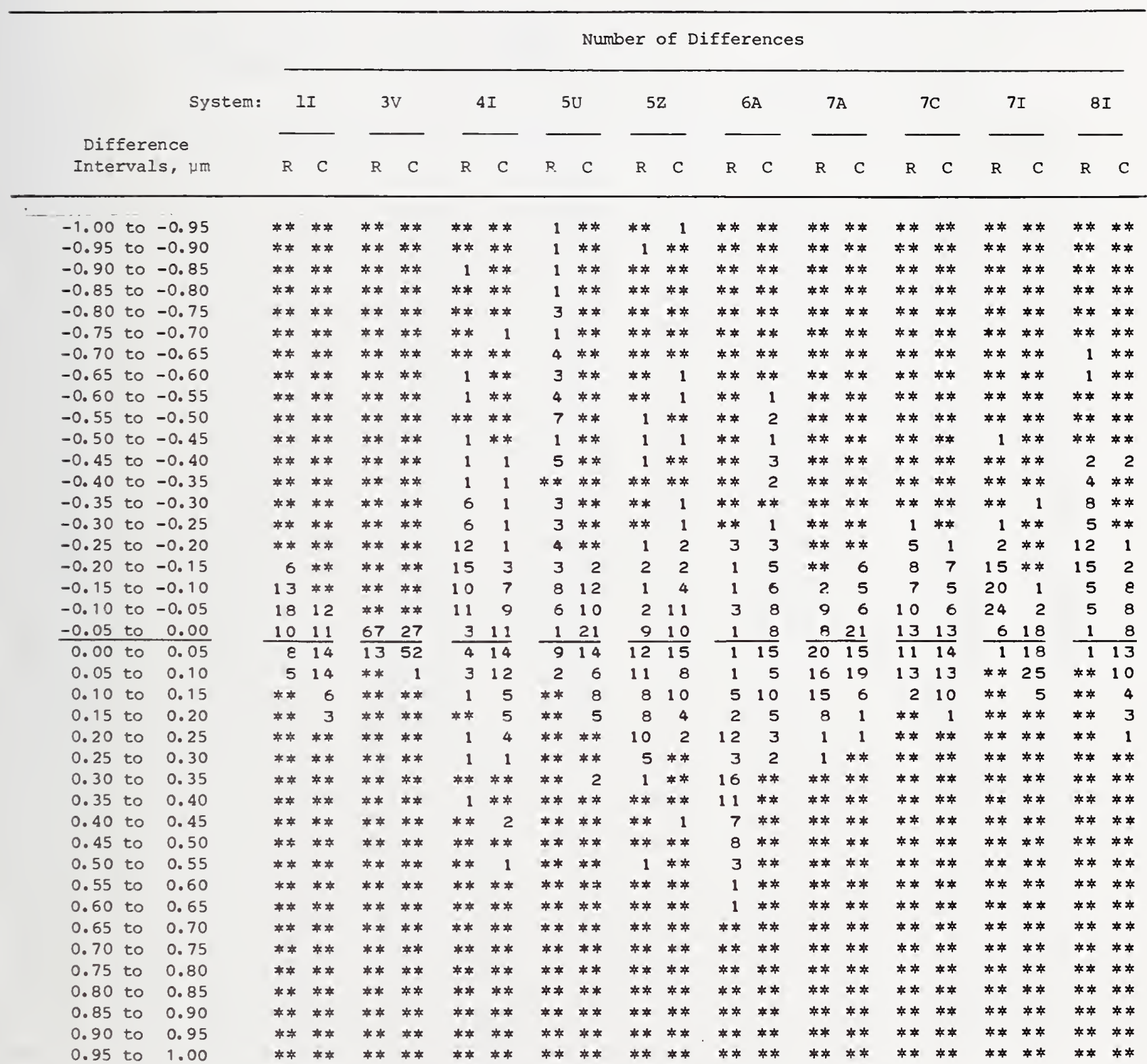

Number of Differences

Within $\pm 1.0 \mu \mathrm{m}$

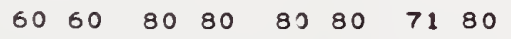

$7575 \quad 8080$

$80 \quad 80 \quad 70 \quad 70$

7070

6060

Number of Differences

Greater than $\pm 1.0 \mu \mathrm{m}$

\author{
00
}

\begin{abstract}
00
\end{abstract}

\begin{abstract}
0090
\end{abstract}
55

00

00

$\begin{array}{llllll}0 & 0 & 0 & 0 & 0 & 0\end{array}$

T The left column (R) for each system contains the differences for the raw data, and the right column (C) contains the differences for the corrected data. A double asterisk denotes the absence of a difference value. 
Table 7 - Concluded

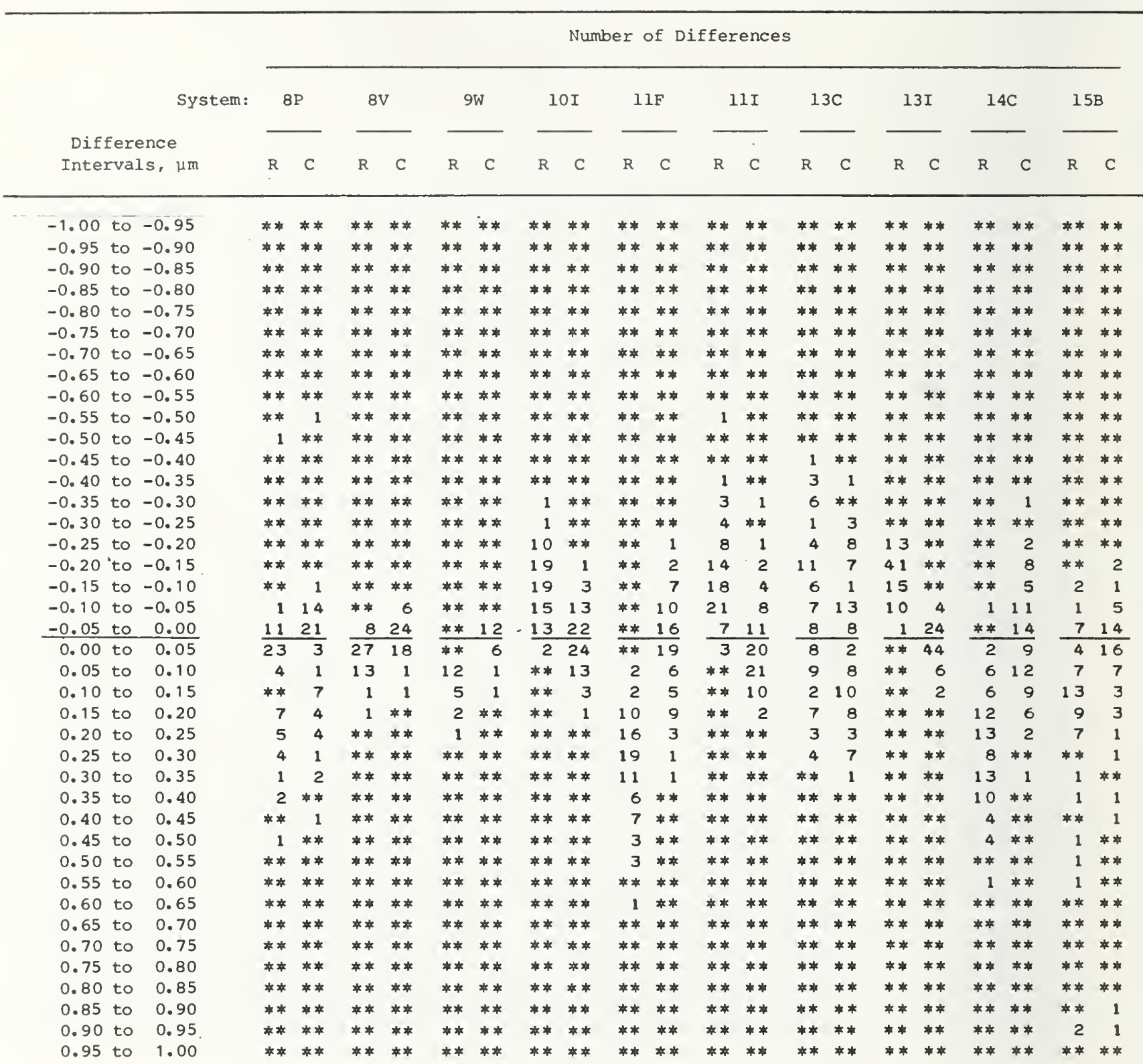

Number of Differences

Within $\pm 1.0 \mu \mathrm{m}$

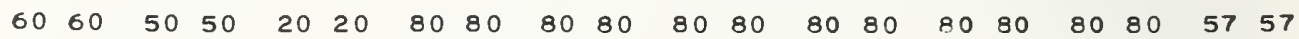

Number of Differences

Greater than $\pm 1.0 \mu \mathrm{I}$.

$\begin{array}{lllllll}0 & 0 & 0 & 0 & 0 & 0 & 0\end{array}$

$0 \quad 0$

0

00

$0 \quad 0$

$0 \quad 0$

33 
Table 8 - Random Error Limits for Linewidth Measurements Corrected by Linear Calibration Curve, Process Standard Deviations, and Degrees of Freedom

\begin{tabular}{|c|c|c|c|c|c|c|}
\hline \multirow{2}{*}{$\begin{array}{l}\text { Measurement } \\
\text { System }\end{array}$} & \multicolumn{3}{|c|}{$\begin{array}{c}\text { Row } A \\
\text { (opaque lines) }\end{array}$} & \multicolumn{3}{|c|}{$\begin{array}{c}\text { Row B } \\
\text { (clear lines) }\end{array}$} \\
\hline & $E(\mu m)$ & $s_{L}(\mu \mathrm{m})$ & $v$ & $E(\mu \mathrm{m})$ & $\mathrm{s}_{L}(\mu \mathrm{m})$ & $v$ \\
\hline $1 I$ & 0.28 & 0.082 & 58 & 0.21 & 0.059 & 58 \\
\hline $3 \mathrm{~V}$ & 0.04 & 0.013 & 77 & 0.21 & 0.014 & 78 \\
\hline $4 I$ & 0.35 & 0.105 & 77 & 0.41 & 0.122 & 77 \\
\hline $5 U$ & 0.32 & 0.079 & 77 & 0.47 & 0.119 & 77 \\
\hline $5 z$ & 0.37 & 0.108 & 68 & 0.33 & 0.099 & 60 \\
\hline $6 \mathrm{~A}$ & 0.37 & 0.105 & 78 & 0.35 & 0.105 & 68 \\
\hline $7 \mathrm{~A}$ & 0.15 & 0.045 & 68 & 0.27 & 0.082 & 77 \\
\hline $7 C$ & 0.32 & 0.094 & 68 & 0.37 & 0.111 & 68 \\
\hline $7 I$ & 0.18 & 0.054 & 67 & 0.15 & 0.046 & 68 \\
\hline $8 I$ & 0.31 & 0.090 & 58 & 0.31 & 0.089 & 58 \\
\hline $8 P$ & 0.21 & 0.060 & 55 & 0.22 & 0.066 & 58 \\
\hline $9 \mathrm{~V}$ & 0.11 & 0.030 & 48 & 0.13 & 0.037 & 48 \\
\hline $9 W$ & 0.11 & 0.026 & 18 & 0.17 & 0.039 & 18 \\
\hline $10 I$ & 0.14 & 0.041 & 78 & 0.17 & 0.051 & 77 \\
\hline $11 \mathrm{~F}$ & 0.32 & 0.096 & 78 & 0.32 & 0.099 & 78 \\
\hline $11 I$ & 0.25 & 0.076 & 78 & 0.25 & 0.074 & 78 \\
\hline $13 \mathrm{C}$ & 0.13 & 0.040 & 68 & 0.56 & 0.169 & 78 \\
\hline $13 I$ & 0.14 & 0.043 & 77 & 0.16 & 0.048 & 78 \\
\hline $14 \mathrm{C}$ & 0.28 & 0.084 & 78 & 0.37 & 0.111 & 78 \\
\hline $15 B$ & 0.24 & 0.066 & 48 & 0.34 & 0.094 & 48 \\
\hline
\end{tabular}


Table 9 - F Statistics for Detecting Between-Day Differences in Linewidth Calibration Curves, Degrees of Freedom, and Pooled Standard Deviations ${ }^{\dagger}$

\begin{tabular}{|c|c|c|c|c|c|c|c|c|c|}
\hline \multirow{2}{*}{$\begin{array}{l}\text { Mea surement } \\
\text { System }\end{array}$} & \multirow{2}{*}{ Row } & \multicolumn{4}{|c|}{ Operator A } & \multicolumn{4}{|c|}{ Operator B } \\
\hline & & $F$ & $v_{1}$ & $v_{2}$ & ${ }^{\mathrm{S}} \mathrm{P}$ & $\bar{F}$ & $v_{1}$ & $v_{2}$ & ${ }^{s_{P}}$ \\
\hline \multirow[t]{4}{*}{$1 I$} & $\mathrm{~A}$ & 2.1 & 4 & 24 & 0.054 & $5.2^{\star}$ & 4 & 24 & 0.044 \\
\hline & B & 1.5 & 4 & 24 & 0.033 & 1.5 & 4 & 24 & 0.036 \\
\hline & C & 0.8 & 4 & 24 & 0.059 & $16.4^{\star}$ & 4 & 24 & 0.037 \\
\hline & $\mathrm{D}$ & 2.7 & 4 & 24 & 0.040 & 3.2 & 4 & 24 & 0.026 \\
\hline \multirow[t]{4}{*}{$3 \mathrm{~V}$} & $A$ & 0.6 & 6 & 32 & 0.011 & 1.8 & 6 & 32 & 0.015 \\
\hline & $\mathrm{B}$ & 0.3 & 6 & 32 & 0.013 & 2.9 & 6 & 32 & 0.012 \\
\hline & $C$ & 0.2 & 6 & 32 & 0.012 & 1.5 & 6 & 32 & 0.018 \\
\hline & D & 0.4 & 6 & 32 & 0.012 & 3.2 & 6 & 32 & 0.015 \\
\hline \multirow[t]{4}{*}{$4 I$} & A & 0.6 & 6 & 32 & 0.143 & $6.3 *$ & 6 & 32 & 0.068 \\
\hline & B & 0.3 & 6 & 32 & 0.172 & $5.8 *$ & 6 & 32 & 0.075 \\
\hline & $\mathrm{C}$ & $9.8 *$ & 6 & 32 & 0.116 & 2.8 & 6 & 32 & 0.101 \\
\hline & $\mathrm{D}$ & $5.3 \star$ & 6 & 32 & 0.156 & 1.7 & 6 & 32 & 0.094 \\
\hline \multirow[t]{4}{*}{$5 \mathrm{U}$} & $A$ & 2.7 & 6 & 32 & 0.070 & 3.1 & 6 & 32 & 0.070 \\
\hline & B & 1.6 & 6 & 32 & 0.078 & $12.2^{\star}$ & 6 & 31 & 0.081 \\
\hline & $\mathrm{C}$ & 0.8 & 6 & 32 & 0.079 & 1.7 & 6 & 32 & 0.071 \\
\hline & $D$ & 0.9 & 6 & 32 & 0.075 & $10.4^{\star}$ & 6 & 32 & 0.061 \\
\hline \multirow[t]{4}{*}{$5 z$} & A & 1.4 & 6 & 32 & 0.115 & 47. $0^{\star}$ & 6 & 32 & 0.159 \\
\hline & B & $5.4^{\star}$ & 6 & 32 & 0.082 & $336.9 *$ & 6 & 32 & 0.068 \\
\hline & $C$ & 4.1 & 6 & 32 & 0.059 & $11.9^{\star}$ & 6 & 32 & 0.280 \\
\hline & $D$ & 2.8 & 6 & 32 & 0.103 & 110.6 * & 6 & 32 & 0.126 \\
\hline \multirow[t]{4}{*}{$6 \mathrm{~A}$} & $A$ & $4.8^{\star}$ & 6 & 32 & 0.081 & 2.2 & 6 & 32 & 0.084 \\
\hline & B & 2.7 & 6 & 32 & 0.079 & $21.5^{\star}$ & 6 & 32 & 0.096 \\
\hline & $C$ & $4.6^{\star}$ & 6 & 32 & 0.065 & 1.4 & 6 & 32 & 0.087 \\
\hline & D & 3.2 & 6 & 32 & 0.112 & 39.5 * & 6 & 32 & 0.077 \\
\hline \multirow[t]{4}{*}{$7 A$} & $A$ & 43.4 * & 6 & 32 & 0.039 & 2.9 & 6 & 32 & 0.038 \\
\hline & B & $9.3 *$ & 6 & 32 & 0.057 & $18.6^{*}$ & 6 & 32 & 0.039 \\
\hline & $C$ & $32.2^{\star}$ & 6 & 32 & 0.038 & $3.7 \star$ & 6 & 32 & 0.054 \\
\hline & D & $13.2^{\star}$ & 6 & 32 & 0.055 & $16 \cdot 3^{\star}$ & 6 & 32 & 0.040 \\
\hline \multirow[t]{4}{*}{$7 \mathrm{C}$} & A & $19.4 *$ & 4 & 24 & 0.053 & 2.4 & 6 & 32 & 0.076 \\
\hline & B & $9.7 \star$ & 4 & 24 & 0.065 & $5.8 *$ & 6 & 32 & 0.062 \\
\hline & C & $12.1 *$ & 4 & 23 & 0.065 & $4.4^{\star}$ & 6 & 32 & 0.069 \\
\hline & $D$ & $8.9^{\star}$ & 4 & 24 & 0.054 & $6.3 *$ & 6 & 32 & 0.056 \\
\hline \multirow[t]{4}{*}{$7 I$} & $\mathrm{~A}$ & 3.1 & 4 & 24 & 0.039 & 2.0 & 6 & 32 & 0.041 \\
\hline & B & 1.9 & 4 & 24 & 0.043 & 1.5 & 6 & 32 & 0.045 \\
\hline & $\mathrm{C}$ & $9.3 *$ & 4 & 24 & 0.045 & $5.2^{\star}$ & 6 & 32 & 0.036 \\
\hline & $D$ & 0.6 & 4 & 24 & 0.050 & $4.6^{\star}$ & 6 & 32 & 0.060 \\
\hline
\end{tabular}

IInsufficient data to determine statistics for measurement systems $9 \mathrm{~V}$ and $9 \mathrm{~W}$.

* Significant at the 0.99 probability level. 
Table 9 - Concluded

\begin{tabular}{|c|c|c|c|c|c|c|c|c|c|}
\hline \multirow{2}{*}{$\begin{array}{l}\text { Measurement } \\
\text { System }\end{array}$} & \multirow{2}{*}{ Row } & \multicolumn{4}{|c|}{ Operator $\mathrm{A}$} & \multicolumn{4}{|c|}{ Operator B } \\
\hline & & $\mathrm{F}$ & $v_{1}$ & $v_{2}$ & $\mathrm{~s}_{\mathrm{P}}$ & $\mathrm{F}$ & $v_{1}$ & $v_{2}$ & $\mathrm{~s}_{\mathrm{P}}$ \\
\hline \multirow[t]{4}{*}{$8 I$} & A & $8.8^{*}$ & 4 & 24 & 0.068 & 0.2 & 4 & 24 & 0.059 \\
\hline & B & 1.6 & 4 & 24 & 0.086 & 0.9 & 4 & 24 & 0.052 \\
\hline & $\mathrm{C}$ & $5 \cdot 9 *$ & 4 & 24 & 0.056 & 1.2 & 4 & 24 & 0.048 \\
\hline & $\mathrm{D}$ & $4.2^{\star}$ & 4 & 24 & 0.107 & 1.4 & 4 & 24 & 0.050 \\
\hline \multirow[t]{4}{*}{$8 \mathrm{P}$} & A & $7.2^{\star}$ & 4 & 24 & 0.076 & 1.1 & 4 & 24 & 0.083 \\
\hline & B & $21.9 *$ & 4 & 24 & 0.030 & 0.4 & 4 & 24 & 0.026 \\
\hline & C & $6.5^{\star}$ & 4 & 24 & 0.081 & 2.9 & 4 & 24 & 0.026 \\
\hline & $\mathrm{D}$ & $16.0 *$ & 4 & 24 & 0.074 & 0.3 & 4 & 24 & 0.101 \\
\hline \multirow[t]{4}{*}{$10 I$} & A & 1.7 & 6 & 32 & 0.034 & 1.9 & 6 & 32 & 0.044 \\
\hline & $\mathrm{B}$ & $4.9 *$ & 6 & 32 & 0.038 & 4.0 * & 6 & 32 & 0.048 \\
\hline & $\mathrm{C}$ & 0.6 & 6 & 32 & 0.163 & 0.0 & 6 & 31 & 0.192 \\
\hline & $\mathrm{D}$ & $3.5 *$ & 6 & 32 & 0.049 & 4.7 * & 6 & 32 & 0.050 \\
\hline \multirow[t]{4}{*}{$11 \mathrm{~F}$} & $A$ & 2.2 & 6 & 32 & 0.092 & 1.1 & 6 & 32 & 0.092 \\
\hline & B & 0.3 & 6 & 32 & 0.115 & 2.8 & 6 & 32 & 0.049 \\
\hline & $\mathrm{C}$ & 1.1 & 6 & 32 & 0.115 & 2.4 & 6 & 32 & 0.057 \\
\hline & D & 1.4 & 6 & 32 & 0.110 & 1.8 & 6 & 32 & 0.064 \\
\hline \multirow[t]{4}{*}{$11 I$} & $A$ & 0.4 & 6 & 32 & 0.081 & $6.5^{\star}$ & 6 & 32 & 0.055 \\
\hline & B & 2.1 & 6 & 32 & 0.068 & 5.2 * & 6 & 32 & 0.054 \\
\hline & $\mathrm{C}$ & 1.1 & 6 & 32 & 0.097 & 2.1 & 6 & 32 & 0.064 \\
\hline & $\mathrm{D}$ & 3.0 & 6 & 32 & 0.073 & 2.6 & 6 & 32 & 0.082 \\
\hline \multirow[t]{4}{*}{$13 C$} & $\mathrm{~A}$ & $41 \cdot 6 *$ & 6 & 32 & 0.029 & 2.9 & 6 & 32 & 0.038 \\
\hline & B & 33.7 * & 6 & 32 & 0.081 & $44.5 *$ & 6 & 32 & 0.037 \\
\hline & $\mathrm{C}$ & $18.7 \star$ & 6 & 32 & 0.035 & 2.0 & 6 & 32 & 0.039 \\
\hline & $\mathrm{D}$ & $69.6 *$ & 6 & 32 & 0.057 & $36.5 *$ & 6 & 32 & 0.039 \\
\hline \multirow[t]{4}{*}{$13 I$} & A & $8.8^{\star}$ & 6 & 32 & 0.031 & 2.3 & 6 & 32 & 0.025 \\
\hline & B & 2.5 & 6 & 32 & 0.050 & 0.8 & 6 & 32 & 0.042 \\
\hline & $\mathrm{C}$ & $11 \cdot 3^{\star}$ & 6 & 32 & 0.034 & $5 \cdot 5^{\star}$ & 6 & 32 & 0.029 \\
\hline & D & 2.6 & 6 & 32 & 0.040 & 1.9 & 6 & 32 & 0.028 \\
\hline \multirow[t]{4}{*}{$14 \mathrm{C}$} & $\mathrm{A}$ & 2.8 & 6 & 32 & 0.083 & 10.9 * & 6 & 32 & 0.043 \\
\hline & B & 2.4 & 6 & 32 & 0.110 & 9.6 * & 6 & 32 & 0.055 \\
\hline & $\mathrm{C}$ & $6.9 *$ & 6 & 32 & 0.066 & $4.7 \star$ & 6 & 32 & 0.050 \\
\hline & D & $6.0 *$ & 6 & 32 & 0.098 & $11.8^{*}$ & 6 & 32 & 0.062 \\
\hline \multirow[t]{4}{*}{$15 B$} & A & 3.5 & 6 & 32 & 0.057 & $656.7 *$ & 6 & 32 & 0.046 \\
\hline & B & $16.2^{*}$ & 6 & 32 & 0.051 & 203.3 * & 6 & 32 & 0.081 \\
\hline & $\mathrm{C}$ & 2.0 & 6 & 32 & 0.065 & $162.7 *$ & 6 & 32 & 0.093 \\
\hline & D & 2.2 & 6 & 32 & 0.061 & 282.8 * & 6 & 32 & 0.064 \\
\hline
\end{tabular}

* Significant at the 0.99 probability level. 
Table 10 - Standard Deviations of Repetition, Process Standard Deviations, Degrees of Freedom, and F Statistics for Testing Linearity of Linewidth Calibration Model ${ }^{\dagger}$

\begin{tabular}{|c|c|c|c|c|c|c|}
\hline \multirow{2}{*}{$\begin{array}{l}\text { Measurement } \\
\text { System }\end{array}$} & \multirow{2}{*}{ Row } & \multicolumn{2}{|c|}{ Repetitions } & \multicolumn{2}{|c|}{ Linear Fit } & \multirow{2}{*}{$F$} \\
\hline & & $s_{R}(\mu \mathrm{m})$ & $v$ & $s_{L}(\mu m)$ & $v$ & \\
\hline $1 \mathrm{I}$ & $\begin{array}{l}\text { A } \\
\text { B }\end{array}$ & $\begin{array}{l}0.0803 \\
0.0565\end{array}$ & $\begin{array}{l}50 \\
50\end{array}$ & $\begin{array}{l}0.0815 \\
0.0589\end{array}$ & $\begin{array}{l}58 \\
58\end{array}$ & $\begin{array}{l}1.2 \\
1.6\end{array}$ \\
\hline $3 V$ & $\begin{array}{l}\text { A } \\
\text { B }\end{array}$ & $\begin{array}{l}0.0083 \\
0.0097\end{array}$ & $\begin{array}{l}69 \\
70\end{array}$ & $\begin{array}{l}0.0126 \\
0.0137\end{array}$ & $\begin{array}{l}77 \\
78\end{array}$ & $\begin{array}{l}13.8 * \\
10.5 *\end{array}$ \\
\hline $4 I$ & $\begin{array}{l}\text { A } \\
\text { B }\end{array}$ & $\begin{array}{l}0.1009 \\
0.1211\end{array}$ & $\begin{array}{l}69 \\
69\end{array}$ & $\begin{array}{l}0.1051 \\
0.1225\end{array}$ & $\begin{array}{l}77 \\
77\end{array}$ & $\begin{array}{l}1.8 \\
1.2\end{array}$ \\
\hline $5 U$ & $\begin{array}{l}\text { A } \\
\text { B }\end{array}$ & $\begin{array}{l}0.0861 \\
0.1322\end{array}$ & $\begin{array}{l}69 \\
69\end{array}$ & $\begin{array}{l}0.0895 \\
0.1345\end{array}$ & $\begin{array}{l}77 \\
77\end{array}$ & $\begin{array}{l}1.8 \\
1.3\end{array}$ \\
\hline 52 & $\begin{array}{l}\text { A } \\
B\end{array}$ & $\begin{array}{l}0.1079 \\
0.0987\end{array}$ & $\begin{array}{l}69 \\
69\end{array}$ & $\begin{array}{l}0.1082 \\
0.0993\end{array}$ & $\begin{array}{l}68 \\
68\end{array}$ & $\begin{array}{l}0.0 \\
0.0\end{array}$ \\
\hline $6 A$ & $\begin{array}{l}\text { A } \\
\text { B }\end{array}$ & $\begin{array}{l}0.0961 \\
0.1080\end{array}$ & $\begin{array}{l}70 \\
60\end{array}$ & $\begin{array}{l}0.1050 \\
0.1050\end{array}$ & $\begin{array}{l}78 \\
68\end{array}$ & $\begin{array}{l}3.0^{\star} \\
0.6\end{array}$ \\
\hline $7 A$ & $\begin{array}{l}\text { A } \\
\text { B }\end{array}$ & $\begin{array}{l}0.0439 \\
0.0856\end{array}$ & $\begin{array}{l}60 \\
69\end{array}$ & $\begin{array}{l}0.0452 \\
0.0819\end{array}$ & $\begin{array}{l}68 \\
77\end{array}$ & $\begin{array}{l}1.5 \\
0.2\end{array}$ \\
\hline $7 \mathrm{C}$ & $\begin{array}{l}\text { A } \\
B\end{array}$ & $\begin{array}{l}0.0941 \\
0.1149\end{array}$ & $\begin{array}{l}60 \\
60\end{array}$ & $\begin{array}{l}0.0917 \\
0.1102\end{array}$ & $\begin{array}{l}68 \\
68\end{array}$ & $\begin{array}{l}0.6 \\
0.3\end{array}$ \\
\hline $7 I$ & $\begin{array}{l}\text { A } \\
\text { B }\end{array}$ & $\begin{array}{l}0.0524 \\
0.0427\end{array}$ & $\begin{array}{l}59 \\
60\end{array}$ & $\begin{array}{l}0.0543 \\
0.0457\end{array}$ & $\begin{array}{l}67 \\
68\end{array}$ & $\begin{array}{l}1.6 \\
2.2\end{array}$ \\
\hline $8 I$ & $\begin{array}{l}\text { A } \\
B\end{array}$ & $\begin{array}{l}0.0868 \\
0.0844\end{array}$ & $\begin{array}{l}50 \\
50\end{array}$ & $\begin{array}{l}0.0898 \\
0.0887\end{array}$ & $\begin{array}{l}58 \\
58\end{array}$ & $\begin{array}{l}1.5 \\
1.8\end{array}$ \\
\hline $8 P$ & $\begin{array}{l}\text { A } \\
\text { B }\end{array}$ & $\begin{array}{l}0.0632 \\
0.0701\end{array}$ & $\begin{array}{l}47 \\
50\end{array}$ & $\begin{array}{l}0.0602 \\
0.0658\end{array}$ & $\begin{array}{l}55 \\
58\end{array}$ & $\begin{array}{l}0.4 \\
0.1\end{array}$ \\
\hline $9 \mathrm{~V}$ & $\begin{array}{l}\mathrm{A} \\
\mathrm{B}\end{array}$ & $\begin{array}{l}0.0246 \\
0.0363\end{array}$ & $\begin{array}{l}40 \\
40\end{array}$ & $\begin{array}{l}0.0298 \\
0.0366\end{array}$ & $\begin{array}{l}48 \\
48\end{array}$ & $\begin{array}{l}3.8 * \\
1.1\end{array}$ \\
\hline $9 w$ & $\begin{array}{l}\mathrm{A} \\
\mathrm{B}\end{array}$ & $\begin{array}{l}0.0237 \\
0.0486\end{array}$ & $\begin{array}{l}10 \\
10\end{array}$ & $\begin{array}{l}0.0259 \\
0.0386\end{array}$ & $\begin{array}{l}18 \\
18\end{array}$ & $\begin{array}{l}1.4 \\
0.2\end{array}$ \\
\hline $10 I$ & $\begin{array}{l}\mathrm{A} \\
\mathrm{B}\end{array}$ & $\begin{array}{l}0.0302 \\
0.0521\end{array}$ & $\begin{array}{l}70 \\
69\end{array}$ & $\begin{array}{l}0.0413 \\
0.0510\end{array}$ & $\begin{array}{l}78 \\
77\end{array}$ & $\begin{array}{l}9.5^{*} \\
0.6\end{array}$ \\
\hline $11 \mathrm{~F}$ & $\begin{array}{l}\mathrm{A} \\
\mathrm{B}\end{array}$ & $\begin{array}{l}0.0838 \\
0.0934\end{array}$ & $\begin{array}{l}70 \\
70\end{array}$ & $\begin{array}{l}0.0960 \\
0.0985\end{array}$ & $\begin{array}{l}78 \\
78\end{array}$ & $\begin{array}{l}4.0 \text { * } \\
2.1\end{array}$ \\
\hline $11 I$ & $\begin{array}{l}\mathrm{A} \\
\mathrm{B}\end{array}$ & $\begin{array}{l}0.0770 \\
0.0734\end{array}$ & $\begin{array}{l}70 \\
70\end{array}$ & $\begin{array}{l}0.0755 \\
0.0745\end{array}$ & $\begin{array}{l}78 \\
78\end{array}$ & $\begin{array}{l}0.6 \\
1.3\end{array}$ \\
\hline $13 \mathrm{C}$ & $\begin{array}{l}\mathrm{A} \\
\mathrm{B}\end{array}$ & $\begin{array}{l}0.0389 \\
0.1743\end{array}$ & $\begin{array}{l}60 \\
70\end{array}$ & $\begin{array}{l}0.0395 \\
0.1675\end{array}$ & $\begin{array}{l}68 \\
78\end{array}$ & $\begin{array}{l}1.3 \\
0.3\end{array}$ \\
\hline $13 I$ & $\begin{array}{l}\mathrm{A} \\
\mathrm{B}\end{array}$ & $\begin{array}{l}0.0355 \\
0.0373\end{array}$ & $\begin{array}{l}69 \\
70\end{array}$ & $\begin{array}{l}0.0382 \\
0.0485\end{array}$ & $\begin{array}{l}77 \\
78\end{array}$ & $\begin{array}{l}2.5 \\
7.6 \text { * }\end{array}$ \\
\hline $14 C$ & $\begin{array}{l}\text { A } \\
\text { B }\end{array}$ & $\begin{array}{l}0.0835 \\
0.1127\end{array}$ & $\begin{array}{l}70 \\
70\end{array}$ & $\begin{array}{l}0.0835 \\
0.1111\end{array}$ & $\begin{array}{l}78 \\
78\end{array}$ & $\begin{array}{l}1.0 \\
0.7\end{array}$ \\
\hline $15 \mathrm{~B}$ & $\begin{array}{l}\mathrm{A} \\
\mathrm{B}\end{array}$ & $\begin{array}{l}0.0669 \\
0.1019\end{array}$ & $\begin{array}{l}40 \\
40\end{array}$ & $\begin{array}{l}0.0666 \\
0.0956\end{array}$ & $\begin{array}{l}48 \\
48\end{array}$ & $\begin{array}{l}0.9 \\
0.3\end{array}$ \\
\hline
\end{tabular}

Outliers are excluded from measurement data.

* Significant at the 0.99 probability level. 
Table 11 - Frequency of Differences Between Participant's Linewidth Measurements and NBS Values for Two Operators on Each of Three Measurements Systems ${ }^{\dagger}$

(a) Row C (opaque lines)

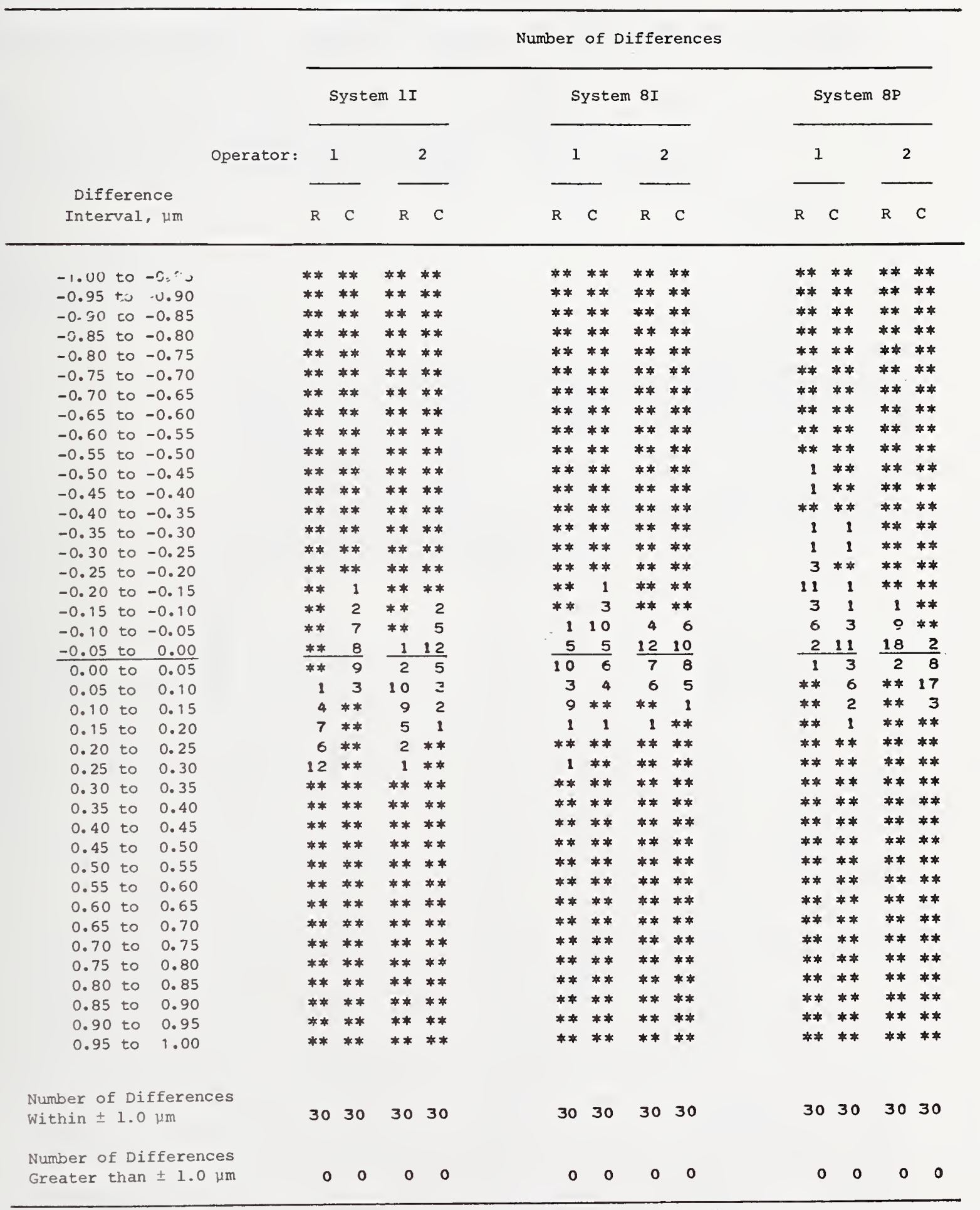

+ The left column (R) for each operator contains the differences for the raw data, and the right column (C) contains the differences for the corrected data. The same two operators did not make the measurements on all three systems. A double asterisk denotes the absence of a difference value. 
Table 11 (Concluded)

(b) Row D (clear lines)

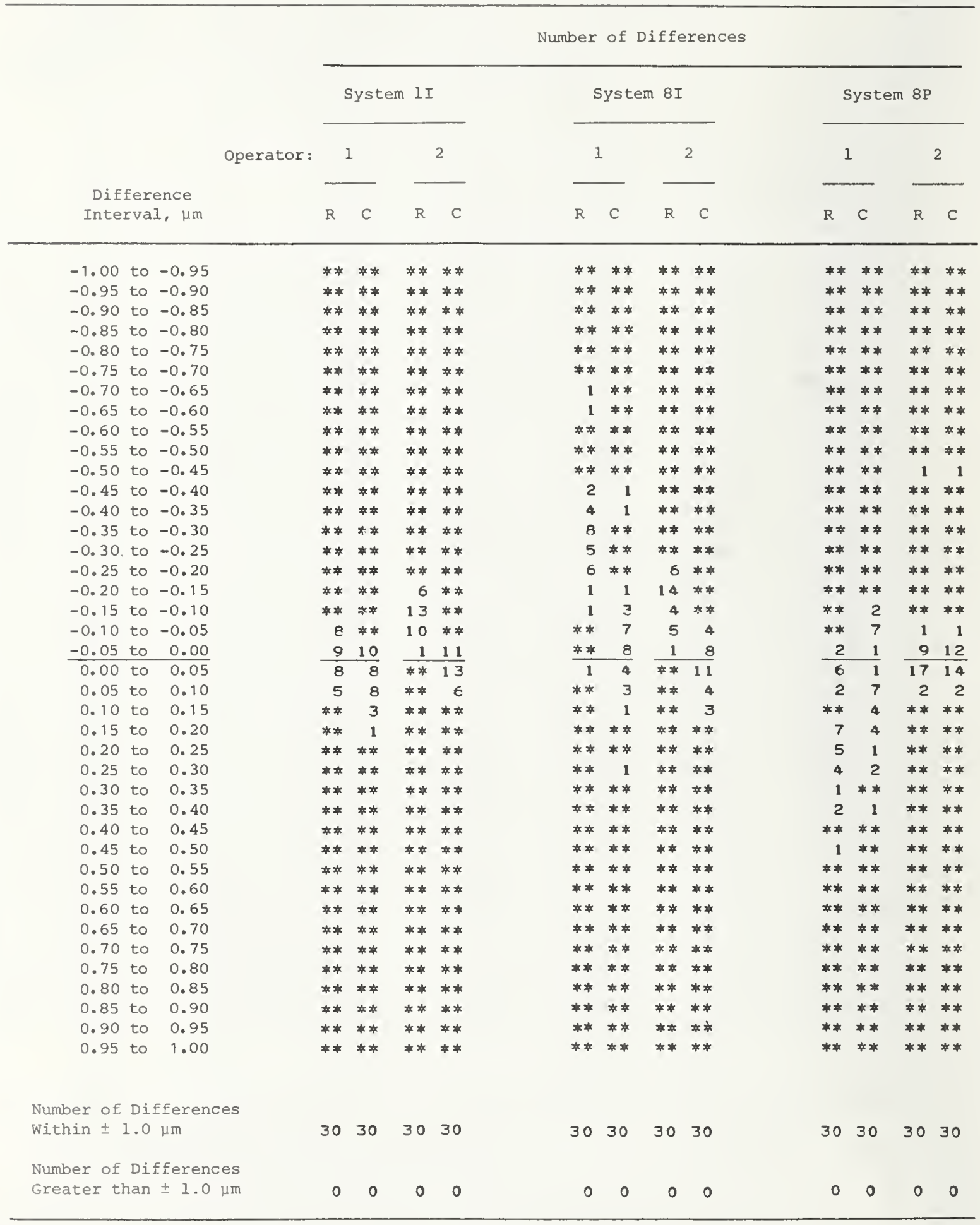




\title{
Design of a Pattern on a
}

\section{Photomask-Like Physical Standard for Evaluation and Calibration of Linewidth-Measuring Systems*}

\author{
Dennis A. Swyt
}

National Bureau of Standards

Optics and Micrometrology Section Washington, D.C.

\begin{abstract}
A pattern for a photomask-llke physical standard, having among its eiements lines of widths and spacings in the 1-to-10 micrometer $\left({ }^{\mu} \mathrm{m}\right)$ range, has been developed to be used by the integrated circuit industry to calibrate lirewidth-measuring optical microscopes. In order to meet the special requirements of a National Bureau of Standards (NBS) measurement transfer program, the pattern has been designed with some essential metrological and statistical principles in mind. The resulting unusual and functionally important features are intended first, to test the ability of an optical system to perform proper linewidth measurements; second, to expose or counteract instrument and operator biases in the measurement process; and third, to allow a transfer of linewidth calibration values with a built-in test of the accuracy and precision of the transfer. The basic pattern can be scaled to micrometer and submicrometer sizes, formed on transmitting photomasks and opaque substrates, and used with linewidth-measuring devices of imaging or non-Imaging, reflecting or transmitting, optical or electron types.
\end{abstract}

Introduction

$A^{\mathrm{T}}$ STAGES IN THE MANUFACTURE of integrated circuit devices during which measurements of the smallest circuit elements on photomasks, substrates-in-process, and finished devices are made, linewidth measurement problems are often acute. These can be problems of repeatability (precision) within individual production houses, or they can be problems of agreement (accuracy) between producers of masks or devices and their consumers. ${ }^{1}$

For example, a mask maker, using a commercial microscope calibrated with a line scale, measures the width of a specific photomask line, obtaining a result which is clearly within specification. Using a similar microscope calibrated with the identical linescale, the IC manufacturer buying the mask measures a width for the same line which is clearly outside specification.

Measurement problems such as this, characterized by serious discrepancies in numerical results, occur when the measurements are not, in a special sense of the word, "proper." The term proper can be used to describe both the numerical result of a measurement process and the process itself. Referring to the numerical result, a proper measurement is one which, by definition, results when the prescribed operations which constitute a measure-

- This effort was conducted as part of the Micrometrology Program and of the Semiconductor Technology Program at the National Bureau of Standards and was funded by the National Bureau of Standards and by the Defense Advanced Research Projects Agency Order 2397. Contribution of the National Bureau of Standards and not subject to copyright. ment process satisfy certain essential conditions. Referring to the process, a proper measurement is one which, because it fulfills certain conditions, predictably yields (within a known uncertainty) a number which is characteristic of a given system; furthermore, the process yields that number whenever and wherever it is performed on the system. ${ }^{2,3}$

There are rules (axioms) which must be operationally defined if proper measurements are to be made. Within the philosophical framework described by Rudolf Carnap, ${ }^{2}$ five such rules in turn define a logically complete measurement system of the type appropriate to linewidths. The means by which these five axioms are to be defined is a prescription which tells in detail how to make the measurements. At present, however, there is in industry, no generally accepted, empirically validated prescription for making proper measurements of the property "photomask linewidth."

One effect of this absence is reflected in the example of the mask maker and his customer cited above; they tacitly and erroneously assumed that proper calibration for linespacings would lead to proper measurement of linewidths. Linewidths and linespacings are, however, separate and distinct measurement properties, each requiring its own valid prescription for proper measurement.

Now a National Bureau of Standards (NBS) program ${ }^{4}$ has generated a prescription, which by specifying equipment and procedures, operationally defines the measurement property "photomask linewidth." The prescription defines the linewidth in terms of the distance between 
two well-defined points in a microscope image of the material photomask line; these two points are at one level on an intensity profile of the line's optical (or electron) image.

Some of the elements in the prescription derive from the logical necessity to define the five rules, some derive from the nature of the physical phenomena of optical or electron imagery, some derive from convention. Detailed specifications cover, for example: an optical system's imaging quality, resolving power, numerical apertures, focus settings, illumination type and wavelength; as well as a detection system's calibration, linearity and threshold or, for a visual microscope, the eyepiece configuration and optical performance. ${ }^{5}$

Such a prescription, however, is not in itself a complete solution to the linewidth measurement problem. Measurements should not only be proper but should be demonstrated to be so. A completely specified measurement system should contain, then, a second important element: procedures to statistically determine how well the necessary axioms have been realized at the level at which the measurements are made. Procedures such as these make it possible to judge the statistical significance of differing measurements, such as those the mask maker encounters. Recognition of the importance of such procedures has lead to the formulation by workers at NBS of a "measurement assurance" approach to calibrations and measurements. ${ }^{\circ}$

The purpose of this paper, then, is to describe a pattern for a photomask linewidth standard which is designed to be a means to measurements which are both proper and demonstrably so. This linewidth standard, upon direct calibration by NBS and subsequent use with NBS-prescribed procedures and instrument parameters. will allow the evaluation and transfer calibration of optical systems for the proper measurements of photomask linewidths.

The paper is divided into four parts: one describes briefly the pattern as it appears on the photomask-like standard; the second discusses the axioms of Carnap which formed the conceptual foundation for the general design; the third discusses the "measurement assurance" principles which formed the basis for the aetailed design; and the last major section of this paper describes the configuration and dimensions of the design in the context of the thinking that went into it.

\section{The Basic Pattem}

The pattern described in this report is designed to be formed on a substrate by conventional IC artwork generation and lithographic transfer. The finished artifact, after subsequent calibration, is to be used as a photomask linewidth standard by the microelectronics industry in its mask metrology. In this paper, the pattern will be described in its application to optical microscopes measuring 1 to 10 micrometers $(\mu \mathrm{m})$ wide lines on light-transmitting photomasks. However, the pattern dimensions can be scaled to sub-micrometer sizes, it can be formed on opaque substrates, and it can be used to

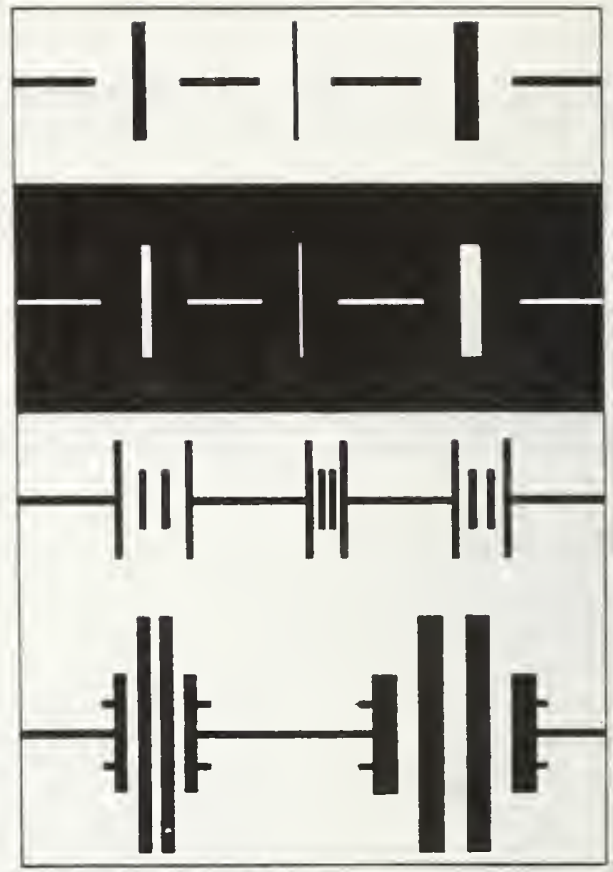

Fig. 1-Elements of a photomask pattern for the evaluation and calibration of linewidth-measuring optical microscopes: (top row) isolated opaque lines and (second row) isolated clear spaces for linewidth measurements; (third row) bracketed line pairs for spacing measurements; and (bottom row) combined isolated and bracketed pairs for image polarity. proximity effect studies.

characterize linewidth-measuring devices of the imaging or non-imaging, reflecting or transmitting, optical or electron types.

There are three major types of features within the pattern, the first two being of most direct importance to the user of the calibrated target. In Figure 1 are shown the three types of features: isolated lines, both opaque on clear fields and clear on opaque fields; linespacing pairs; and bracketed-unbracketed pairs. Figure 2 shows the individual elements of the pattern arranged within the grid of a $6 \times 10$ array, the six rows being lettered $A$ through $F$ and the ten columns being numbered zero through 9. Figure 3 shows characteristic dimensions of these individual pattern elements.

Rows A through D contain forty isolated lines whose individual widths, in the range 1 to $11 \mu \mathrm{m}$, are their most important dimension. The lines are grouped ten per horizontal row of like-polarity: rows $A$ and $C$ contain opaque lines on clear fields, while rows $B$ and D contain clear spaces on opaque fields.

Although there are forty lines in the pattern, there are only twenty different linewidths. The group in Row $B$ is specified to contain the same nominal widths as the group in Row A, although in reversed order; in a like manner, the group in Row D is specified to contain the same nominal widths as the group in Row C. 
Row $E$ contains ten pairs of lines of equal widths whose center-to-center spacings (pitch), also in the range 1 to $11 \mathrm{\mu m}$, are their most important dimension. Each pair of lines is bracketed by another pair, such that the three spaces deined by the four lines in a group are equal in width. The lines themselves are equal in width, their values being $1.0,1.5$, or $2.0 \mu \mathrm{m}$.

Row F contains five pairs of opaque lines, each partially bracketed by another pair, where the nominal equality of the lines and spaces is the most important feature of the pattern element. The characteristic widths of the individual elements are $1,2,3,5$, and $10 \mu \mathrm{m}$, respectively.

\section{Philosphicai Framowork of the Unewidth Pattern Design}

The essential feature of a linewidth standard is some number of lines which can act as "knowns" in a calibration scheme. The pattern described has that feature and more. Much of what is included in the variety of elements on the pattern is there to provide a means to attaining proper measurements.

According to Carnap, ${ }^{2}$ there is a minimum number of logical axioms which must be operationally defined in order to obtain proper measurements of a specific quantity; the number of axioms required depends on the nature of the physical system.

For an extensive quantity (that is, one for which a satisfactory addition or "joining" operator can be defined), three axioms are needed: one axiom defines an equality operator by which one measured quantity can be said to equal another; a second axiom defines an ordering operator by which one measured quantity can be said to be greater or less than another; and a third defines the dimensional unit of the quantity to be measured. For an intensive quantity (that is, one for which no satisfactory addition operator can be defined), five axioms are needed. To the first three are ajded a fourth which defines a zero, either explicitly or implicity, and a fifth axiom which defines a way to generate a continuous scale by which to interpolate between the unit of the third axiom and the zero of the fourth. This "zero" can be interpreted as a second unit since it may have a mathematically non-zero value.

Since the concept of "linewidth" may be a somewhat unfamiliar one to some readers, the meaning of each axiom and their relationships to each other will be discussed first in terms of the more familiar quantities. mass and temperature.

The first two of the axioms, those of the equality operator and of the ordering operator, taken together determine "what" is being measured by defining "how" to measure it.

For example, what is meant by the property of matter "mass" depends on whether one uses a pan balance and Newton's law of universal gravitation or an "inertial balance" and Newton's second law of motion to say that "this body has greater, less or the same amount of mass as that body." Operationally defined by these two means. gravitational mass and inertial mass can be two inde-

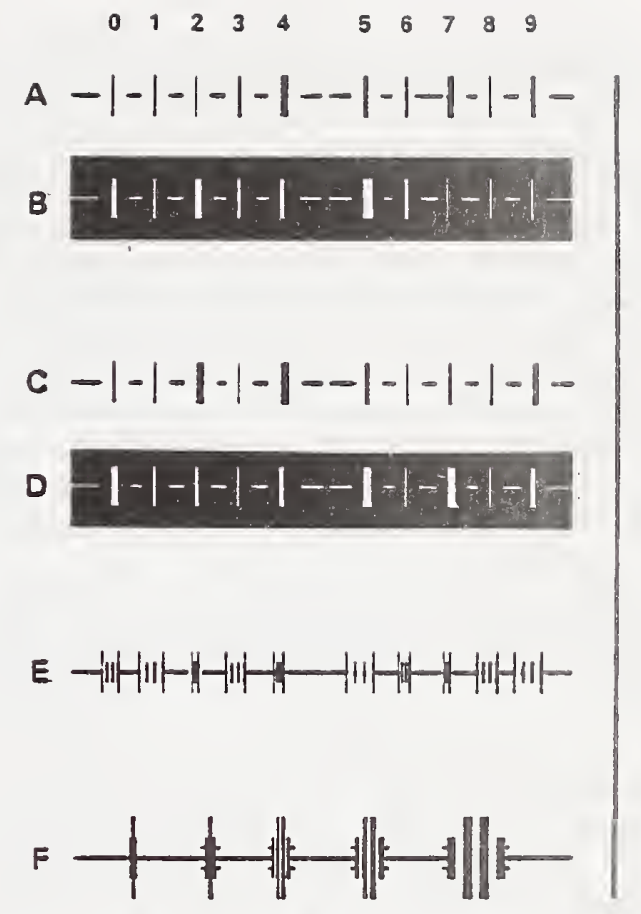

Fig. 2-Scale drawing of linewidth-artifact pattern incorporating basic elements: Rows $A$ and $C$-isolated clear lines on opaque field; Rows $B$ and $C$-isolated opaque lines on clear field. Row $E$-bracketed line pairs of various spacings; Row F-combined bracketed and isolated pairs.

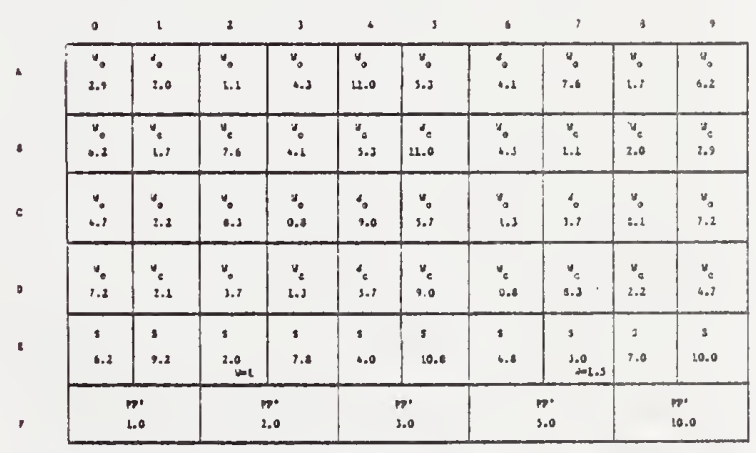

Fig. 3-Map of linewidth artifact pattern showing (in dimensions of $\mu \mathrm{m})$ : the widths of opaque $\left(W_{0}\right)$ and clear $\left(W_{e}\right)$ isolated lines for linewidith measurements; the spacings of bracketed line pairs (S) for linespacing measurements: and the widths of lines and spaces in combination bracketed $(P)$ and isolated $\left(P^{\prime}\right)$ pairs for image polarity-proximity effect studies. Please note that the linewidth values quoted, while representative of, are not equal to those on the physical standard since it must act as an "unknown" in a current NBS-industry study.

pendently proper measurement properties of the same body.

The other three axioms, those of the unit, the zero. and the scale, taken together determine how numerical values are assigned to observed states of the property which is being measured. 
For example, temperature is a property which requires the operational definition of these additional three axioms for its proper measurement. One way to convert the subjective impressions of hotter, colder, same "temperature," into objective observations is by using a liquid-in-glass tube thermometer as the equality-ordering operators. Then the height of the liquid column when the thermometer is in contact with boiling water can define a unit (212 degrees), similarly the height of the column when the thermometer is in contact with freezing water can define a "zero" (32 degrees). And the prescription for forming the bore in the tube and ruling the graduations can define the means for dividing the numerical difference between the unit (212) and the zero (32) into 180 equal divisions.

Like temperature, the measurement quantity "photomask linewidth" is an intensive one; the prescription telling how to perform the measurement must operationally define five axioms. Specifically, this prescription must tell what things to manipulate and what actions to perform in order to give meaning to the abstract idea: "the width of a material line on a photomask is the perpendicular distance between its two (parallel) edges." The NBS prescription defines this width as the distance between the two points at a certain intensity level on the intensity profile of the optical (or electron) microscope image of that photomask line, when the microscope itself meets certain requirements and is operated under specified conditions. ${ }^{5}$

The most basic function of a prescription such as this one is to define the "what" of the measurements, photomask linewidth, by first of all defining the ordering and equality axioms. Errors arising from absent, incomplete or faulty definitions of these two axjoms are not difficult to observe. For example, there is evidence that the commonly used linespacing-calibrated filar and image-shearing eyepieces can perform ordering-equality operations with opposite results: a flar showing line $\mathrm{A}$ wider than line $\mathrm{B}$, an image-shearing showing line $\mathrm{B}$ wider than line A. ${ }^{8}$ Other types of photomask linewidth measurement errors occur because of the faulty and incomplete realization of unit, zero and scale-generation axioms.

Figure 4 shows the results of different types of pure and combination axiom errors. In a proper measurement system the observed values are correct at all points along a linear interpolation between the defined values of the unit and the zero. Pure errors in either the unit (Figure $4 a$ ) or the zero (Figure $4 b$ ) yield observed values which are correct at one end of the range but in maximum error at the other end. A scale-generation error gives correct observed values at the unit and zero values, but deviations of some type between them (Figs. $4 c$ and $4 f$ ).

Experience at NBS with optical-microscope linewidth measurements, both empirical and analytical, 4,8 leads to the expectation that a common form of error in the measurement of photomask linewidths can be described by Fig. 5. In region I, the dominant error is the unit-zero combination of the incremental type; not shown, but
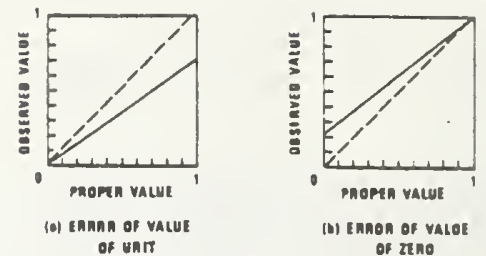

(1)) Enดen of vato:

of ztho

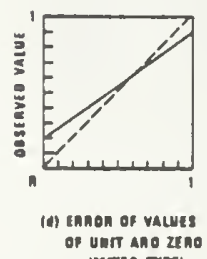

(mole nivi)
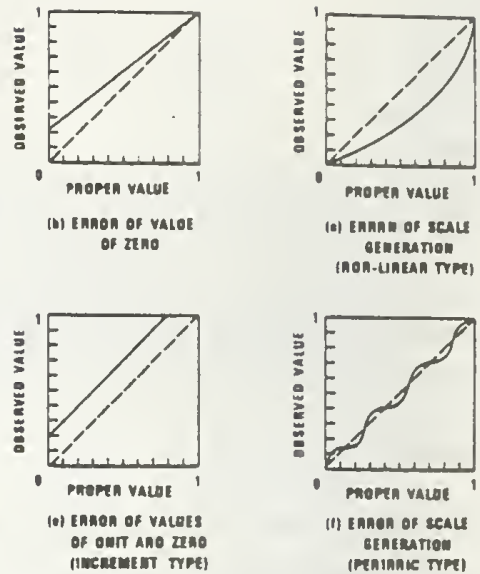

(e) (nang of scau thman of real etwrungen

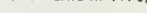

Fig. 4-Examples of types of fundamental measurement errors within five-axiom scheme for extensive physical quantities described by Rudolf Carnaps. Pure forms of errors in: $(A)$ realized value of unit; $(B)$ realized value of zero; $(C)$ and $(F)$, continuously generated scale; and mixed forms of errors in realized values of unit and zero of (D) general and $(E)$ special, incremental types.

likely to be present, is some degree of scale-generation error of either (or both) the non-linear or periodic types. In region $\Pi$, a strong scale-generation error, which arises as the resolution limit of the measuring instrument is approached, is linked with zero and unit errors.

At the industrial user's level, these unit-zero errors can occur because the required prescription for measurement, conventionally accepted and based on the physics of image formation, is absent. Due to this absence, the unit, zero and scale generation of a completely different physical quantity, line-spacing, are usually carried over tacitly to linewidth measurements. While the scale generation scheme can be carried over, the unit and zero fail; these failures occur because the ordering equality operators for linespacing and linewidth differ in a fundamental way.

A measurement of a linespacing is a "symmetric" one, while a measurement of a linewidth is "asymmetric." Linespacings are measured center-to-center, left-edgeto-left-edge, or right-edge-to-right-edge of two different lines. The "handedness" of the detection at each line is the same; hence, "symmetric." Linewidths, however, are measured left-edge-to-right-edge of the same line; the "handedness" of the detection at one edge is the opposite of the handedness at the other edge; hence, "asymmetric."

The effect of the presence or absence of symmetry in the measurement can be described in the language of optics. If $x$ represents the location of a line's edge in object space and $y$ represents the location of the same line edge in image space, for a microscope which linearly maps the object into the image, then:

$$
y=a x+b
$$

A linespacing meac--rement with such a system is the 
difference of two $y$ coordinates which, because the detector in effect approaches each of two lines from the same direction, have the same origin:

$$
\begin{aligned}
S(\text { spacing })=y_{2 R}-y_{2 R} & =\left[a x_{1}+b_{R}\right]-\left[a x_{2}+b_{R}\right] \\
& =a\left[x_{1}-x_{2}\right] .
\end{aligned}
$$

Since the two intercepts, $b_{R}$, are of the same "handedness," they cancel. A linewidth measurement with the same system, however, is the difference of two $y$ coordinates which, because the detector in effect approaches the two sides of the same line from opposite directions, have different origins:

$$
\begin{aligned}
W(\text { width })=y_{1 H}-y_{l t} & =\left[a x_{1}+b_{R}\right]-\left[a x_{2}+b_{L}\right] \\
& =a\left[x_{1}-x_{q}\right]+\left[b_{R}-b_{L}\right] .
\end{aligned}
$$

The intercepts, $b_{R}$ and $b_{L}$, being of different "handedness," do not cancel. This equation exemplifies the form of the unit-zero error of the incremental type shown in Fig. $4 \mathrm{E}$ and region I of Fig. 5.

Phenomenologically, the $b$ intercepts can be interpreted as image "edge shifts" which prevent one-to-one correspondence of object and image dimensions. They are complex functions of, for example, the imaging system's optics and illumination, the object polarity (clear or opaque), the illumination polarity (reflection or transmission), and the detailed operation of the measuring device (visual filar or image-shearing eyepiece, CRT scan, etc). These "edge shifts" and their resulting offsettype errors are not "constants" but vary among instruments, among operators on the same instrument (especially visual eyepiece systems), and between times on the same instrument-operator system. ${ }^{8}$

Even with explicit "edge shift" effects present, there are, however, no scale-generation errors in the example cited above; and the same scale applies to both the linespacing and linewidth measurement. The test of a scalegeneration operation is that intervals in different regions along the scale are equal. Formally, this is stated as: For the line spacings, if certain conditions are met,

$$
S_{1}-S_{2}=S_{3}-S_{2}:
$$

For the linewidths, if certain conditions are met,

$$
W_{1}-W_{2}=W_{1}-W_{4}
$$

On inspection of equations (2) and (4), it can be seen that the difference between two linewidths is free of the "handedness" constants and has only the multiplicative constants of the linespacings. Therefore, the pitch of a micrometer screw or the linear timebase of a CRT sweep can be used to generate a scale for either linespacing or linewidth measurements; however, without a proper realization of their own unit and zero, linewidth measurements cannot be made correctly on a linespacing-calibrated device.

The fact that linespacing and linewidth measurements can naturally share a scale-generation operation has led to one of the design features of the pattern. Since line-

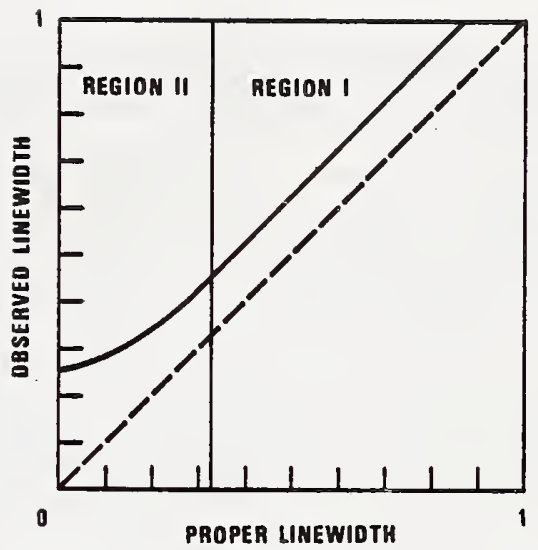

Fig. 5-Forms of errors anticipated to be encountered in industrial photomask linewidth measurements for linewidths below $10 \mu \mathrm{m}$; in Region 1 , the combined unit-zero error of the incremental type; in Region II, the combined unit-zeroscale generation error.

spacing measurements can, in general, be done more precisely than linewidth measurements, a spectrum of linespacings has been included in the pattern to test the ability of the operator-instrument system to generate a proper linewidth scale.

Application of Measurement Assurance Princlples to the Design

The previous section has dealt with the requirements which must be satisfied for measurements to be proper. Also discussed were the types of errors which arise if these requirements are not met. This section will deal with a technique for determining whether or not a functioning measurement system is yielding proper measurements by looking for error.

The technique, developed at NBS with contributions by a number of workers, is called "measurement assurance". 6.7 Its formulation was guided by an analogy between manufacturing processes (which generate product) and measurement processes (which generate numbers). By the analogy, quality assurance procedures apply to both processes. A manufacturing process is considered to be in "quality control" if the product being generated is within tolerances; similarly, a measurement process is considered to be in "statistical control" if the numbers generated and their variations lie within acceptable limits. This statistical control is a sign that the measurements are proper, a demonstration that in fact the necessary axioms have been realized.

For many of the most economically important measurements in an industrial society, such as the accept-orreject linewidth measurements on the mask maker's product, two complete sets of axioms must be realized and tested, one set at each of two levels or stages of the measurement process.

At the first stage are the direct or primary measurements. These yield numbers from a fundamental realization of the defined property, such as linewidth values 
which are derived from the analysis of a properly formed image profile. Such measurements can be complex and expensive to make. At the second stage are the transfer or secondary measurements. These yield numbers from a comparison of an unknown with a known, such as linewidth values which are derived from a comparison of lines of unknown widths with lines for which widths have been measured directly.

The added complexity of this two-stage process is justified when the comparison-transfer measurement is significantly easier and more economical than the direct-calibration measurement. In the real world, this is usually the case; for many smaller manufacturing firms. transfer measurements are the only ones practically attainable.

In the context of this paper, the two levels of the process are the calibration stage which takes place in an NBS laboratory and the transfer stage which takes place in an industrial plant. The pattern described here has been tailored to meet the special needs of this system.

In particular, a sufficient number of elements of the right kind have been included in the pattern to make possible a statistically-engineered test of the basic axioms of linewidth measurement. With a calibrated physical standard bearing such a pattern in hand, a user can follow prescribed directions, perform repeated measurements and obtain a well-defined curve of observed-versusproper (calibrated) linewidth values, a curve such as one of those in Figs. 4 or 5. This curve can be analyzed for slope, intercept and deviations from linearity to determine the errors present; both fundamental axiom errors as well as "random" scatter errors.

Since the goal of the program for which the pattern was designed is to attain accurate linewidth measurements at the industrial user's level, a collaborative interlaboratory study of linewidth measurements is being made between individual microelectronics industry firms and NBS. ${ }^{\circ}$ What follows is a sketch of the manner in which the pattern-bearing physical standard is to be used in that study.

With the pattern formed by conventional lithography on a substrate, linewidths and linespacings on the resulting photomask-like object are to be measured at NBS.

Some or all, as deemed necessary, of the linewidths are to be measured by a combination of electron microscope/photometric optical microscope procedures; these measurements constitute a fundamental realization of the physical quantity "photomask linewidth". Some or all of the linespacings, again as deemed necessary, are to be calibrated on the NBS linespacing interferometer. ${ }^{1 n}$

The physical standard, bearing lines of calibrated widths, is then supplied to a user within the microelectronics industry.

Designated linewidths and linespacings on the calibrated standard are to be measured by such participants in the study without, however, their having knowledge of the calibration values. The procedure for the measure- ments is prescribed by NBS; included in it are details of the instrument set-up, the specific lines to measure, the sequence in which to measure them, and the number of repeated measurements.

Completed data sheets and the physical standard are then returned to NBS.

In broad terms, analysis of the data performed by NBS is to determine how well the necessary axioms are realized at the NBS-calibration stage and at the usertransfer stage of the two-stage measurement process. In more specific terms, the data will be used to assess: (1) the integrity and completeness of the prescribed procedure, (2) the suitability of the calibrated artifact standard, and (3) the precision and accuracy attainable and attained by the user of the physical standard.

At present, one of the working assumptions of the experiment is that, since most linewidth measuring instruments operate as comparators, the common linewidth error of Fig. 4 (Region I) can be eliminated by proper two-point calibration. Deviations from this expected behavior in the results of the study of the measurement process are to be judged within the "model ambiguityalgorithm error-unit error" scheme formulated among Cameron, Eisenhart, Pontius and Simpson and described by Pontius. ${ }^{6}$

Within this scheme, the measurement system is considered to include these elements: (1) an object-which is the physical thing with a property to be measured; (2) a model-which is the conception of the object and its properties; (3) an algorithm-which is the instrumentation, manipulative procedures, computations, etc, taken together; and (4) a unit-which is the defined value of measurable quantity, having zero error by definition.

From the inter-relationships of these elements originate three sources of error, two more important than the third. "Model ambiguity" causes "perfect" measurements to disagree because the real object behaves differently than the conceptual model predicts. "Algorithm error" causes measurements of "perfect" objects to disagree because the measurements are not proper. "Unit error," the least significant error of the three, is the disagreement between the unit as locally realized and the unit as expresed by the assigned number (e.g., the number assigned the international SI unit of length).

Besides providing a framework for error analysis, the model-algorithm concept has influenced the specification for manufacture of the physical standard being discussed. In order to focus on algorithm errors by reducing model ambiguity to a minimum, the object is to be refined to fit the existing conceptual model. In practice, this means that the physical standard will be "idealized," having properties such as line straightness and edge acuity more akin to best-effort output of the industry than to the typical photomasks to be measured.

To the extent that the standard is "idealized" and used for this initial study outside the stream of everyday production measurements, the study contains deviations from an end-form which is being sought. 


\section{Configuration and Dimensions of Elements}

Apart from the general design considerations discussed, certain more specific "measurement assurance" principles have been applied in the design of the pattern. One such principle is that the objects to be measured should have attributes in themselves which tend to counteract and eliminate biases and errors in the measurement process. Application of this principle has determined how the lines of measured widths and the lines of measured spacings are grouped, how the lines of different widths and spacings are located relative to each other within groups, how the values of widths and spacings are distributed over the range of values, and finally. how the linespacing pairs are bracketed.

\section{The Linewidth Groups}

The range of linewidths ( 1 to $11 \mu \mathrm{m}$ ) included in the pattern covers the region in which the measurement problems addressed by the NBS photomask program lie.* Within this range, the number, distribution, and sequence of linewidths in the pattern have been chosen for statistical purposes.

In order to adequately cover the range of interest, ten lines of various widths, distributed over the range, were chosen for a single group. Ten other lines of the same polarity (opaque or clear) in a distribution of similar but slightly different values of linewidth became a second group. Inclusion of two distinct groups allows one group to be treated as a calibration or control group and the other as an unknown in an approximation of an instrument calibration-photomask inspection process. See, for example, Rows $A$ and $C$ of Figs. 2 and 3. either of which may be the control for that polarity.

Both of the distributions were derived from a single, starting distribution of linewidths (in $\mu \mathrm{m}$ ): 1.0, 1.5, $2.0,2.5,3.5,4.5,5.5,7.0,8.5,10.0$. The important properties of the basic distribution are: (1) the intervals are non-uniform to avoid the possibility of being in phase with the type of periodic errors shown in Fig. 4F; and (2) the distribution has more points at its low end because of the anticipated behavior of systems shown in region II of Fig. 5.

The two distributions associated with the control and unknown groups were generated from the basic distribution given above by a "coin-toss" incrementing technique. For each linewidth of the basic distribution, an increment of either $0 \%$ or (approximately) $10 \%$ of its value was chosen for it by coin toss; if the non-zero value were indicated, a second coin-toss determined the sign of the increment. In such manner, each linewidth value in the basic distribution was incremented. The entire procedure was then repeated and two distinct but similar distributions obtained.

The presence of two such distributions on one pattern provides a number of pairs of lines: (1) lines which are of like polarity; (2) lines which are similar, sometimes nearly equal but non-identical in width; and (3) lines which are somewhat physically removed from each other. Interromparison of measurements made on respective members of such pairs allows some estimate of the resolution of the ordering-equality operations of the measurement process.

In Fig. 3, an array map of the entire pattern, a simulation of these distributions of linewidth values is given. Actual values are not reported in order to preclude current and future participants in the NBS-industry study from obtaining foreknowledge of what are supposed to be, for them, unknowns.

The order in which lines of various widths appear in the pattern is not one of ascending or descending values of linewidth, but a randomized one. This implied sequence of measurements is to counteract operator bias in the anticipation of values and to prevent the cumulative effects of hysteresis and "memory"-type effects in instruments.

Two minor details of the design of the lines as they appear in rows $A$ through $D$ of Fig. 1 are worth mention: the lines were made long enough to give a moderately high aspect ratio for the widest one; and horizontal orientation lines, run between the isolated lines to locate points half-way down their lengths, are kept far enough away to prevent the sheared images in an imageshearing visual eyepiece from overlapping them.

\section{The Linespacing Group}

Although linespacings are a distinctly different measurement quantity from linewidths, lines of known spacings have a place in the pattern: they can function as a highprecision check of the lin; $*$ idth scale-generation. There are design features of these line pairs which give them special advantages as linespacing standards in themselves. These features are related to principles of symmetry, independence of measurements, disorder, and pattern recognition.

The basic configuration of the linespacing element is shown in the third row of Figure 1 ; it consists of a pair of short lines bracketed by a longer pair. The distance from a point on one of the short lines along a horizontal axis to a similar point on the other short line is the dimension of interest, the line spacing. Since the two short lines are specified to be equal in width, the leftedge-to-left-edge, right-edge-to-right-edge and center-tocenter distances are nominally equal; this distance is the linespacing or pitch.

The linespacing values are 2.0, 3.0, 4.0, 4.8, 6.2, 7.0. $7.8,9.2,10.0$, and 10.8 micrometers. In all the linespacing elements but two, the width of the lines is $2 \mu \mathrm{m}$. The spaces are of various widths. For reasons related to the lithography of photomasks and to imaging phenomena, the two elements having the lowest linespacing values are formed with lines and spaces equal in width; these widths are 1.0 and $1.5 \mu \mathrm{m}$ respectively for these two elements.

The lines of the outer pair bracketing the inner pair are of fundamental importance: they add a necessary symmetry to each line of the inner, linespacing pair. With 
an isolated pair of lines, ones without the bracketing pair, each line has one "interior" and one "exterior" edge. Asymmetries in the material profiles of the lines as well as in the intensity profiles of their optical or electron images can make measurements of their spacing biased. Addition of the bracketing pair, as dummy lines, makes all edges in the linespacing pair "interior" edges. thus eliminating an asymmetry and a potential source of measurement error. The brackets are made longer for two reasons: (1) to differentiate them from the linespacing pair and thus to avoid confusion during measurement (this is especially useful for image-shearing eyepiece measurements where the presence of many lines in the sheared images causes confusion), and (2) to create a clearly identifiable configuration having a "patternrecognition" quality which separates the linespacing group from the one below it on the overall pattern.

The linespacing elements have three other properties usually absent from linescale standards. First, each linespacing value is defined by a single pair of lines which are physically independent of the lines which define other values. Thus, measurements are decoupled from each other: $S_{1}=X_{1}-X_{2}, S_{2}=X_{3}-X_{4}, S_{3}=X_{5}-X_{6}$, not $S_{1}=X_{3}-X_{2}, S_{2}=X_{1}-X_{4}, S_{3}=X_{1}-X_{4}$. Second, the linespacing values are of non-equal intervals; and, third. they occur in a randomized order of relative values. The purposes of the latter two properties have been discussed above in their application to linewidth values.

\section{The Bracketed-Unbracketed Pair Group}

These elements (bottom row, Fig. 1) are designed to have a pair of lines which are bracketed about the central portion of their lengths and unbracketed about their ends. The clear spaces separating the opaque lines within the elements are specified to be equal in width to each other and the lines; the basic dimensions of the group are 1, 2, 3, 5 and 10 micrometers. All horizontal lines attached to the elements are orientation reference marks. The purpose of the elements is primarily to provide, when necessary. information on the imaging properties of the lithography which forms the pattern on the substrate and on the imaging properties of the optical or electron system which views the pattern. These pattern elements may also be used to set the controls of systems such as TV-microscopes which require for calibration an image having known line-to-space ratios.

\section{Conciuslon}

Explicitly, this report describes the thought processes behind the design of a pattern for a photomask-like physical standard for linewidths; implicitly, it describes an attempt to make abstract measurement philosophy pay off in the marketplace.

The thought processes reflect an attempt to apply to a new and evolving measurement system the synthesis of two important measurement ideas: the one which asserts that proper measurements require the operational definition of essential axioms and the other which asserts that proper measurements require demonstration of the realization of those axioms.

The pattern described in this report has been designed, then, not only as a calibration standard, but as a tool to examine the measurement process. Such an examination is about to take place in a limited study.

Because of the design features of the basic pattern, the details of the measurement procedure specified, and the structure of the overall measurement repetition scheme, statistically reliable results on the soundness of the entire measurement process at the user's level are expected.

Such information is important to the user because it can become a means to attaining accurate and precise photomask linewidth measurements on the production line.

\section{Acknowledgements}

The author thanks Dr. J. A. Simpson both for his timely introduction of the author to Carnap's philosophical framework of measurements and for his contribution of ideas to this paper; Mr. Paul Pontius for his counsel on the ineans to serve an end-user by a measurement assurance program; Mr. John Jerke for his labor and planning on the lay-out of a practical photomask pattern; Mr. John Heine for his drafting; and the NBS Office of Measurement Services for guidance in tailoring the pattern to achieve a statistically sound experiment.

\section{References}

I. Jerke, J., "Semiconductor Measurement Technology: Optical and Dimensional Measurement Problems with Photomasking in Microelectronics", NBS Special Publication 400-20, a vailable from U.S. Government Printing Office, Washington, D.C. 20402, DC Catalogue No. C13.10:400-20.

2. Carnap, Rudolf, "Philosophical Foundations of Physics," Basic Books, New York and London, 1966, pp. 51-95.

3. Simpson, J. A., "On the Dialectic of Measurement," Unpublished talk given at the National Bureau of Standards, Mechanics Division Seminar, Nov. 1975.

4. Swyt, D., "NBS Program in Photomask íinewidth Measurements," Solid State Technology, April 1976.

5. Jerke, J. M., Nyyssonen, D., Rosberry, R. W., Scire, F. E., Swing, R. E., Swyt, D. A., and Young, R. D., "Semiconductor Measurement Technology: Accurate Linewidth Measurements on Integrated Circuit Photomasks," NBS Special Publication 400-43, available from U.S. Government Printing Office, Washington, D.C. 20402.

6. Cameron, J. M., “Measurement Assurance," National Bureau of Standards, NBSIR 77-1240, available from U.S. Government Printing Office, Washington, D.C. 20402.

7. Pontius, Paul, "Notes on the Fundamentals of Measurement and Measurement as a.Production Process", National Bureau of Standards, NBSIR. 75-545, available from National Technical Information Service, Springfield, Va. 22151.

8. Swyt, D. A. and Rosberry, F. W., "A Comparison of Some Optical Microscope Measurements of Photomask Linewidths," Solid State Technology, August 1977.

9. Swyt, D. A., Rosberry, F. W., and Nyyssonen, D., "Calibration of Optical Microscopes for Photomask Linewidth Measurements," Talk given at Interface 77, Kodak Seminar, Monterey. California, Oct. 5-7, 1977.

10. Gilliand, K. E., Cook, H. D., Mielenz, K. D. and Stephens, R. B. "Use of a Laser for Length Measurements by Fringe Counting," Metrologia vol. 2, 95-98 (1966). 
The mean values for linewidths and line spacings assigned to each of the nine artifacts used in the interlaboratory study are given in table B-1. Each line of data in this table corresponds to one row of patterns on the artifact; for example, the first line of data corresponds to linewidth values for lines A-0 through A-9 and the fifth line of data corresponds to line spacings for line pairs E-0 through E-9. (See sec. 2.1, fig. 3.) The letters and numbers in the right-hand column are a code used to identify the line of data. The first letter in this code is the row on the artifact, the next three letters identify the data as coming from NBS, the first two numbers identify the artifact, and the last number is the particular pattern group on the artifact that was measured by NBS.

Table B-1 - Mean Values of Linewidths and Line Spacings Assigned to Artifacts by NBS

\begin{tabular}{|c|c|c|c|c|c|c|c|c|c|c|}
\hline & & & & Mean & Values, & $\mu \mathrm{m}$ & & & & Code \\
\hline 2.54 & 1.59 & 0.79 & 4.31 & $10 \cdot 60$ & $5 \cdot 34$ & 3.72 & 7.47 & 1.32 & 6.18 & ANES $52-1$ \\
\hline 6.46 & 1.47 & 7.88 & 3.93 & 5.58 & 10.89 & 4.56 & 0.67 & 2.24 & 2.73 & BNBS52-1 \\
\hline 4.77 & 1.78 & 8.29 & 0.80 & 8.92 & 5.27 & 1.28 & 3.31 & 2.13 & 6.78 & CNESS2-1 \\
\hline 7.05 & 2.33 & 3.54 & 1.45 & 5.60 & 9.35 & 0.92 & $8 \cdot \in 0$ & 2.05 & 4.96 & DNAS52-1 \\
\hline 6.19 & 9.16 & 2.00 & 7.78 & 4.00 & 10.70 & 4.77 & 3.00 & 6.95 & 9.59 & ENOS52-1 \\
\hline 2.48 & 1.91 & 0.72 & 4.24 & 10.51 & 5.27 & 3.67 & 7.42 & $1 \cdot 24$ & $6 \cdot 11$ & ANBS53-3 \\
\hline 6.48 & 1.51 & 7.91 & 3.98 & 5.58 & 10.95 & 4.58 & 0.71 & $2 \cdot 26$ & 2.76 & BNES $53-3$ \\
\hline 4.69 & 1.70 & 8.23 & 0.73 & 8.25 & 5.23 & 1.24 & 3.27 & 2.06 & 6.72 & CNASS3-3 \\
\hline 7.09 & 2.36 & 3.57 & 1.49 & 5.62 & $9 \cdot 37$ & 0.93 & 8.62 & 2.06 & 4.99 & DN8S53-3 \\
\hline 6.16 & 9.15 & 1.97 & 7.76 & 3.98 & 10.75 & 4.75 & 2.97 & 6.95 & 9.97 & ENBS53-3 \\
\hline 2.29 & 1.75 & 0.53 & 4.04 & $10 \cdot 35$ & 5.08 & 3.49 & 7.25 & 1.10 & 5.96 & ANBS $54-1$ \\
\hline 6.77 & 1.79 & 8.18 & 4.24 & $5 \cdot 87$ & $11 \cdot 19$ & 4.85 & 1.04 & 2.52 & 3.03 & ENAS54-1 \\
\hline 4.56 & 1.52 & 0.07 & 0.55 & 8.67 & 5.01 & 1.03 & 3.07 & 1.87 & $6.5 C$ & CNAS54-1 \\
\hline $7 \cdot 31$ & 2.62 & 3.81 & 1.76 & 5.92 & 9.64 & 1.25 & 8.90 & $2 \cdot 34$ & 5.26 & DNES54-1 \\
\hline 6.19 & 9.17 & 1.99 & 7.78 & 4.00 & $10.7 \epsilon$ & 4.78 & 3.00 & 6.95 & 9.99 & ENBS54-1 \\
\hline 2.40 & 1.84 & 0.64 & 4.19 & 10.44 & 5.19 & 3.60 & $7 \cdot 33$ & 1.17 & 6.02 & ANES55-6 \\
\hline 6.54 & 1.58 & 7.95 & 3.98 & 5.66 & 11.01 & 4.65 & 0.70 & $2 \cdot 31$ & 2.85 & BNESS5-6 \\
\hline 4.66 & 1.63 & 8.16 & 0.68 & 8.78 & 5.15 & 1.13 & 3.17 & 1.98 & 6.64 & CNESS5-6 \\
\hline 7.17 & $2 \cdot 44$ & 3.63 & 1.57 & 5.70 & 9.44 & 1.01 & 8.70 & 2.12 & 5.06 & DNBS $55-6$ \\
\hline 6.18 & 9.19 & 1.98 & 7.77 & 3.99 & 10.77 & 4.77 & 2.98 & 6.55 & 9.98 & ENESS5-6 \\
\hline 2.42 & 1.86 & 0.65 & 4.20 & 10.50 & 5.21 & 3.62 & 7.37 & 1.21 & 6.06 & ANBSSE-5 \\
\hline 6.60 & 1.60 & 8.00 & 4.06 & 5.70 & 11.03 & 4.66 & 0.66 & $2 \cdot 36$ & 2.86 & BNESSE-5 \\
\hline 4.64 & 1.63 & 8.16 & 0.68 & 8.77 & 5.15 & 1.17 & 3.19 & 2.02 & 6.65 & CNOSSE-5 \\
\hline 7.18 & 2.46 & $3 \cdot<6$ & 1.57 & 5.73 & 9.45 & 0.98 & 8.73 & $2 \cdot 15$ & 5.09 & DNASS $\epsilon-5$ \\
\hline 6.18 & 9.17 & 2.00 & 7.78 & 4.00 & 10.76 & 4.77 & 3.00 & 6.95 & 9.99 & ENES5 $C-5$ \\
\hline 2.52 & 1.94 & 0.78 & $4 \cdot 32$ & 10.61 & $5 \cdot 33$ & 3.71 & 7.46 & $1 \cdot 32$ & 6.19 & ANBS57-4 \\
\hline 6.51 & 1.50 & 7.91 & 3.94 & $5 \cdot 61$ & 10.94 & 4.59 & 0.62 & $2 \cdot 25$ & 2.75 & 8N8557-4 \\
\hline 4.76 & 1.74 & 8.29 & 0.76 & 8.93 & $5 \cdot 30$ & 1.29 & 3.28 & 2.09 & 6.79 & CNES57-4 \\
\hline 7.08 & $2 \cdot 37$ & 3.55 & 1.46 & 5.62 & $9 \cdot 37$ & 0.89 & 8.63 & 2.05 & 4.97 & DNES57-4 \\
\hline 6.19 & 9.17 & 1.99 & 7.78 & 4.00 & 10.77 & 4.78 & 3.01 & 7.00 & 9.99 & ENBS57-4 \\
\hline 2.45 & 1.89 & 0.69 & 4.22 & 10.55 & 5.25 & 3.65 & 7.41 & 1.24 & 6.12 & AN8S58-3 \\
\hline 6.56 & 1.56 & 7.96 & 4.03 & 5.66 & 10.98 & 4.64 & 0.74 & $2 \cdot 33$ & 2.83 & 8NES5B-3 \\
\hline 4.69 & 1.68 & 8.21 & 0.69 & 8.83 & 5.18 & 1.19 & 3.24 & 2.03 & 6.69 & CN8S58-3 \\
\hline $7 \cdot 12$ & 2.42 & 3.61 & 1.55 & 5.68 & 9.43 & 0.98 & 8.67 & $2 \cdot 11$ & 5.07 & DNAS58-3 \\
\hline 6.18 & 9.17 & 2.00 & 7.77 & 4.00 & 10.78 & 4.77 & 3.00 & 6.99 & 9.99 & ENES58-3 \\
\hline 2.63 & 2.05 & 0.88 & $4 \cdot 39$ & 10.71 & 5.42 & 3.83 & 7.57 & 1.48 & 6.25 & ANESSS-2 \\
\hline 6.37 & 1.39 & 7.77 & 3.84 & 5.48 & 10.79 & 4.48 & 0.62 & $2 \cdot 15$ & 2.68 & 8NBS59-2 \\
\hline 4.88 & 1.88 & 8.40 & 0.90 & 9.02 & $5 \cdot 36$ & 1.41 & 3.41 & $2 \cdot 23$ & 6.92 & CN8S59-2 \\
\hline 6.97 & 2.26 & 3.44 & $1 \cdot 39$ & 5.51 & 9.26 & 0.84 & 8.52 & 1.94 & 4.85 & DNES59-2 \\
\hline 6.18 & 9.17 & 1.99 & 7.77 & 4.00 & 10.77 & 4.78 & 3.01 & 6.99 & 9.99 & ENBS59-2 \\
\hline 2.50 & 1.94 & 0.74 & 4.25 & 10.56 & 5.29 & 3.67 & 7.45 & $1 \cdot 30$ & 6.14 & ANBS60-1 \\
\hline 6.50 & 1.52 & 7.91 & 3.97 & 5.61 & 10.93 & 4.59 & 0.72 & 2.27 & 2.80 & DNES60-1 \\
\hline 4.71 & 1.72 & 8.24 & 0.76 & 8.89 & $5 \cdot 23$ & 1.24 & 3.29 & 2.08 & 6.74 & CNES60-1 \\
\hline 7.08 & $2 \cdot 37$ & 3.57 & 1.49 & 5.64 & $9 \cdot 38$ & 0.97 & 8.63 & 2.06 & 4.98 & DNES60-1 \\
\hline 6.19 & 9.17 & 1.99 & 7.77 & 4.00 & 10,77 & 4.78 & 2.99 & 6.98 & 9.98 & ENES60-1 \\
\hline
\end{tabular}

-The NBS values are provided by Arie w. Hartman, Fred W. Rosberry (retired), and Dennis A. Swyt, Center for Manufacturing Engineering, NBS. 


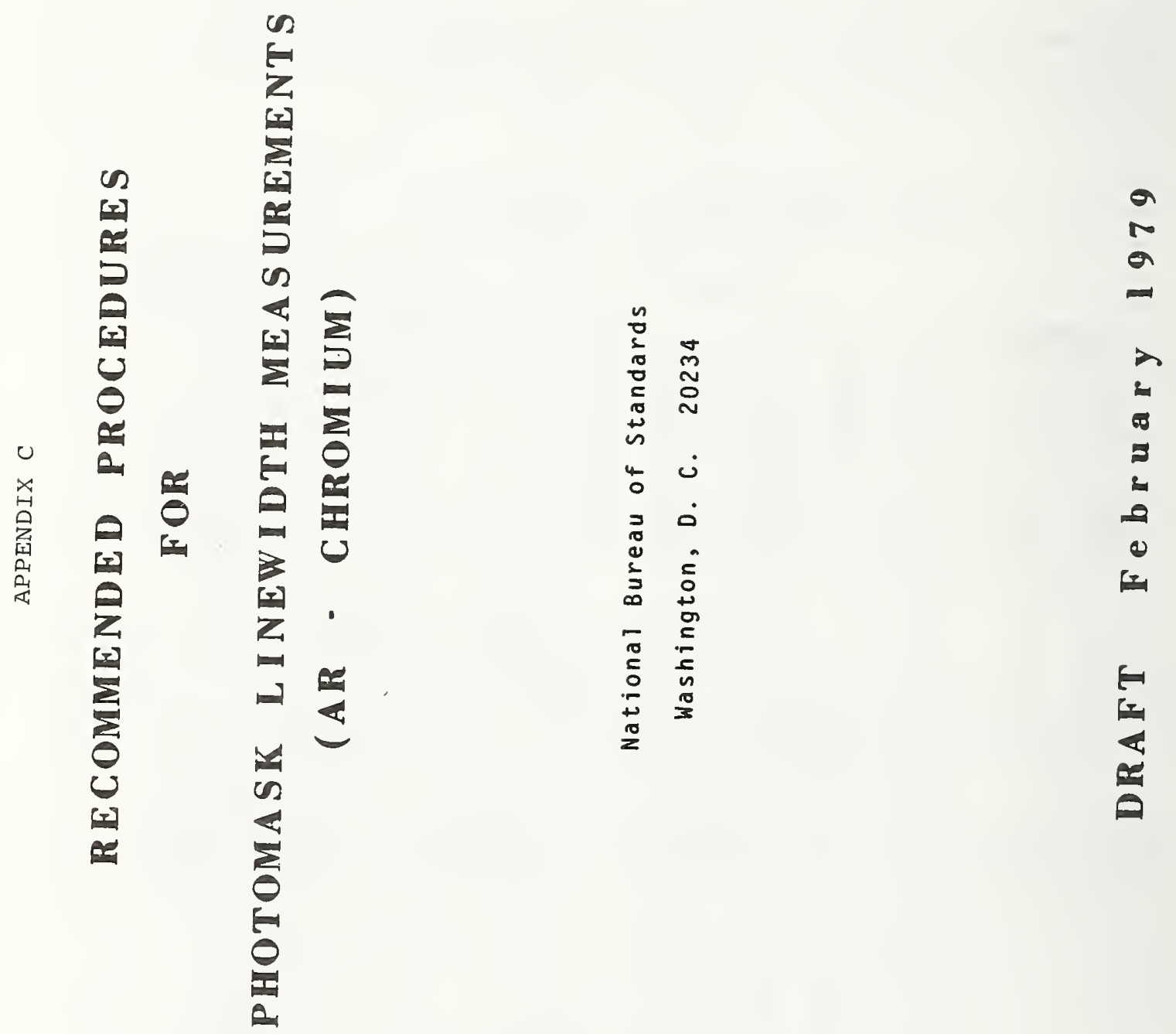




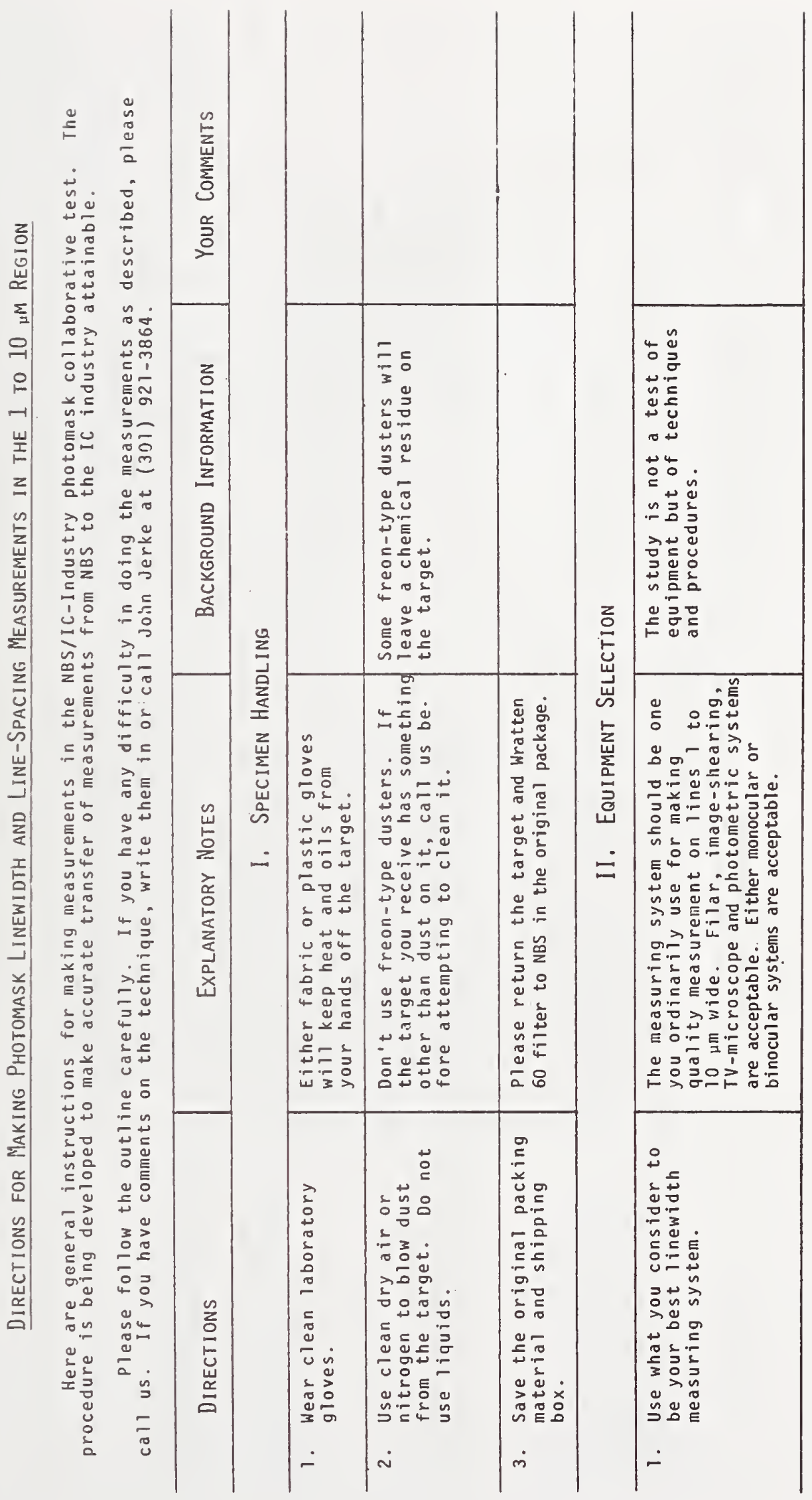




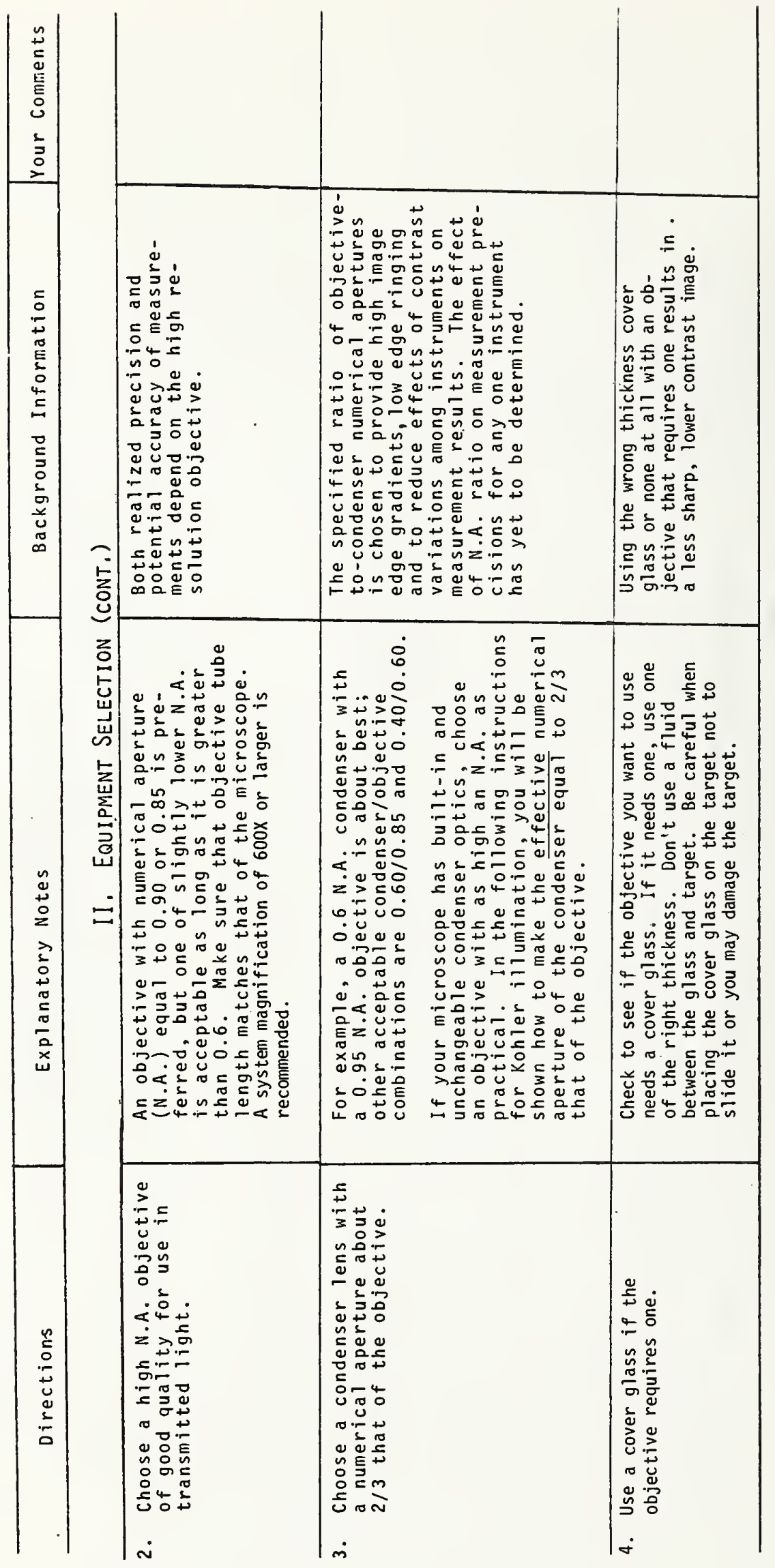




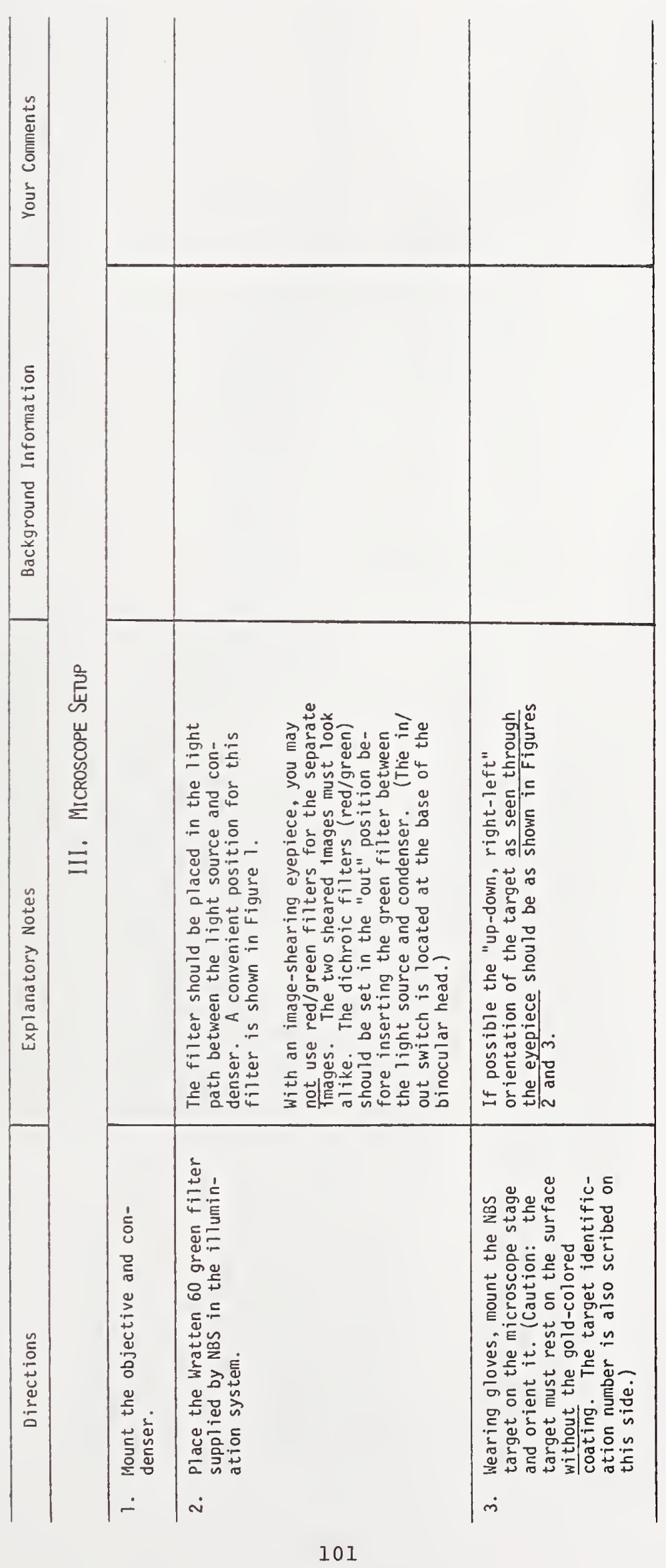




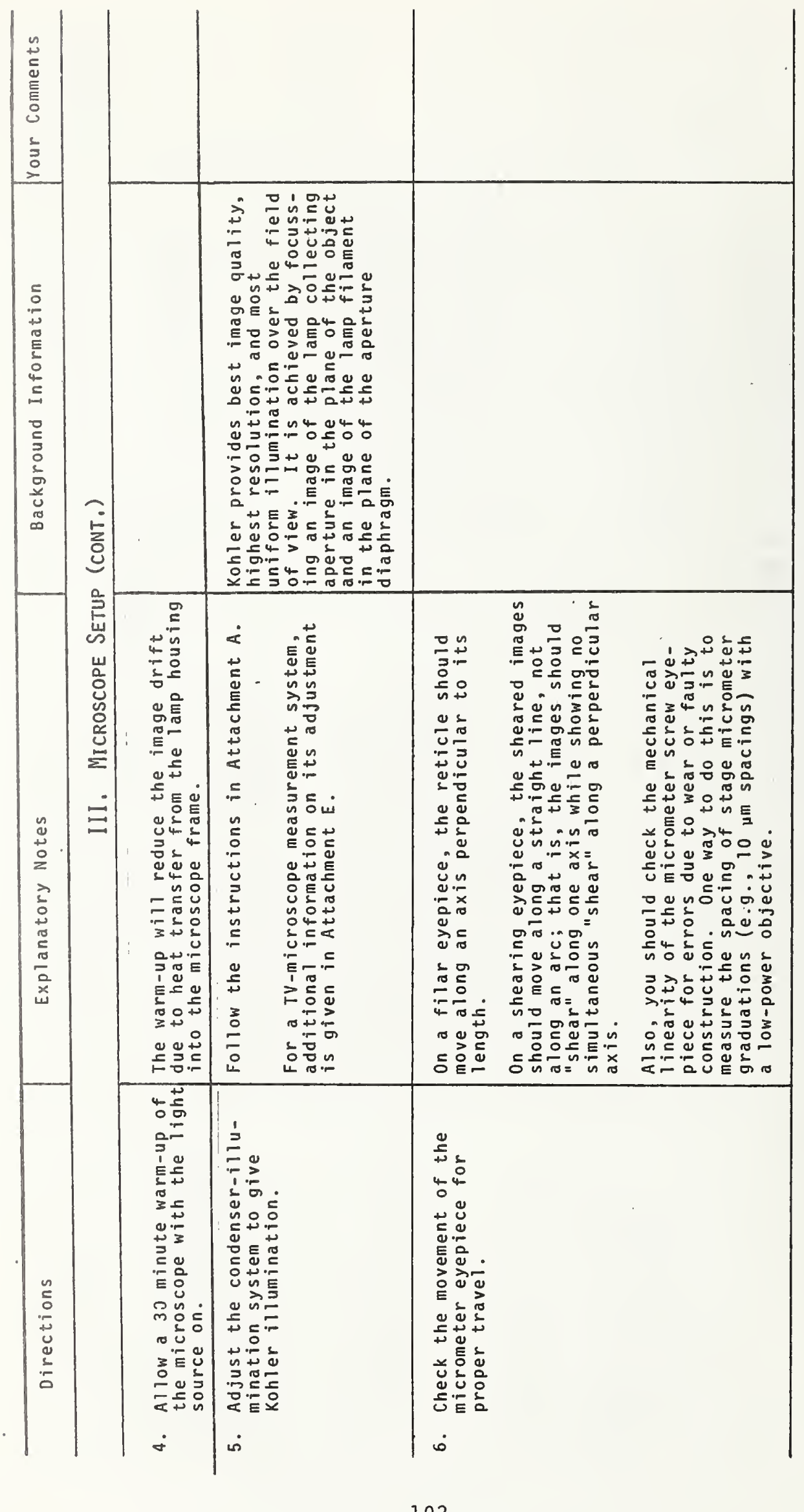




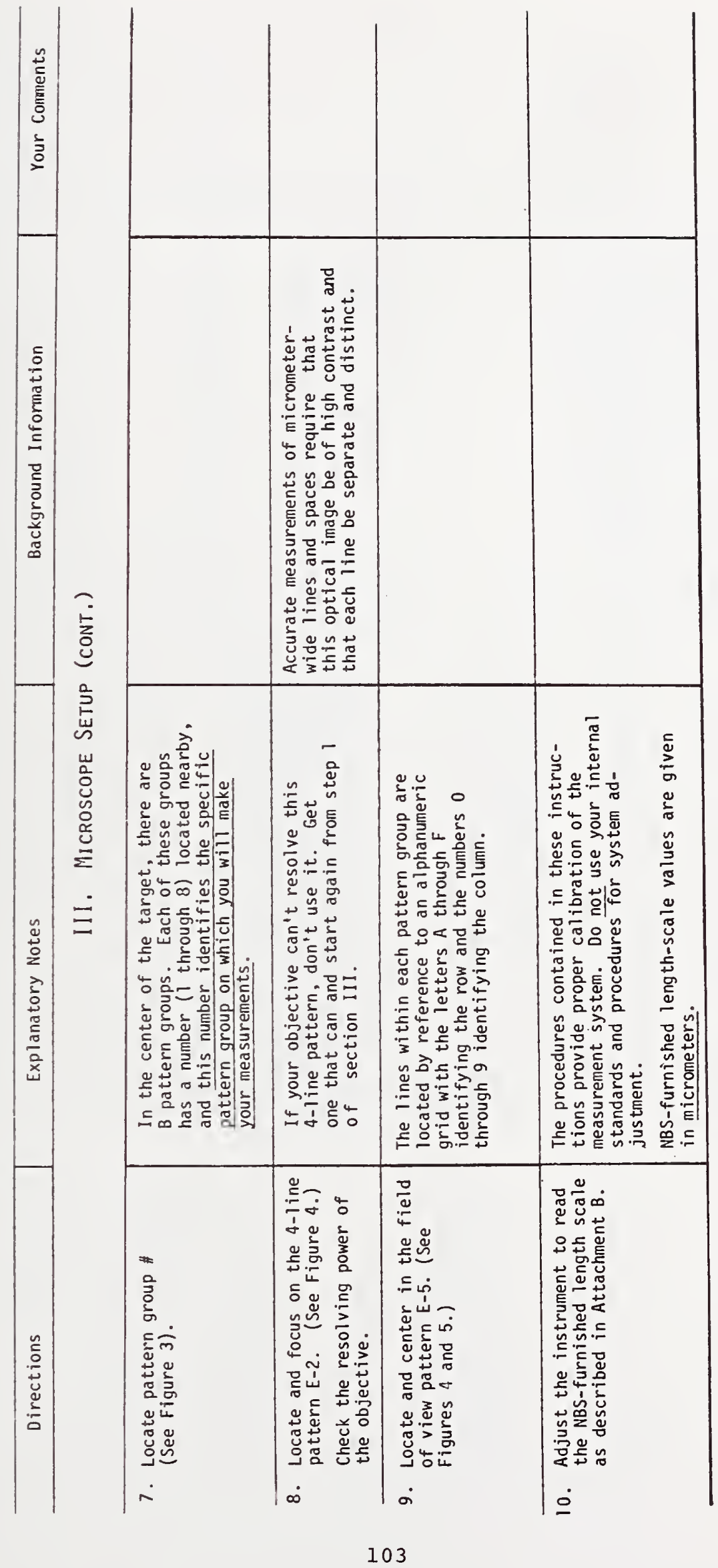




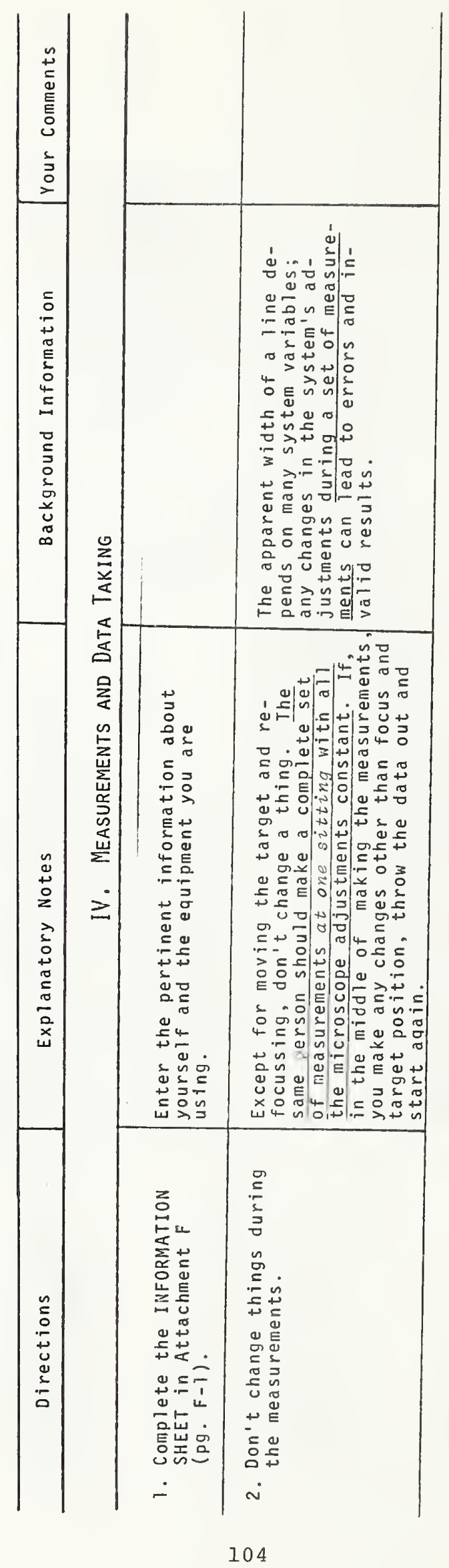




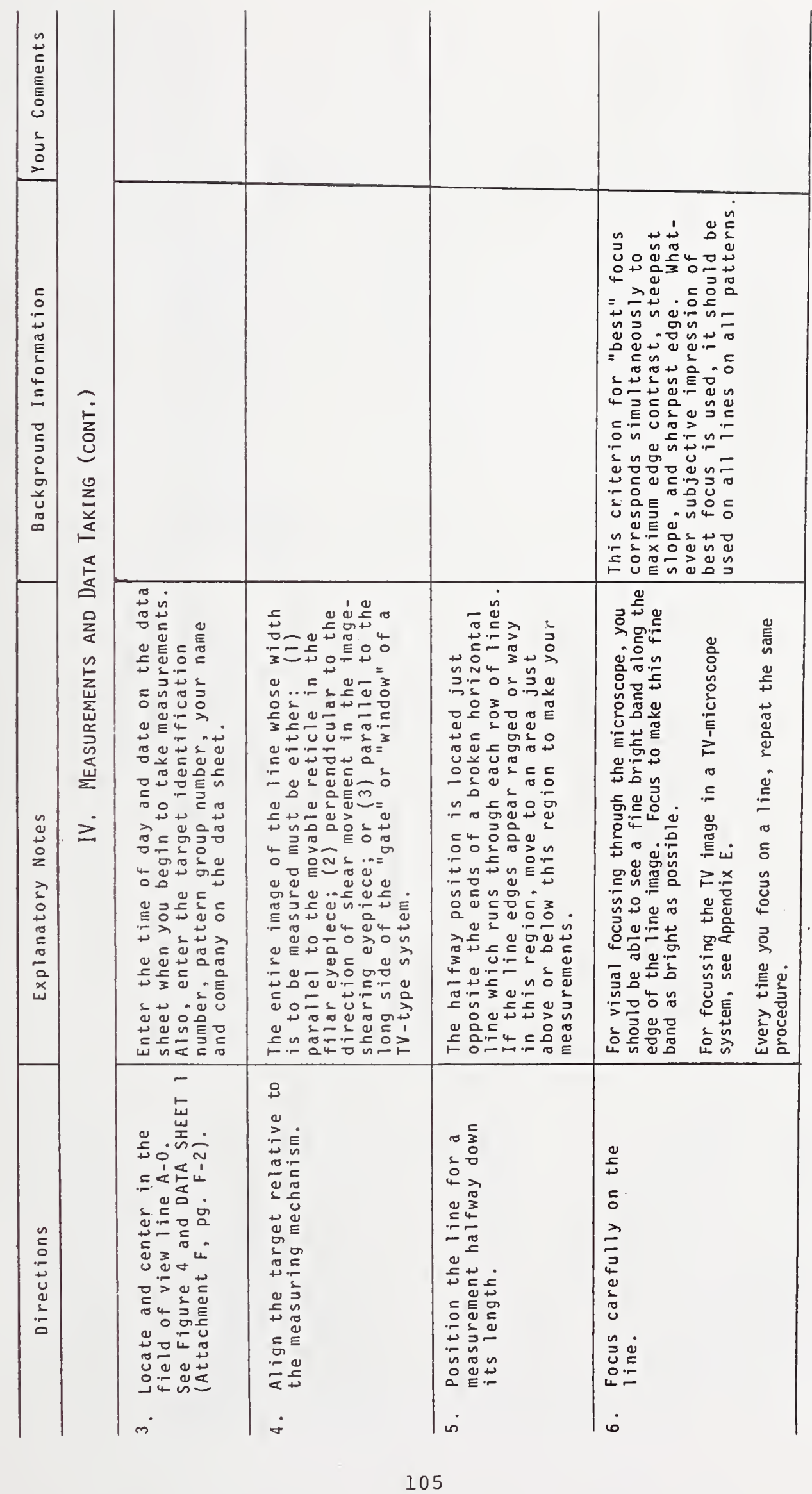




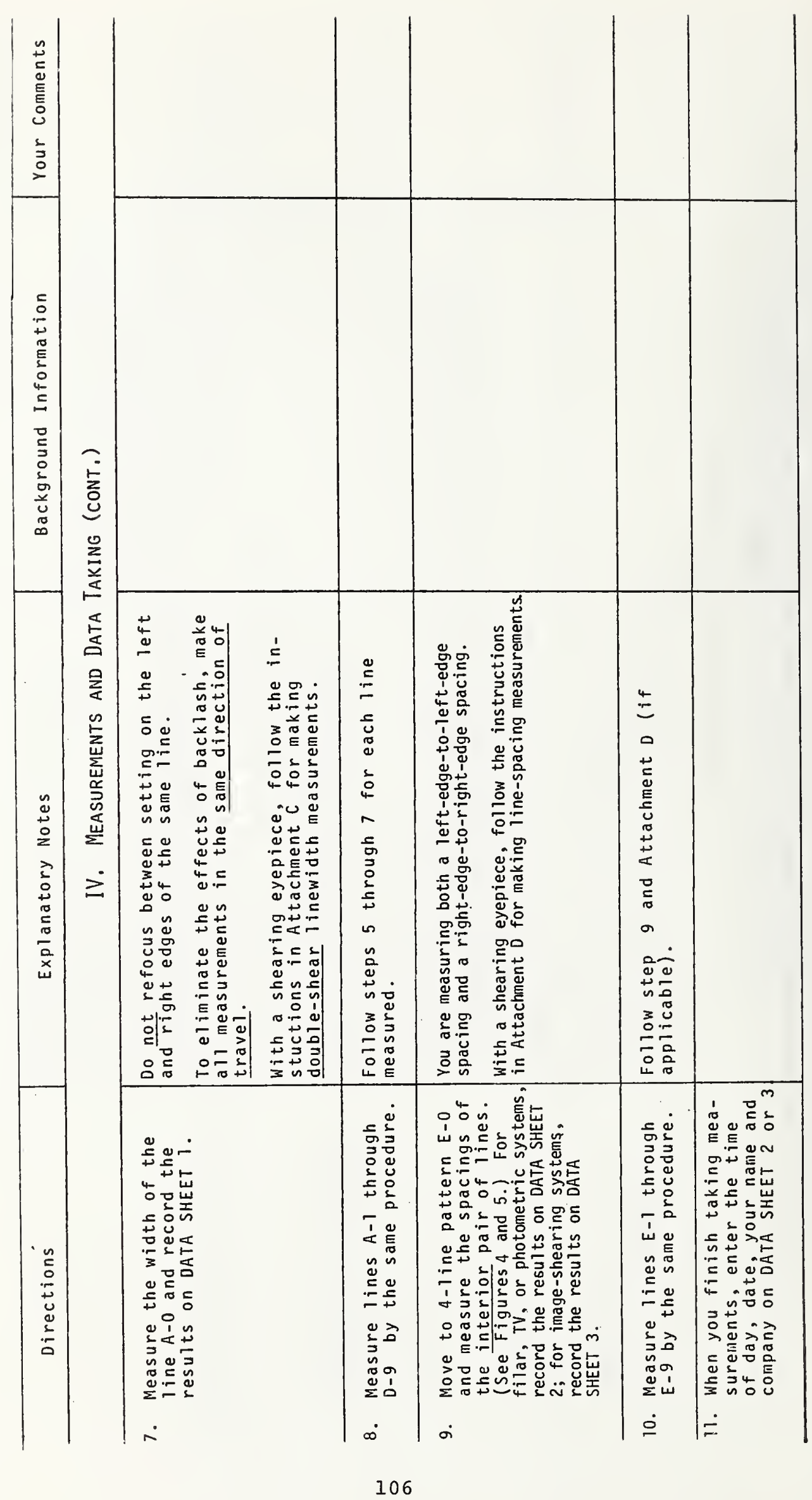




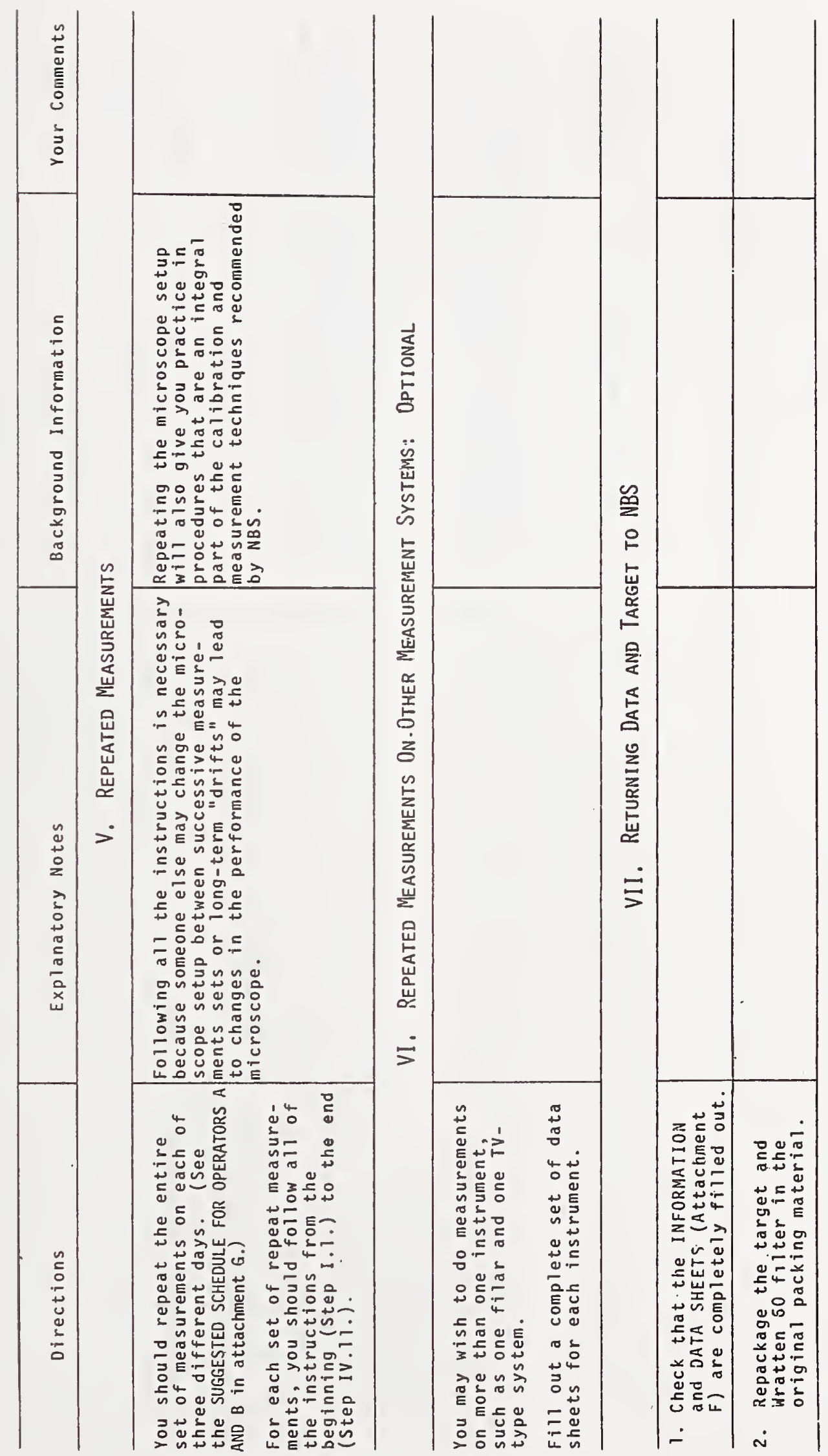




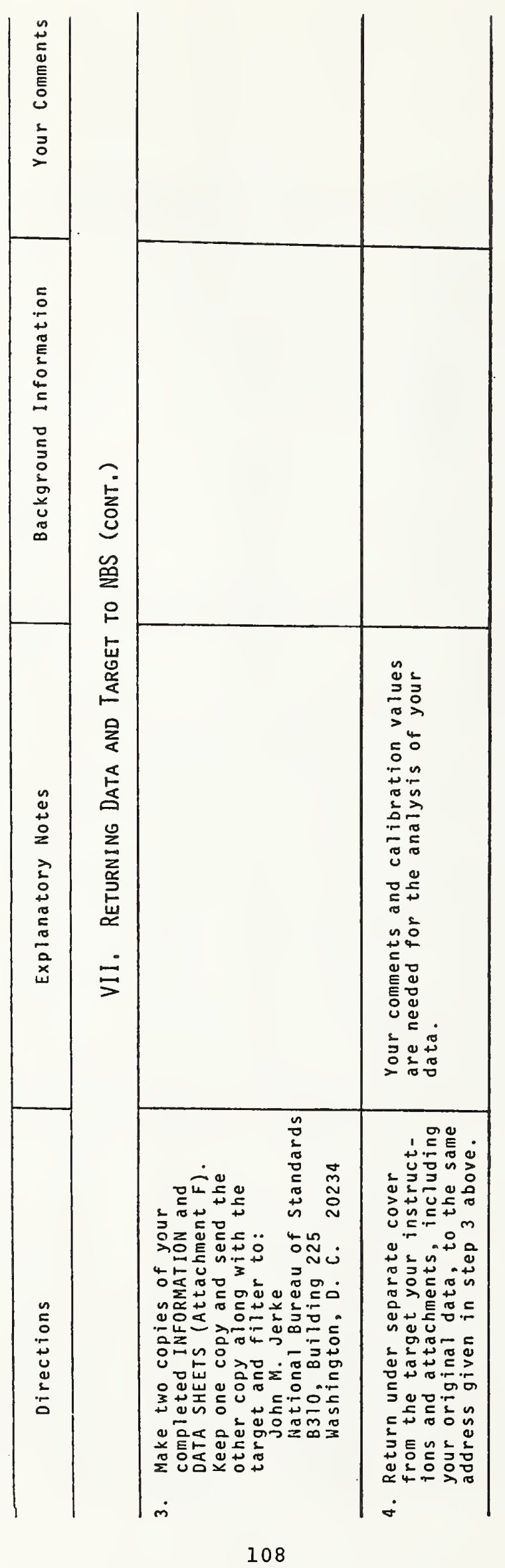




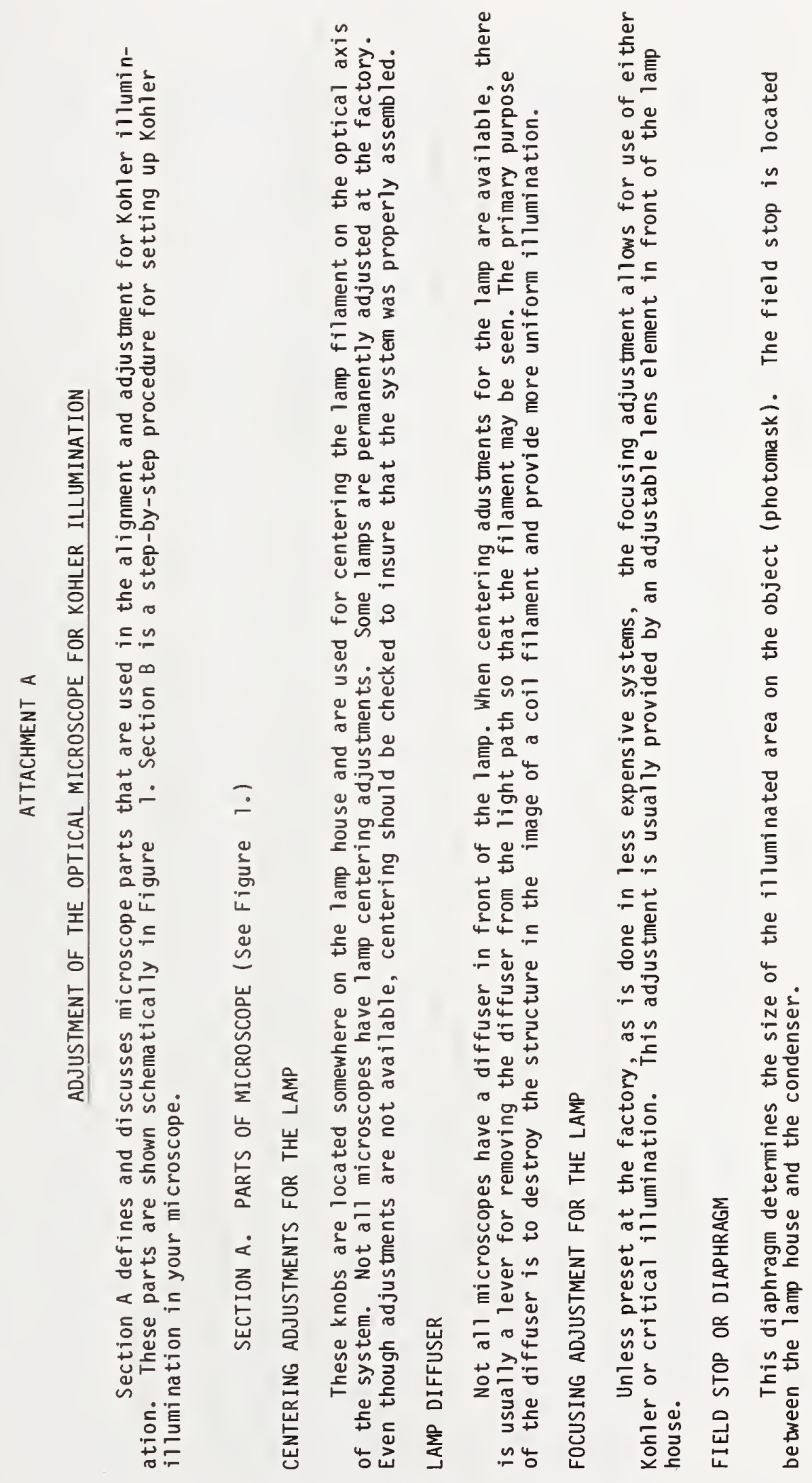




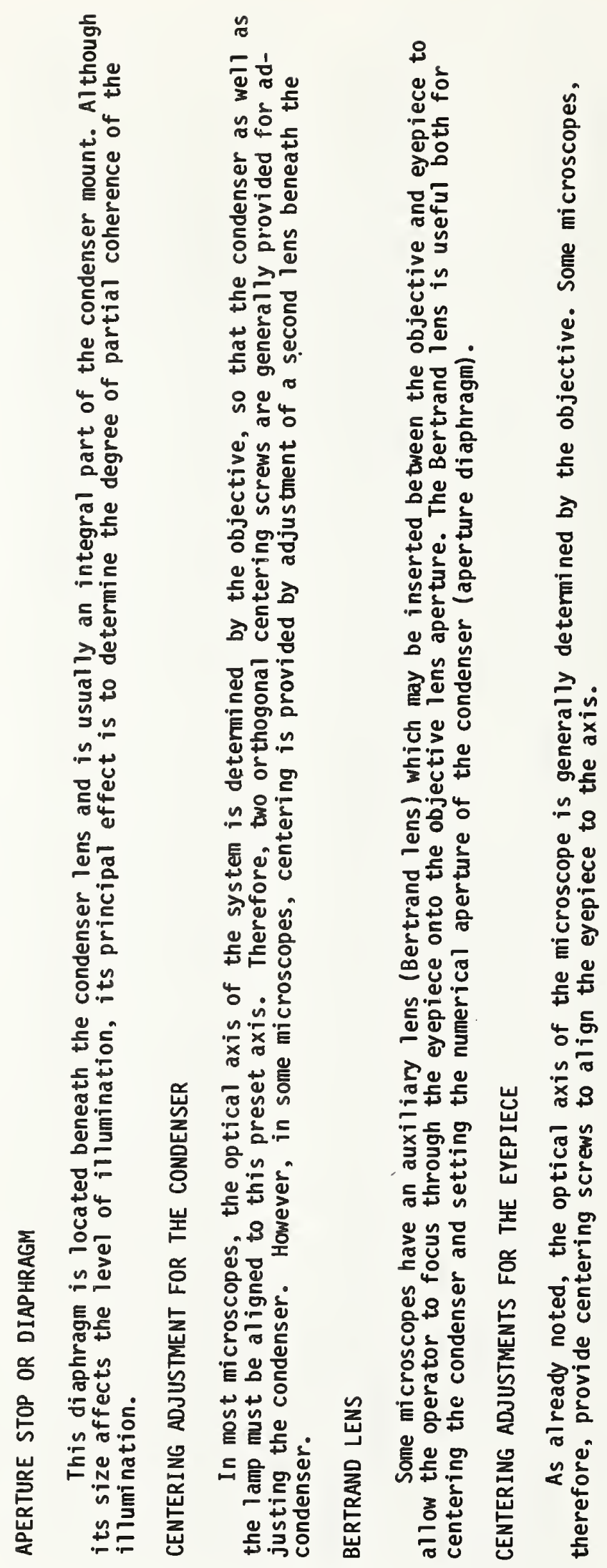




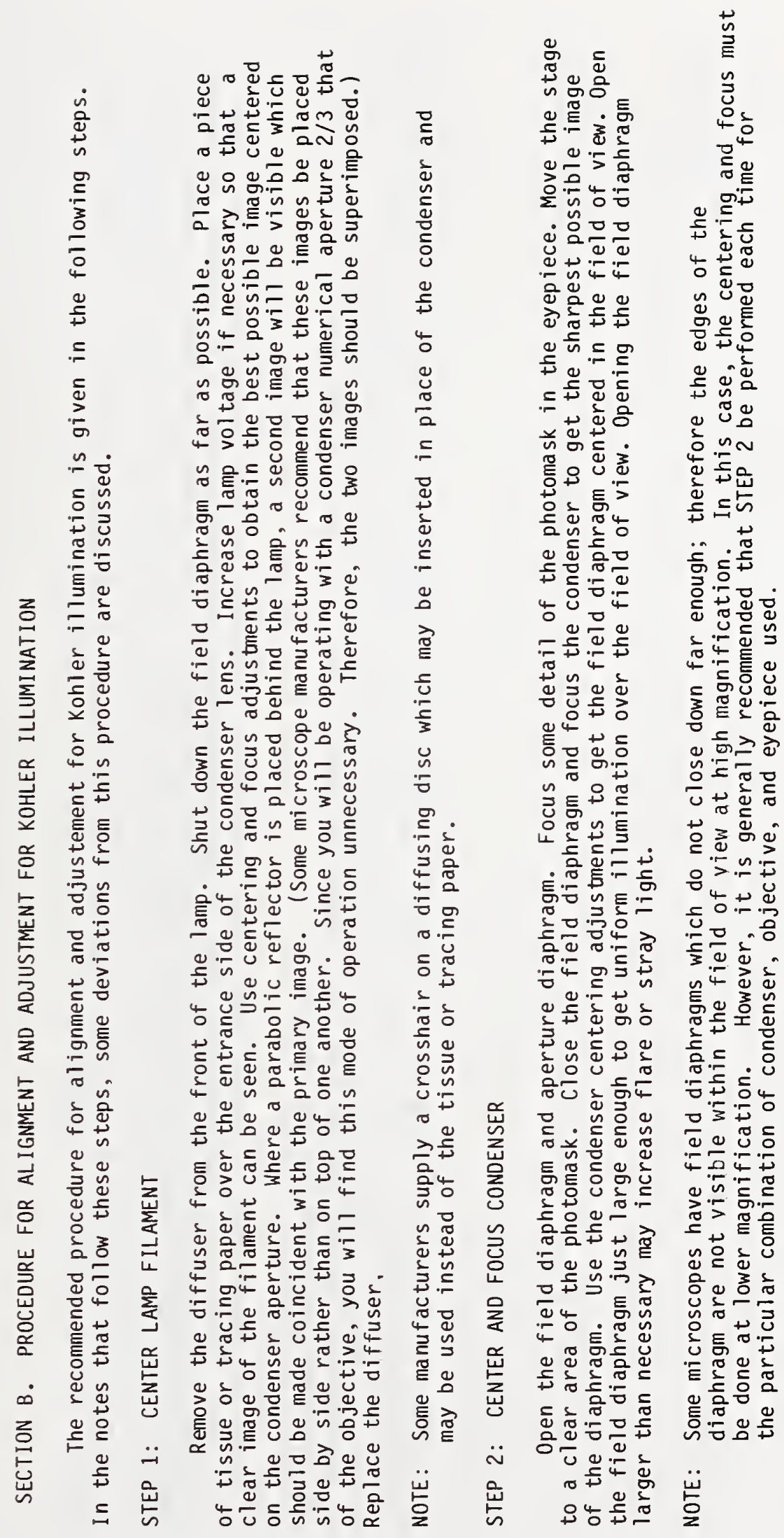




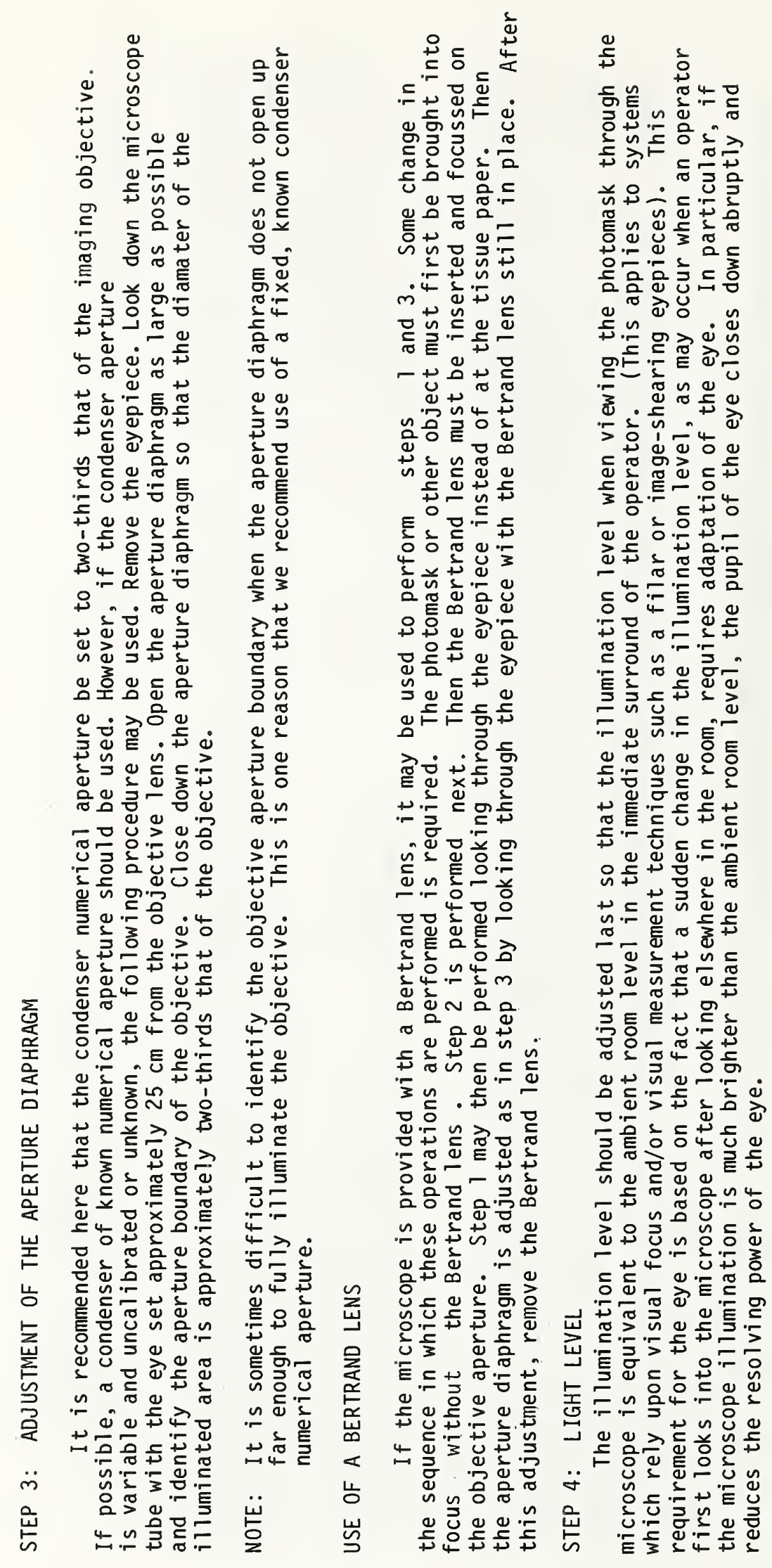




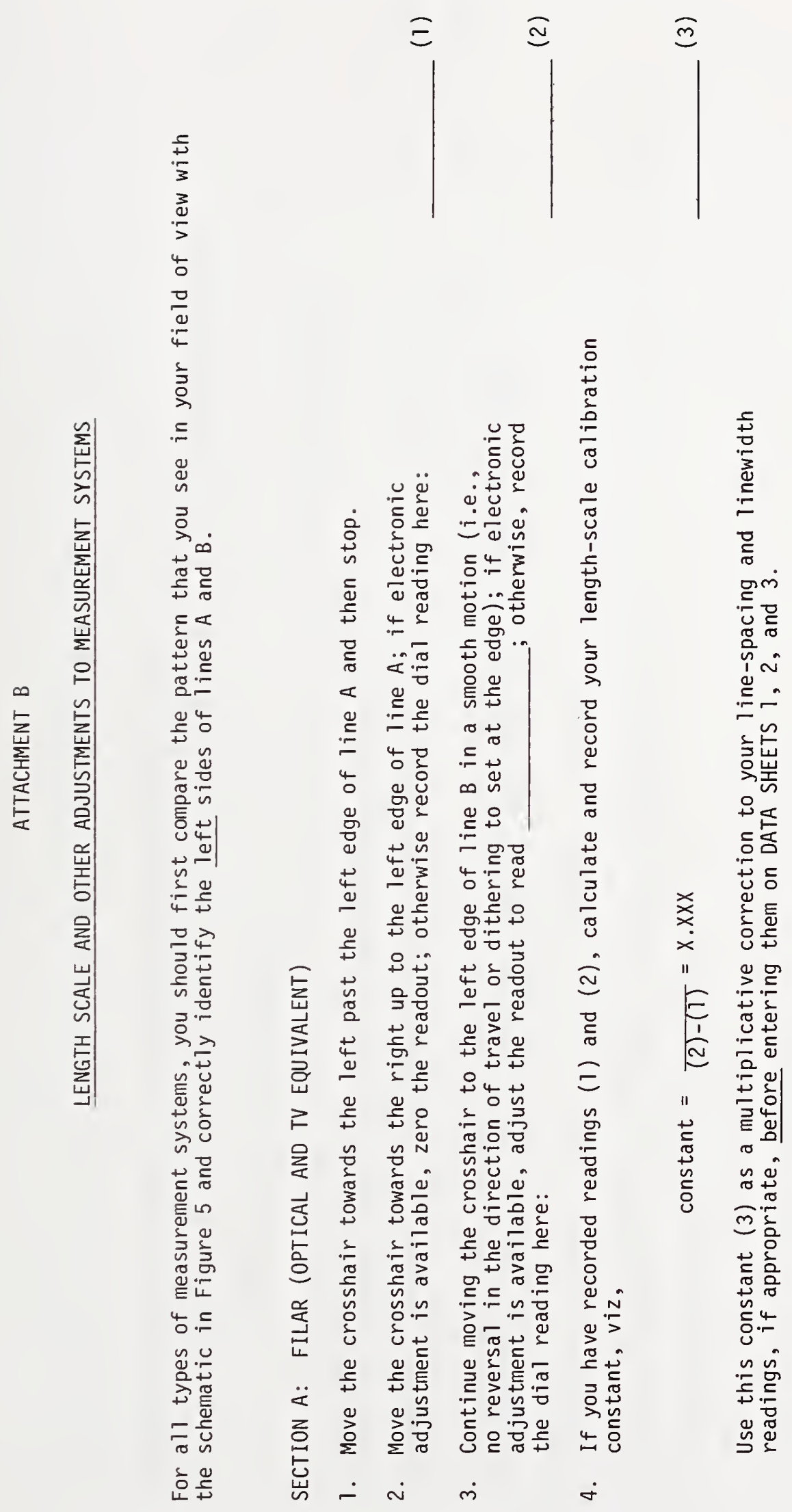



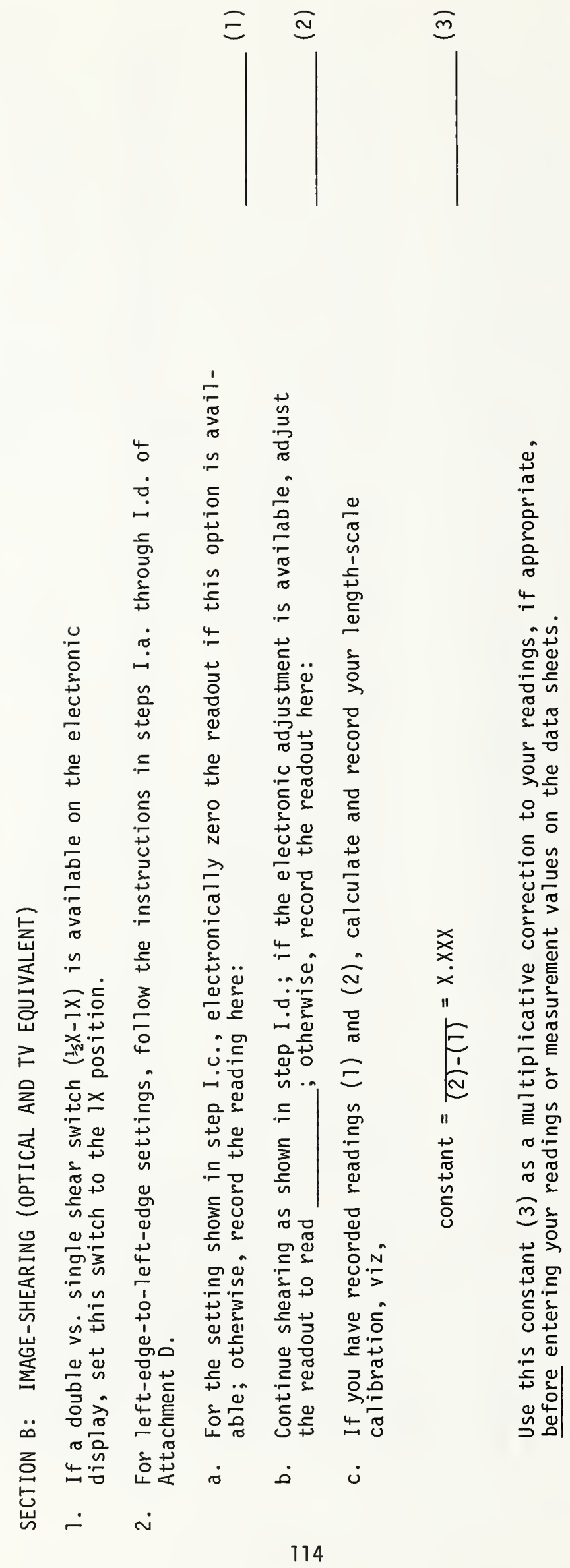


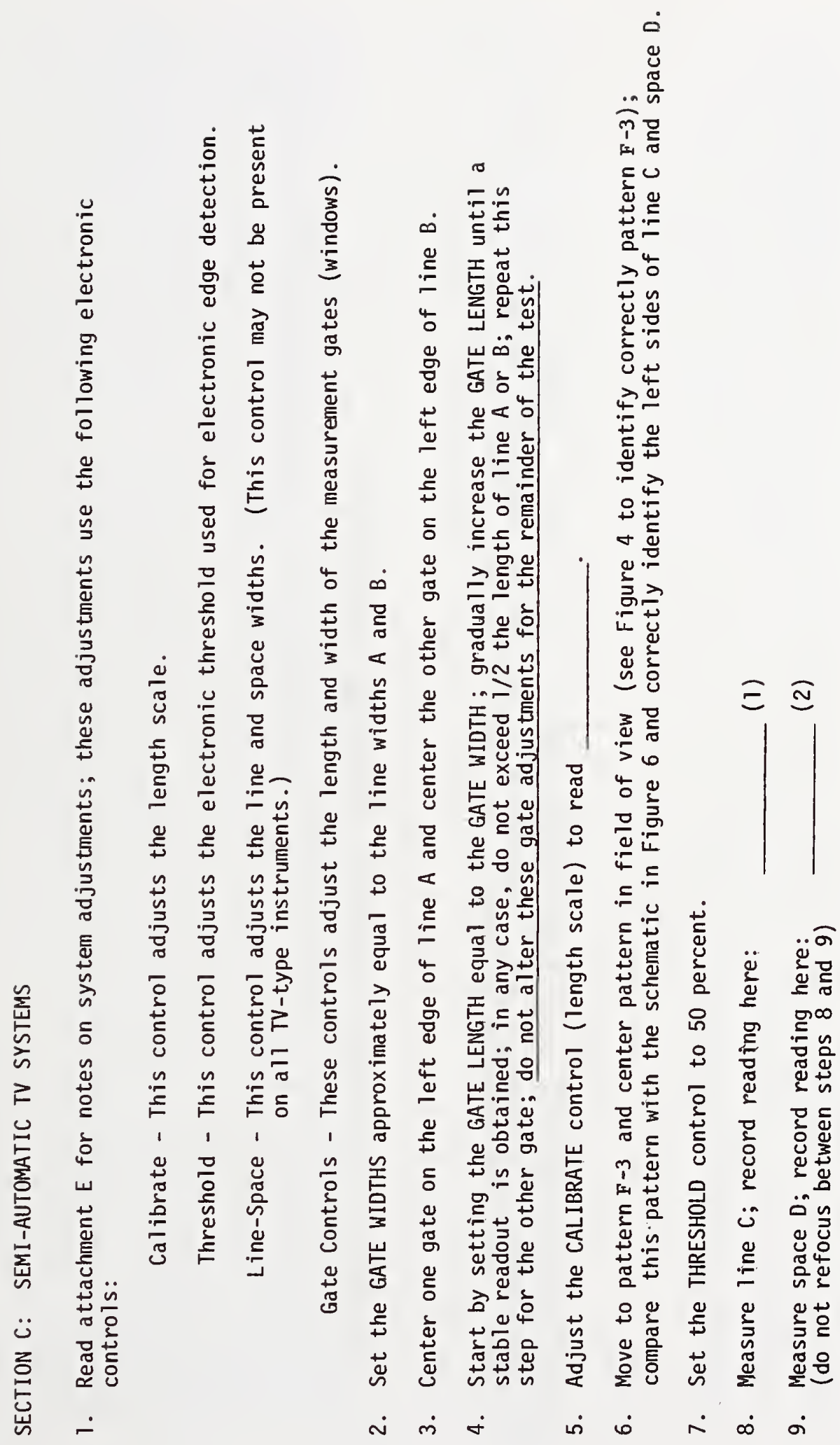


is 00

勇

त.

万。…으

岁创苛至

遇西

(

उิ) $=2$

4 过

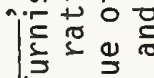

$4.2 \%$

雪

2

$\square=\frac{\pi}{5}$

$\Phi \subseteq 4$

00

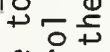

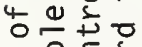

ข 등ㅇㅇㅇ

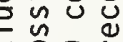

을옹

눈옹

饮出

대용

응

उ。

을 舟

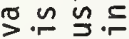

유.

동

ธ ธ

\& $\sim \frac{0}{\sigma}$

동동

可: 穴

은

든

주요ำ

등요원

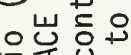

ॠ造

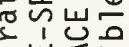

。岂高"

颌的

言崖㟔

恶嵒

$\dot{0}$ 


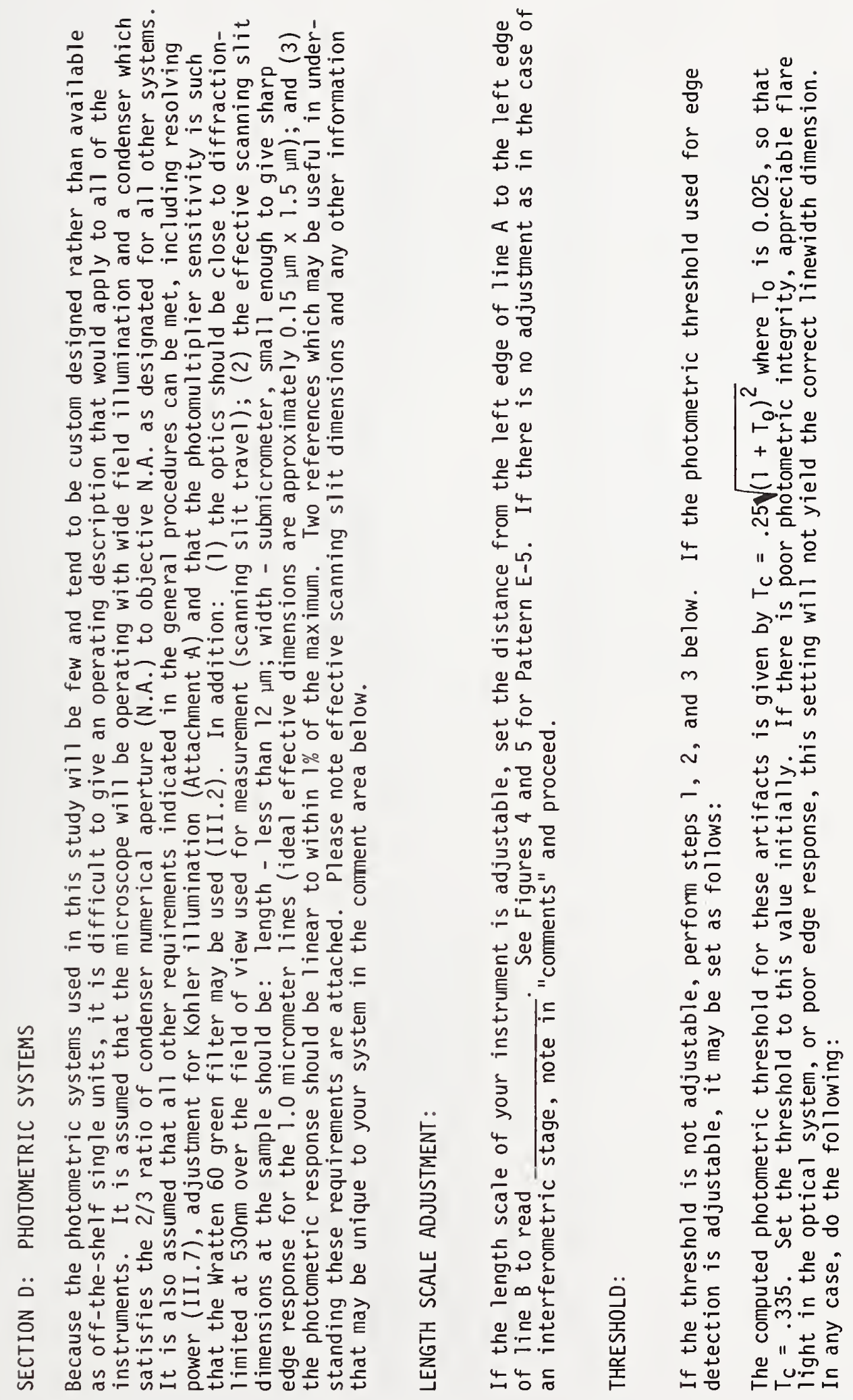




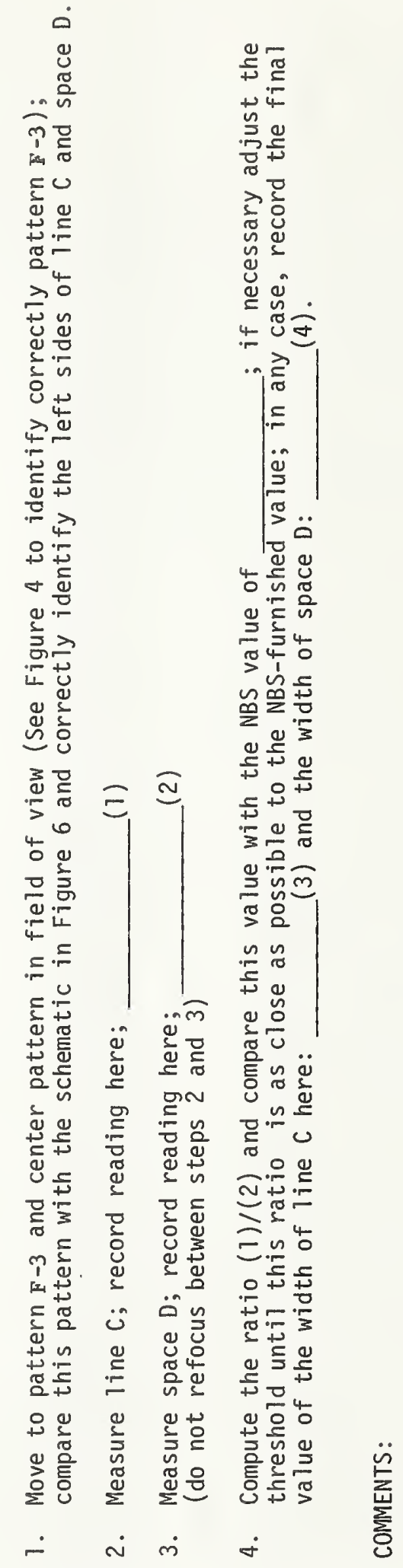




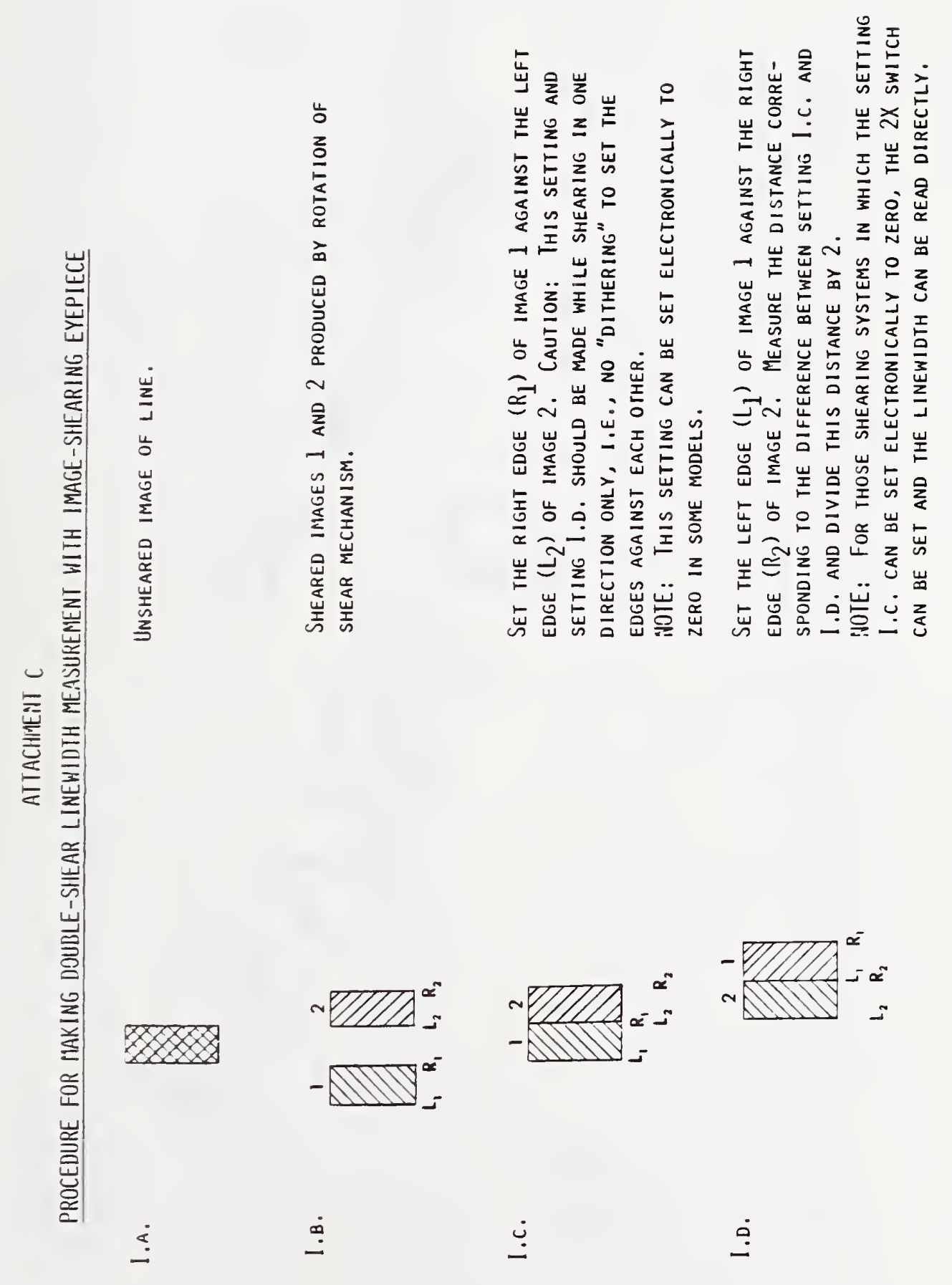




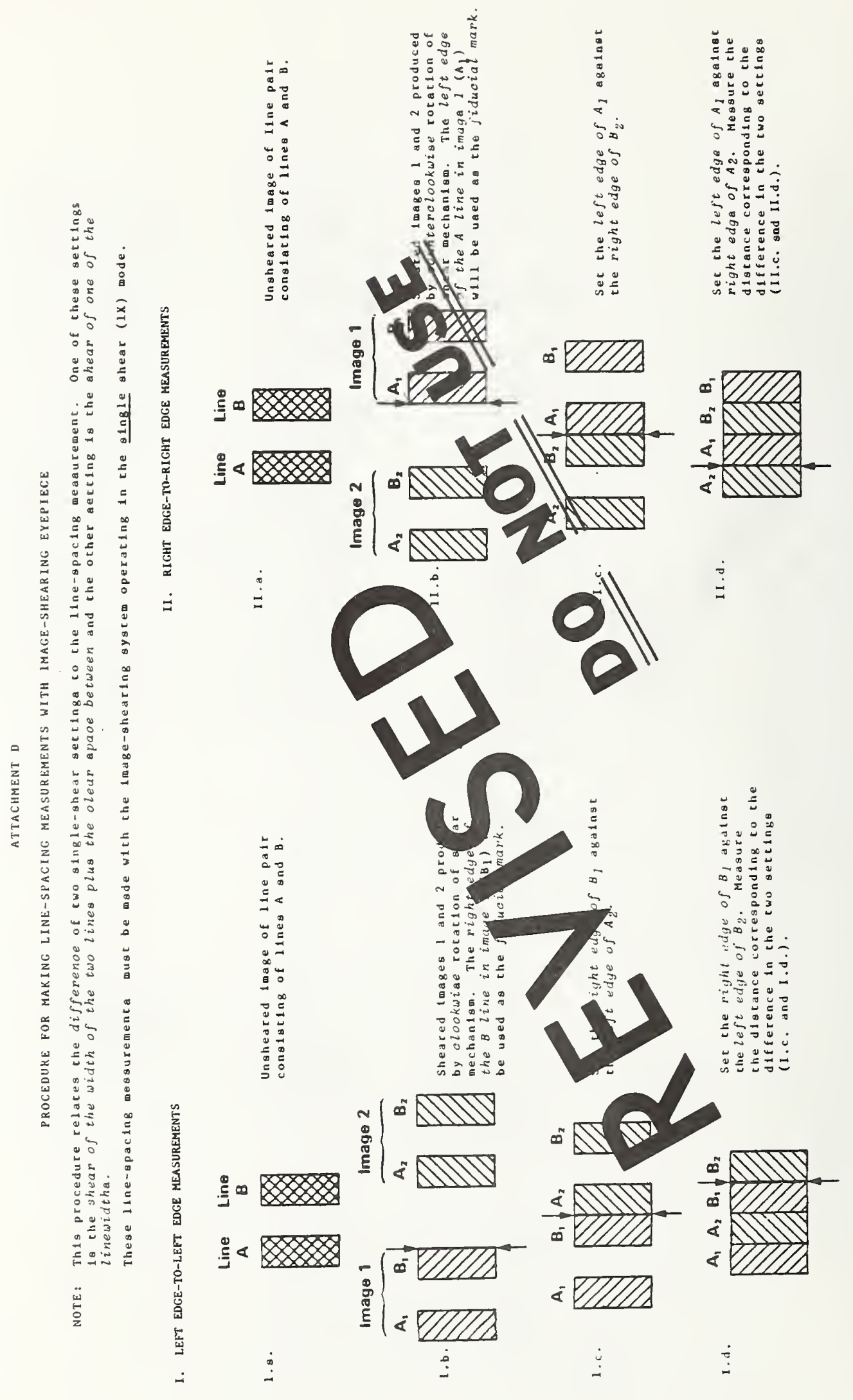


겅

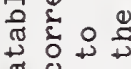

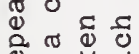

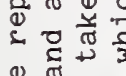

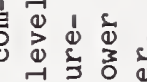

\% ธ

동용

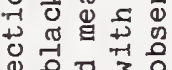

牙峲

응 उี 일 。․ำ

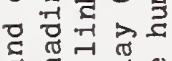

요 $\%$

व 0 क

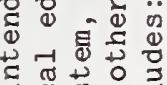

कू

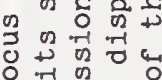

के के के

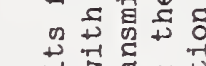

就

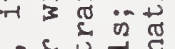

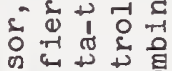

त这

ํㅣㄹ

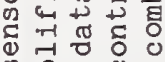

ป⿱一兀)

क

ธิ

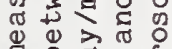

क क

क 2 ...

(5)

깅

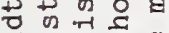

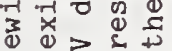

되

त् 2 स क

न है

प्र टूत

궁

c $\rightarrow$ व

언

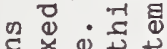

ธุ

पै हैं के

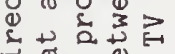

덤

(2)

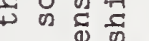

ญ

ऽ न

ช

ने है व

¿

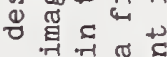

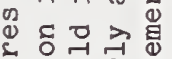

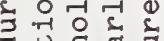

व

०

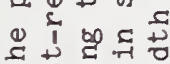

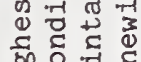

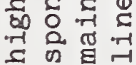

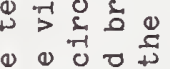

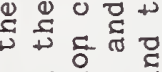

बं क्षे

ब

तै

ब开 0

के

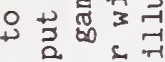

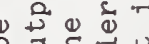

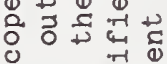

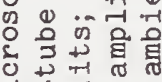

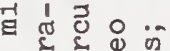

\& 0

of

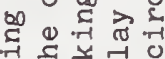

메

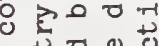

我密

ब.

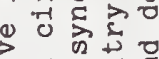

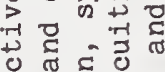

ब

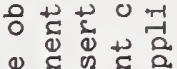

\&

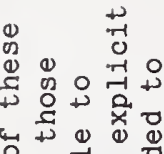

$\rightarrow$ 을

๙

स-

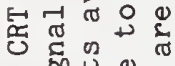

(1) 吕告

फ क

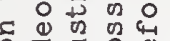

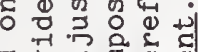

ช

$\rightarrow$ E 00

० त की क

ह ह

(2)

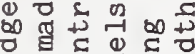

( ) 유유

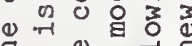

फ

ธ์ घ⿻

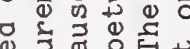

वे ठ

光

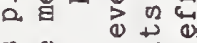

ने ฮ

๘

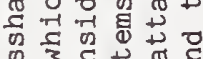

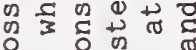

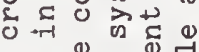

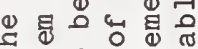

山े

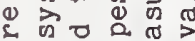

ब

$3 \varepsilon \approx \Sigma>\triangle$

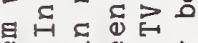

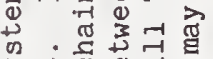

बे

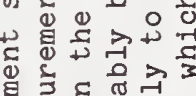

घट

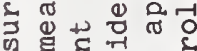

त्र

曰 ⿷匚

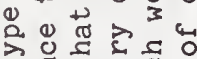

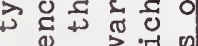

ส

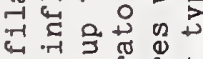

$>$ क ⿻上丨

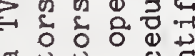

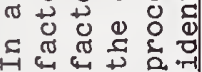

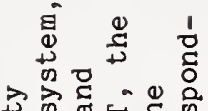

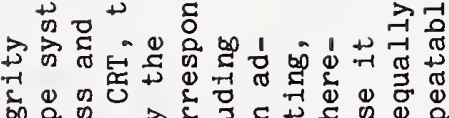

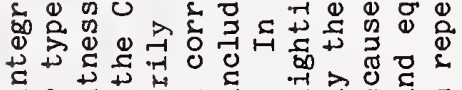

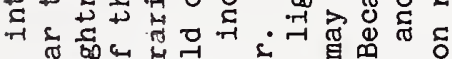

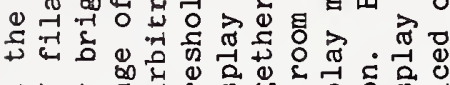

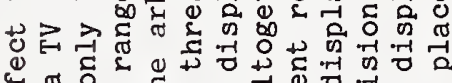

๘

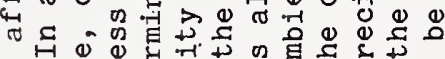

ร 0 D

至

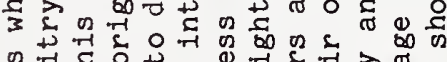
क ब $匚$ व

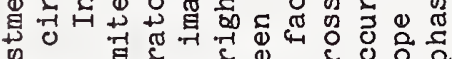

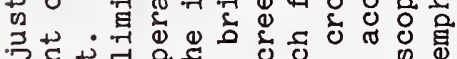
ठ임

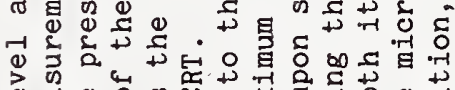

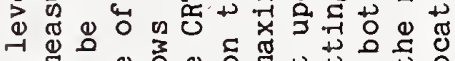

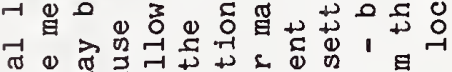

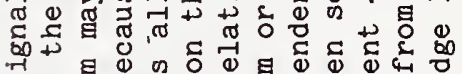
次寻㐫

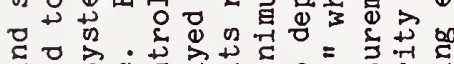

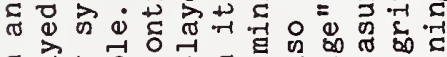
ᄃ

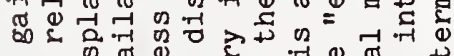
® न

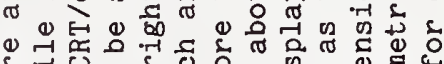

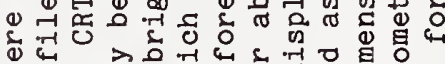

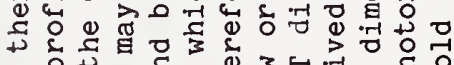

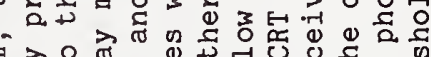

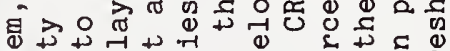

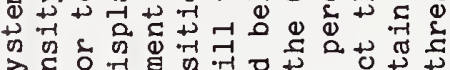
के

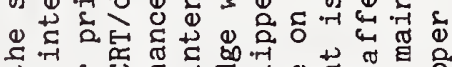

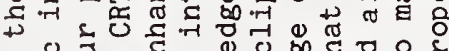
4 ص め)

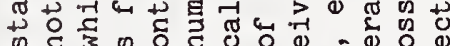

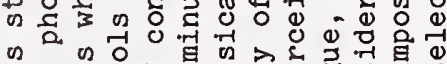

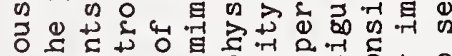

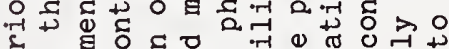

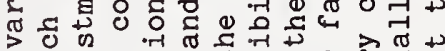

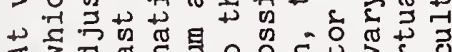

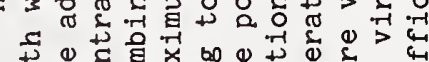

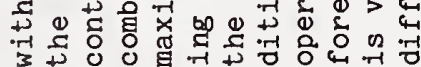




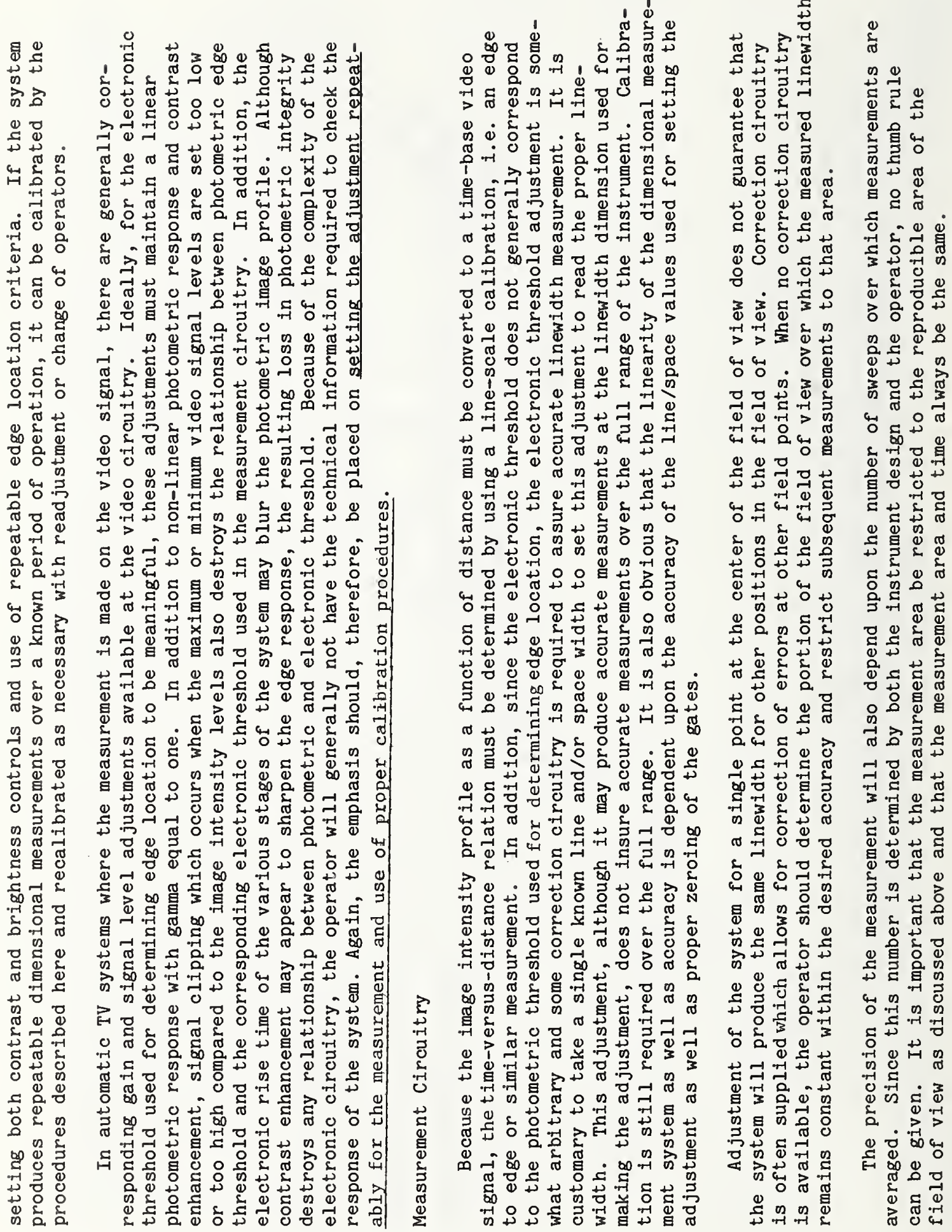




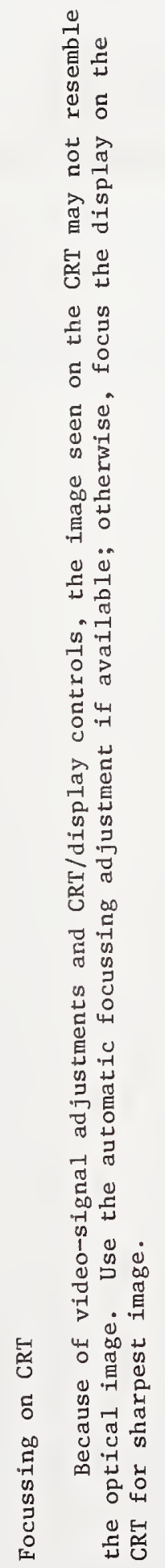


ATTACHMENT $F$

INFORMATION SHEET AND DATA SHEETS 
Company Name:

Company Mailing Address:

Company Phone Number:

Company Contact Name:

Phone Number:

Operators' Names:

Date:

NBS Target Number:

\section{Microscope Data}

Instrument Brand Name:

Model Name or Number:

Objective Lens:

Brand Name:

Type

Numerical Aperture

Cover Glass Required

No $\square$ Yes $\square$ Thickness: $\mathrm{mm}$

Tube Length: - $\mathrm{mm}$

Condenser Lens:

Brand Name

Type

Numerical Aperture

Built-in Condenser with Unknown N.A.

Measuring Device:

Type: $\quad$ Filar $\square \quad$ Image-shearing $\square$

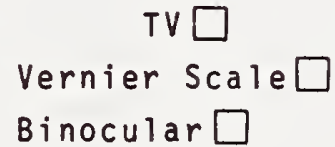

Read-out: Digital Display $\square$

Barrel Monocular $\square$

Binocular $\square$

Eyepiece Magnification $\square$

Overall Magnification Computation for Visual Systems:

Objective Lens Magnification:

$x$

Additional Magnification 1 :

$x$

Eyepiece (or viewing screen):

$x$

Overall Magnification

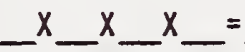


DATA SHEET 1

LINEWIDTH MEASUREMENTS

Target 非

Pattern Group \#

Date

Time

When you begin to take measurements

Your Name

Company

\begin{tabular}{|c|c|c|}
\hline Pattern & Type. & Measurement \\
\hline$A-0$ & Opaque LIne & \\
\hline$A=1$ & $" 1$ & \\
\hline$A-2$ & "I & \\
\hline$A-3$ & $"$ & \\
\hline$A=4$ & $" 1$ & \\
\hline$A-5$ & $"$ & \\
\hline$A-6$ & $"$ & \\
\hline$A-7$ & $"$ & \\
\hline$A-8$ & $"$ & \\
\hline$A-9$ & $"$ & \\
\hline $\mathrm{B}-\mathrm{O}$ & Clear Line & \\
\hline $\mathrm{B}-1$ & $"$ & \\
\hline$B-2$ & $"$ & \\
\hline$B-3$ & $" 1$ & \\
\hline$B-4$ & $" 1$ & \\
\hline $3-5$ & $"$ & \\
\hline $8-6$ & $" 1$ & - \\
\hline$B-7$ & $" 1$ & \\
\hline $\mathrm{B}-8$ & $"$ & \\
\hline$B-9$ & $"$ & \\
\hline$C-0$ & Opaque line & \\
\hline$C-1$ & $n$ & \\
\hline$C-2$ & $" 1$ & \\
\hline$C-3$ & $"$ & \\
\hline$C-4$ & $"$ & \\
\hline$C-5$ & $"$ & \\
\hline$c-6$ & $"$ & \\
\hline$C-7$ & $"$ & \\
\hline$C-8$ & $"$ & \\
\hline$C-9$ & $"$ & \\
\hline $\mathrm{D}-0$ & Clear Line & \\
\hline $\mathrm{D}-1$ & $"$ & \\
\hline $\mathrm{D}-2$ & $"$ & \\
\hline$D-3$ & $"$ & \\
\hline$D-4$ & $"$ & \\
\hline$D-5$ & $"$ & \\
\hline$D-6$ & $"$ & 1 \\
\hline$D-7$ & $"$ & \\
\hline$D-8$ & $"$ & \\
\hline$D-9$ & $" 1$ & \\
\hline
\end{tabular}


DATA SHEET 2

LINE-SPACING MEASUREMENTS

FILAR, TV, OR PHOTOMETRIC SYSTEM

\begin{tabular}{|c|c|c|}
\hline Pattern & Method & Measurement \\
\hline \multirow{2}{*}{$E-0$} & $\begin{array}{l}\text { Left edge of line A } \\
\text { to left edge of line B }\end{array}$ & \\
\hline & $\begin{array}{l}\text { Right edge of line A } \\
\text { to right edge of line B }\end{array}$ & \\
\hline \multirow{2}{*}{ E-1 } & $\begin{array}{l}\text { Left edge of line A } \\
\text { to left edge of line B }\end{array}$ & \\
\hline & $\begin{array}{l}\text { Right edge of line A } \\
\text { to right edge of line B }\end{array}$ & \\
\hline \multirow{2}{*}{$E-2$} & $\begin{array}{l}\text { Left edge of line A } \\
\text { to left edge of line B }\end{array}$ & \\
\hline & $\begin{array}{l}\text { Right edge of line A } \\
\text { to right edge of line B }\end{array}$ & \\
\hline \multirow{2}{*}{$E-3$} & $\begin{array}{l}\text { Left edge of line A } \\
\text { to left edge of line B }\end{array}$ & \\
\hline & $\begin{array}{l}\text { Right edge of line A } \\
\text { to right edge of line } B\end{array}$ & \\
\hline \multirow{2}{*}{ E-4 } & $\begin{array}{l}\text { Left edge of line A } \\
\text { to left edge of line B }\end{array}$ & \\
\hline & $\begin{array}{l}\text { Right edge of line A } \\
\text { to right edge of line B }\end{array}$ & \\
\hline \multirow{2}{*}{$E-5$} & $\begin{array}{l}\text { Left edge of line A } \\
\text { to left edge of line B }\end{array}$ & \\
\hline & $\begin{array}{l}\text { Right edge of line A } \\
\text { to right edge of line B }\end{array}$ & \\
\hline \multirow{2}{*}{ E-6 } & $\begin{array}{l}\text { Left edge of line A } \\
\text { to left edge of line } B\end{array}$ & \\
\hline & $\begin{array}{l}\text { Right edge of line A } \\
\text { to right edge of line } B\end{array}$ & \\
\hline \multirow{2}{*}{ E-7 } & $\begin{array}{l}\text { Left edge of line A } \\
\text { to left edge of line B }\end{array}$ & \\
\hline & $\begin{array}{l}\text { Right edge of line A } \\
\text { to right edge of line B }\end{array}$ & \\
\hline \multirow{2}{*}{ E-8 } & $\begin{array}{l}\text { Left edge of line A } \\
\text { to left edge of line B }\end{array}$ & \\
\hline & $\begin{array}{l}\text { Right edge of line A } \\
\text { to right edge of line B }\end{array}$ & \\
\hline \multirow{2}{*}{ E-9 } & $\begin{array}{l}\text { Left edge of line A } \\
\text { to left edge of line B }\end{array}$ & \\
\hline & $\begin{array}{l}\text { Right edge of line A } \\
\text { to right edge of line B }\end{array}$ & \\
\hline
\end{tabular}


DATA SHEET 3

LINE-SPACING MEASUREMENTS

IMAGE-SHEARING SYSTEM

NOTE: USE ONLY THE SHEAR TECHNIQUE AS DESCRIBED IN ATTACHMENT D.

\begin{tabular}{|c|c|c|c|}
\hline Pattern & Method & Reading & $\begin{array}{c}\text { Measurement } \\
\text { (Difference in } \\
\text { Readings) }\end{array}$ \\
\hline \multirow{4}{*}{$E-0$} & $\begin{array}{l}\text { Right edge of } B_{1} \\
\text { against left edge of } A_{2}\end{array}$ & & \\
\hline & $\begin{array}{l}\text { Right edge of } B_{1} \\
\text { agalnst left edge of } B_{2}\end{array}$ & & \\
\hline & $\begin{array}{l}\text { Left edge of } A_{1} \\
\text { against right edge of } B_{2}\end{array}$ & & \\
\hline & $\begin{array}{l}\text { Left edge of } A_{1} \\
\text { against right edge of } A_{2}\end{array}$ & & \\
\hline \multirow{4}{*}{ E-1 } & $\begin{array}{l}\text { Right edge of } B_{1} \\
\text { agalngt left edge of } A_{2}\end{array}$ & & \\
\hline & $\begin{array}{l}\text { Right edge of } B_{1} \\
\text { agalnst left edge of } B_{2}\end{array}$ & & \\
\hline & $\begin{array}{l}\text { Left edge of } A_{1} \\
\text { againgt right edge of } B_{2}\end{array}$ & & \\
\hline & $\begin{array}{l}\text { Left edge of } A_{1} \\
\text { against right edge of } A_{2}\end{array}$ & & \\
\hline \multirow{4}{*}{ E-2 } & $\begin{array}{l}\text { Right edge of } B_{1} \\
\text { agalnst left edge of } A_{2}\end{array}$ & & \\
\hline & $\begin{array}{l}\text { Right edge of } B_{1} \\
\text { against left edge of } B_{2}\end{array}$ & " & \\
\hline & $\begin{array}{l}\text { Left edge of } A_{1} \\
\text { against right edge of } B_{2}\end{array}$ & & \\
\hline & $\begin{array}{l}\text { Left edge of } A_{1} \\
\text { againgt right edge of } A_{2}\end{array}$ & & \\
\hline \multirow{4}{*}{$E-3$} & $\begin{array}{l}\text { Right edge of } B_{1} \\
\text { against left edge of } A_{2}\end{array}$ & & \\
\hline & $\begin{array}{l}\text { Right edge of } \mathrm{B}_{1} \\
\text { against left edge of } \mathrm{B}_{2}\end{array}$ & & \\
\hline & $\begin{array}{l}\text { Left edge of } A_{1} \\
\text { against right edge of } B_{2}\end{array}$ & & \\
\hline & $\begin{array}{l}\text { Left edge of } A_{1} \\
\text { against right edge of } A_{2}\end{array}$ & & \\
\hline \multirow{4}{*}{ E-4 } & $\begin{array}{l}\text { Right edge of } B_{1} \\
\text { againgt left edge of } A_{2}\end{array}$ & & \\
\hline & $\begin{array}{l}\text { Right edge of } B_{1} \\
\text { against left edge of } B_{2}\end{array}$ & & \\
\hline & $\begin{array}{l}\text { Left edge of } A_{1} \\
\text { againgt right edge of } B_{2}\end{array}$ & & \\
\hline & $\begin{array}{l}\text { Left edge of } A_{1} \\
\text { against right edge of } A_{2}\end{array}$ & & \\
\hline
\end{tabular}


DATA SHEET 3 (concluded)

\begin{tabular}{|c|c|c|c|}
\hline Pattern & Method & Read1ng & $\begin{array}{l}\text { Measurement } \\
\text { (D1fference in } \\
\text { Readings) }\end{array}$ \\
\hline \multirow{4}{*}{$E-5$} & $\begin{array}{l}\text { Right edge of } B_{1} \\
\text { against left edge of } A_{2}\end{array}$ & & \multirow[t]{2}{*}{ ) } \\
\hline & $\begin{array}{l}\text { Right edge of } B_{1} \\
\text { against left edge of } B_{2}\end{array}$ & & \\
\hline & $\begin{array}{l}\text { Left edge of } A_{1} \\
\text { against right edge of } B_{2}\end{array}$ & & \\
\hline & $\begin{array}{l}\text { Left edge of } A_{1} \\
\text { against right edge of } A_{2}\end{array}$ & & \\
\hline \multirow{4}{*}{ E-6 } & $\begin{array}{l}\text { Right edge of } B_{1} \\
\text { against left edge of } A_{2}\end{array}$ & & \multirow{2}{*}{ 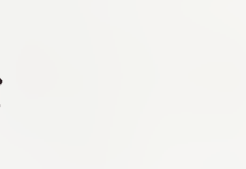 } \\
\hline & $\begin{array}{l}\text { R1ght edge of } B_{1} \\
\text { against left edge of } B_{2}\end{array}$ & & \\
\hline & $\begin{array}{l}\text { Left edge of } A_{1} \\
\text { against right edge of } B_{2}\end{array}$ & & \\
\hline & $\begin{array}{l}\text { Left edge of } A_{1} \\
\text { against right edge of } A_{2}\end{array}$ & & \\
\hline \multirow{4}{*}{$E-7$} & $\begin{array}{l}\text { Right edge of } B_{1} \\
\text { against left edge of } A_{2}\end{array}$ & & \\
\hline & $\begin{array}{l}\text { Right edge of } B_{1} \\
\text { against left edge of } B_{2}\end{array}$ & & \\
\hline & $\begin{array}{l}\text { Left edge of } A_{1} \\
\text { against right edge of } B_{2}\end{array}$ & & \\
\hline & $\begin{array}{l}\text { Left edge of } A_{1} \\
\text { against right edge of } A_{2}\end{array}$ & & \\
\hline \multirow{4}{*}{ E-8 } & $\begin{array}{l}\text { Right edge of } B_{1} \\
\text { agalnst left edge of } A_{2}\end{array}$ & & \\
\hline & $\begin{array}{l}\text { Right edge of } B_{1} \\
\text { against left edge of } B_{2}\end{array}$ & & \\
\hline & $\begin{array}{l}\text { Left edge of } A_{1} \\
\text { against right edge of } B_{2}\end{array}$ & & \multirow[b]{2}{*}{ 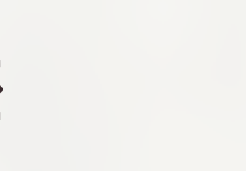 } \\
\hline & $\begin{array}{l}\text { Left edge of } A_{1} \\
\text { against right edge of } A_{2}\end{array}$ & & \\
\hline \multirow{4}{*}{$E-9$} & $\begin{array}{l}\text { Right edge of } B_{1} \\
\text { against left edge of } A_{2}\end{array}$ & & \\
\hline & $\begin{array}{l}\text { Right edge of } A_{1} \\
\text { against left edge of } B_{2}\end{array}$ & & \\
\hline & $\begin{array}{l}\text { Left edge of } A_{1} \\
\text { against right edge of } B_{2}\end{array}$ & & \\
\hline & $\begin{array}{l}\text { Left edge of } A_{1} \\
\text { against right edge of } A_{2}\end{array}$ & & \\
\hline
\end{tabular}

$\left.\begin{array}{l}\text { Date } \\ \text { Time }\end{array}\right\}$ when you finish taking measurements.

Your Name/Company 


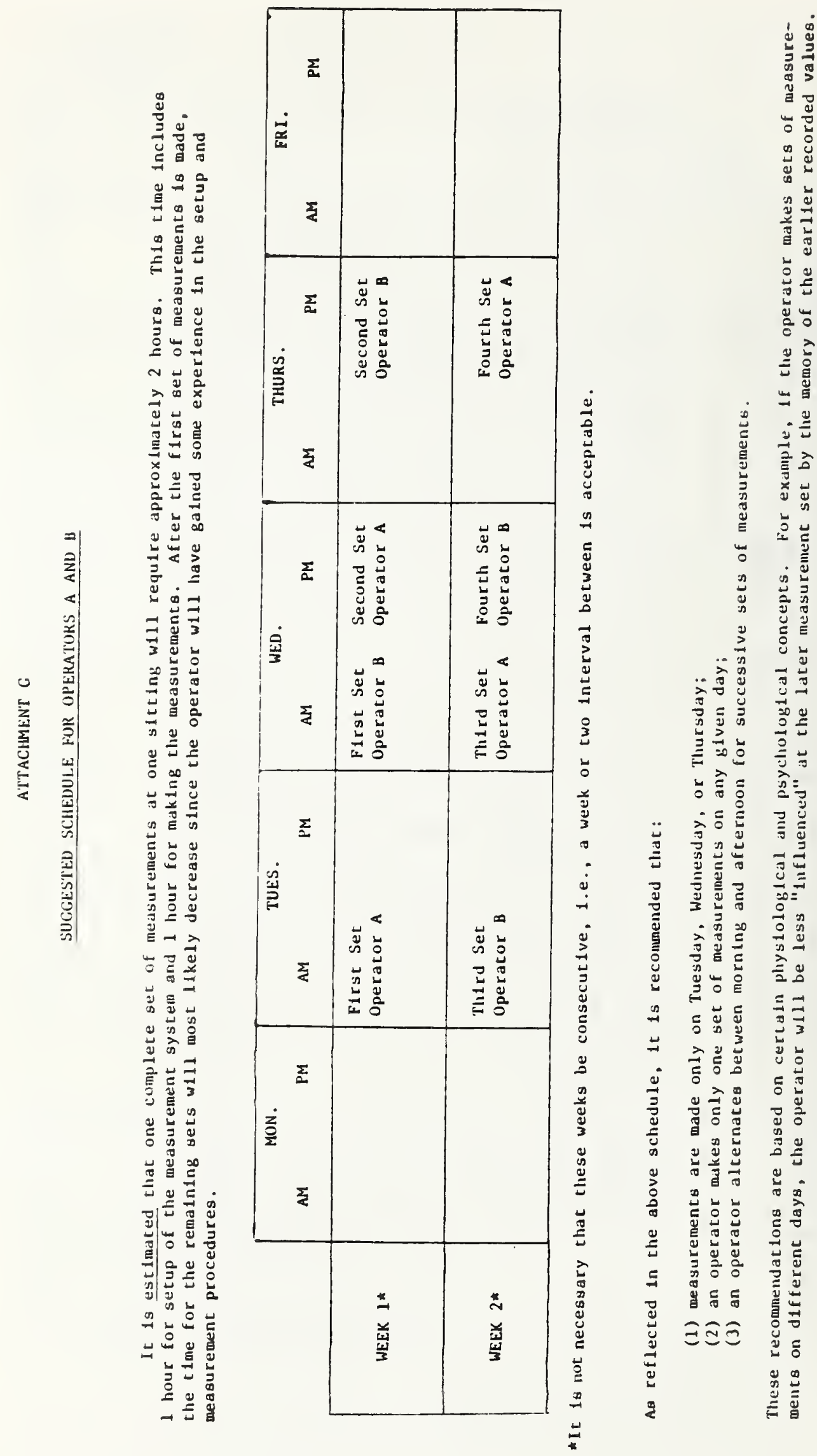




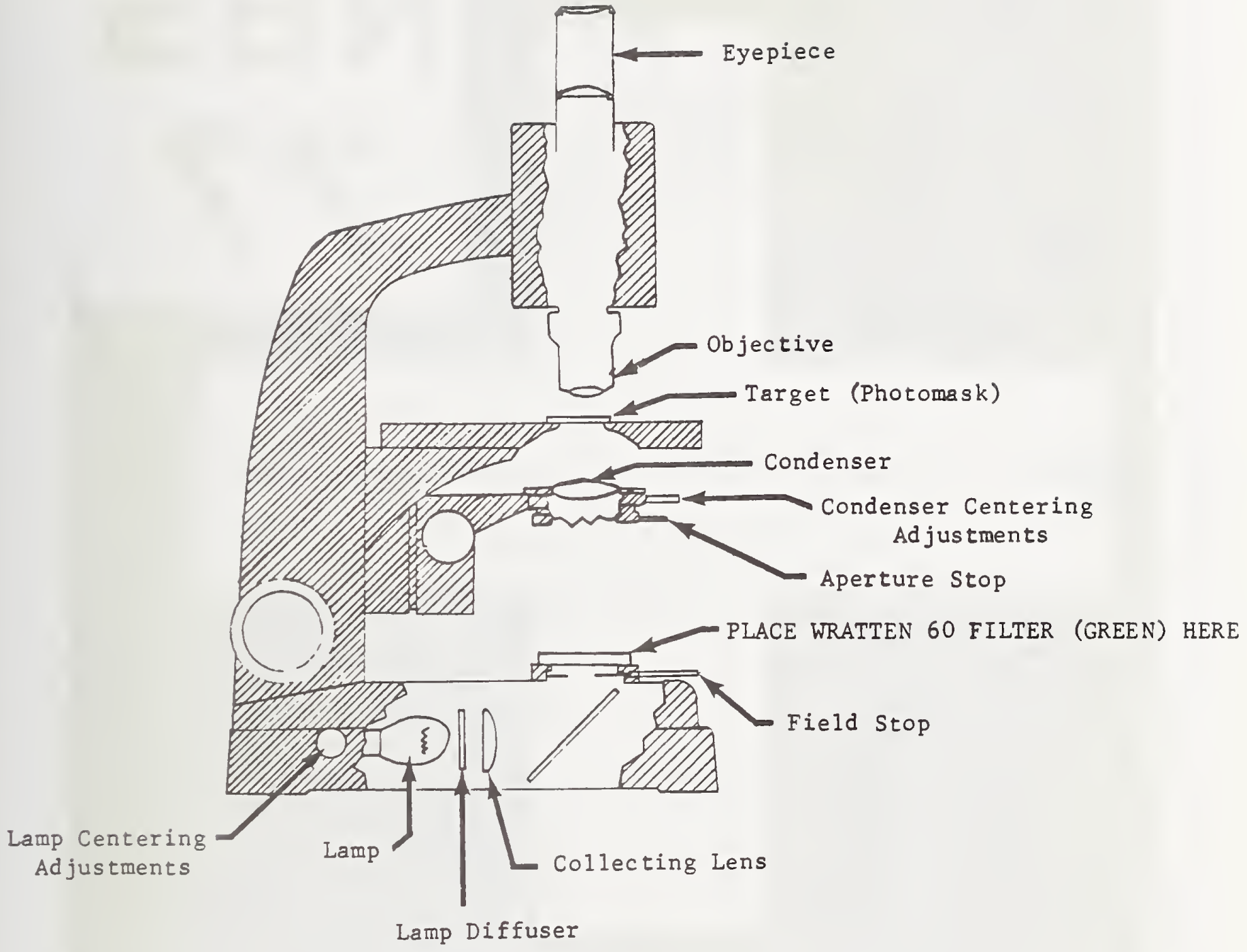

Figure 1. Schematic of an optical microscope. The labelled parts are defined and discussed in Attachment $A$. This schematic is not intended to represent the microscope of any particular manufacturer. The Wratten 60 filter is provided by NBS and should be placed at the location designated in this schematic. 


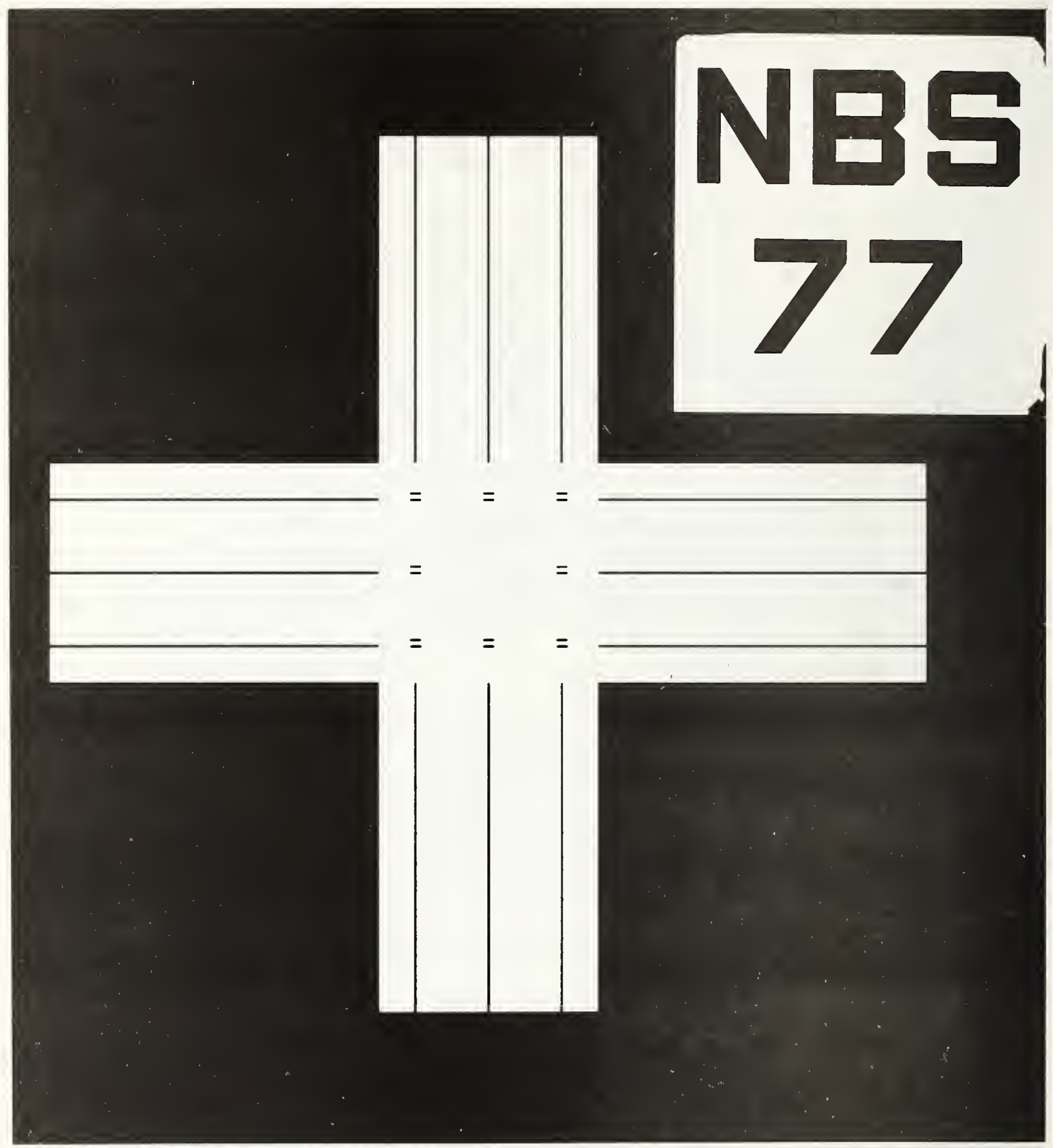

Figure 2. An overall view of the NBS target. The horizontal and vertical lines serve as an aide. for locating the elght pattern groups in the center of the target. 

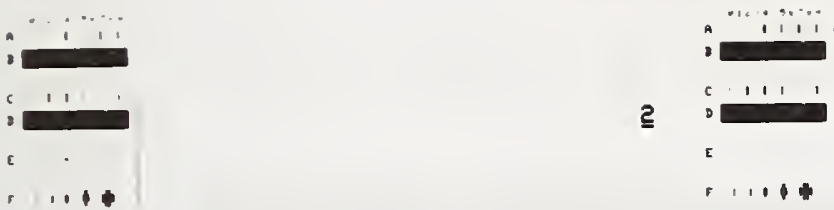

(
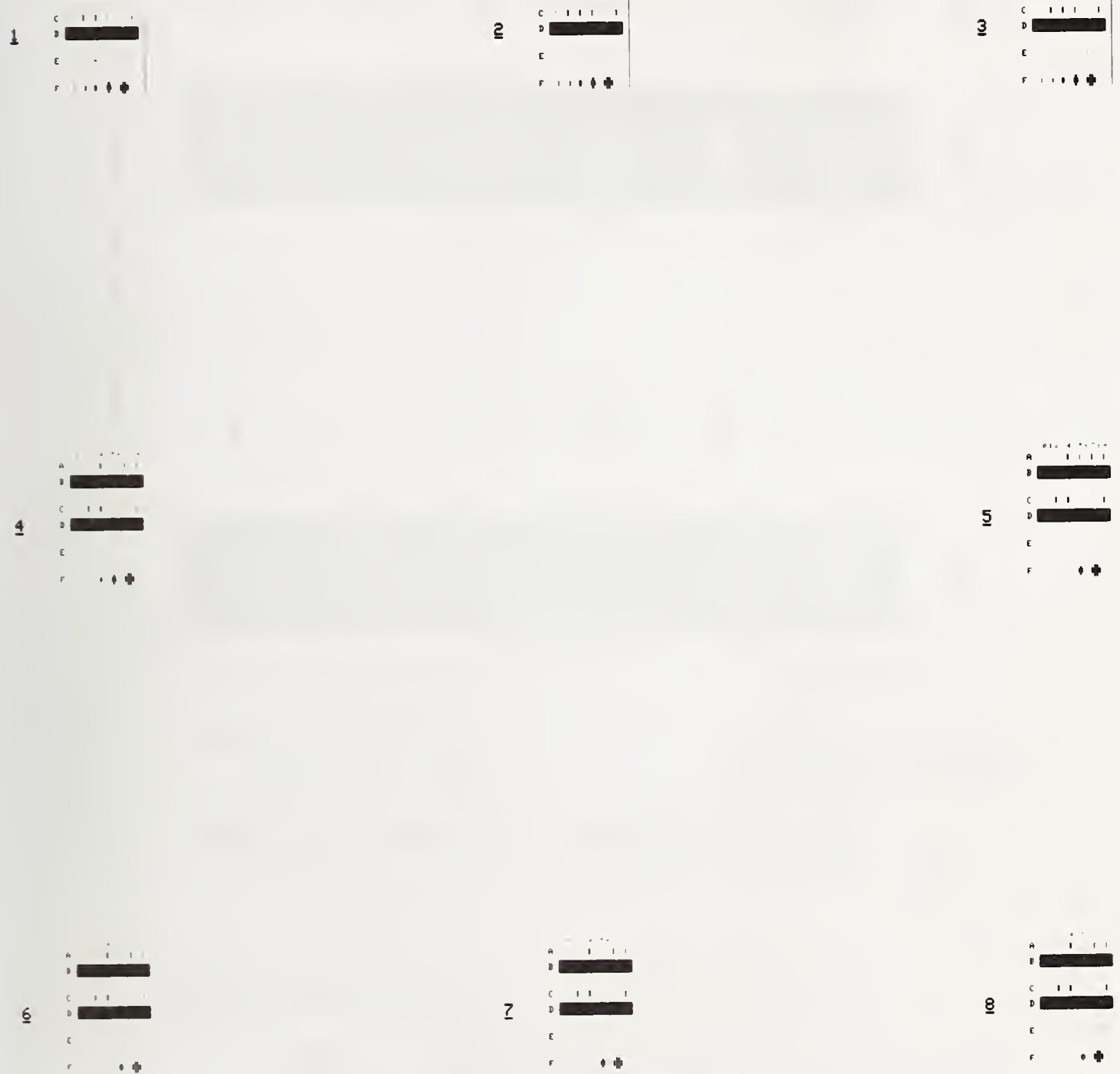

Figure 3. A view of the center of the NBS target. The basic pattern is repeated eight times, and each pattern group is numbered. (The specific pattern group on which you will make your measurements is identified in the instructions.) 


$$
\begin{aligned}
& \begin{array}{llllllllll}
0 & 1 & 2 & 3 & 4 & 5 & 6 & 7 & 8 & 9
\end{array} \\
& \text { A }-|-|-|-|-|--|-|-|-|-|- \\
& \text { B }-|-|-|-|-1-1-1-1-1- \\
& c-|-|-|-|-|--|-|-|-|-|- \\
& \text { D }-1-1-|-|-1--|-|-\mid-1-1=
\end{aligned}
$$
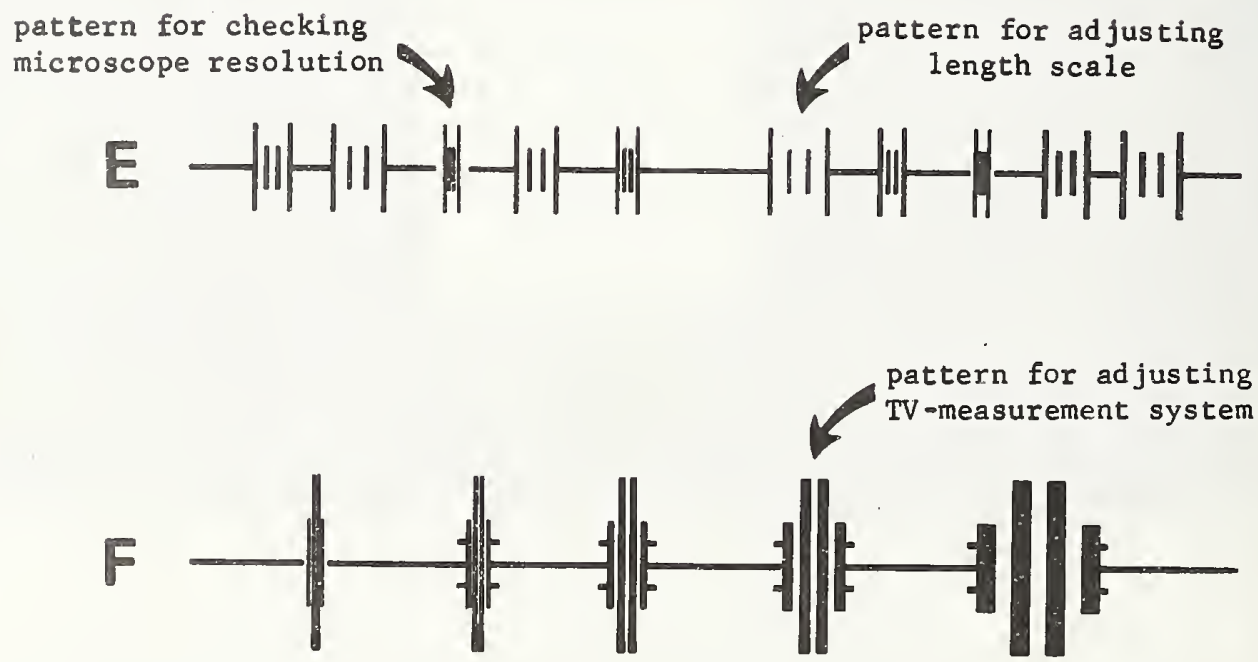

Figure 4. A view of the basic pattern group on the NBS target. The lines and line-spacings to be measured are located by reference to an alphanumeric grid with the letters A through $\mathrm{F}$ identifying the row and the numbers 0 through 9 identifying the column. The pattern E-2 is used to check the resolution of the microscope objective. The pattern E-5 is used to adjust the measurement system for the NBS-furnished length scale. The pattern F-5 is used for adjusting the ratio of a linewidth to the width of an adjacent space on TV-measurement systems. 


\section{$\begin{array}{llllll}0 & 1 & 2 & 3 & 4 & 5\end{array}$}

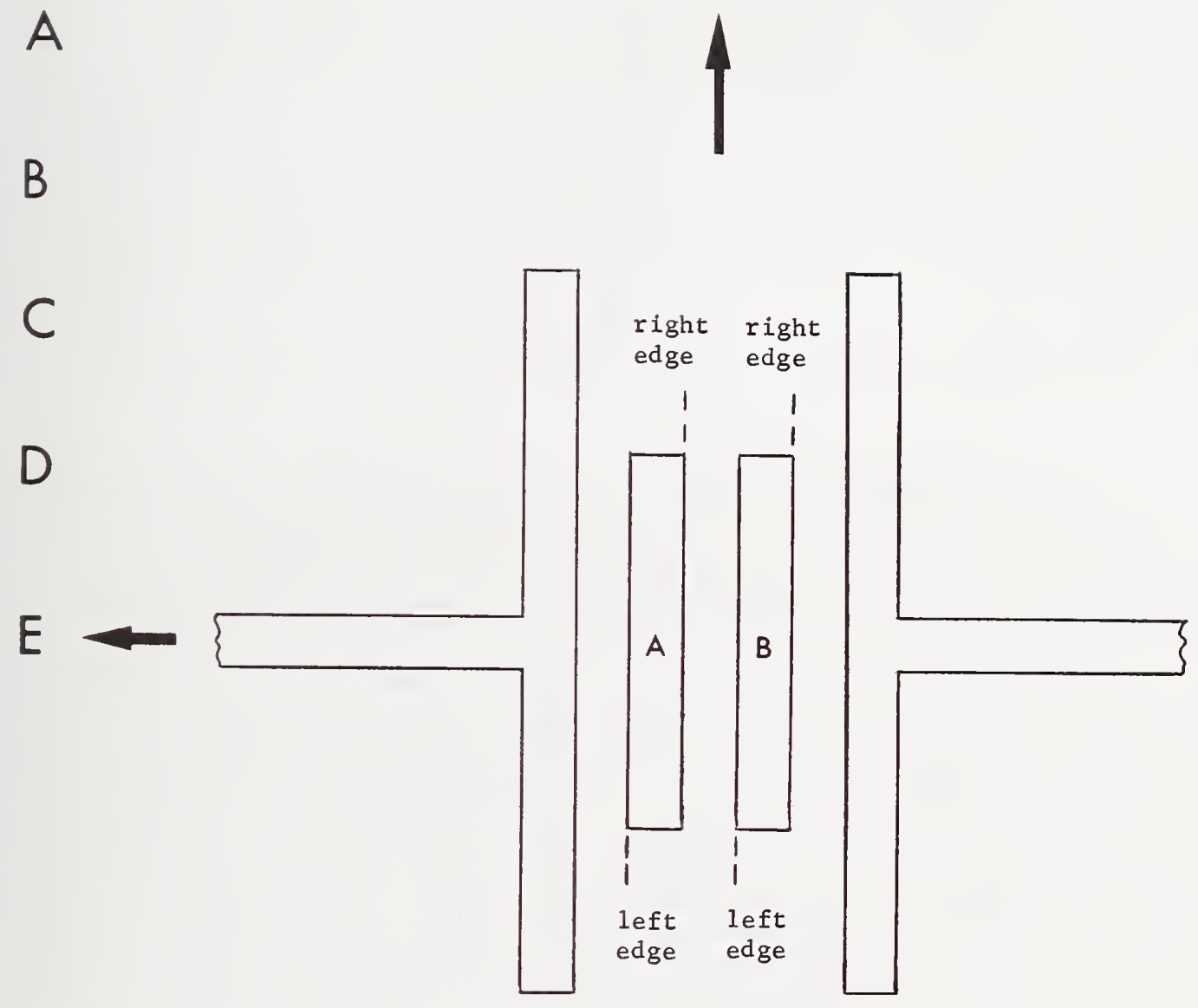

Figure 5. Four-line pattern for pitch measurements. These patterns are E-0 through E-9 on the target. Pitch measurements are made only on the interior line pair (A and $B$ ). The specific pattern represented here is E-5 which is used to adjust the measurement system or correct the data for the NBS-furnished length scale. Note the orientation of the left edges of lines $A$ and $B$ with reference to the letters and numbers that mark the row and column location. 


\section{$\begin{array}{llllll}0 & 1 & 2 & 3 & 4 & 5\end{array}$}

A

B

C

D
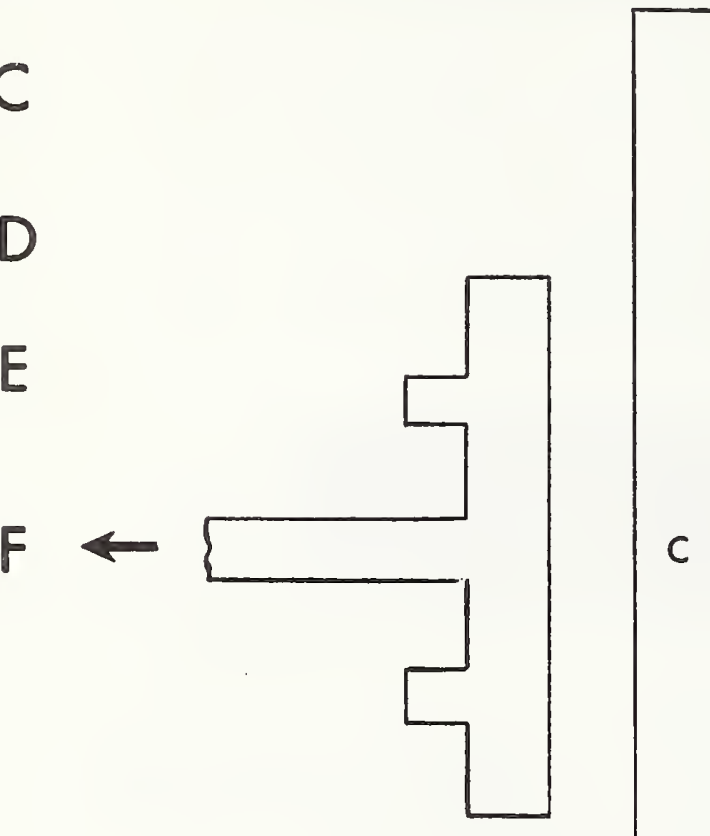

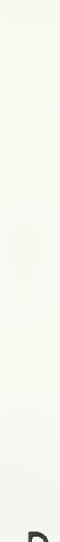

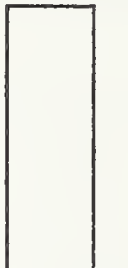

\begin{tabular}{l|}
$|C|$ \\
$\mid$
\end{tabular}
left
edge
left
edge

$\begin{aligned} & \text { C } \\ & \mid\end{aligned}$
$\mid$
left
edge
left
edge

\section{$\uparrow$}

Figure 6. Four-line pattern for adjusting the LINE-SPACE control on TV measurement systems. The NBS value for the ratio of the width of line $C$ to the width of space $D$ is used for this adjustment. The letters and numbers, which mark the row and column location of the pattern, are shown to help identify the left edges of line $C$ and space $D$. 


\section{APPENDIX D}

\section{Participants' Linewidth and Line-Spacing Measurements}

The raw data from the participants in the study are given in table $\mathrm{D}-1$. This table consists of 705 lines of information representing the twenty measurement systems included in the study. Data from some companies or groups within a company that did not follow instructions or did not furnish requested data are not included in this table. (See table 2, sec. 2.4.) In each case where the participant furnished all the requested measurements on one measurement system there are forty lines of information; eight of these lines represent the four sets of independent measurements made by each of two operators for each measured linewidth or line spacing on the artifact. In those cases where the participants did not furnish all of the requested data, there are less than forty lines of information. The data are sorted by company/ measurement system, row on the artifact ( $A, B, C, D$, or E), operator, and chronological order of the measurements. Any obvious transcription error in the raw data was corrected, and the corrected value is included in table $\mathrm{D}-1$.

Each line in the data listing contains the following information: ten measurements; a character $A, B, C, D$, or $E$ denoting the row measured on the artifact; a seven-character code uniquely identifying each line of data; a conversion factor for converting the data to micrometers; and a set of ten digits denoting the presence or absence of outliers in the data. The ten measurements are recorded from left to right in accordance with the pattern on the artifact; for example; A-0 thru A-9 on row A. (See fig. 3, sec. 2.1.) The first three characters of the code are used to identify the measurement system. (See table 2, sec. 2.4.) The fourth and fifth characters of the code are the initials of the operator. The sixth character denotes the chronological order of the data. The seventh character designates the units of the data; (I - microinch, M - micrometer, and A - arbitrary units). For example, the first three lines of the listing represent the three sets of measurements on the ten opaque lines in row $A$ made by the first operator with initials $\mathrm{BB}$ for the measurement system designated as $1 \mathrm{I}$.

The set of ten digits used to denote outliers in the data corresponds to the ten measurements on a row. Each digit has a value of 0,1 , or 2 . A value of 1 denotes that the measurement is included in the data analysis. A value of 0 denotes that the measurement is considered to be an outlier and is not used in the analysis. Two of the recorded measurements are considered to be transcription errors and are also flagged with a zero. (See lines with codes C 7CMB1M and C10INO2M.) In cases where the ten digits are all two's, an entire day's measurements are considered outliers. (See sec.3.3.2.)

Table D-2 gives linewidth data for rows $C$ and $D$ that have been corrected based on linewidth calibration curves derived from rows $A$ and $B$, respectively. In some cases, these linewidth data have also been corrected based on a linespacing calibration curve derived from row $E$. The data for rows $A$ and $B$ in this table are the same data as that given in table D-1 except for the cases in which a line-spacing correction was applied. (See table 4.) The format for data in table $\mathrm{D}-2$ is the same as that described for data in table $\mathrm{D}-1$. 


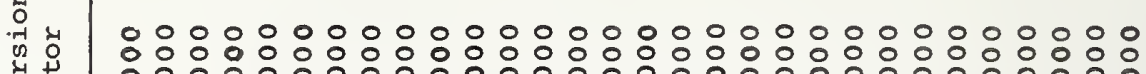
Uు

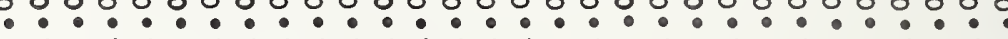
$\sum \sum \sum \sum \sum \sum \sum \sum \sum \sum \sum \sum \sum \sum \sum_{M} \sum \sum \sum \sum \sum \sum_{M} \sum \sum \sum \sum \sum_{M} \sum_{M} \sum \sum \sum_{M}$

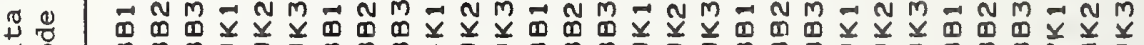
峁

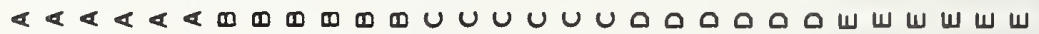

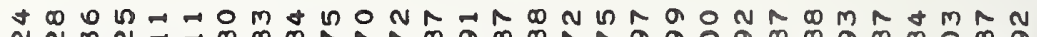

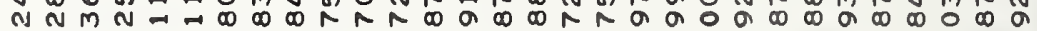

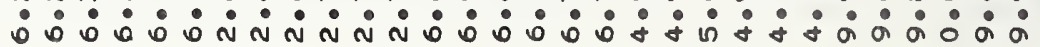

옹

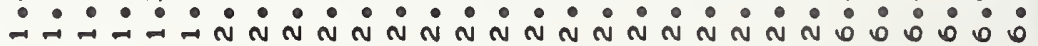

* + a n

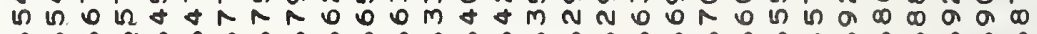

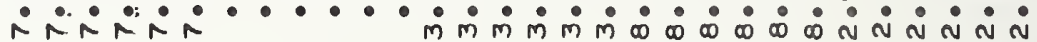

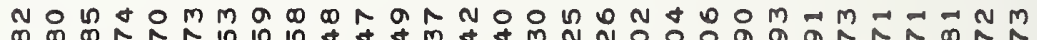

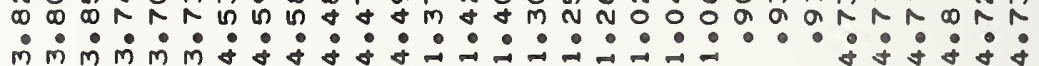

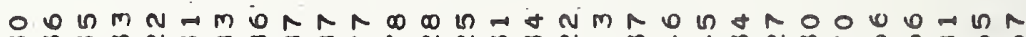
$M M M M N M \infty \infty \infty \infty R N N N M M N \rightarrow M+\forall M N M R 00000$ i

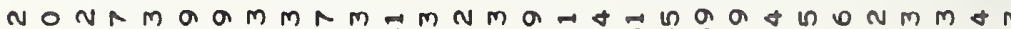

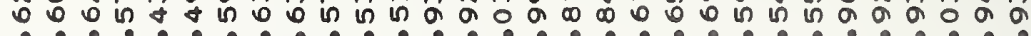

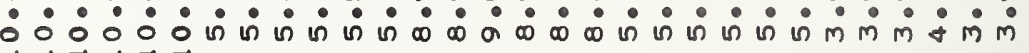

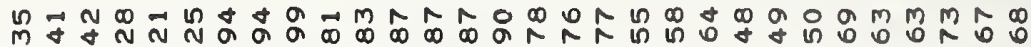

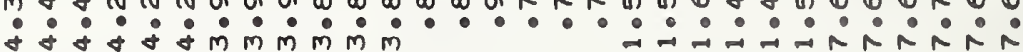

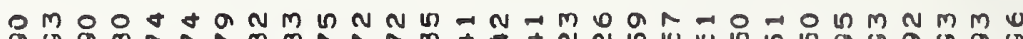
:

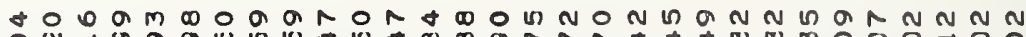
(v)? a

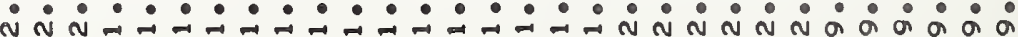

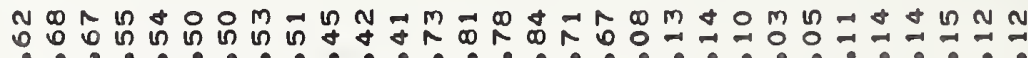

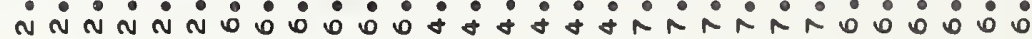


0000000000000000000000000000000000000000

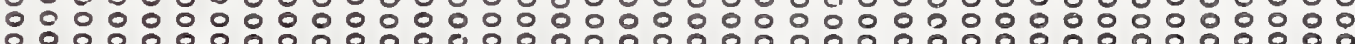

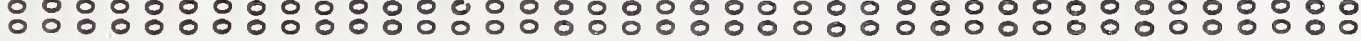

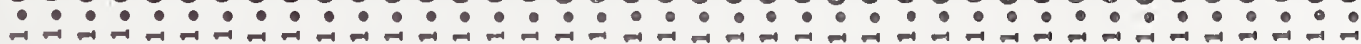

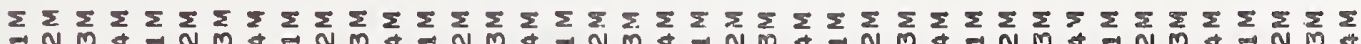

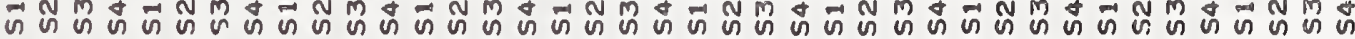

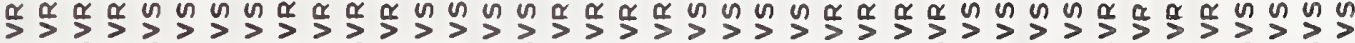

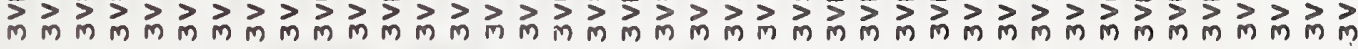

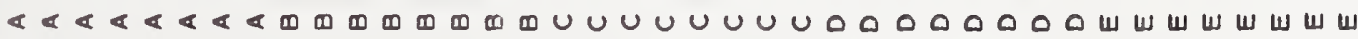

○N゚

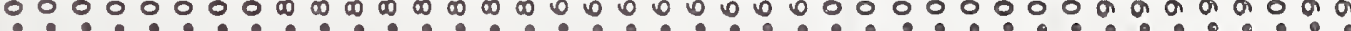

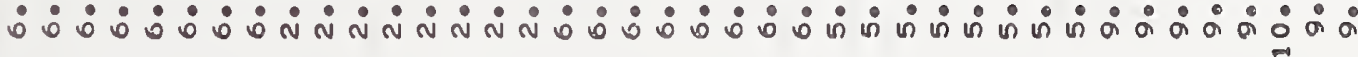

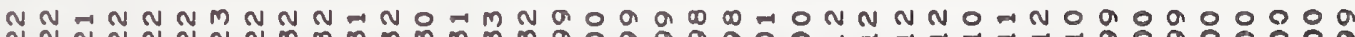
‥ ㄴ.?

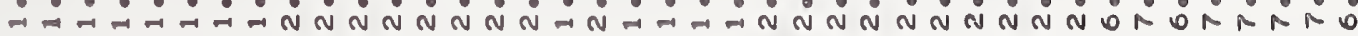

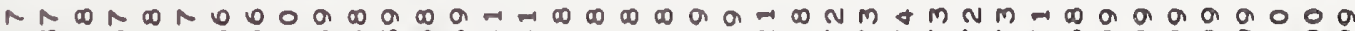
mmmmmmmmo n

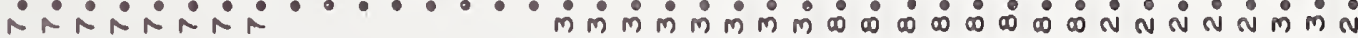

$\overrightarrow{0} \overrightarrow{0} \overrightarrow{0} \overrightarrow{0} \overrightarrow{0} 0 \overrightarrow{0} 0$ 유

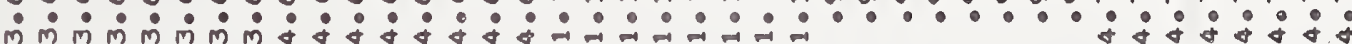

ONOOMNNOmmmmNmOO

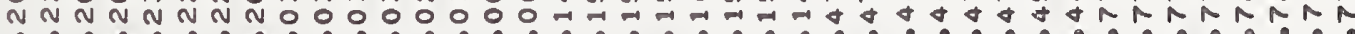
ம் ம் ம் ம் ம்-

m

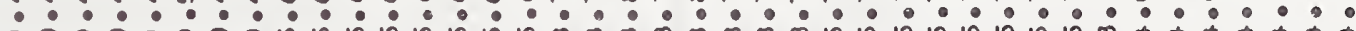
○0 00000 ம

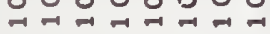

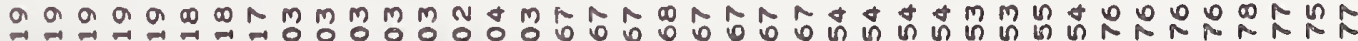

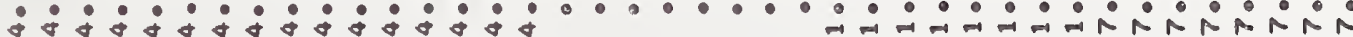

눈

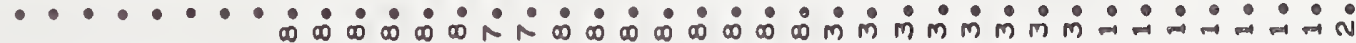

0 n $n$ n

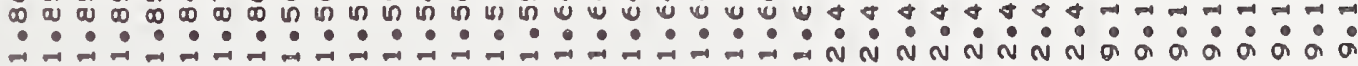

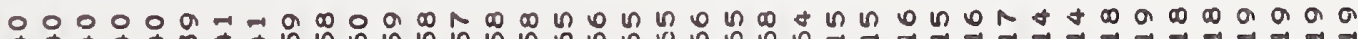

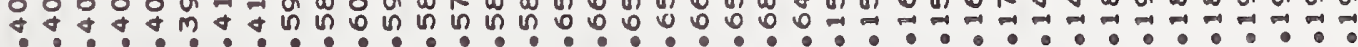

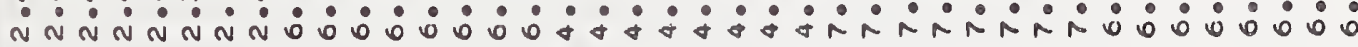




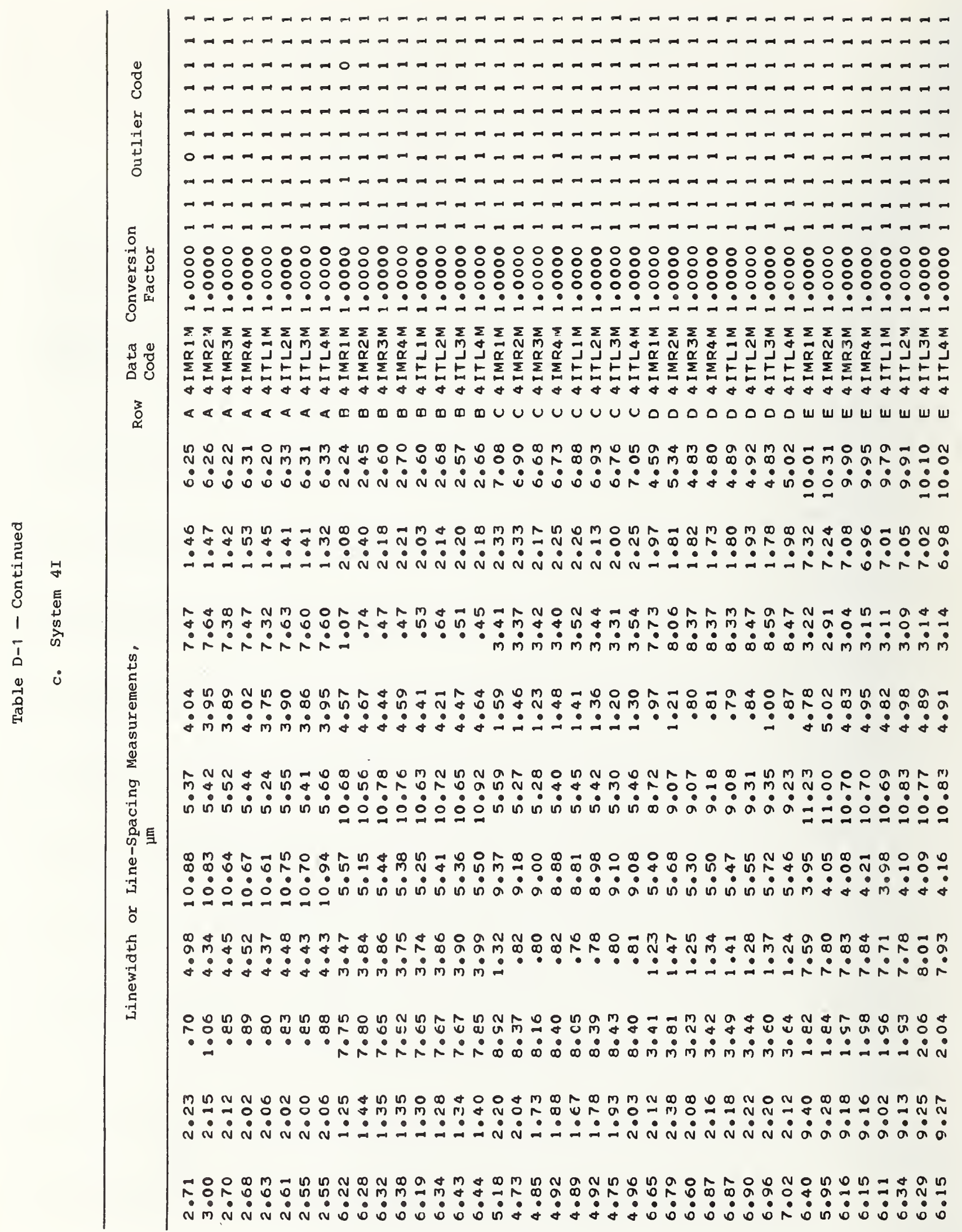




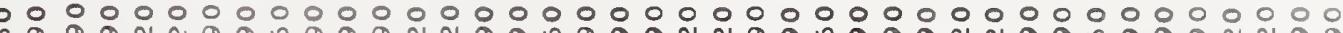
ra na a

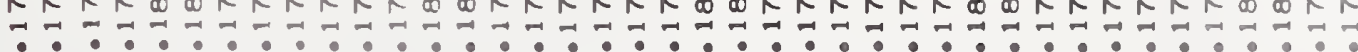

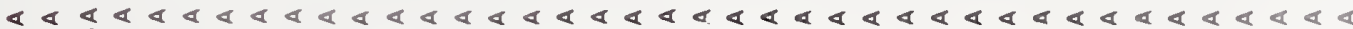

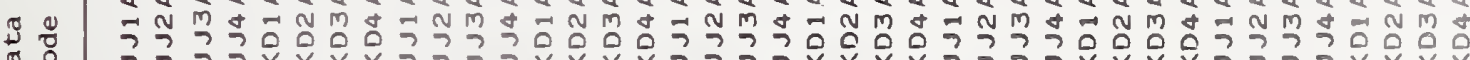

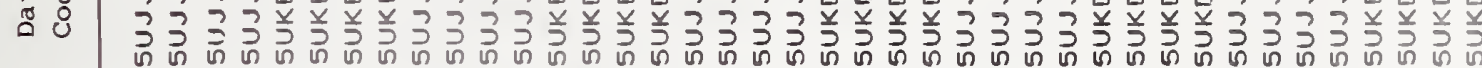
ב⿳亠口冋

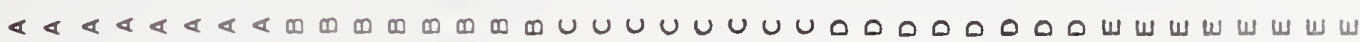

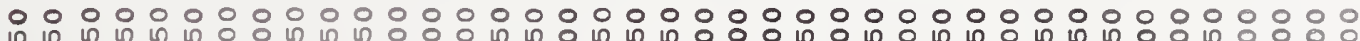

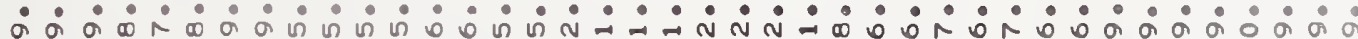

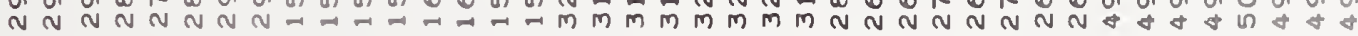

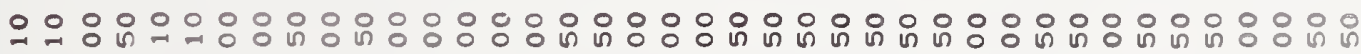

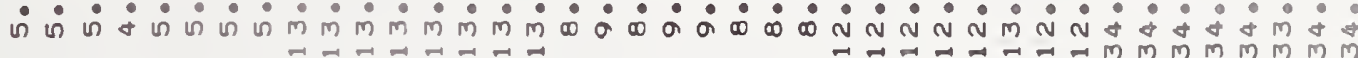

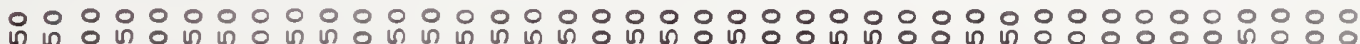

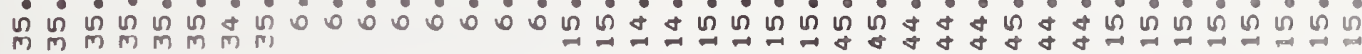

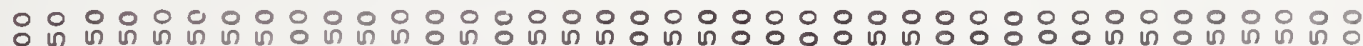

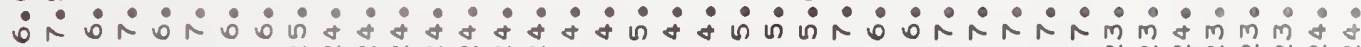

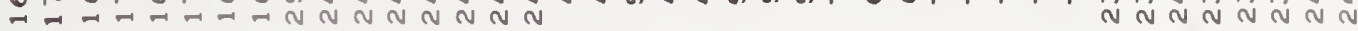

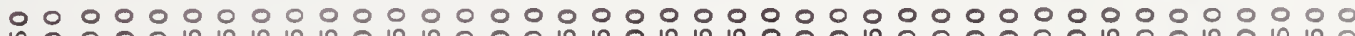

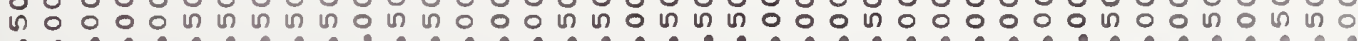

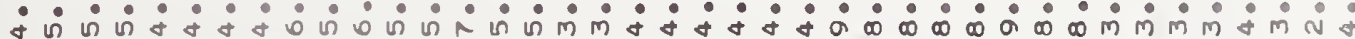
a $N$ N $N$ N

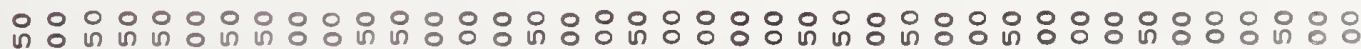
$\dot{0} \dot{0} \dot{0} \dot{0} \dot{0} \dot{0} \dot{0} \dot{0} \dot{i} \dot{i} \dot{\sim} \dot{\sim} \dot{\sim} \dot{i} \dot{0} \dot{0} \dot{0} \dot{0} \dot{0} \dot{0} \dot{0} \dot{0} \dot{0}$

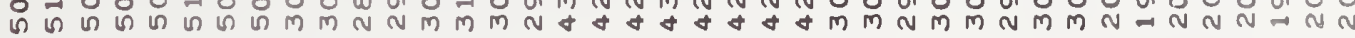

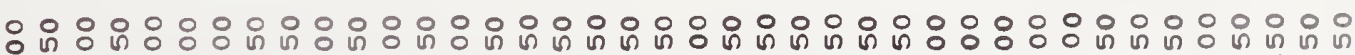

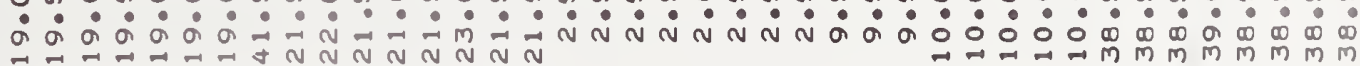

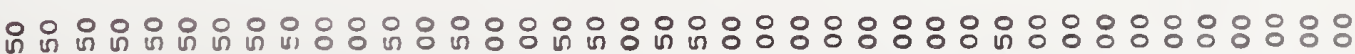

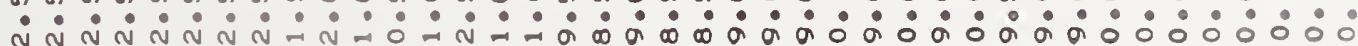

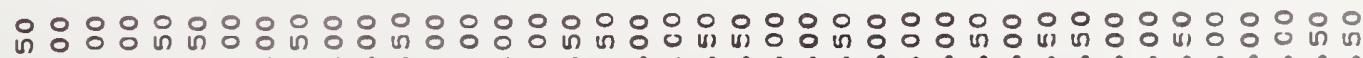

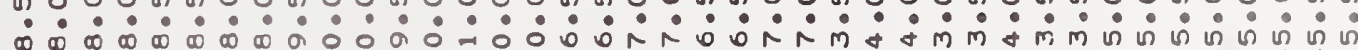

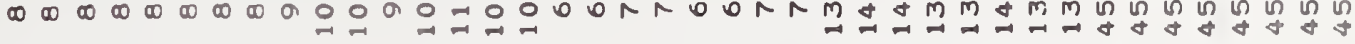

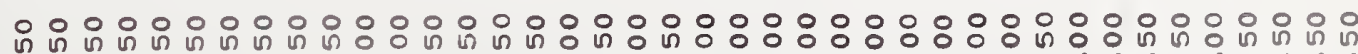

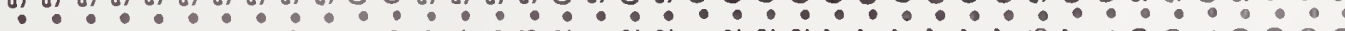

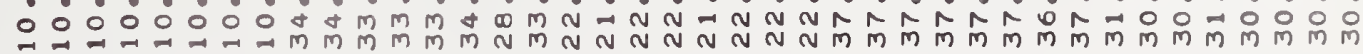




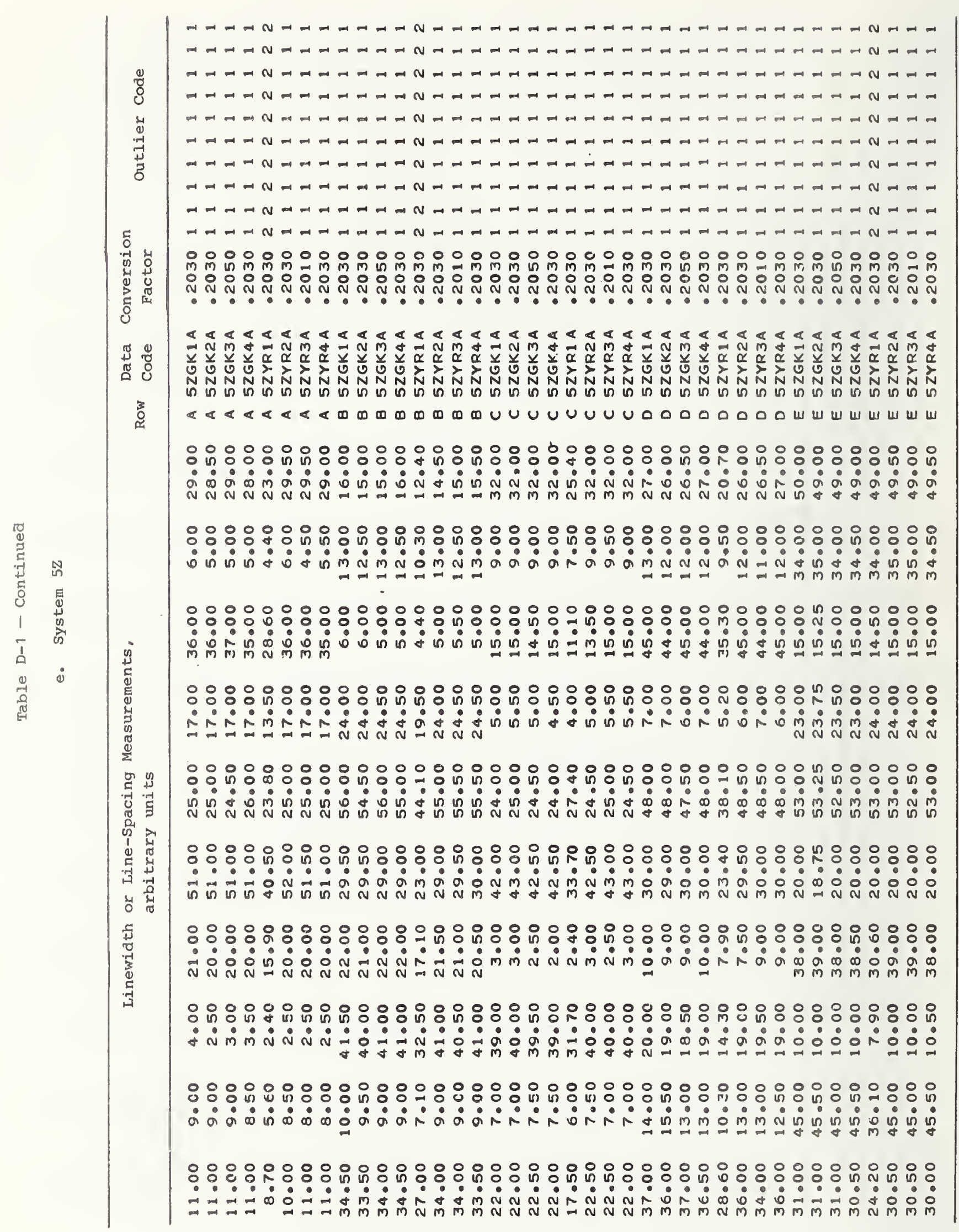




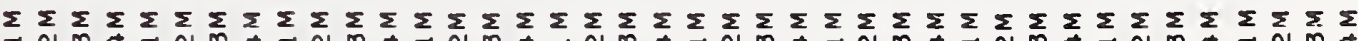
Iำ

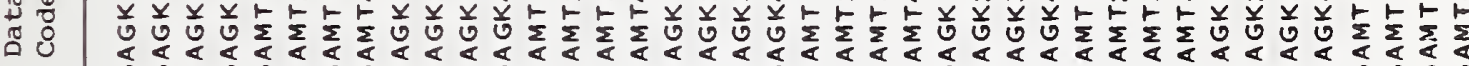

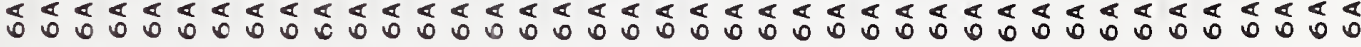

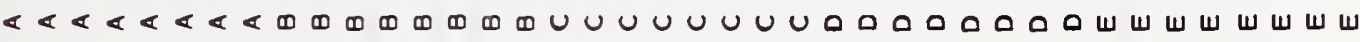

- no na O O O MO N $\dot{0} \dot{0} \dot{0} \dot{0} \dot{0} \dot{0} \dot{m} \dot{m} \dot{m} \dot{m} \dot{m} \dot{0} \dot{0} \dot{0} \dot{0} \dot{0} \dot{0} \dot{0} \dot{0}$ ம

N ก. ค กา

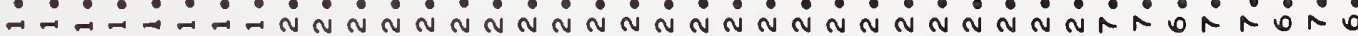

NGMDN

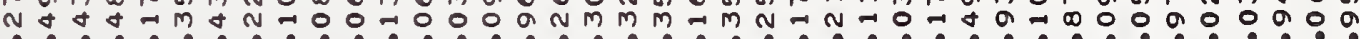

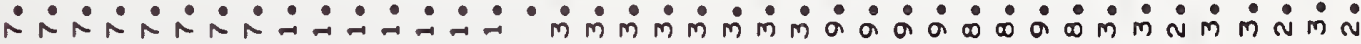

nก ? ? ? ? ? ? ? ? ? ? ? ? ? ? ? ? ? : ? ? ?

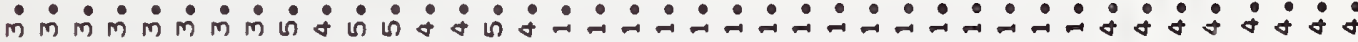

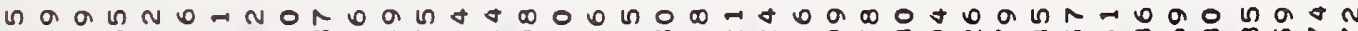

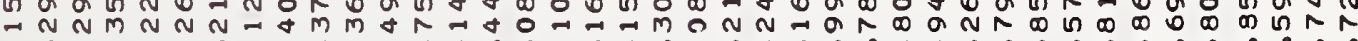

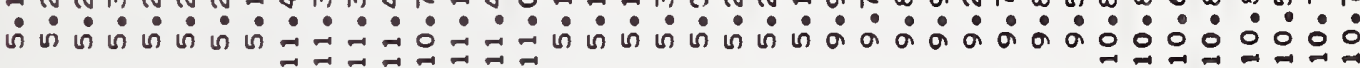

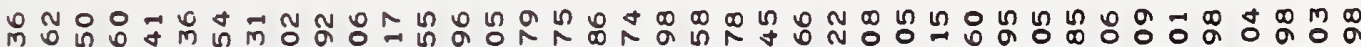

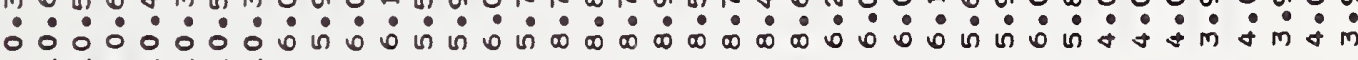

๑

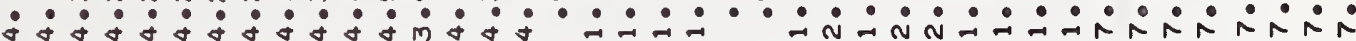

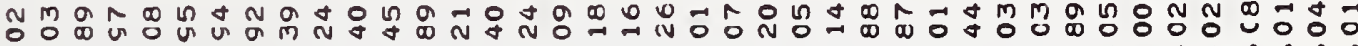

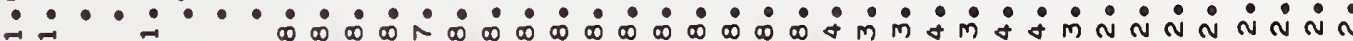

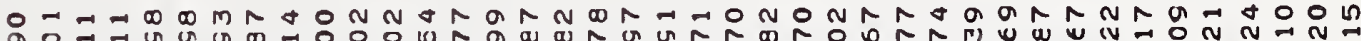

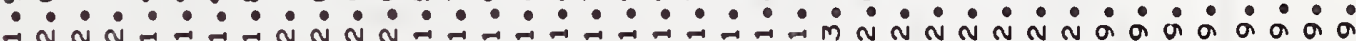

人 n M N

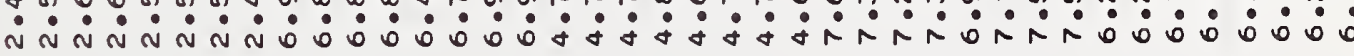




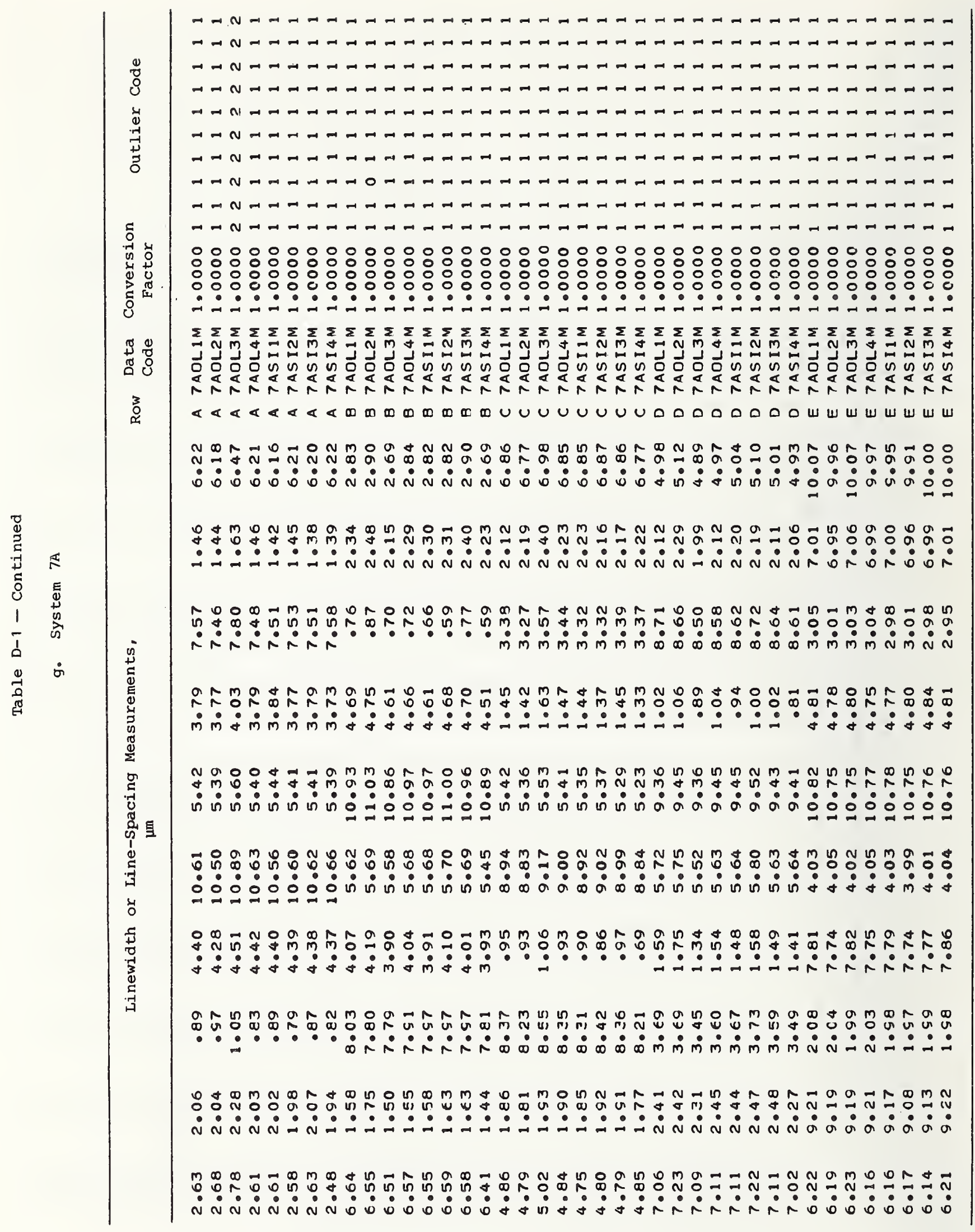




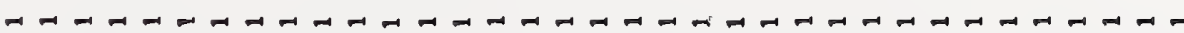

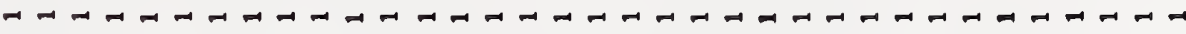

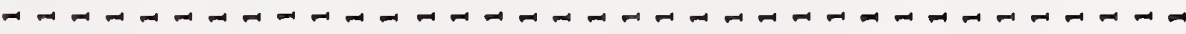

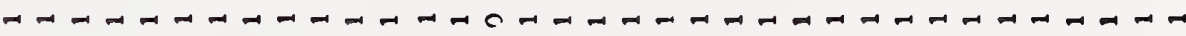

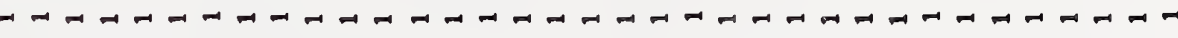

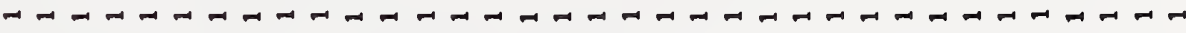
ન-

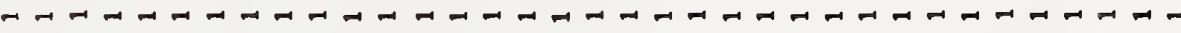

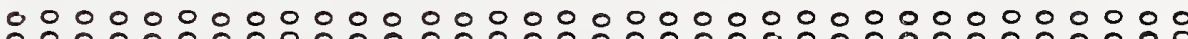

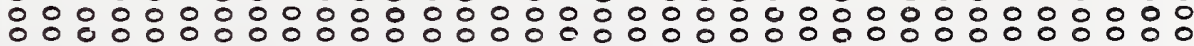
000000000000000000000000000000000000

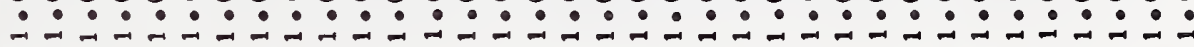

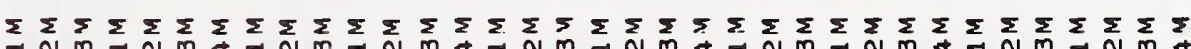

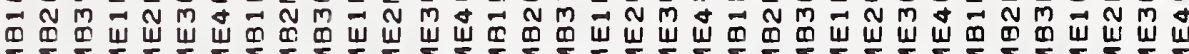

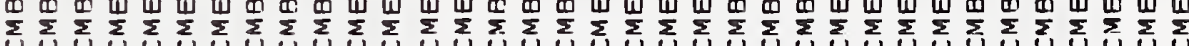

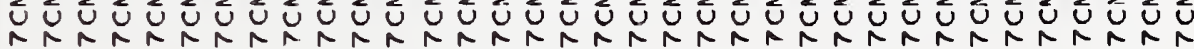

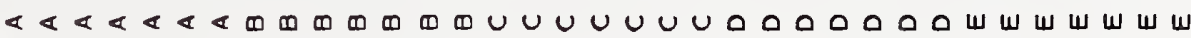

ㄱำ

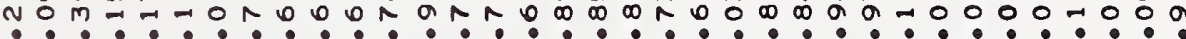

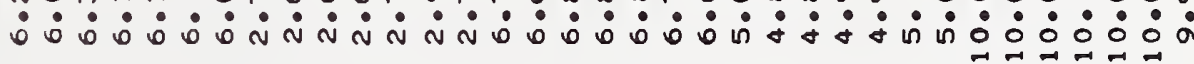

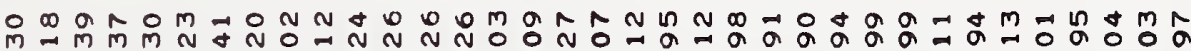

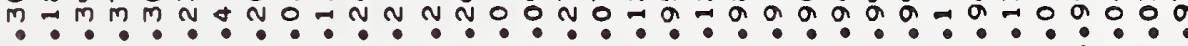

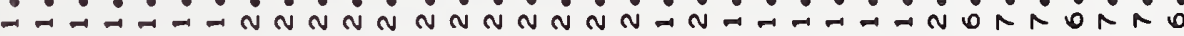

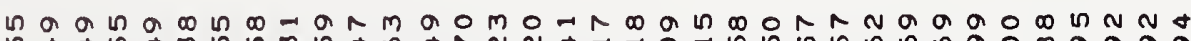
n?

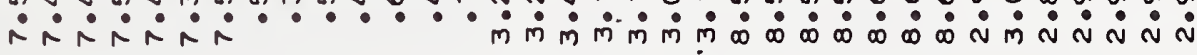

Nลำคm

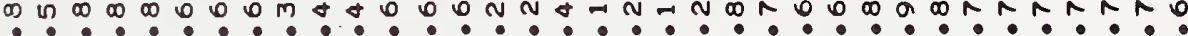

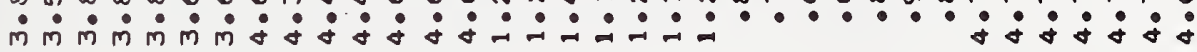

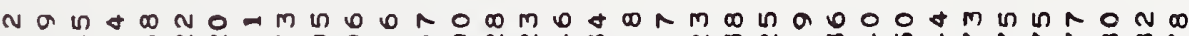

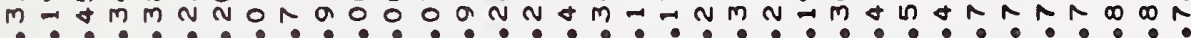

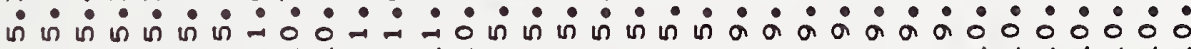

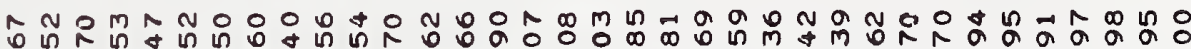

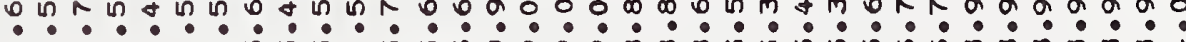

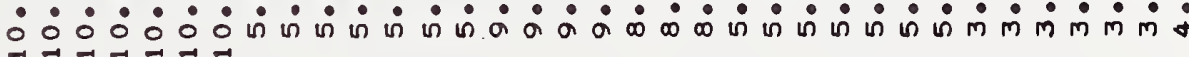
エ

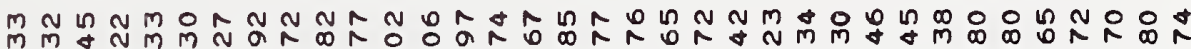
$m m \& n m m$ m

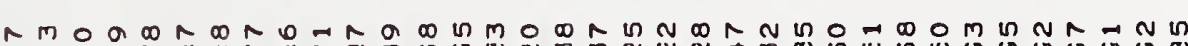

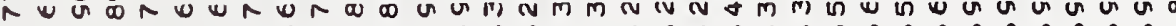

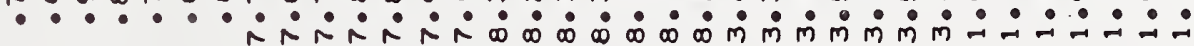

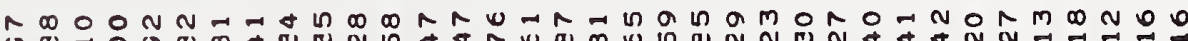
แ

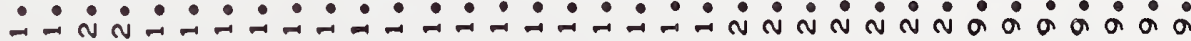

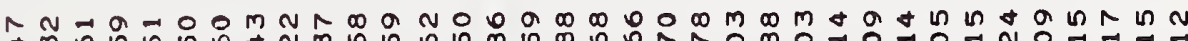

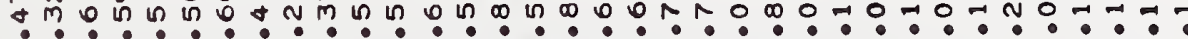

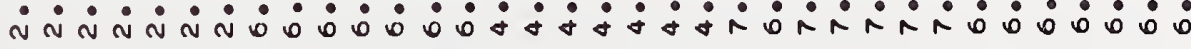




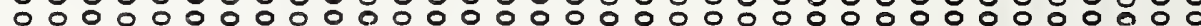
00000000000000000000000000000000000

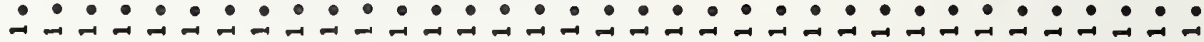

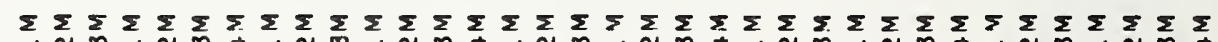

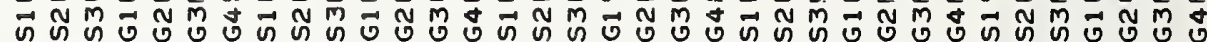

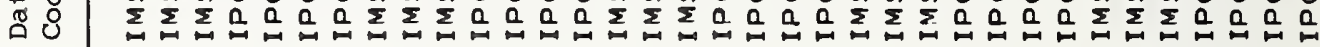

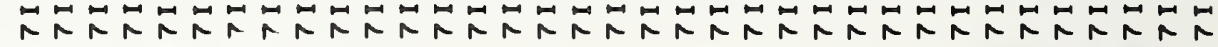

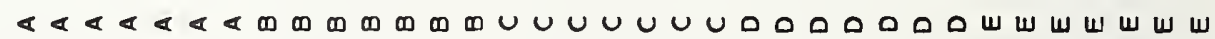

a n $\dot{0} \dot{0} \dot{0} \dot{0} \dot{0} \dot{N} \dot{N} \dot{N} \dot{N} \dot{N} \dot{N} \dot{\sim} \dot{\sim} \dot{0} \dot{0} \dot{\sim} \dot{q} \dot{q} \dot{q} \dot{+} \dot{j} \dot{0} \dot{0} \dot{0} \dot{0} \dot{0}$

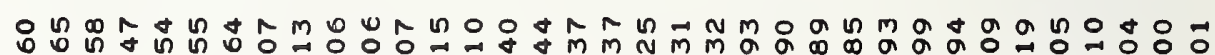
:

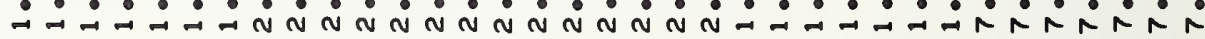

nno a

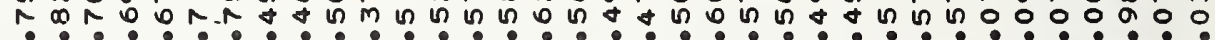

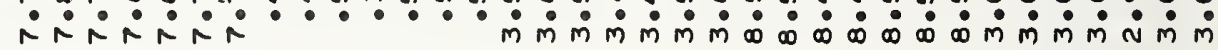

次

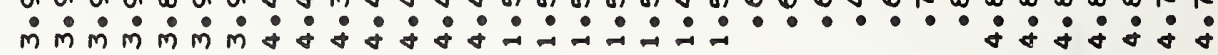
영

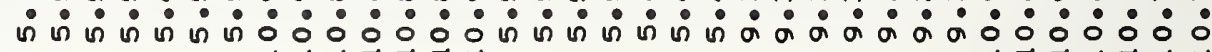

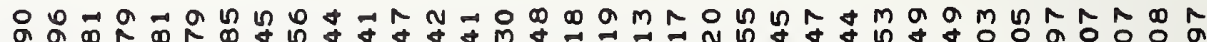
? $\therefore \circ: 000$ ก $\rightarrow \rightarrow \rightarrow-1=$

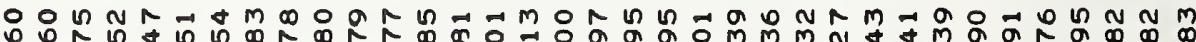
? ? ? ? : $?$ ?

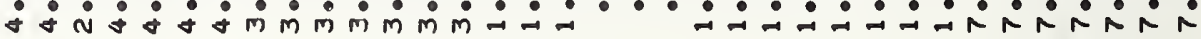

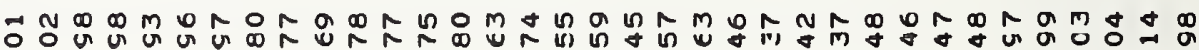

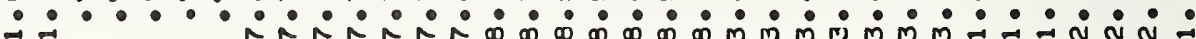
N $\dot{N} \dot{N} \dot{N} \dot{N} \dot{N} \dot{N} \dot{-} \dot{\sim} \dot{-} \dot{-} \dot{\sim} \dot{N} \dot{N} \dot{\sim} \dot{\rightarrow} \dot{\sim} \dot{N} \dot{N} \dot{N} \dot{N} \dot{N} \dot{N}$ a

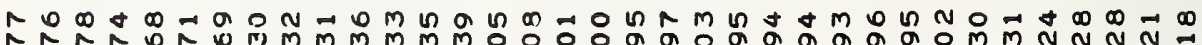

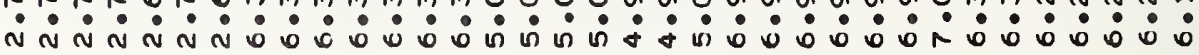




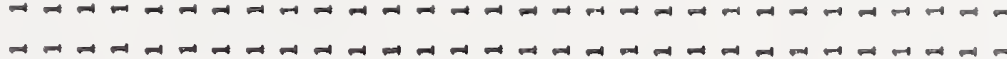

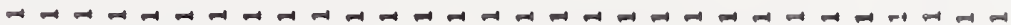

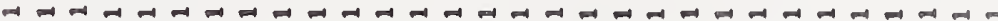

•नન-

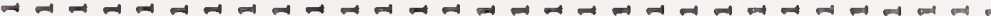

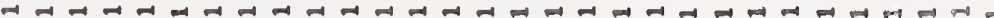

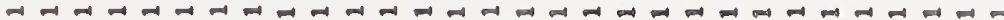
(1) N N N N N N N N N N N N N N N N N N N N N N N N N N N N N N

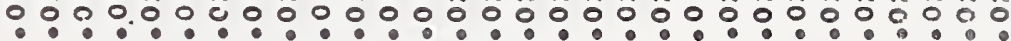

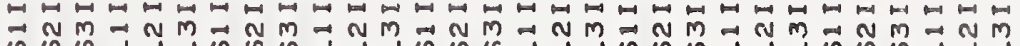

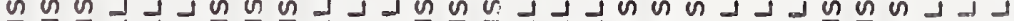

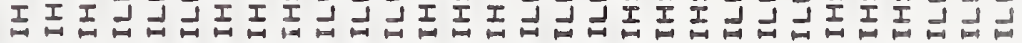

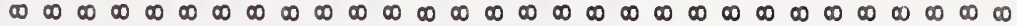

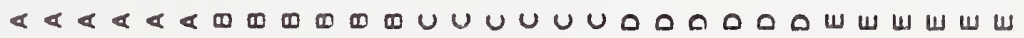

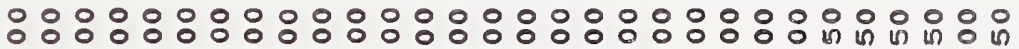

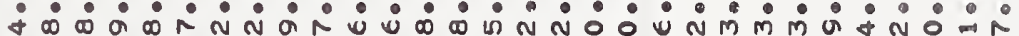

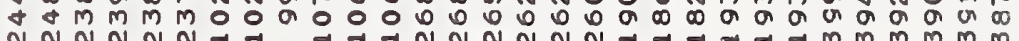
ㅇㅇㅇㅇㅇㅇㅇㅇㅇㅇㅇㅇㅇㅇㅇㅇㅇㅇㅇㅇㅇㅇㅇㅇㅇㅇㅇㅇㅇㅇㅇㅇㅇㅇㅇ응

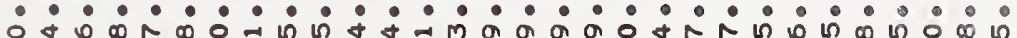

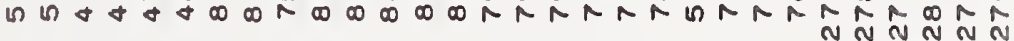

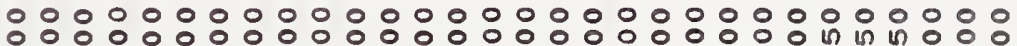

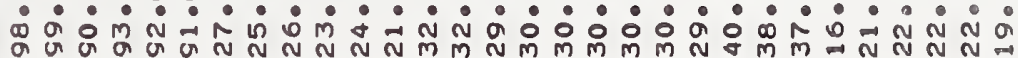
$N \mathbb{N} N \mathbb{N} N N_{-1}$

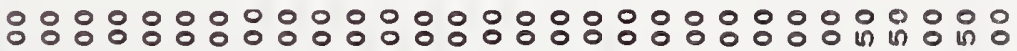

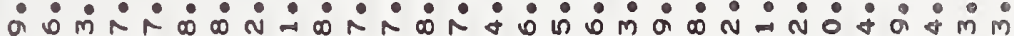

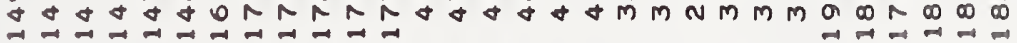

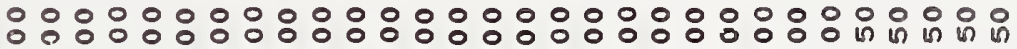
$\dot{0} \dot{0} \dot{0} \dot{0} \dot{0} \dot{0} \dot{0} \dot{0} \dot{0} \dot{0} \dot{0} \dot{0} \dot{0} \dot{0} \dot{0} \dot{0} \dot{0} \dot{0} \dot{0}$ N 응ㅇㅇㅇㅇㅇㅇㅇㅇㅇㅇㅇㅇㅇㅇㅇㅇㅇㅇㅇㅇㅇㅇㅇㅇㅇㅇㅇㅇㅇㅇㅇㅇㅇㅇㅇㅇㅇㅇㅇ $\therefore \dot{0} \dot{0} \dot{0} \dot{0} \dot{0} \dot{0} \dot{0} \dot{0} \dot{0} \dot{0} \dot{0} \dot{0} \dot{0} \dot{0} \dot{0}$

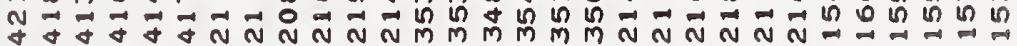

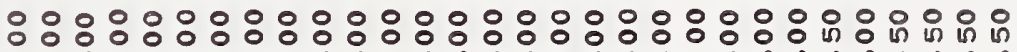
$-\dot{0} \dot{0} \dot{0} \dot{0} \dot{0} \dot{0} \dot{0} \dot{0} \dot{0} \dot{0} \dot{0} \dot{0} \dot{0} \dot{0} \dot{0} \dot{0}$

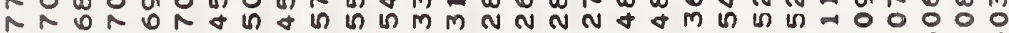

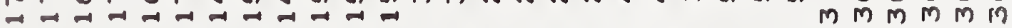
: : : : : : : : : : : : : : : : : : : : : :

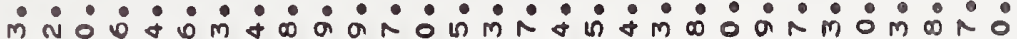

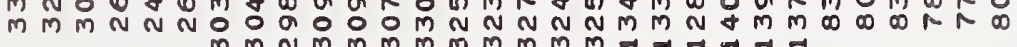

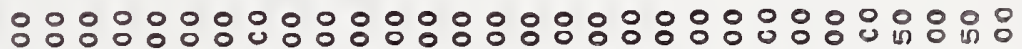

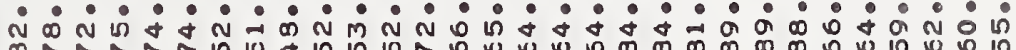

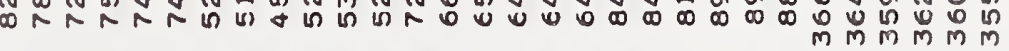

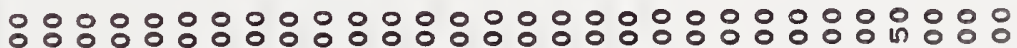
:

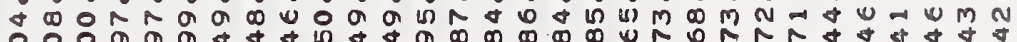
음 a a a 


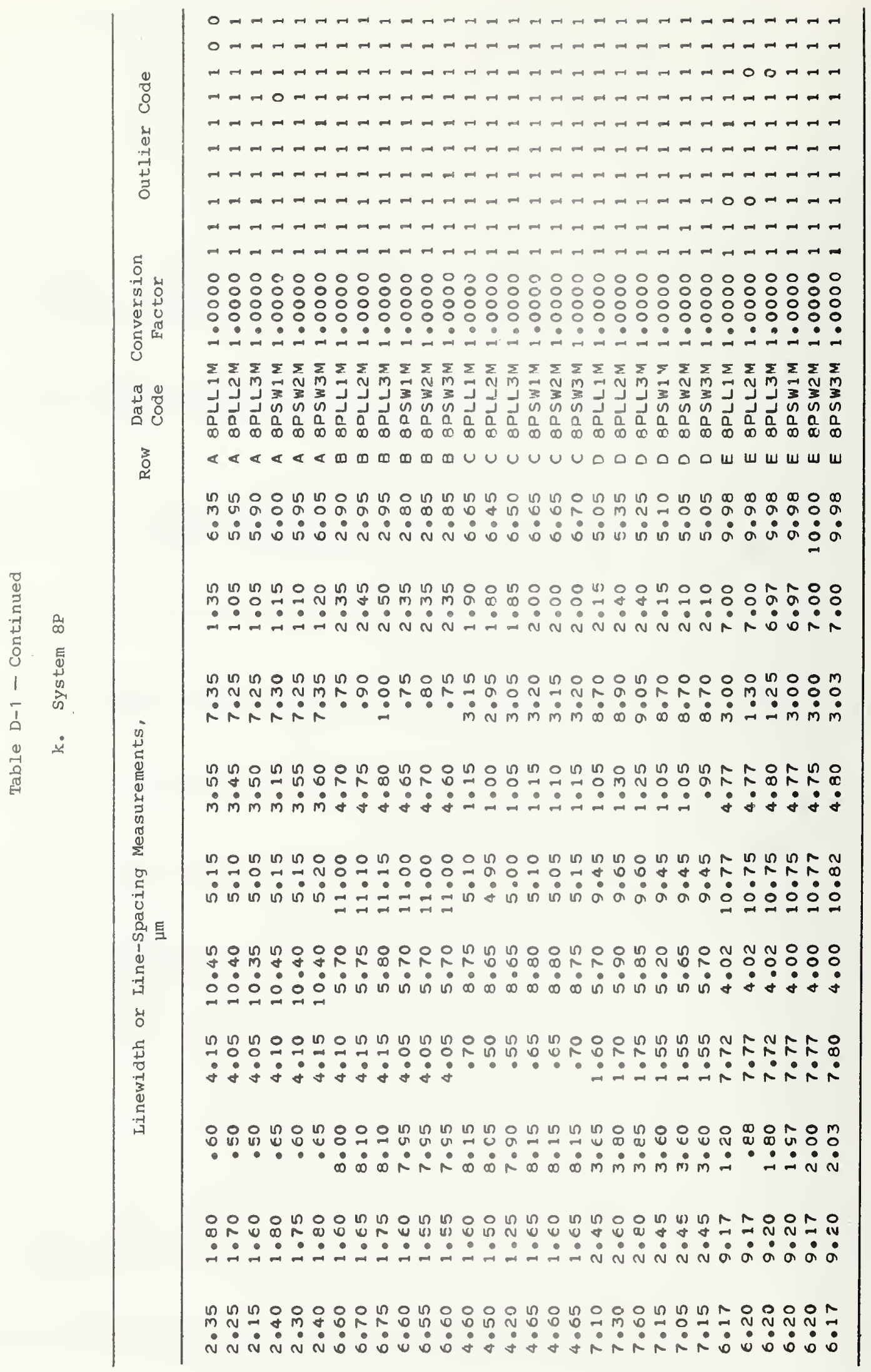




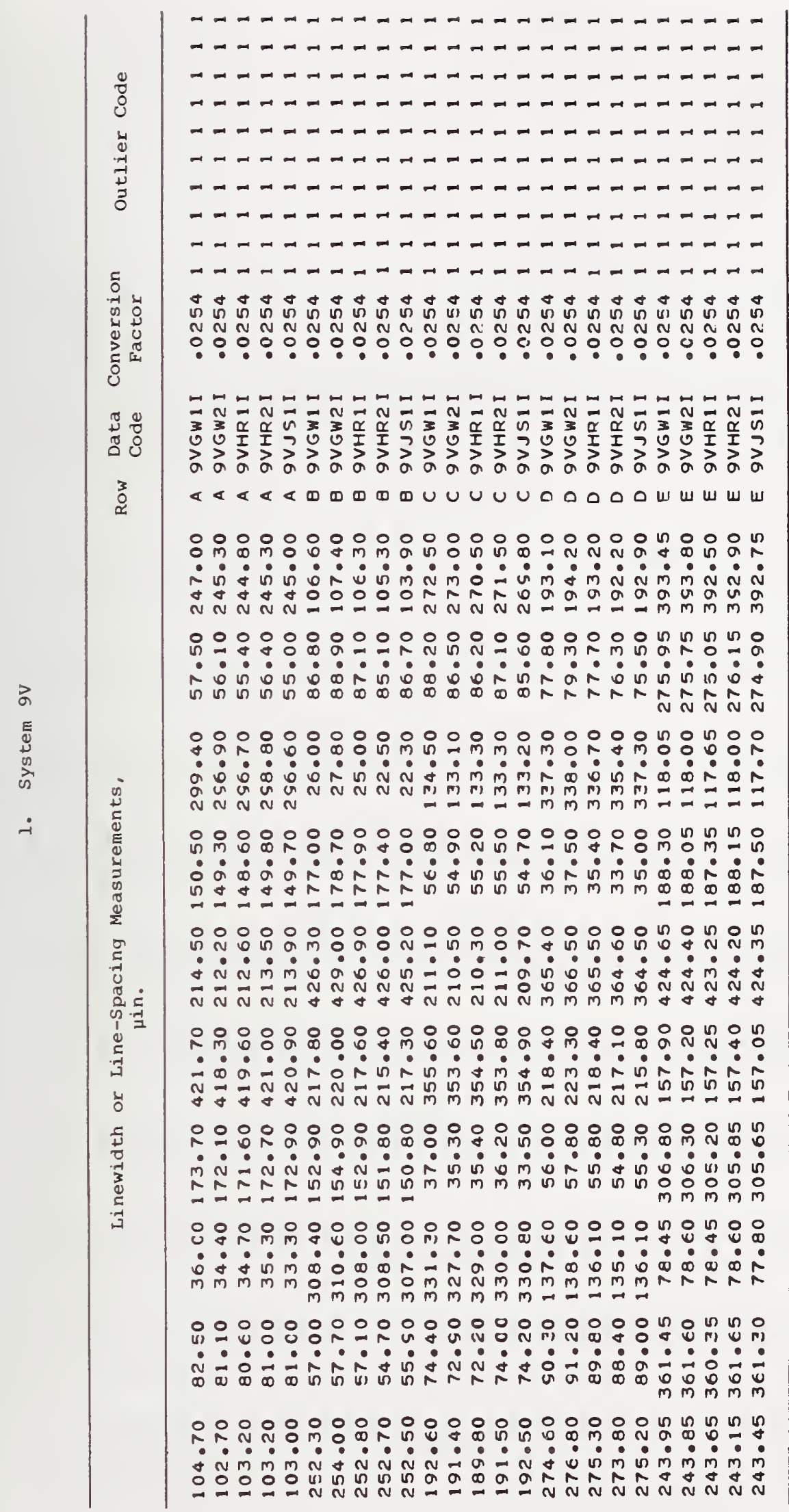

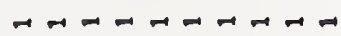

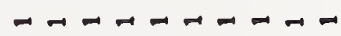

$\sim-a-a-a-a$

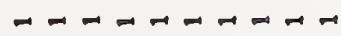

$\leadsto----\rightarrow-\mu-$

- - -નન-ન-ન

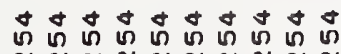

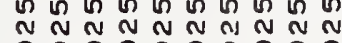

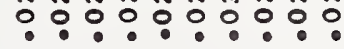

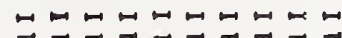
$\vec{\alpha} \overrightarrow{\underline{u}} \overrightarrow{\underline{\alpha}} \vec{u} \overrightarrow{\underline{\alpha}} \overrightarrow{\underline{\alpha}} \overrightarrow{\underline{u}} \overrightarrow{\underline{\alpha}} \vec{u}$

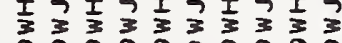

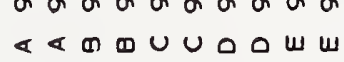

응ㅇㅇㅇㅇㅇㅇ은 $\dot{0} \dot{m} \dot{0} \dot{0} \dot{a} \dot{0} \dot{\sim} \dot{m}$ N

웅ㅇㅇㅇㅇㅇㅇㅇㅡ n

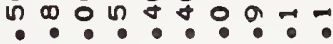
$\dot{0} \dot{0} \dot{0} \dot{0} \dot{0} \dot{0} \dot{\sim}$ in ถึก

- 00000000 in mㅇำ $\dot{\infty} \dot{0} \dot{0} \dot{m} \dot{0} \dot{0} \dot{\infty}$ N $N$ N

우앙웅응ㅇㅇㅇㅇㅛ 의

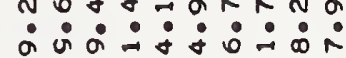
品

웅ㅇㅇㅇㅇㅢ

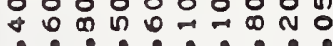
$\dot{N}=\dot{\sim} \dot{\infty} \dot{\infty} \dot{\infty} \dot{\sim} \dot{0}$

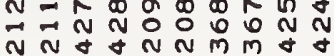

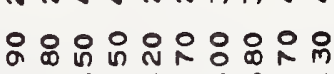

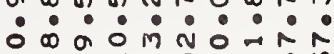

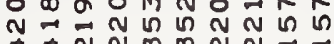
응ㅇㅇㅇㅇㅇㅇㅇㄴ은 ก:

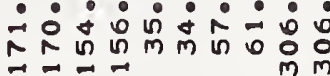

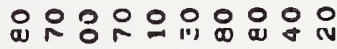
$\dot{0} \dot{0} \dot{0} \dot{0} \dot{0} \dot{0}$

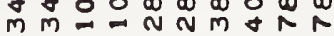
m $m$ m $m$

응ㅇㅇㅇㅇㅇㅇ oror $\dot{0} \dot{0} \dot{\sim} \dot{N} \dot{\sim} \dot{0} \dot{0}$ ○

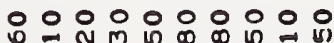
$\because \div: \div: \div$ N

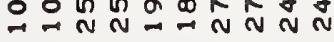




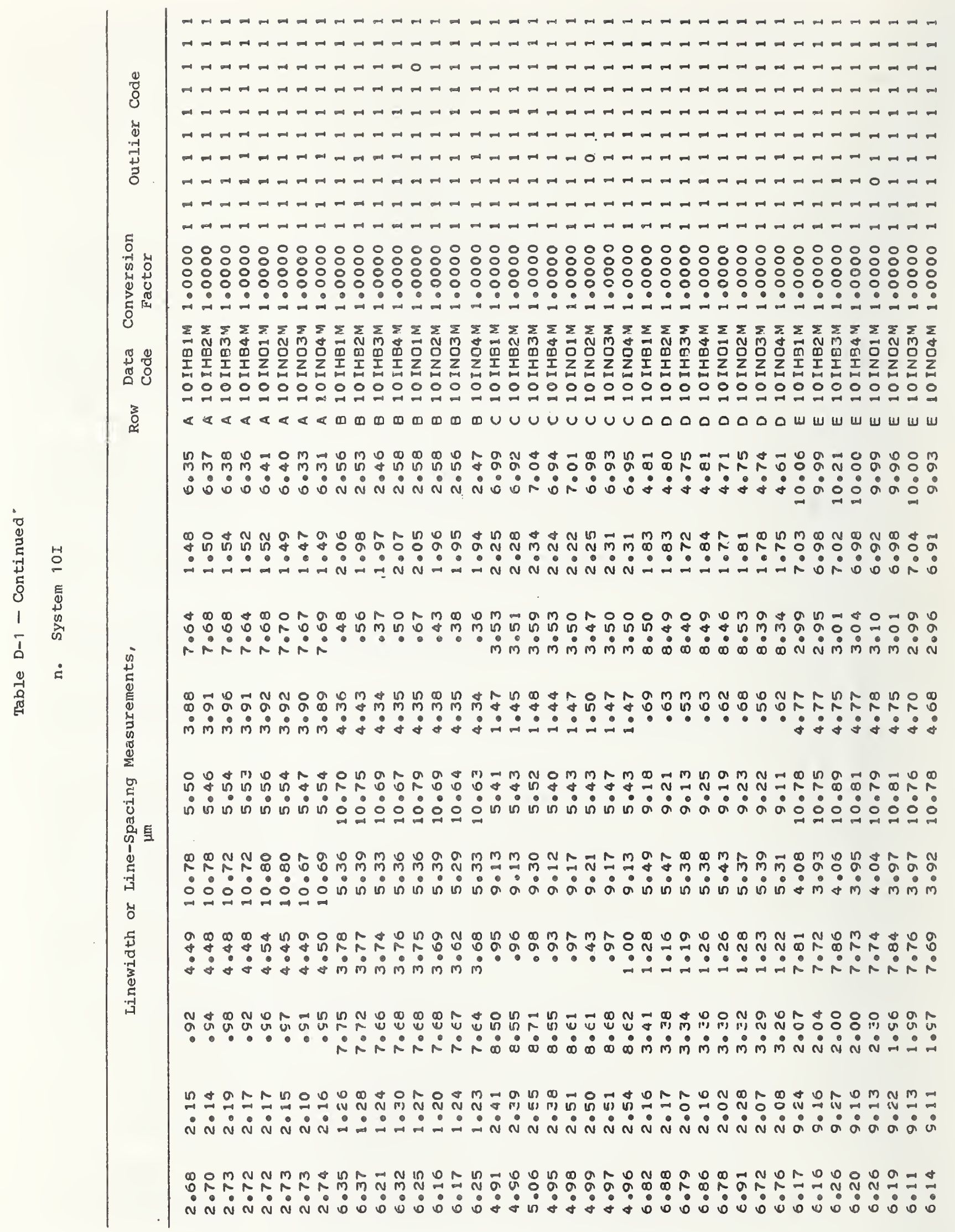




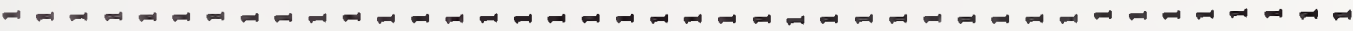

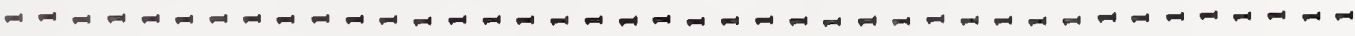

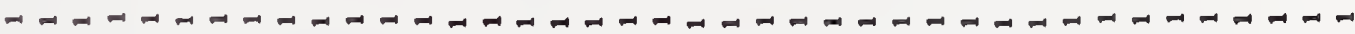

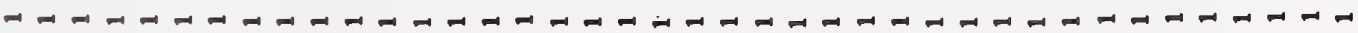

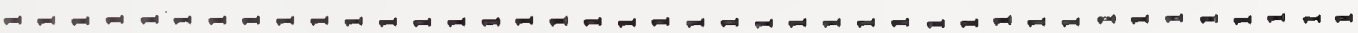

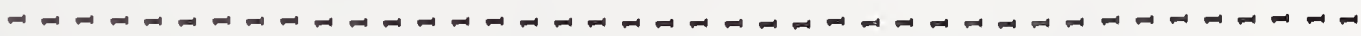

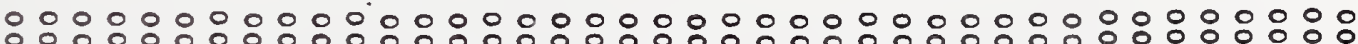

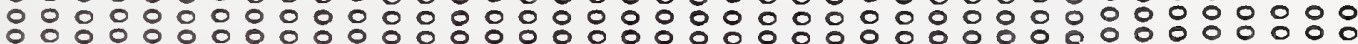

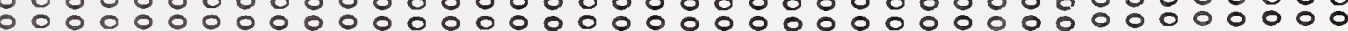

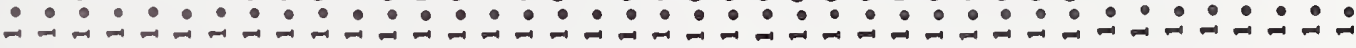

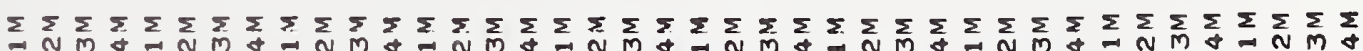

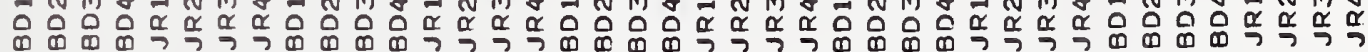

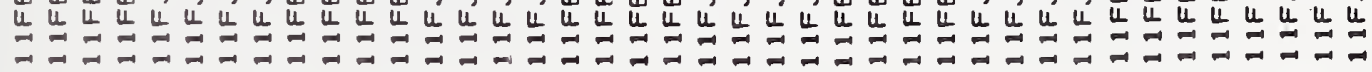
$<<<<<<<<\infty$ क \% ก

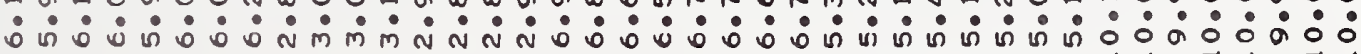

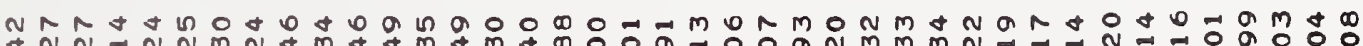

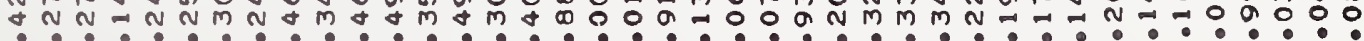

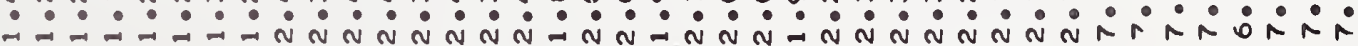

a a o n

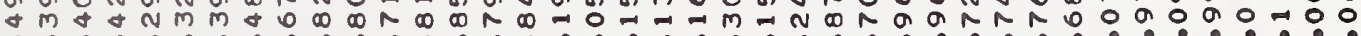

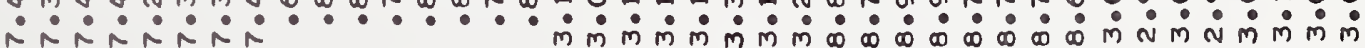

ก ก

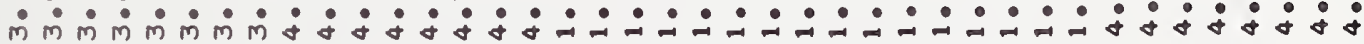

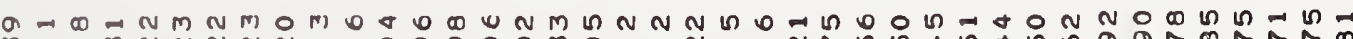

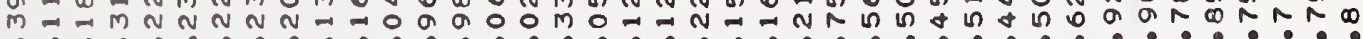

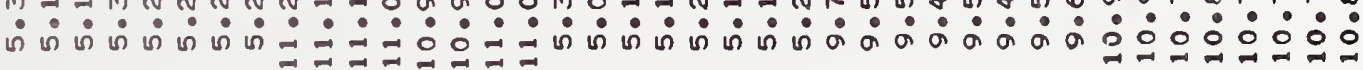

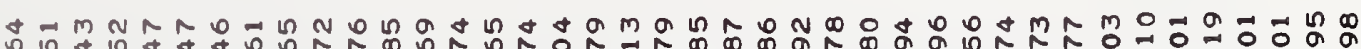
$\dot{0} \dot{0} \dot{0} \dot{0} \dot{0} \dot{0}$ i $\dot{0}$ ம

Nam

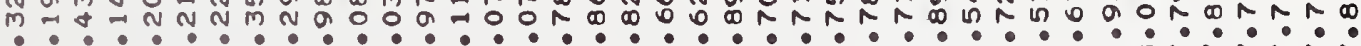

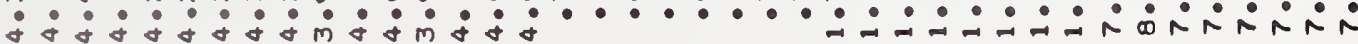

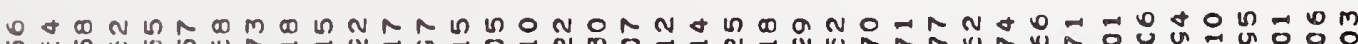
ถะแ - - - $\dot{\infty} \dot{\infty} \dot{\infty} \dot{\infty} \dot{\sim} \dot{\infty} \dot{\infty} \dot{\infty} \dot{\infty} \dot{\infty} \dot{\infty} \dot{\infty} \dot{\infty} \dot{\infty} \dot{\infty} \dot{m} \dot{m} \dot{m} \dot{m} \dot{m} \dot{m} \dot{m} \dot{N} \dot{N} \dot{N} \dot{N} \dot{N} \dot{N} \dot{N}$

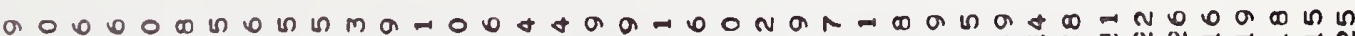
更

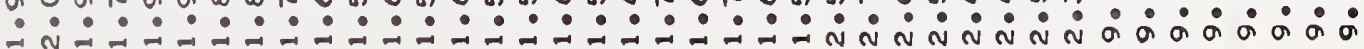

N

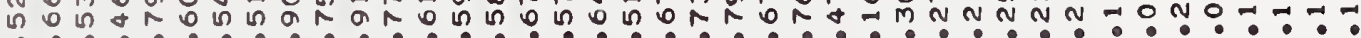

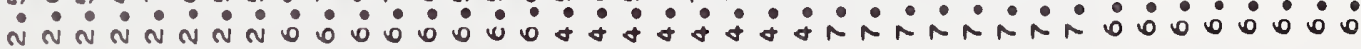




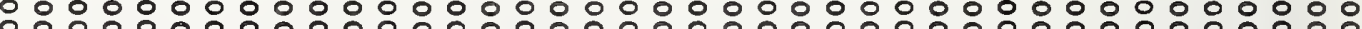

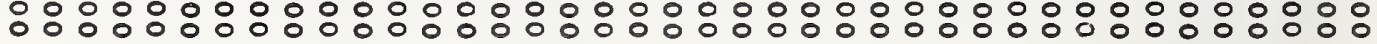

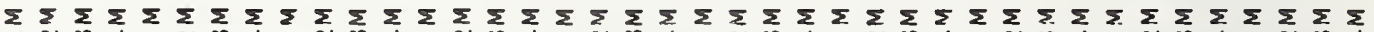

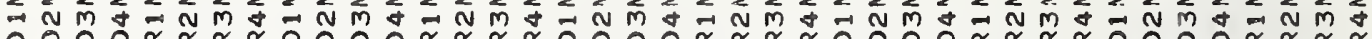

๘

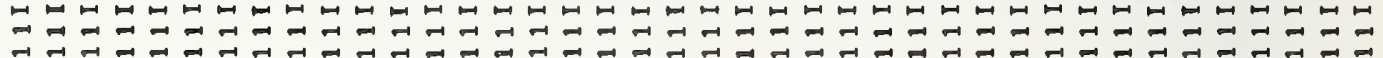

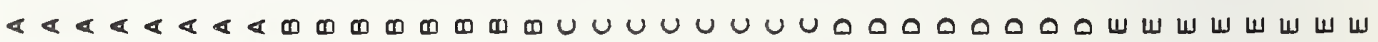

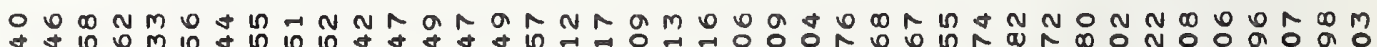
$\dot{0} \dot{0} \dot{0} \dot{0} \dot{0} \dot{N} \dot{N} \dot{N} \dot{N} \dot{N} \dot{N} \dot{\sim} \dot{\sim} \dot{\sim} \dot{\sim} \dot{\sim} \dot{j} \dot{+} \dot{+} \dot{+} \dot{+} \dot{+} \dot{0} \dot{0} \dot{0} \dot{0} \dot{0} \dot{0}$

구 궁ำ

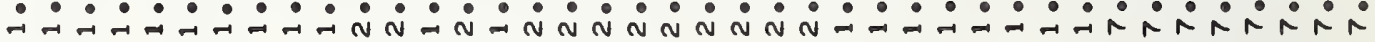

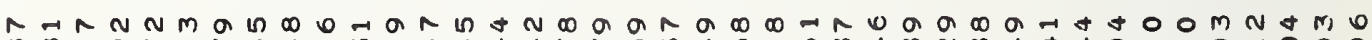
m d $\dot{i} \dot{i} \dot{i} \dot{R} \cdot \dot{m} \dot{m} \dot{m} \dot{m} \dot{m} \dot{m} \dot{m} \dot{\infty} \dot{\infty} \dot{\infty} \dot{\infty} \dot{\infty} \dot{\infty} \dot{\infty} \dot{\infty} \dot{\infty} \dot{m} \dot{m} \dot{m} \dot{m} \dot{m} \dot{m} \dot{m} \dot{m}$

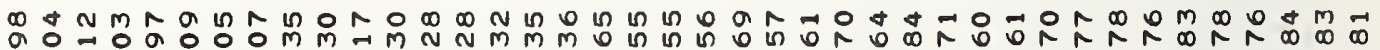

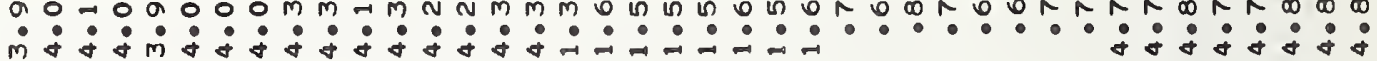

ค OND

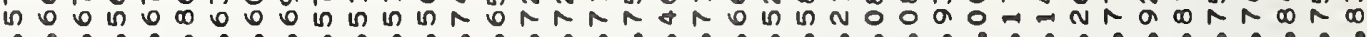
ம

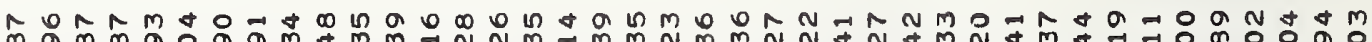

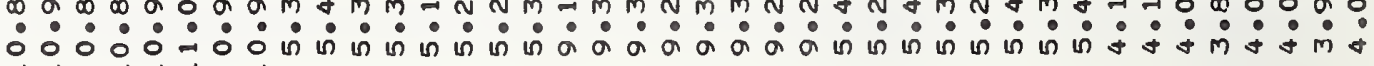

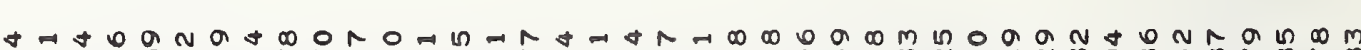
ถี่

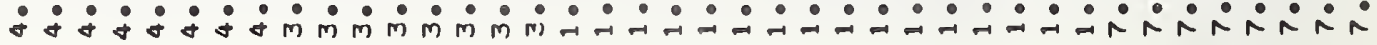

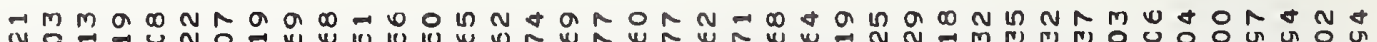

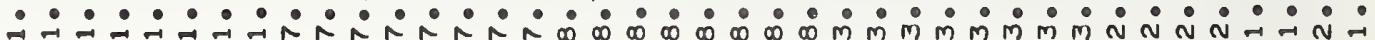

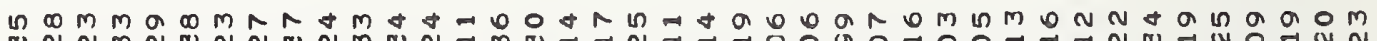

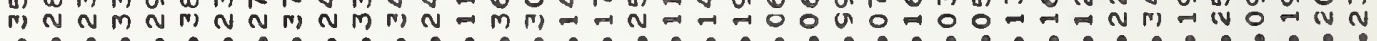

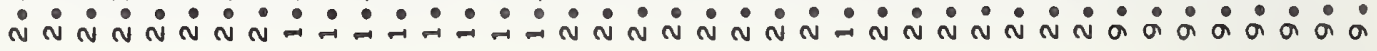

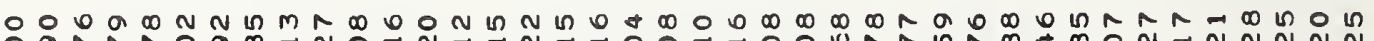
odk

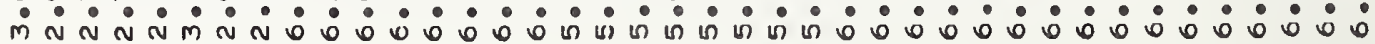


(

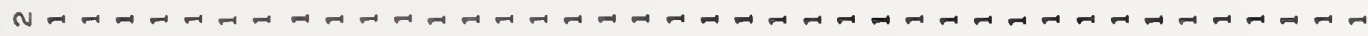

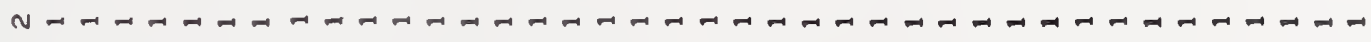

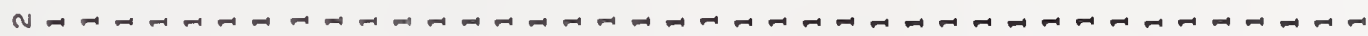

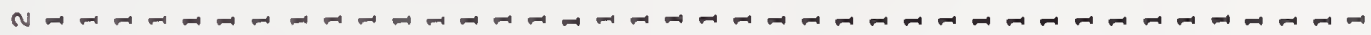

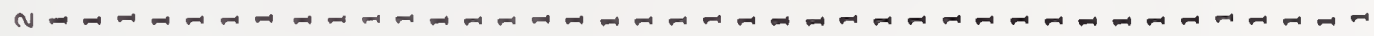

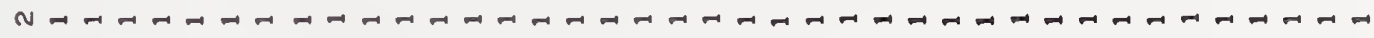

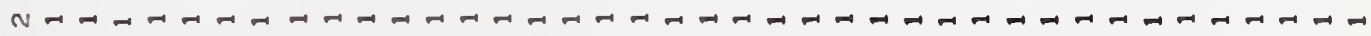

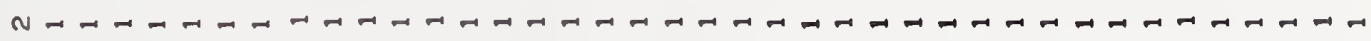

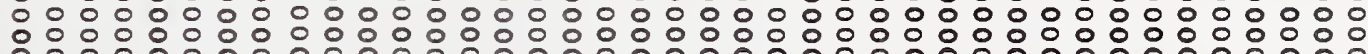
0000000000000000000000000000000000000000

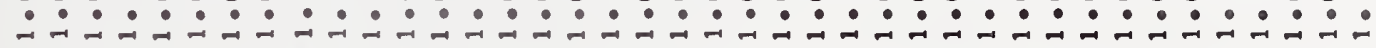

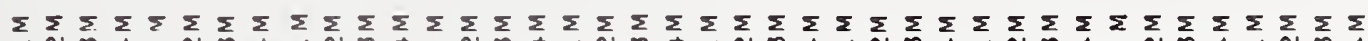

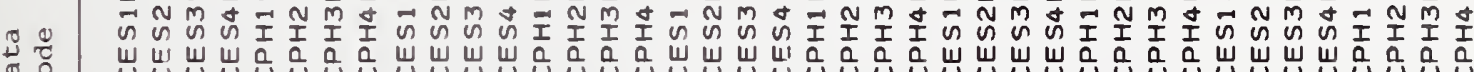

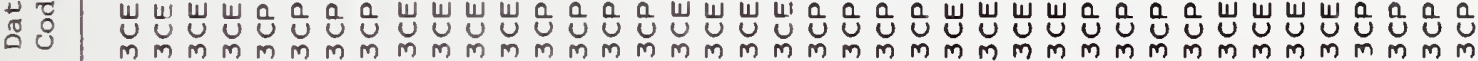
$m m m m m m m m m m m m m m m m m m m m m m m m m g m m m m m m m m m m m m m m$

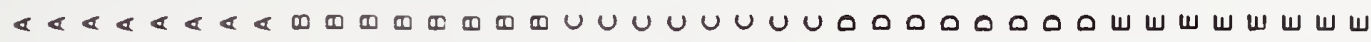

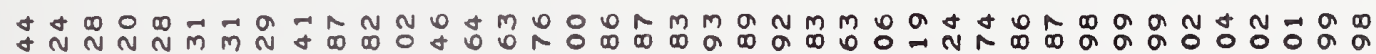
$\dot{0} \dot{0} \dot{0} \dot{0} \dot{0} \dot{N} \dot{N} \dot{N} \dot{N} \dot{N} \dot{N} \dot{0} \dot{0} \dot{0} \dot{0} \dot{0} \dot{0} \dot{0} \dot{0} \dot{\alpha} \dot{\alpha} \dot{\sigma} \dot{0} \dot{0} \dot{0} \dot{0} \dot{0}$

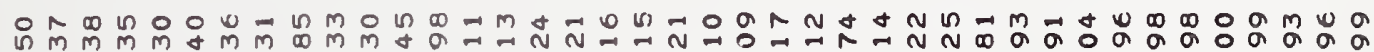

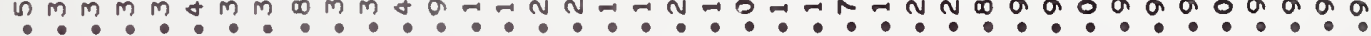

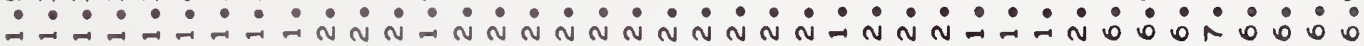

HD

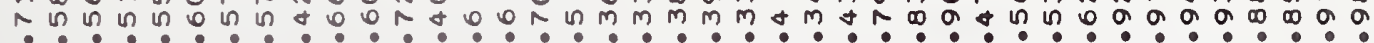

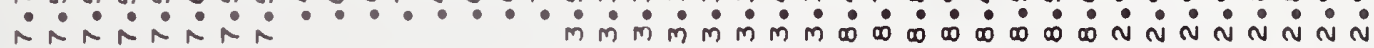

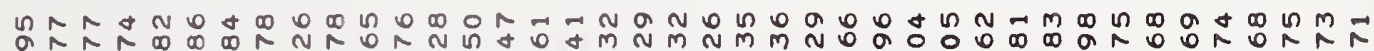

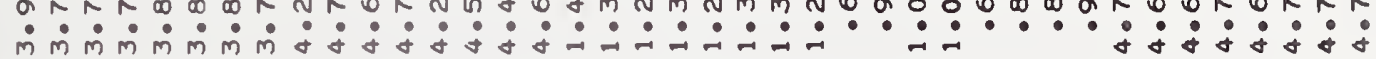

0 ○ 0 0

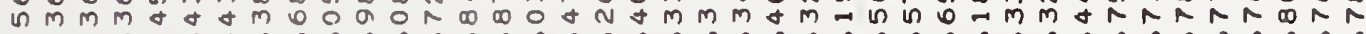

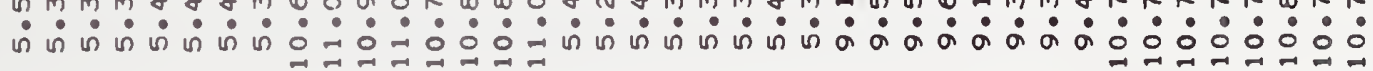

कิำ

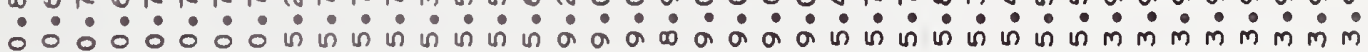
$\lim _{\rightarrow \rightarrow-1} \rightarrow-1$

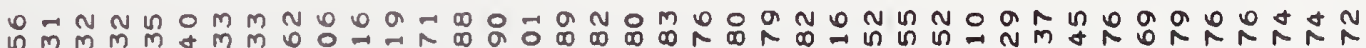

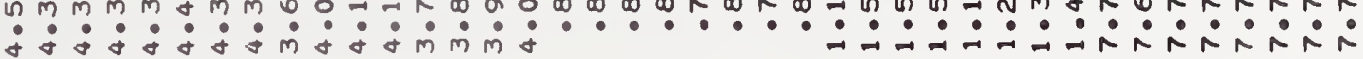

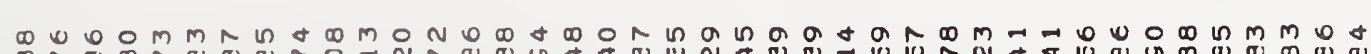

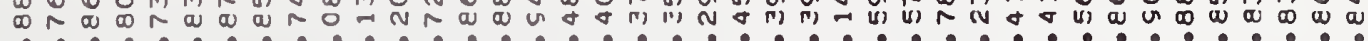
-

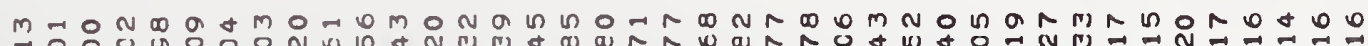

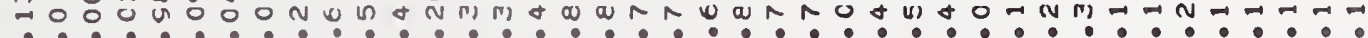

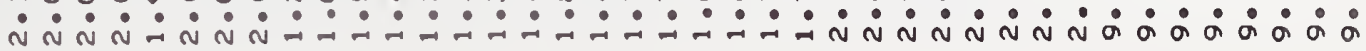

N ก! เ

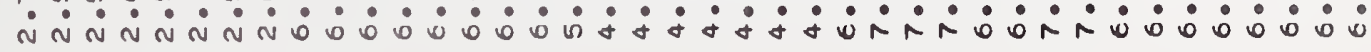




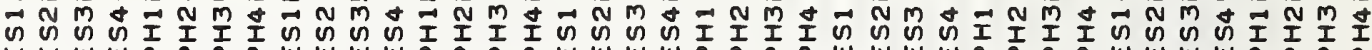

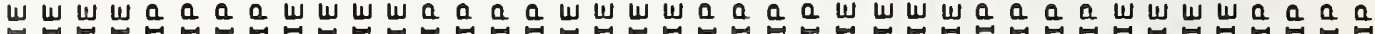

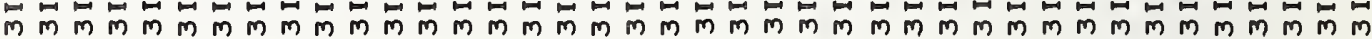

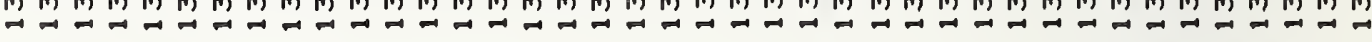

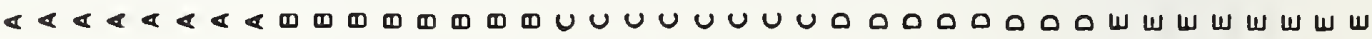
舟

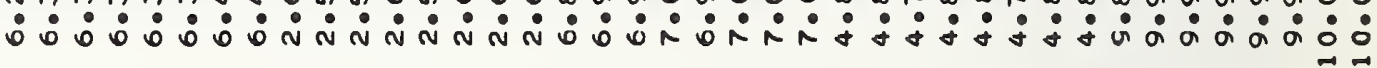

กต m.

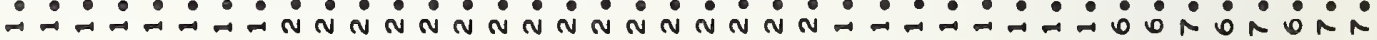

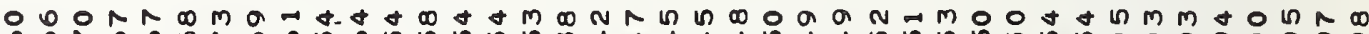
:

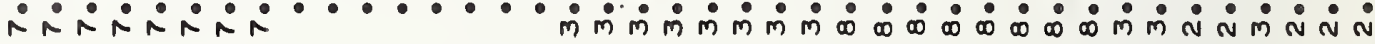

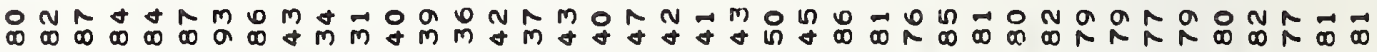

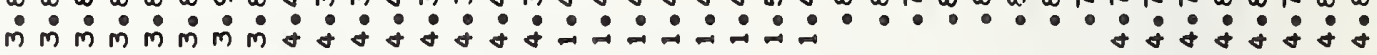

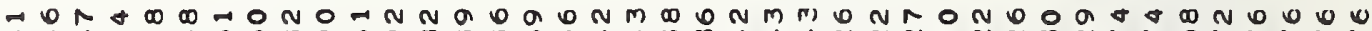
$+d+a t a n$ n ம

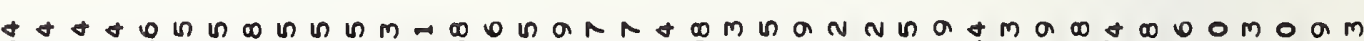

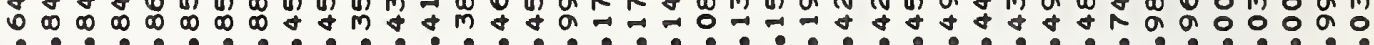

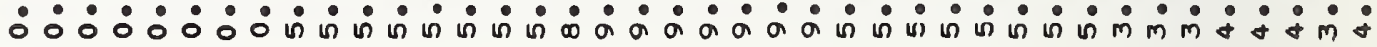

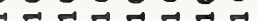

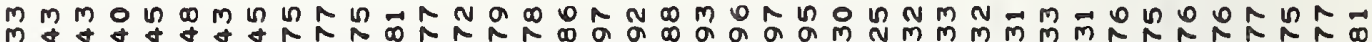

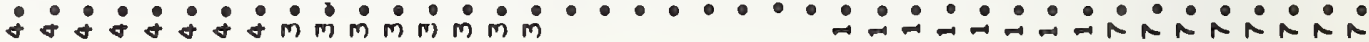

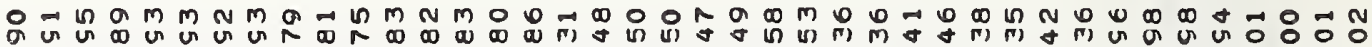

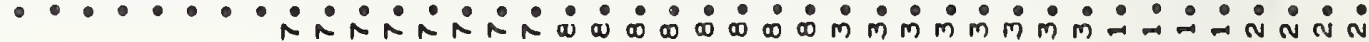

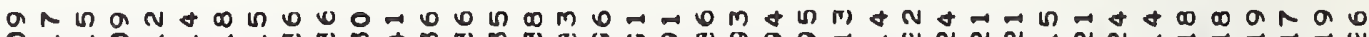

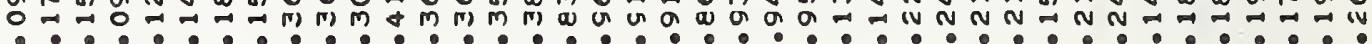

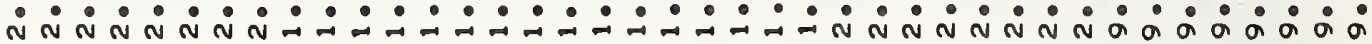

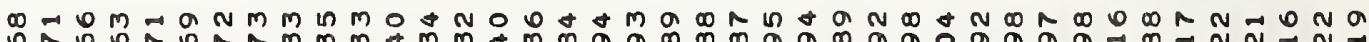
․․․

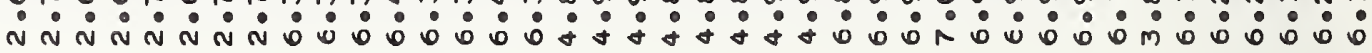


○ 0000000000000000000000000000000000000

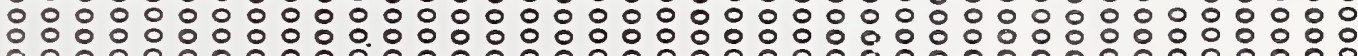
$\circ: 0: 00: 00: 00000000000000 \% 0000000000000000$

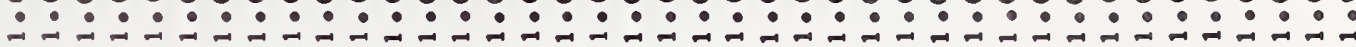
$\sum \sum \sum \sum \sum \sum \sum \sum \sum \sum \sum \sum \sum \sum \sum \sum \sum \sum \sum \sum \sum \sum \sum \sum \sum \sum \sum \sum \sum \sum \sum \sum \sum \sum \sum \sum \sum \sum \sum \sum$

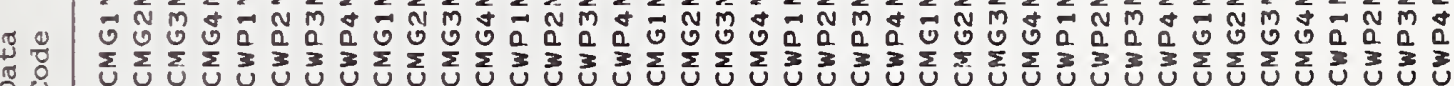
更 0

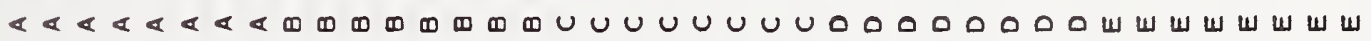

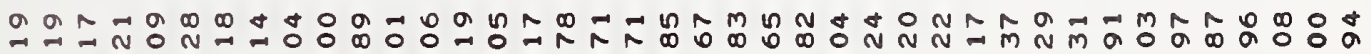

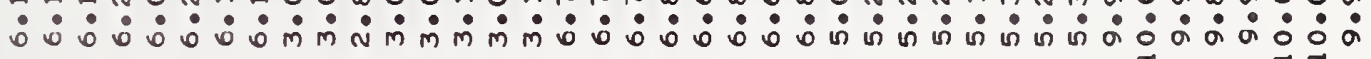

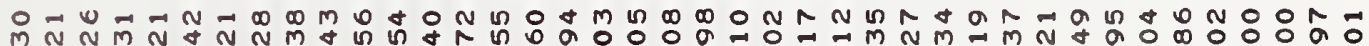

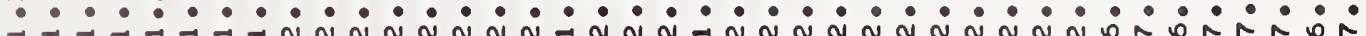

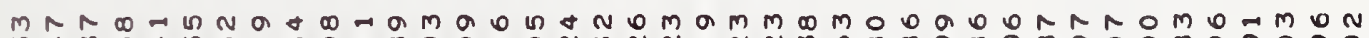

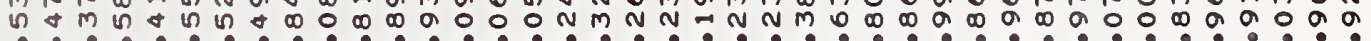
$\dot{i} \dot{i} \dot{i} \dot{i} \dot{i} \cdot \dot{-} \dot{\sim} \dot{m} \dot{m} \dot{m} \dot{m} \dot{m} \dot{m} \dot{m} \dot{\infty} \dot{\infty} \dot{\infty} \dot{\infty} \dot{\infty} \dot{\infty} \dot{m} \dot{m} \dot{N} \dot{N} \dot{N} \dot{N} \dot{N}$

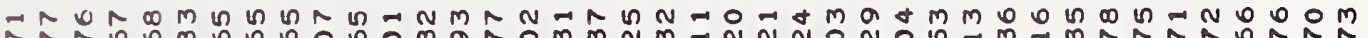

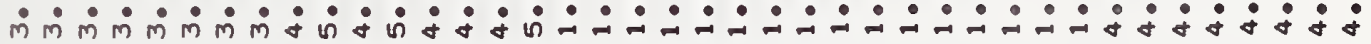

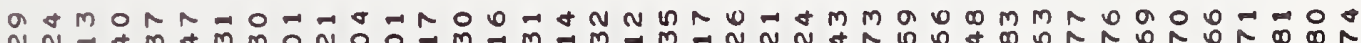

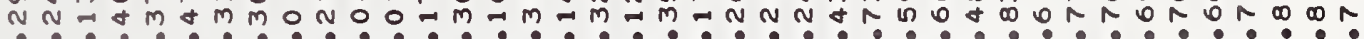
至 ம

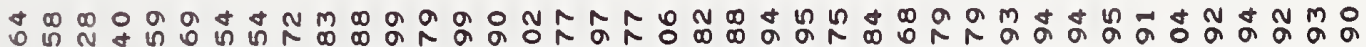

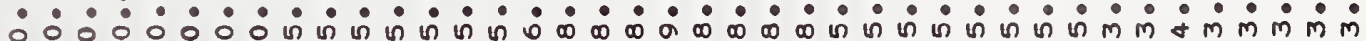

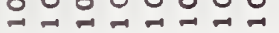

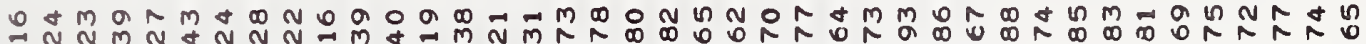

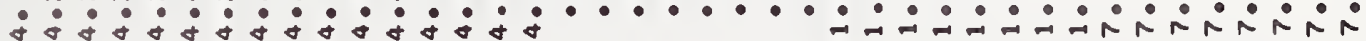

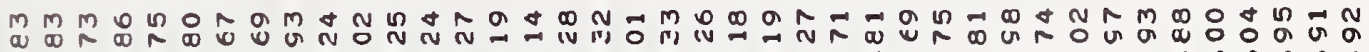

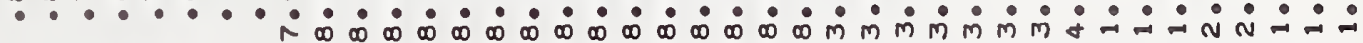

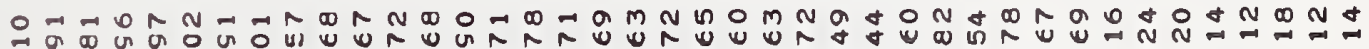

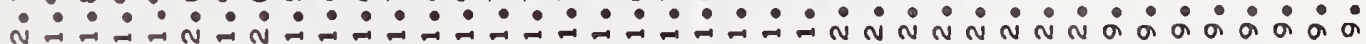

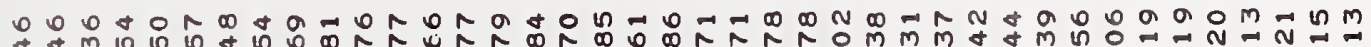

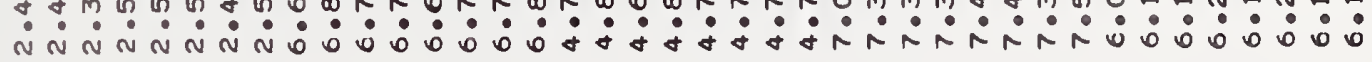




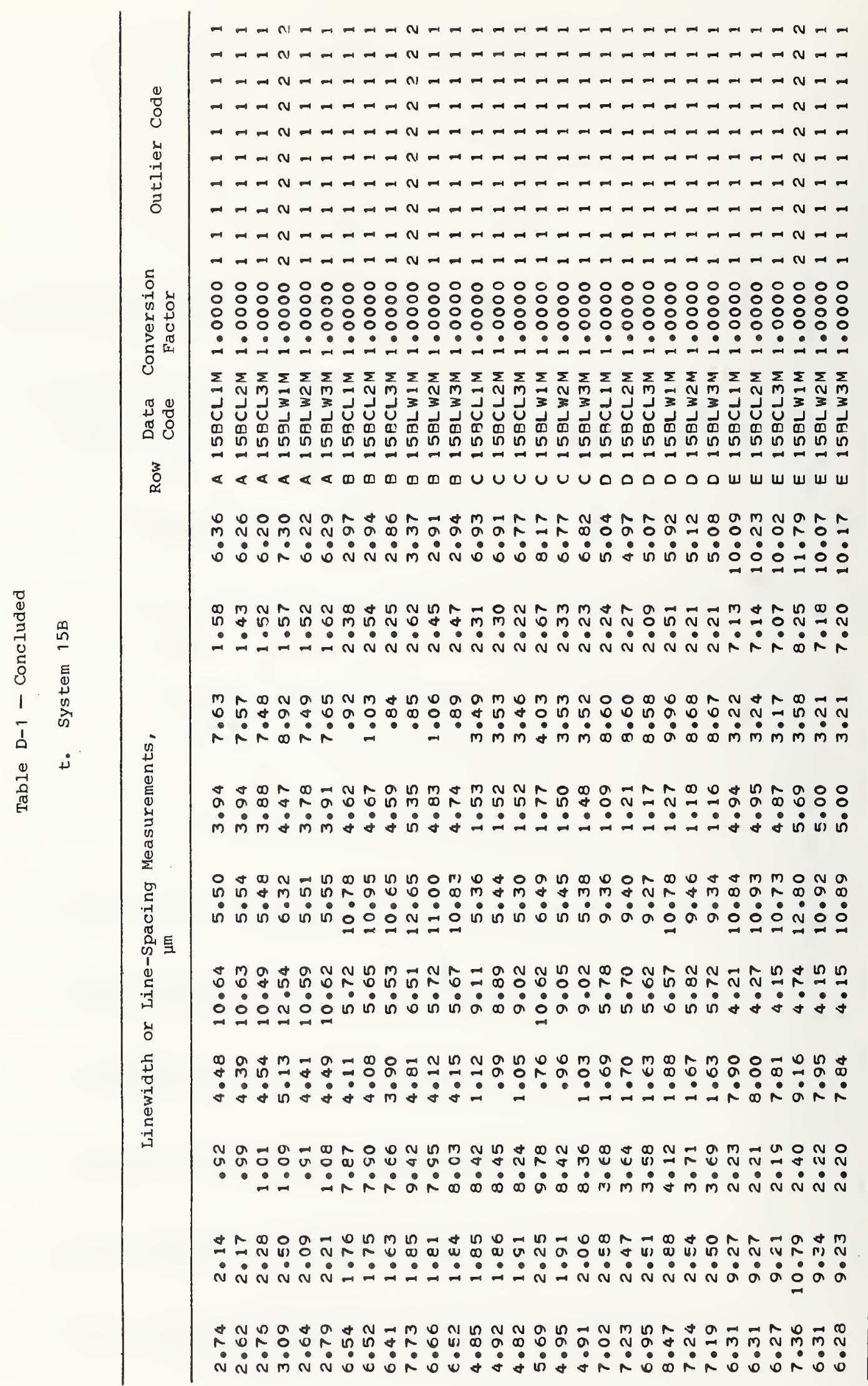




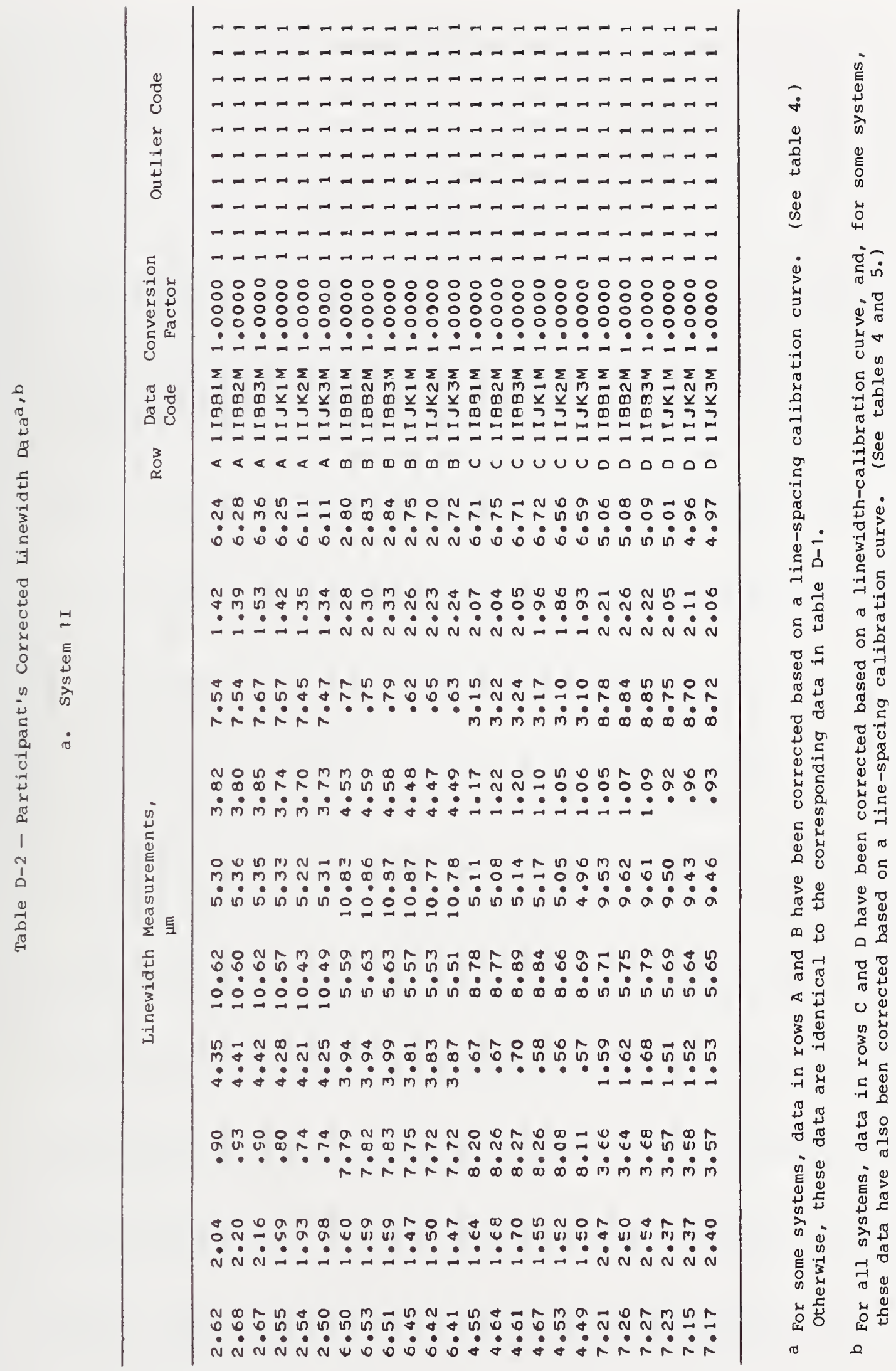




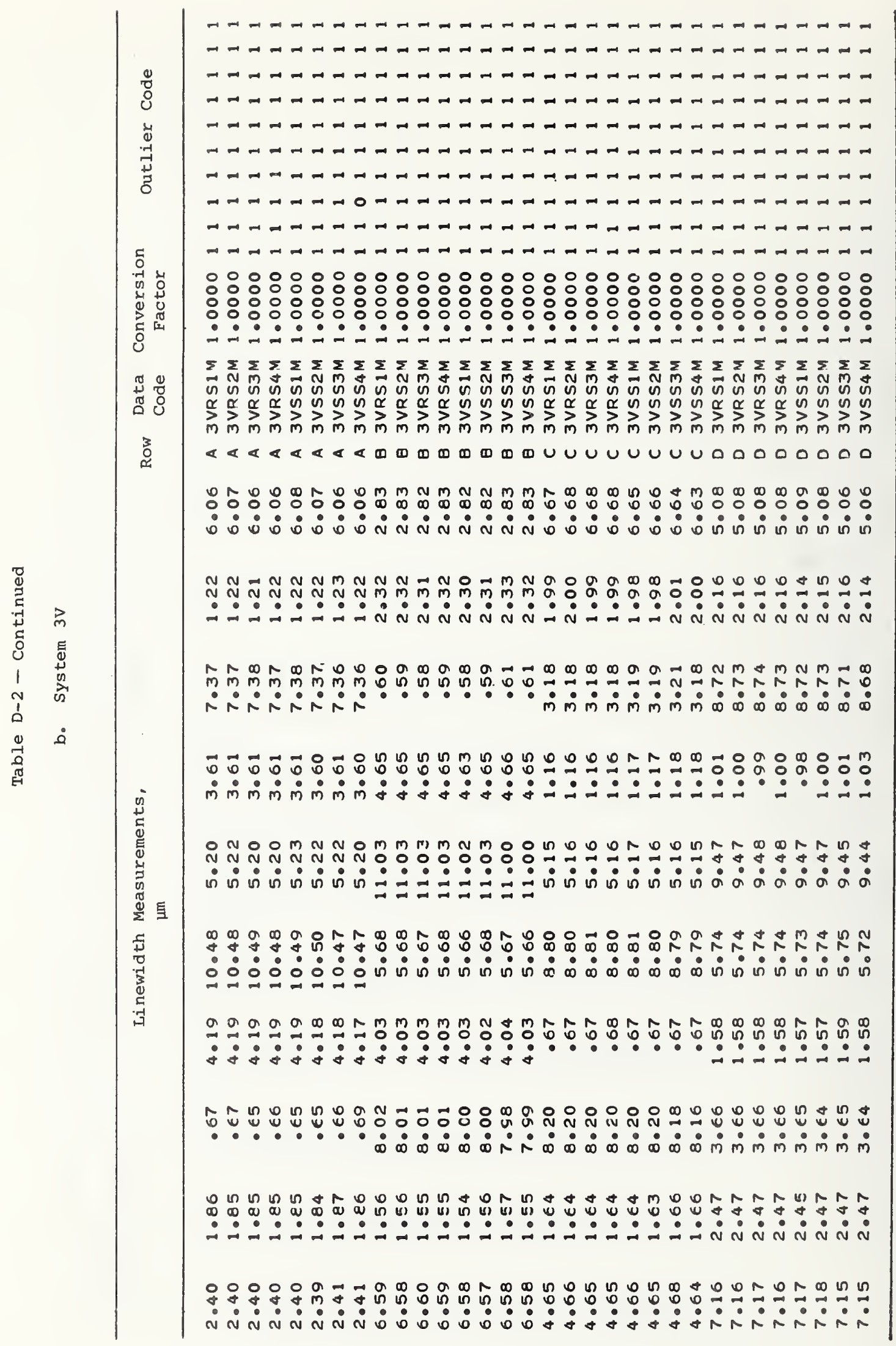


$$
-\frac{1}{20}
$$

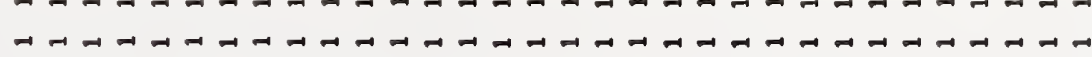

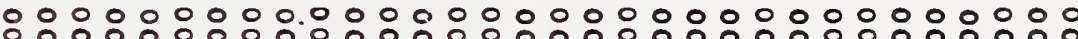

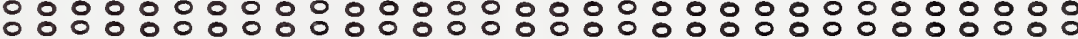

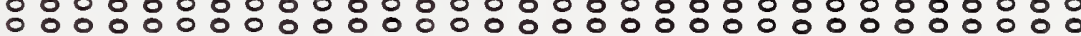

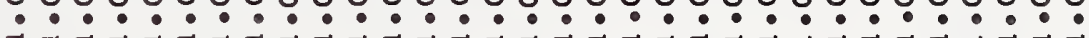
$\sum \sum \sum \sum \sum \sum \sum \sum \sum \sum \sum \sum \sum \sum \sum \sum \sum \sum \sum \sum \sum \sum \sum \sum \sum \sum \sum \sum \sum \sum \sum \sum$

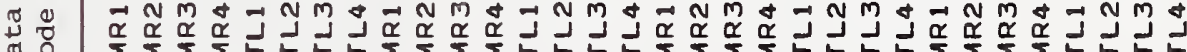

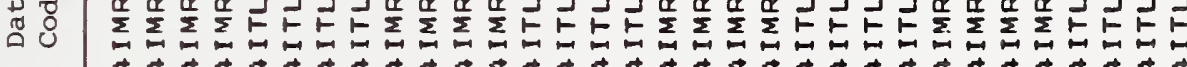

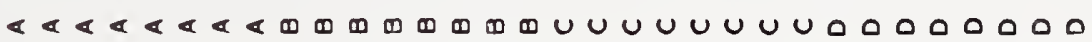

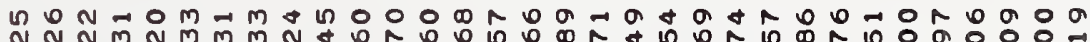

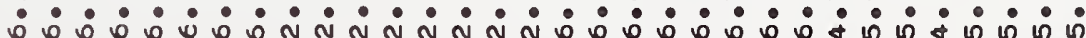

oก

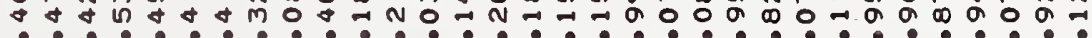

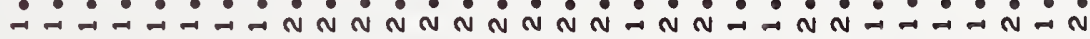

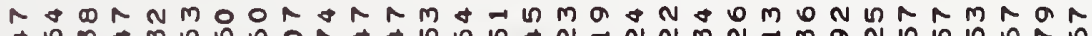

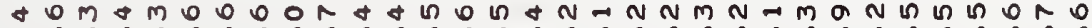
$\dot{i} \dot{i} \dot{i} \dot{i} \dot{a} \cdots \dot{m} \dot{m} \dot{m} \dot{m} \dot{m} \dot{m} \dot{m} \dot{\infty} \dot{\infty} \dot{\infty} \dot{\infty} \dot{\infty} \dot{\infty} \dot{\infty}$

+

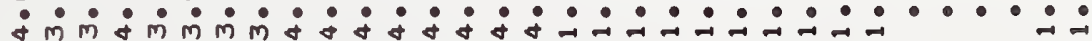

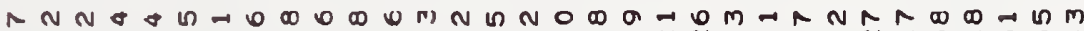

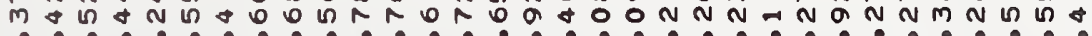
ம ம

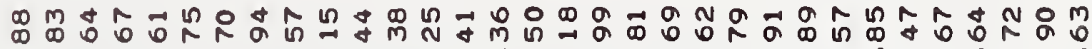

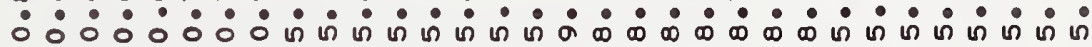
$\rightarrow \rightarrow-\rightarrow \rightarrow-1$

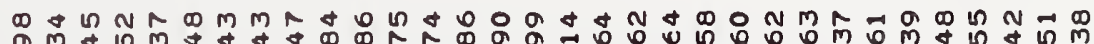

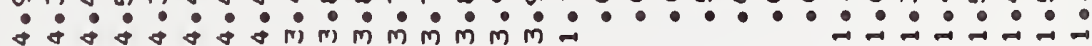

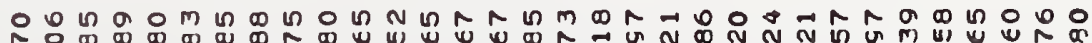
ก

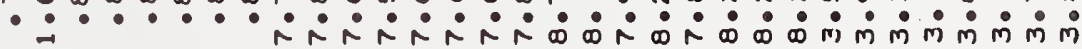

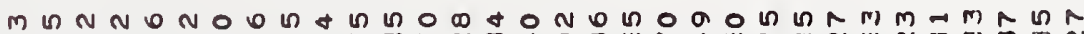

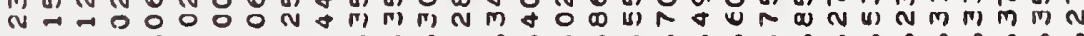

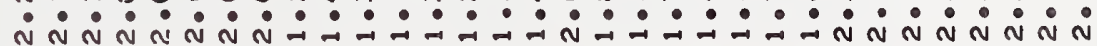

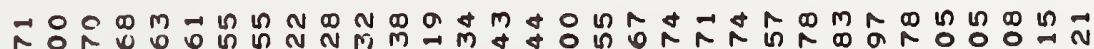

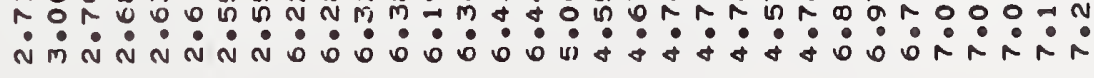




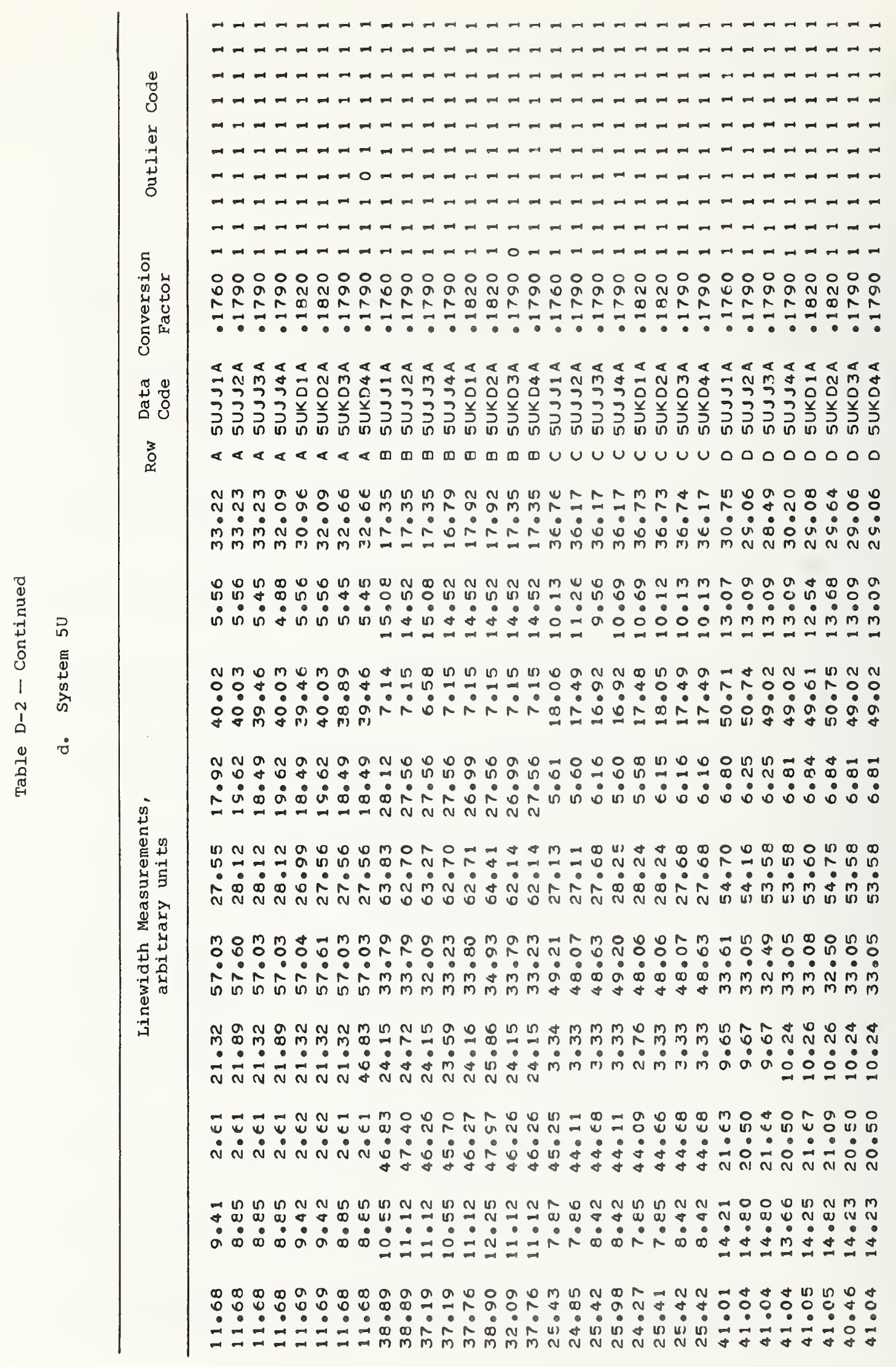




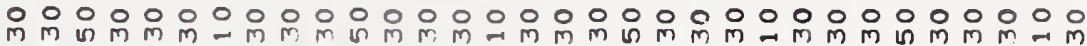

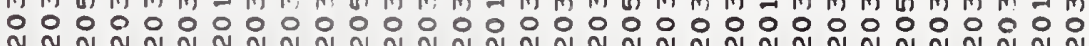

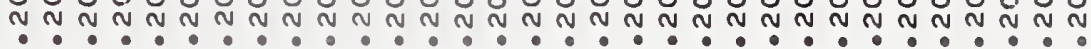

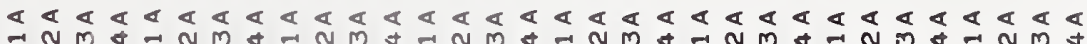

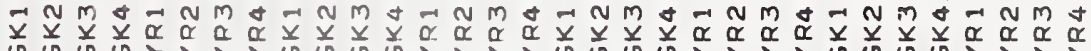

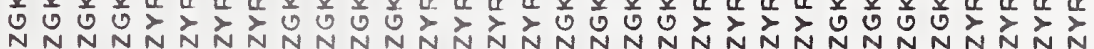

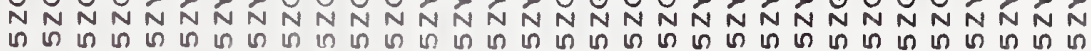
$\stackrel{3}{2}$

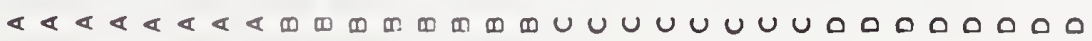

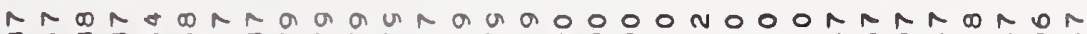

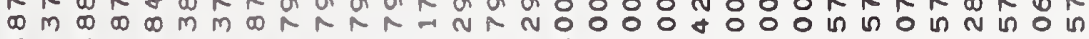

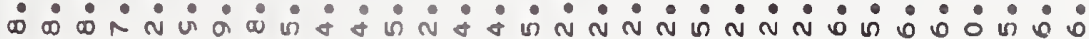

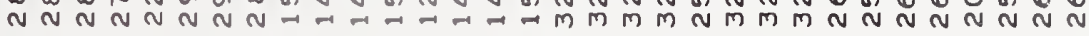

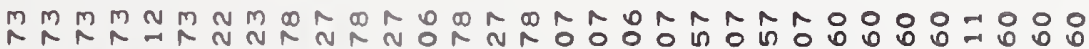

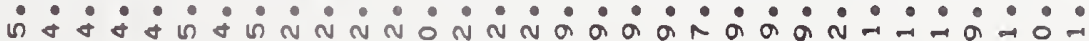
$\rightarrow \rightarrow-x_{-1} \rightarrow=$

$\Rightarrow F N A-H M M M M N M M M n$ n

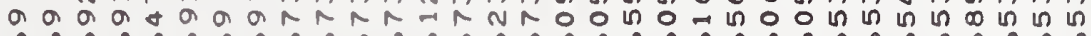

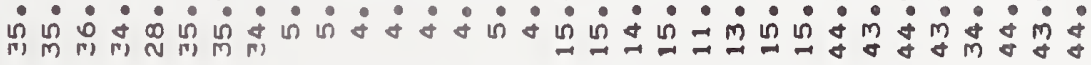

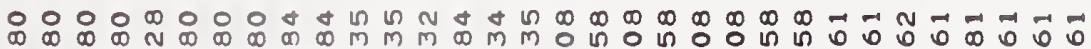

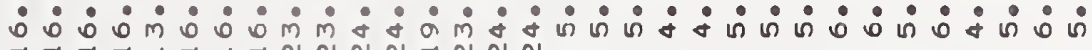

(4) แ) (ท)

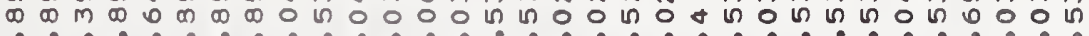

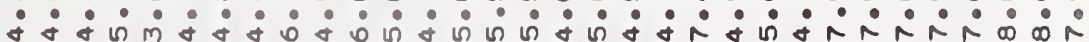

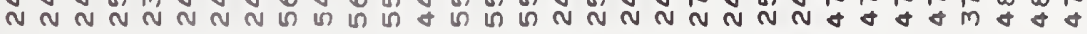

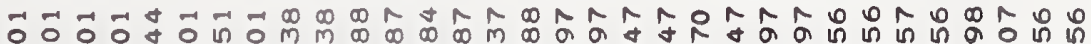

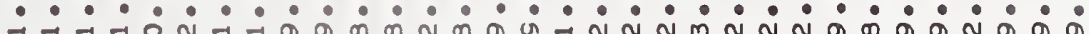
$\vec{b} \overrightarrow{0}$ in



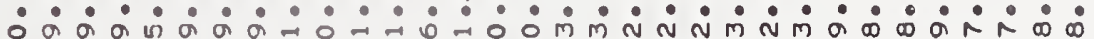

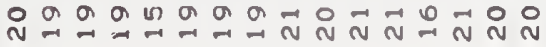

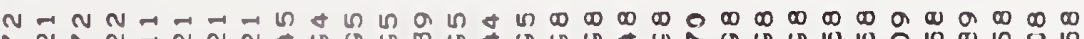
natenta

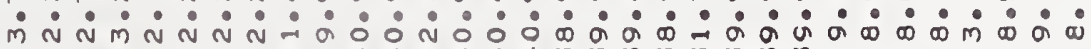

ถก ก ก ก

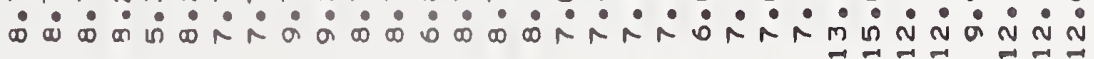

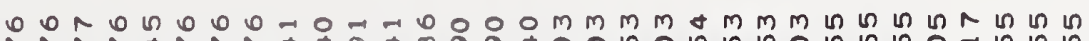
t.5.

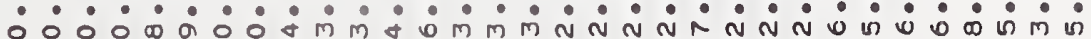

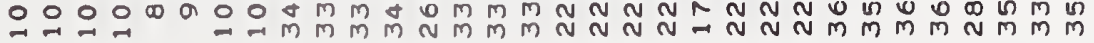




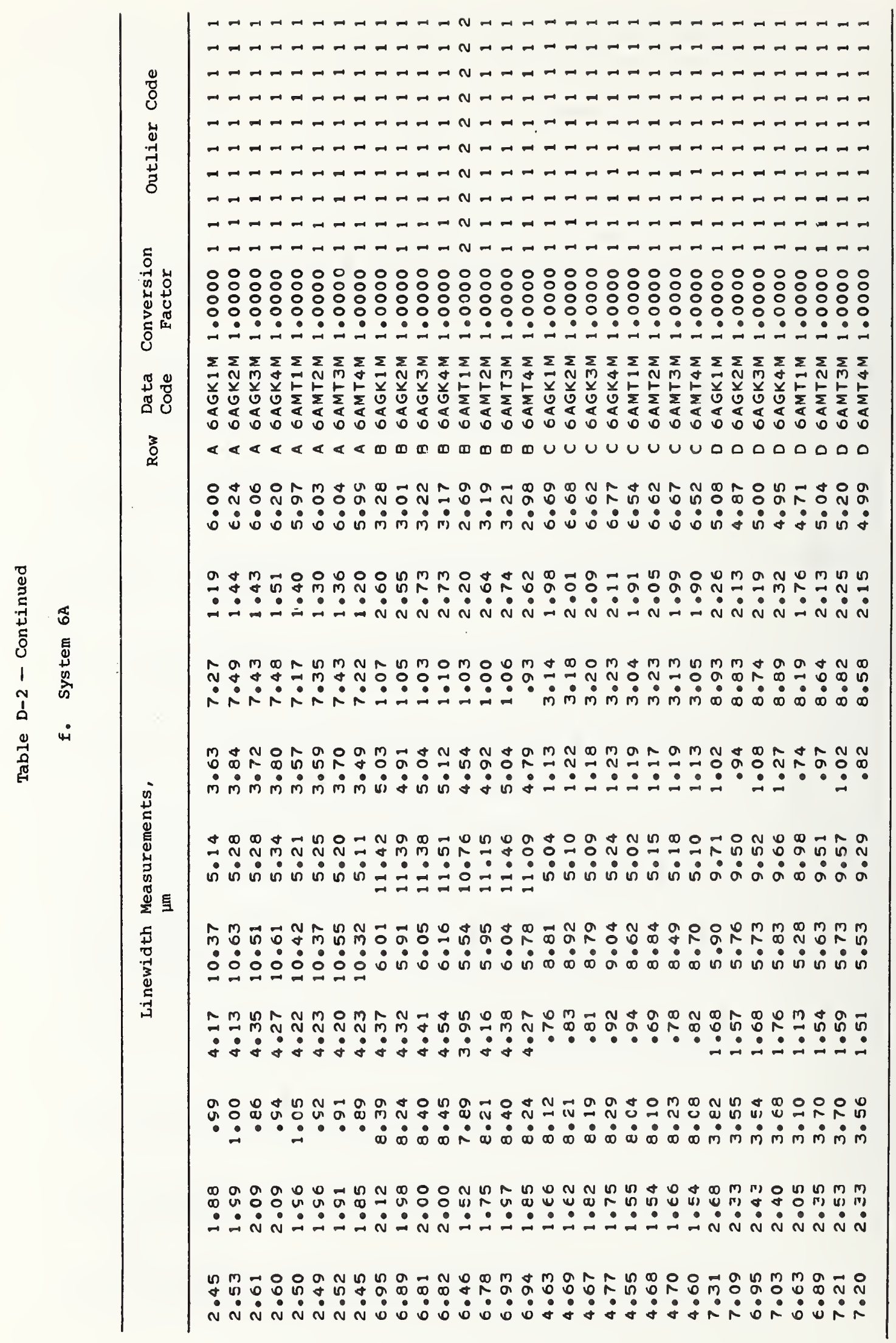




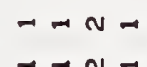

응ㅇㅇㅇㅇㅇㅇㅇㅇㅇㅇㅇㅇㅇㅇㅇㅇㅇㅇㅇㅇㅇㅇㅇㅇㅇㅇㅇㅇㅇㅇㅇㅇ응

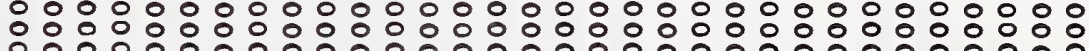
00000000000000000000000000000000

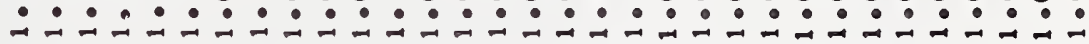

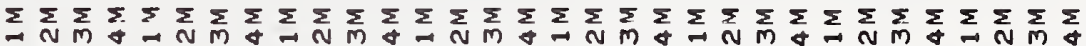
ב

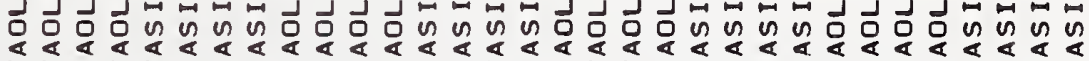

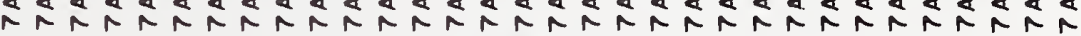

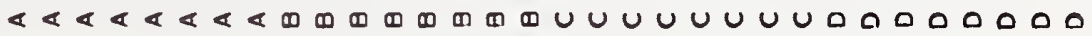

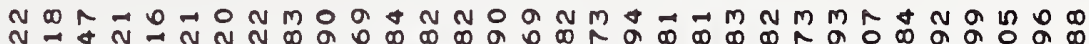
$\dot{0} \dot{0} \dot{0} \dot{0} \dot{0} \dot{0} \dot{N} \dot{N} \dot{N} \dot{N} \dot{N} \dot{N} \dot{N} \dot{0} \dot{0} \dot{0} \dot{0} \dot{0} \dot{0} \dot{0} \dot{0} \dot{q} \dot{q} \dot{\sigma} \dot{\sigma} \dot{q}$

$0 \div m \infty N n m a m$ n \& \&

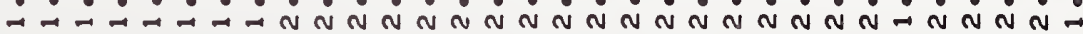

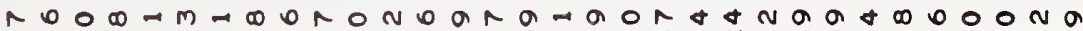
ถ⿻ㅇำ írírír

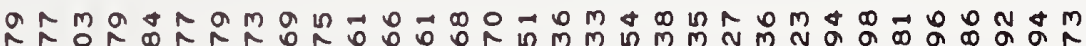
ํ.?? ? ? ? ? ? ?

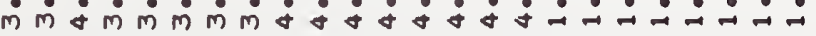

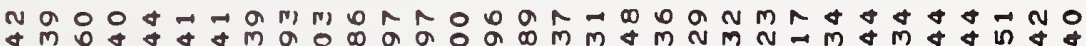
+mof ம

$\overrightarrow{0}$ 웅

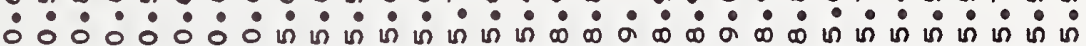
$\rightarrow-100 \div \div 0$

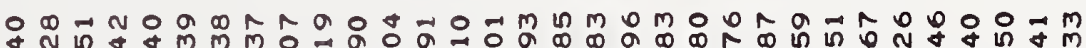

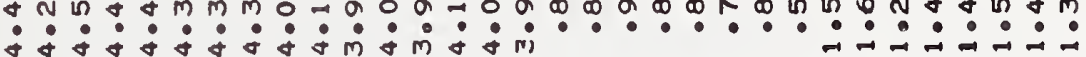

Oก 2 maGก

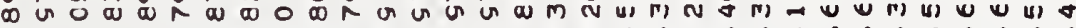

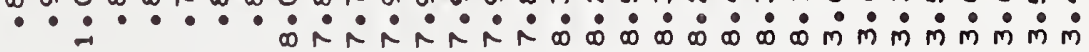

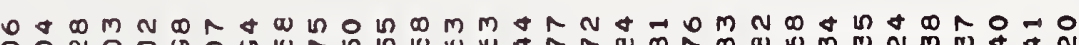

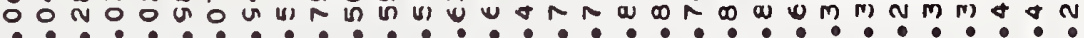

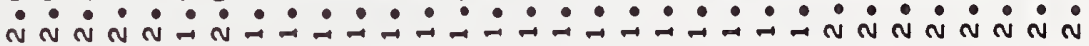

$m \infty \infty \vec{m} \sim \infty m \infty+n \vec{m} n a \infty \Rightarrow 0 m \infty \infty a+m a m 00 \infty \infty a \infty a$ ० 0 个 0 .

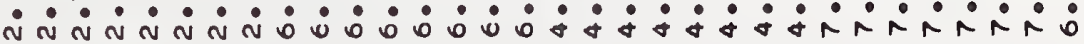




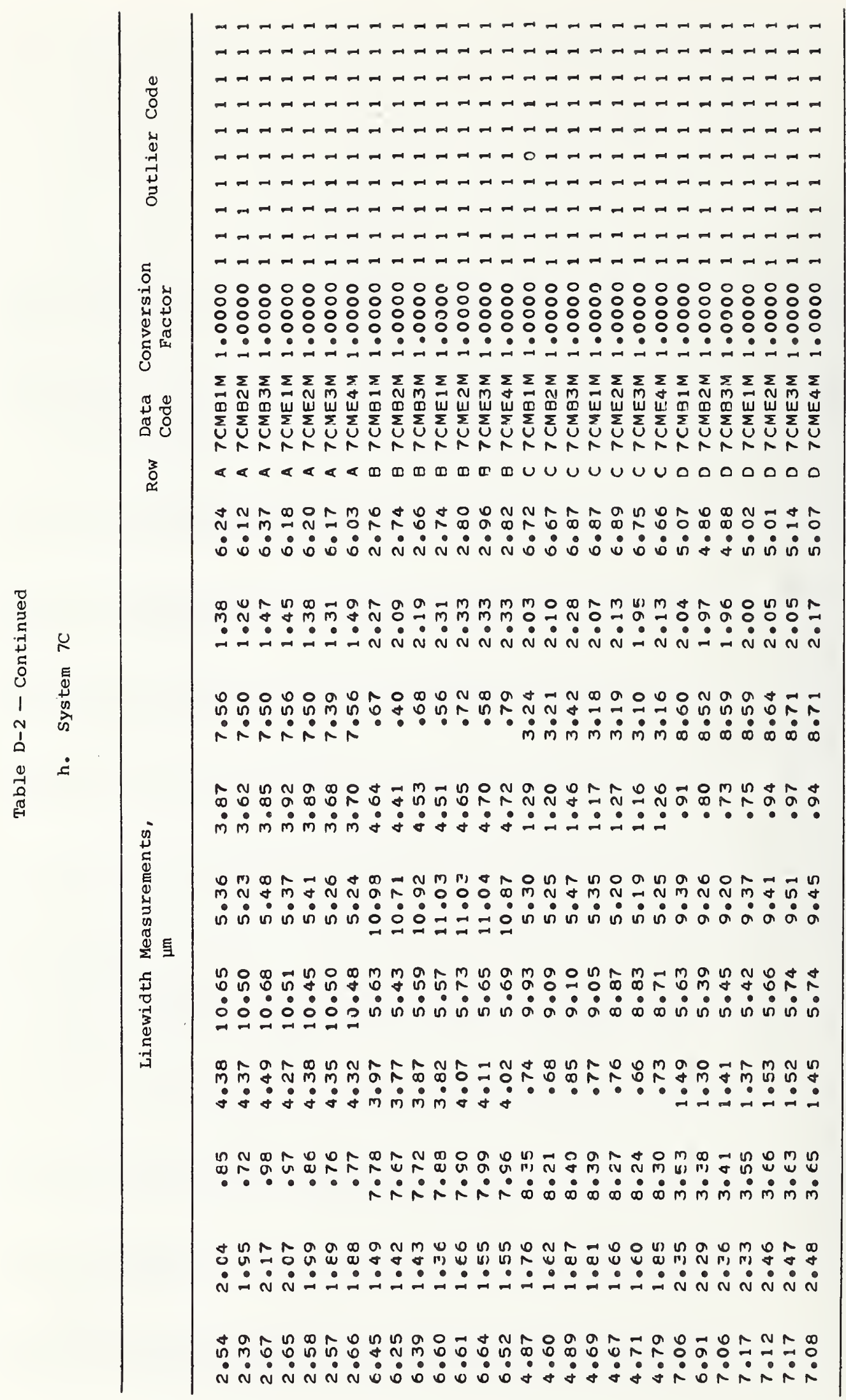




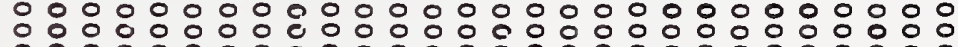
0000000000000000000000000000

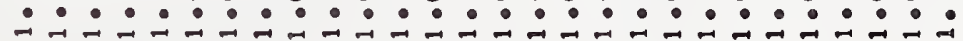

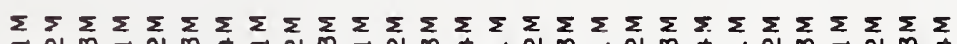

Ð

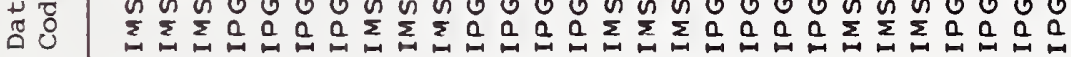

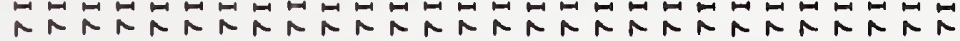

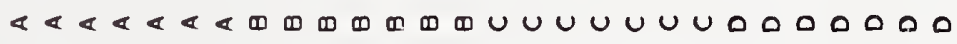

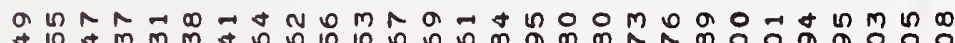
+ ก

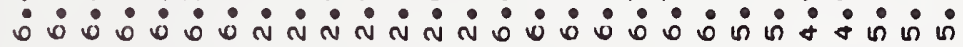

ำ

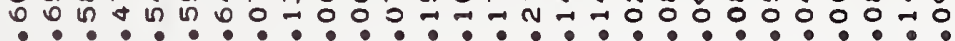
$\because-\dot{i}-\dot{i} \dot{N} \dot{N} \dot{N} \dot{N} \dot{N} \dot{N} \dot{N} \dot{N} \dot{N} \dot{N} \dot{N} \dot{N} \dot{N} \dot{N} \dot{N} \dot{N} \dot{N}$

nก ก inirini

n\&

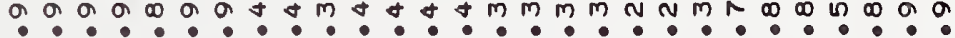

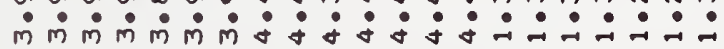

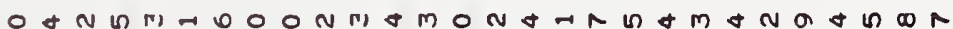
융

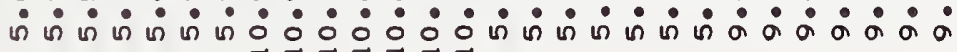

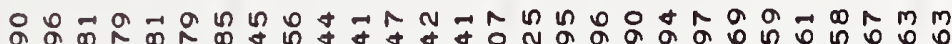
a

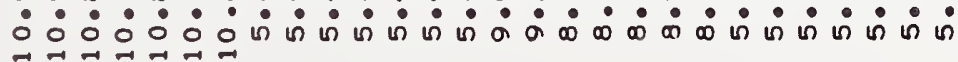

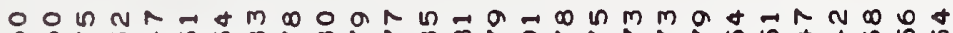
?

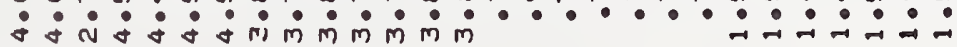

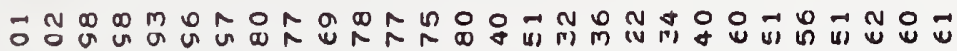
-

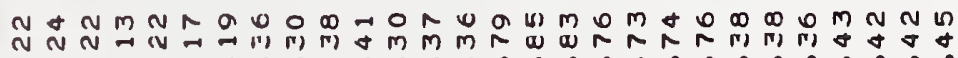

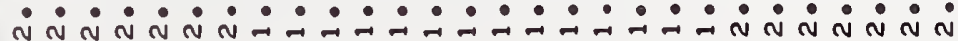

ค T R

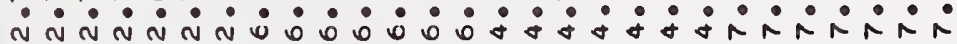




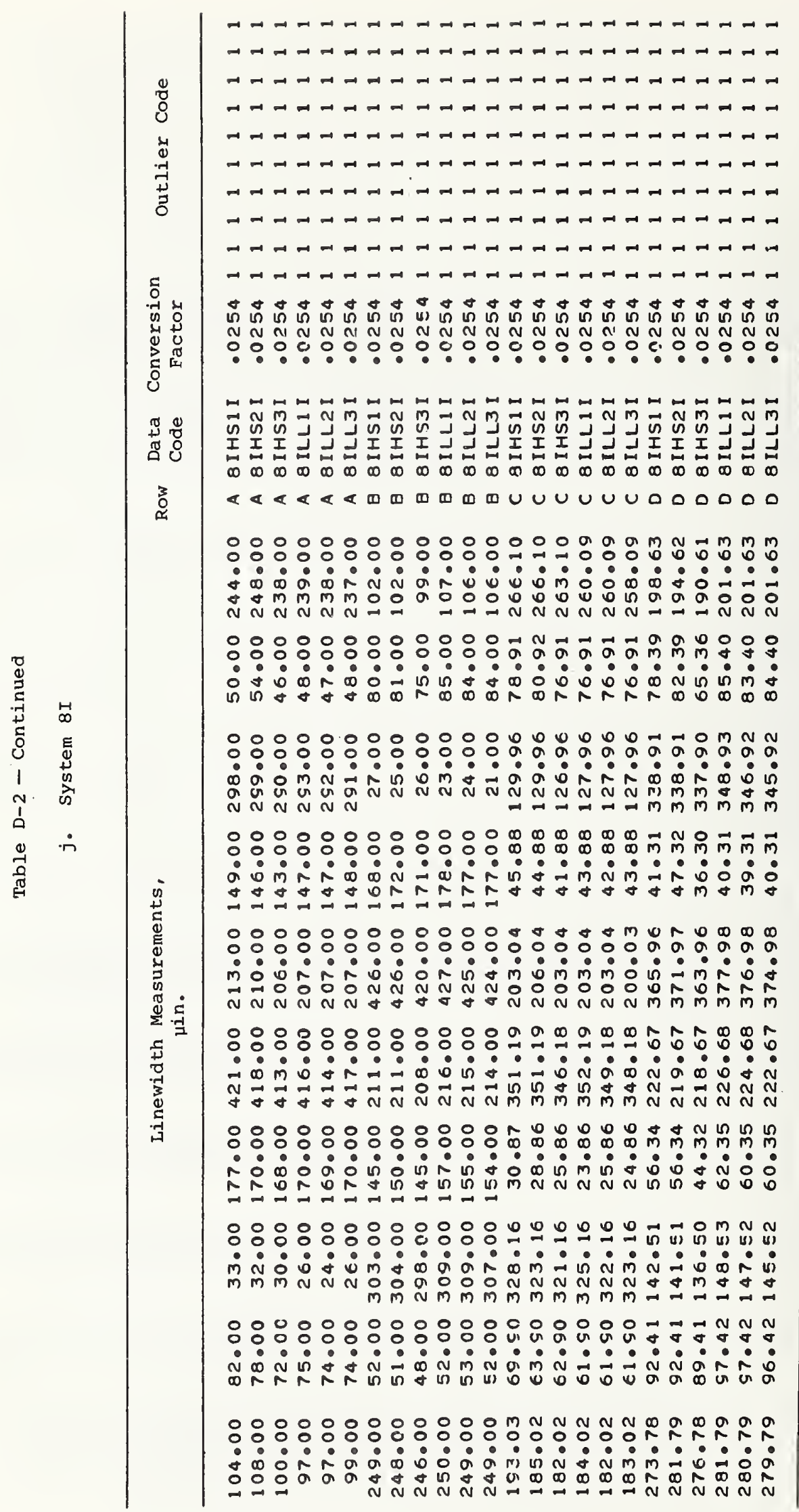




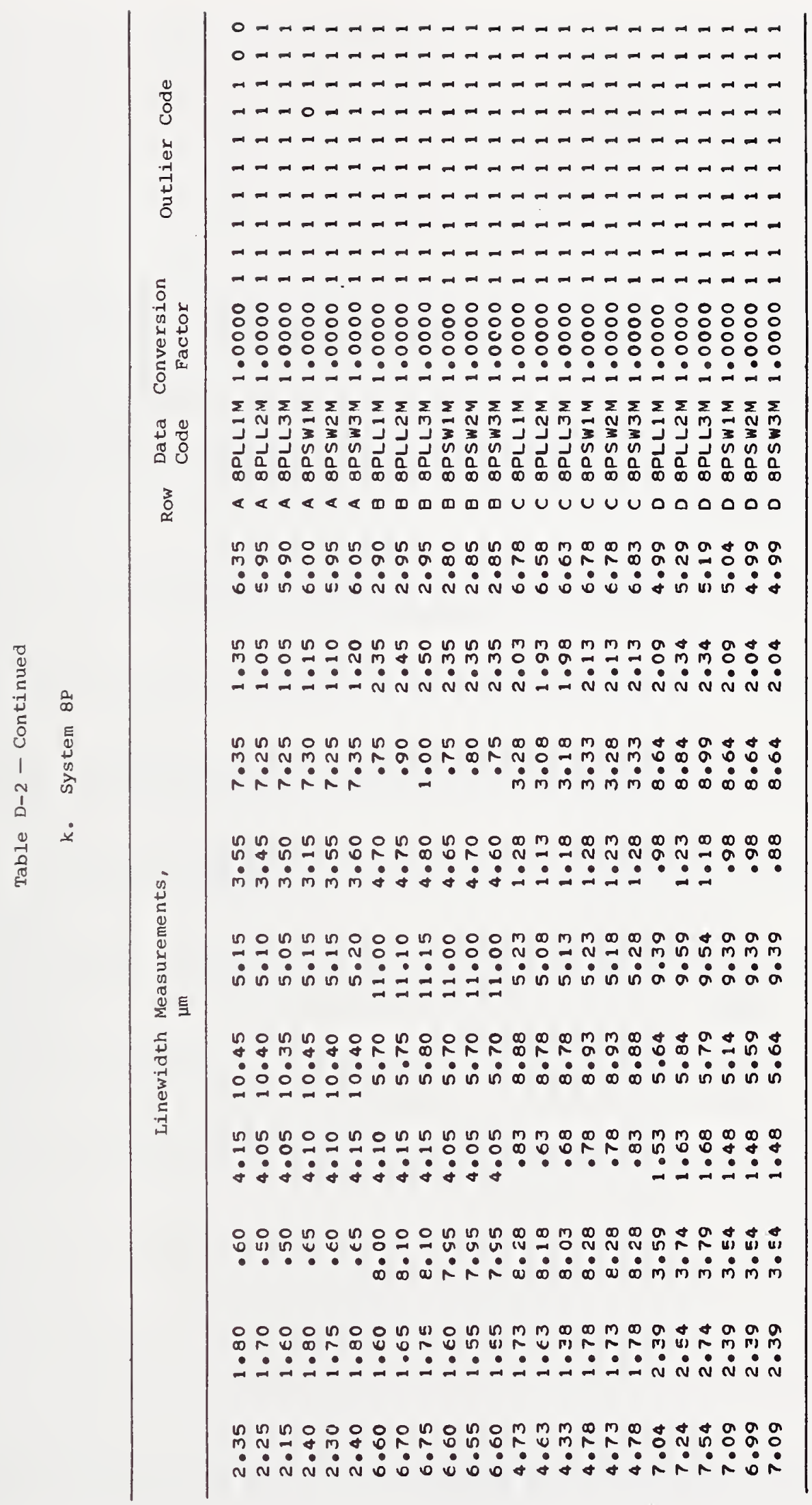




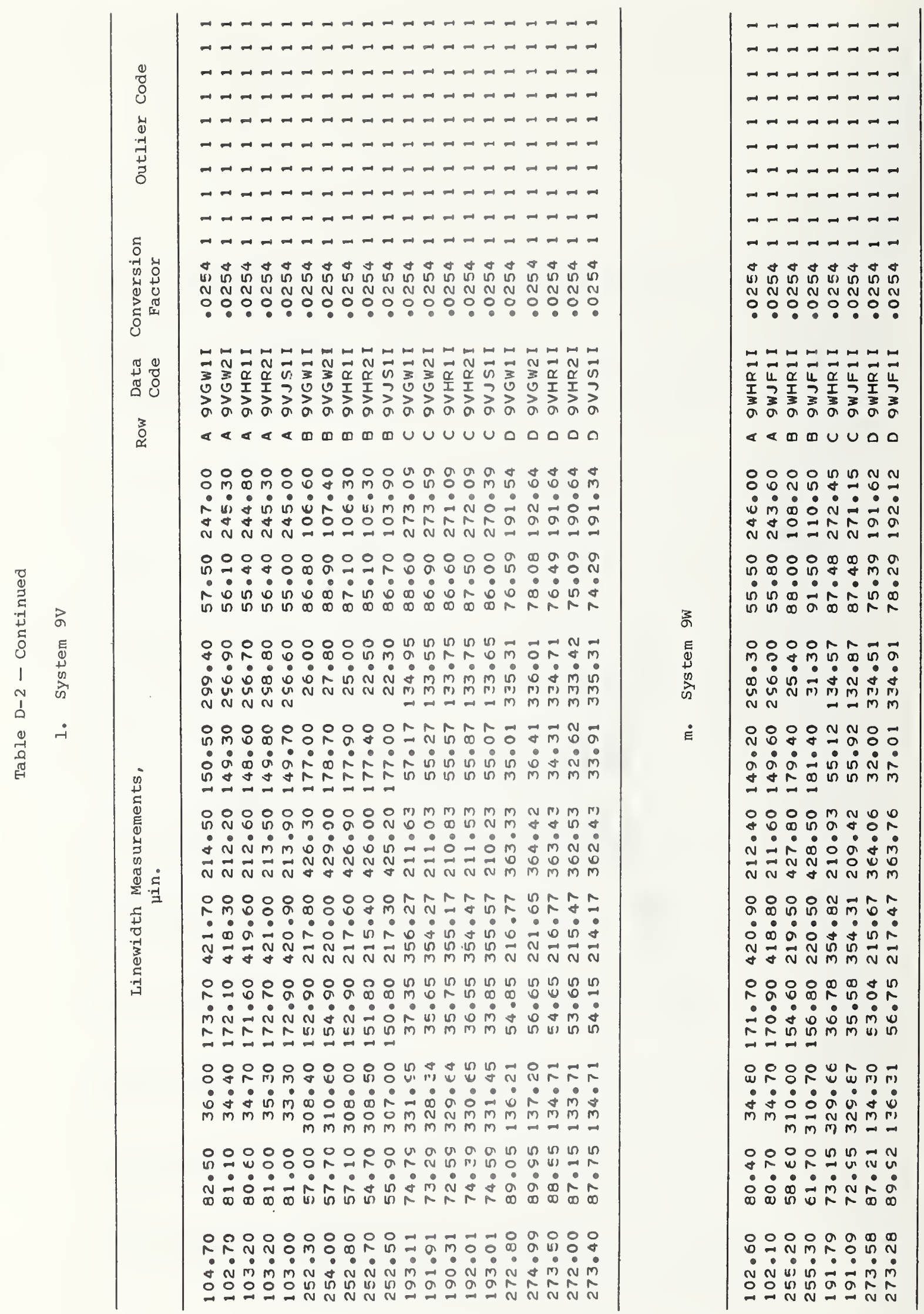


응ㅇㅇㅇㅇㅇㅇㅇㅇㅇㅇㅇㅇㅇㅇㅇㅇㅇㅇㅇㅇㅇㅇㅇㅇㅇㅇㅇㅇㅇㅇ

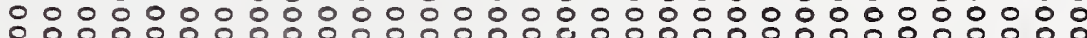
00000000000000000000000000000000

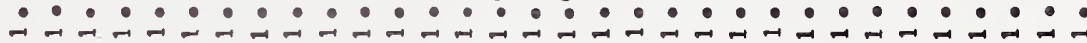
$\sum \sum \sum \sum \sum \sum \sum \sum \sum \sum \sum \sum \sum \sum \sum \sum \sum \sum \sum \sum \sum \sum \sum \sum \sum \sum \sum \sum \sum \sum \sum \sum$ ה

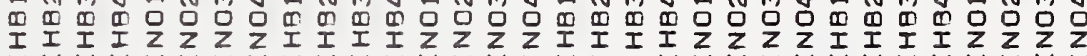
00000000000000000000000000000000

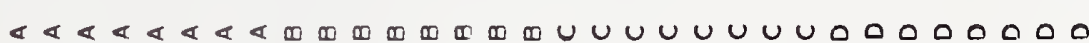

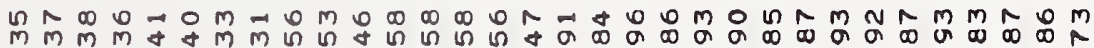
$\dot{0} \dot{0} \dot{0} \dot{0} \dot{0} \dot{0} \dot{N} \dot{N} \dot{N} \dot{N} \dot{N} \dot{N} \dot{0} \dot{0} \dot{0} \dot{0} \dot{0} \dot{0} \dot{0} \dot{+} \dot{q} \dot{+} \dot{q} \dot{q} \dot{+} \dot{+}$

mot -

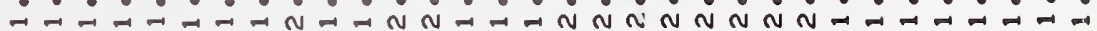

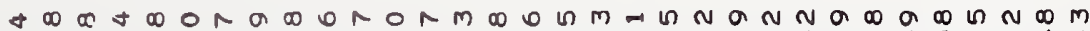

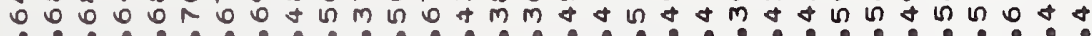

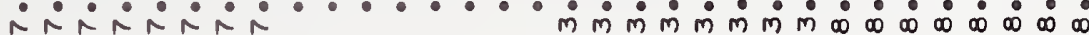

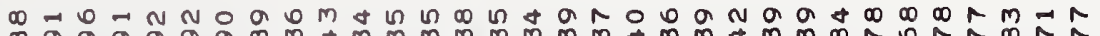
.

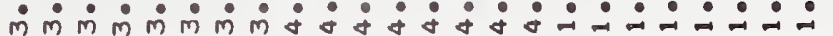

○

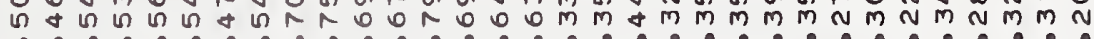

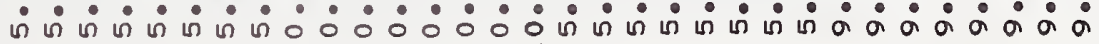

m ?

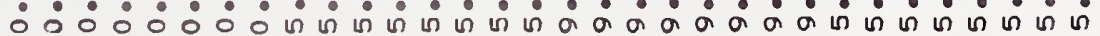

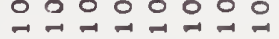

a 4. $\dot{q} \dot{+} \dot{\sigma} \dot{\sigma} \dot{q} \dot{\theta} \dot{q} \dot{m} \dot{m} \dot{m} \dot{m} \dot{m}$

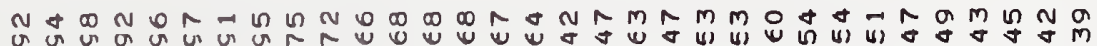
.........

$n+a \wedge \wedge n 000$ ก

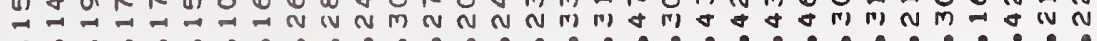
$\dot{N} \dot{N} \dot{N} \dot{N} \dot{N}-\dot{H}-\dot{-}-\dot{\sim} \dot{N} \dot{N} \dot{N} \dot{N} \dot{N} \dot{N} \dot{N} \dot{N} \dot{N} \dot{N} \dot{N} \dot{N}$

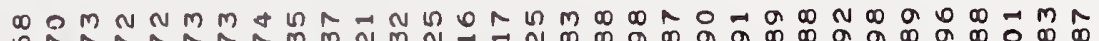

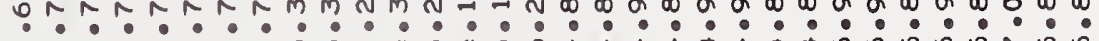

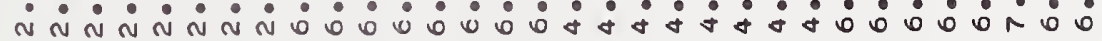




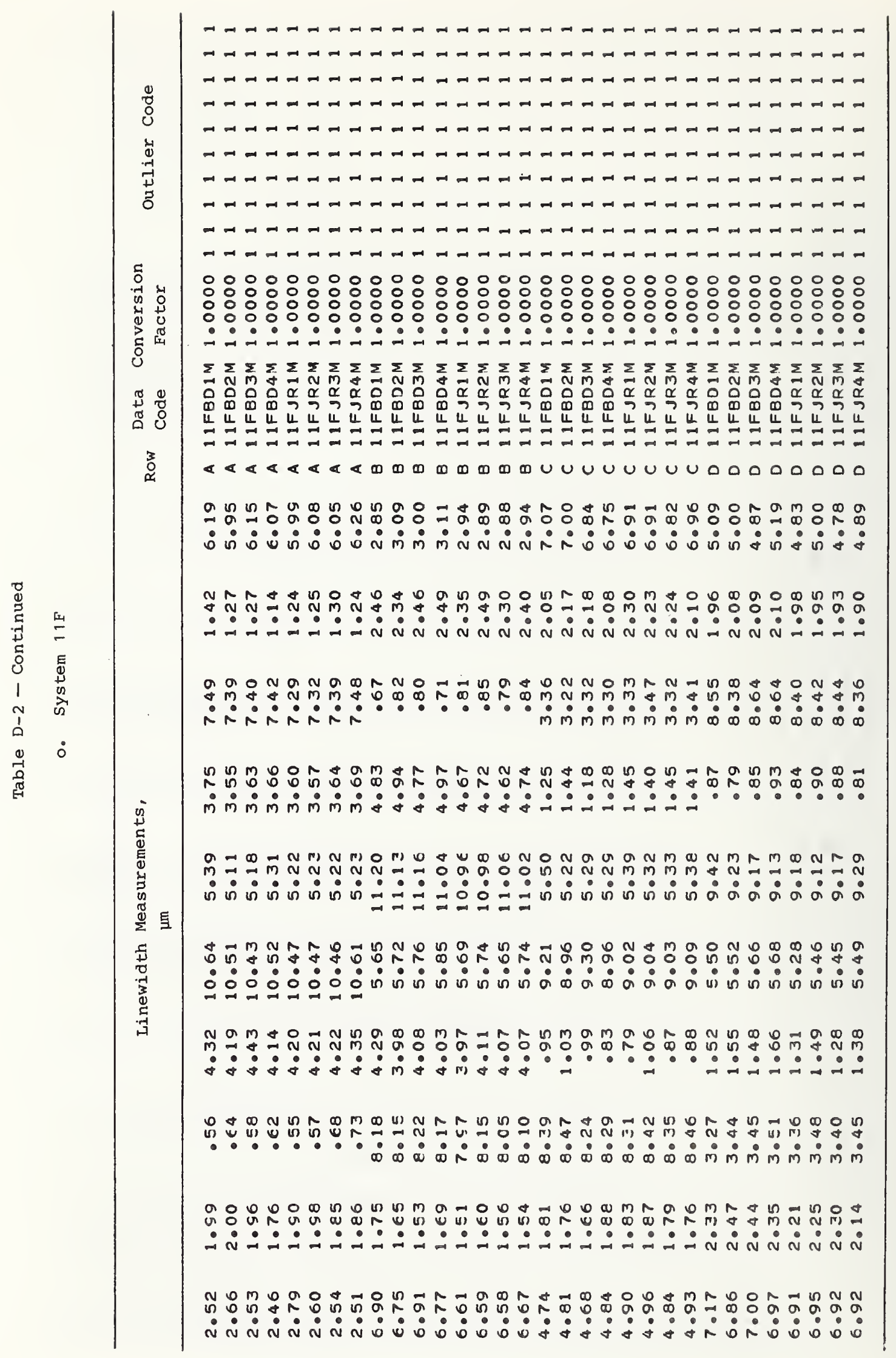




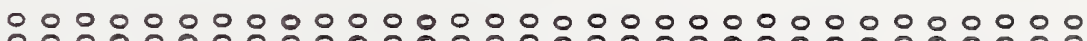

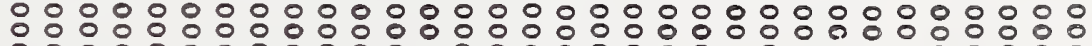
00000000000000000000000000000000

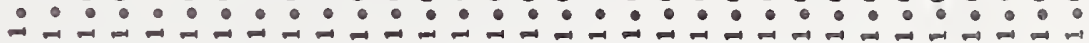

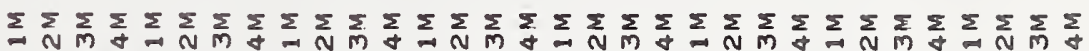
जี 芷

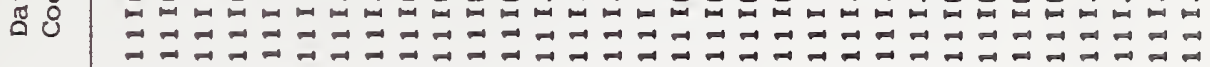

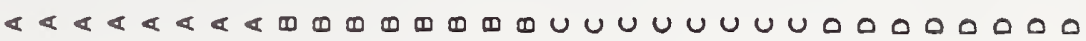

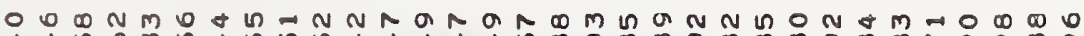

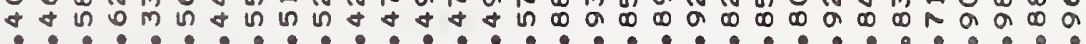
$\dot{0} \dot{0} \dot{0} \dot{0} \dot{0} \dot{0} \dot{N} \dot{N} \dot{N} \dot{N} \dot{N} \dot{N} \dot{0} \dot{0} \dot{0} \dot{0} \dot{0} \dot{0} \dot{0} \dot{+} \dot{+} \dot{+} \dot{+} \dot{+} \dot{+} \dot{+}$

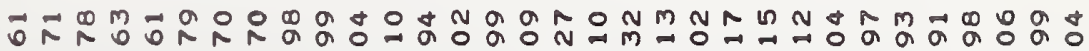

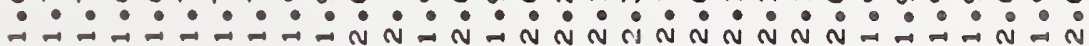

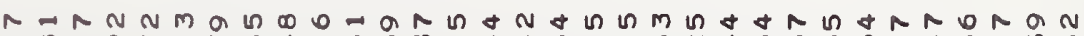
m. ininini

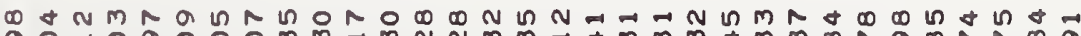

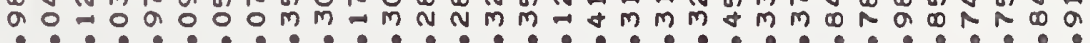

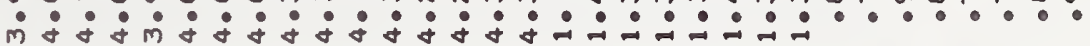

notoromodn

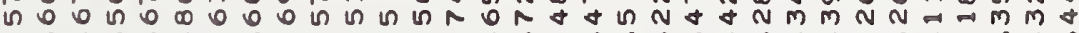
ம

ก⿻上丨

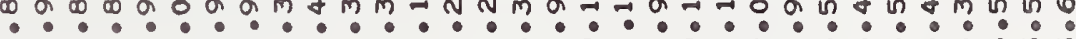
$\circ 0000=00$ uो ம

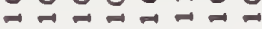

苟

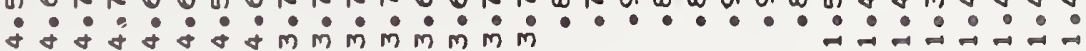

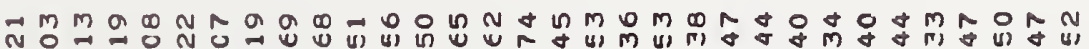
ก

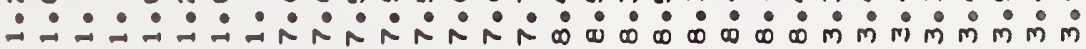

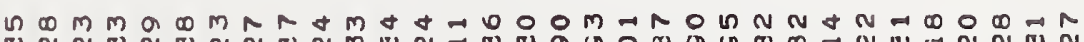

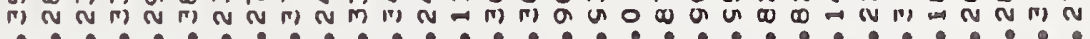

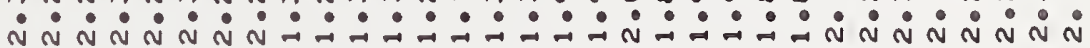

이에

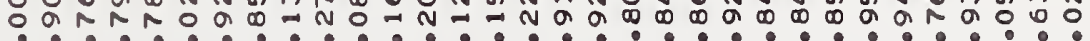

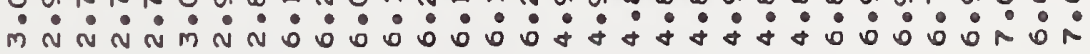




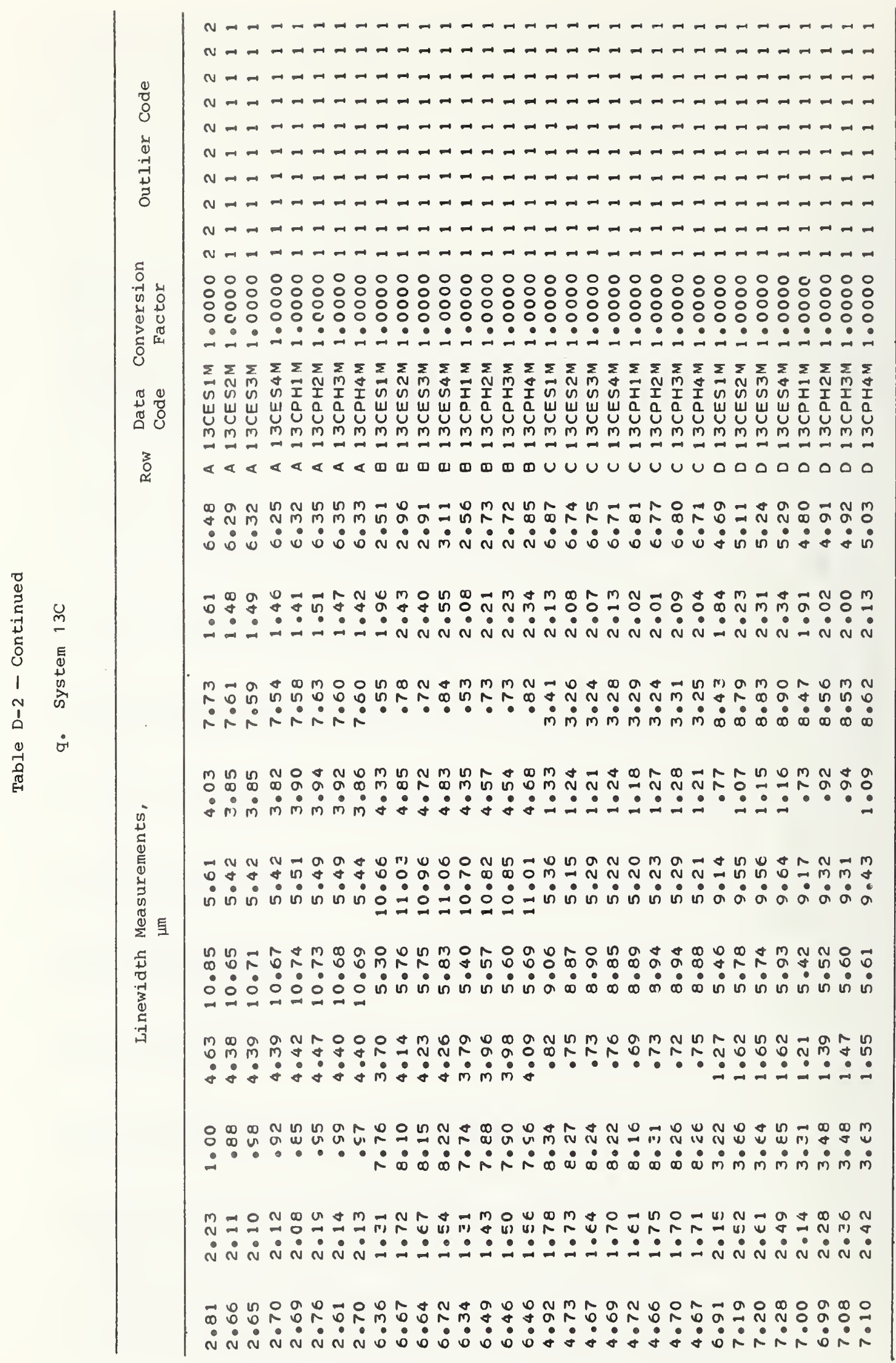




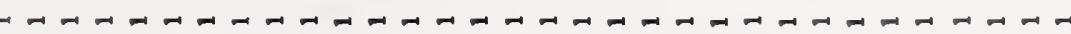

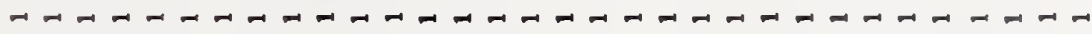

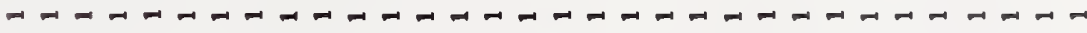

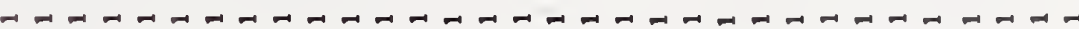

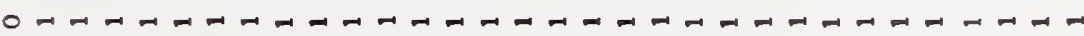

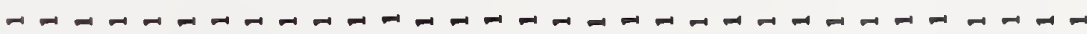

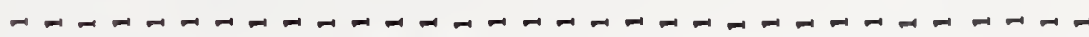

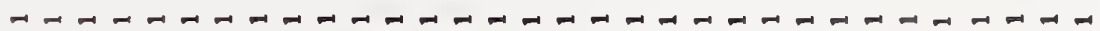

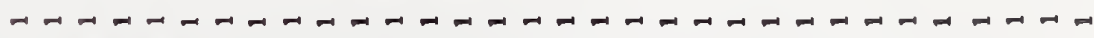

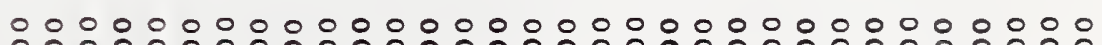

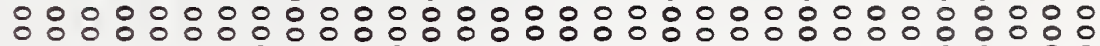

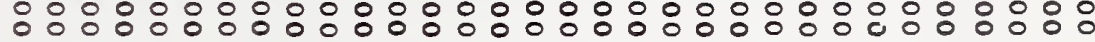

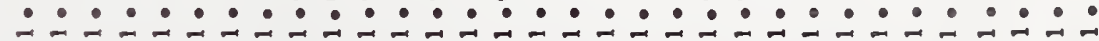

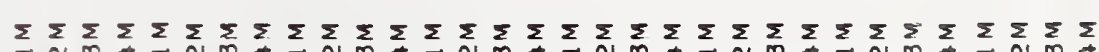

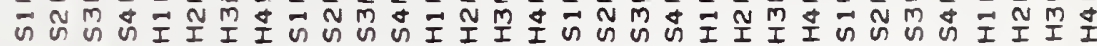

。

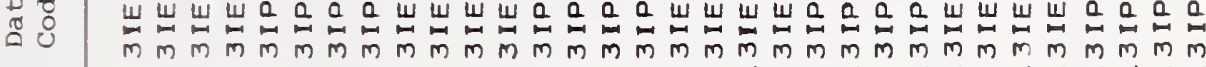

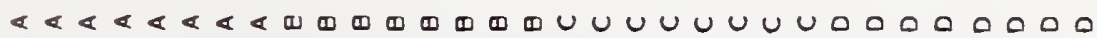

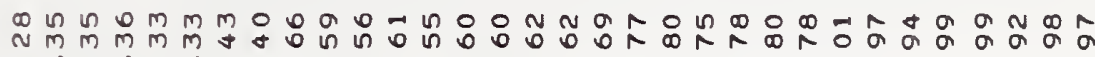

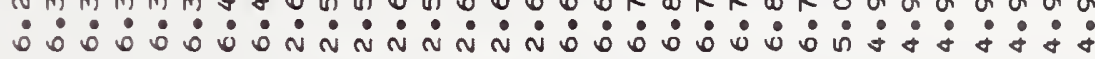

กตน \&

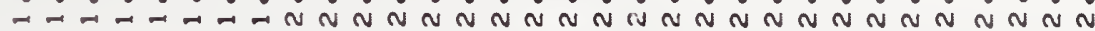

o o on

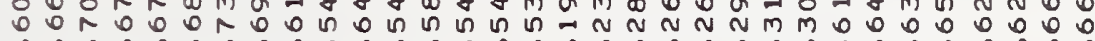
$\dot{\sim} \dot{r} \dot{i} \dot{i} \dot{r} \cdot \cdots \dot{m} \dot{m} \dot{m} \dot{m} \dot{m} \dot{m} \dot{m} \dot{\infty} \dot{\infty} \dot{\infty} \dot{\infty} \dot{\infty} \dot{\infty} \dot{\infty}$

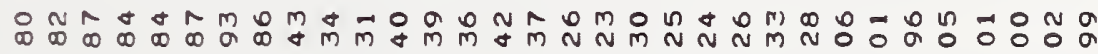

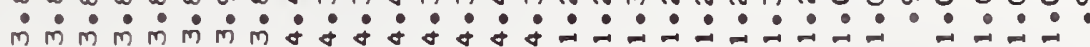

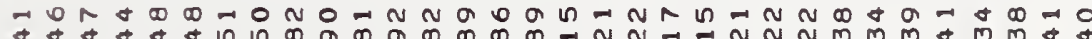

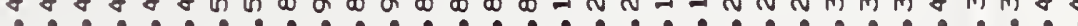

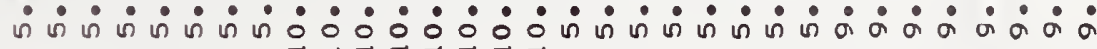

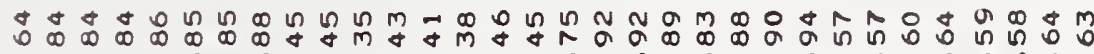

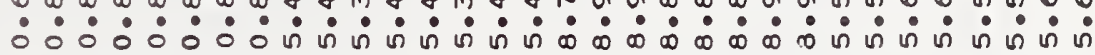
$\therefore-1-1-1=$

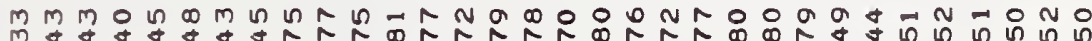

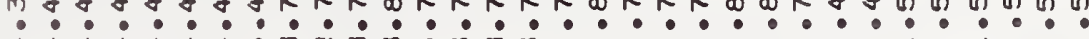

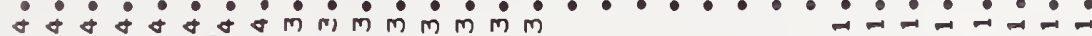

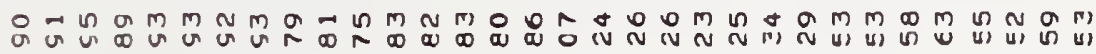

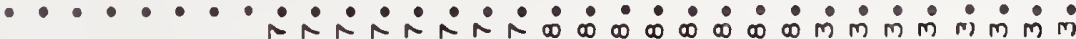

an uี

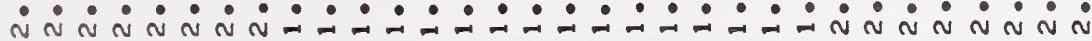

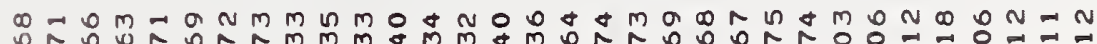

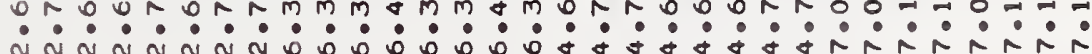




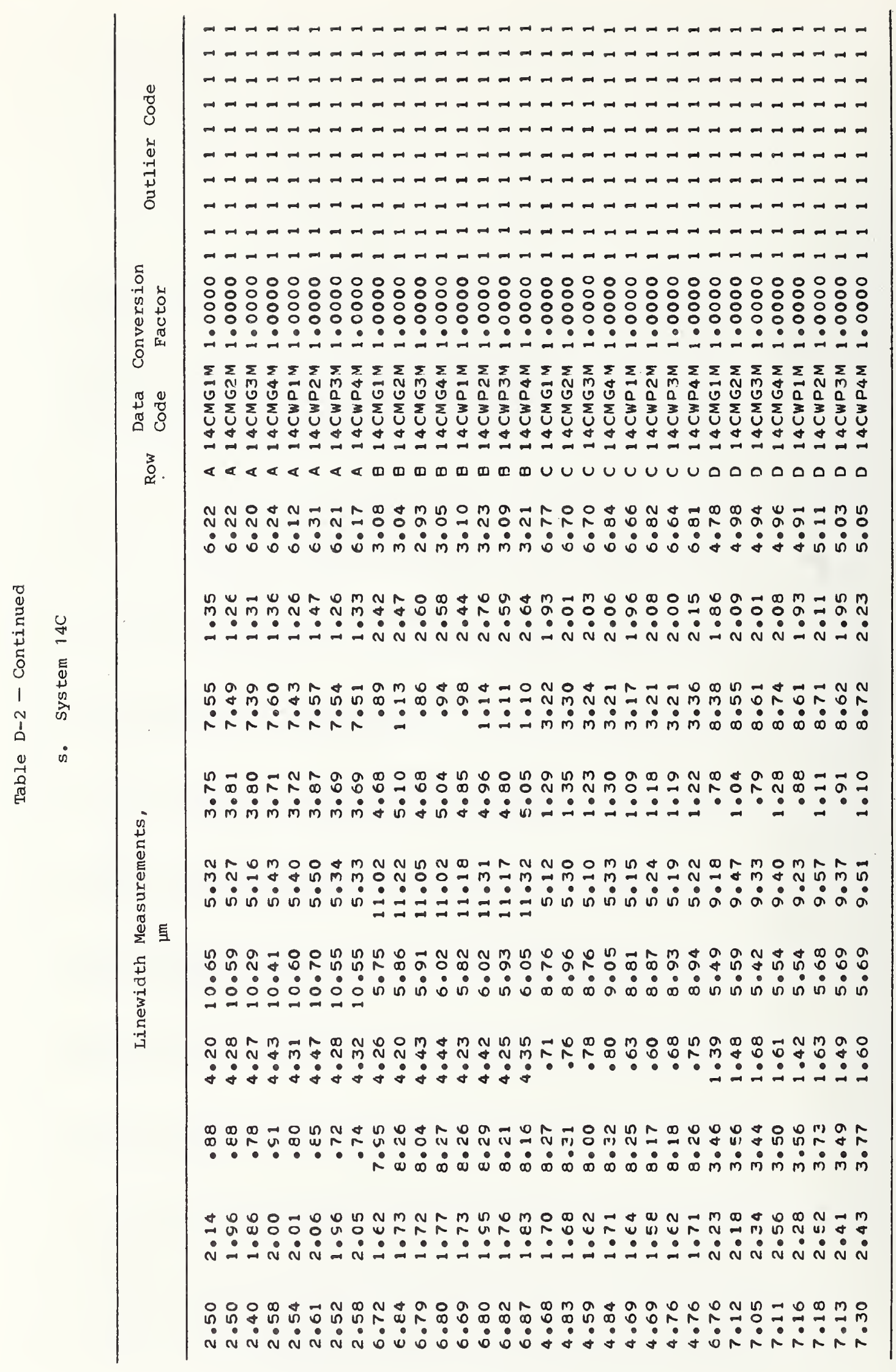




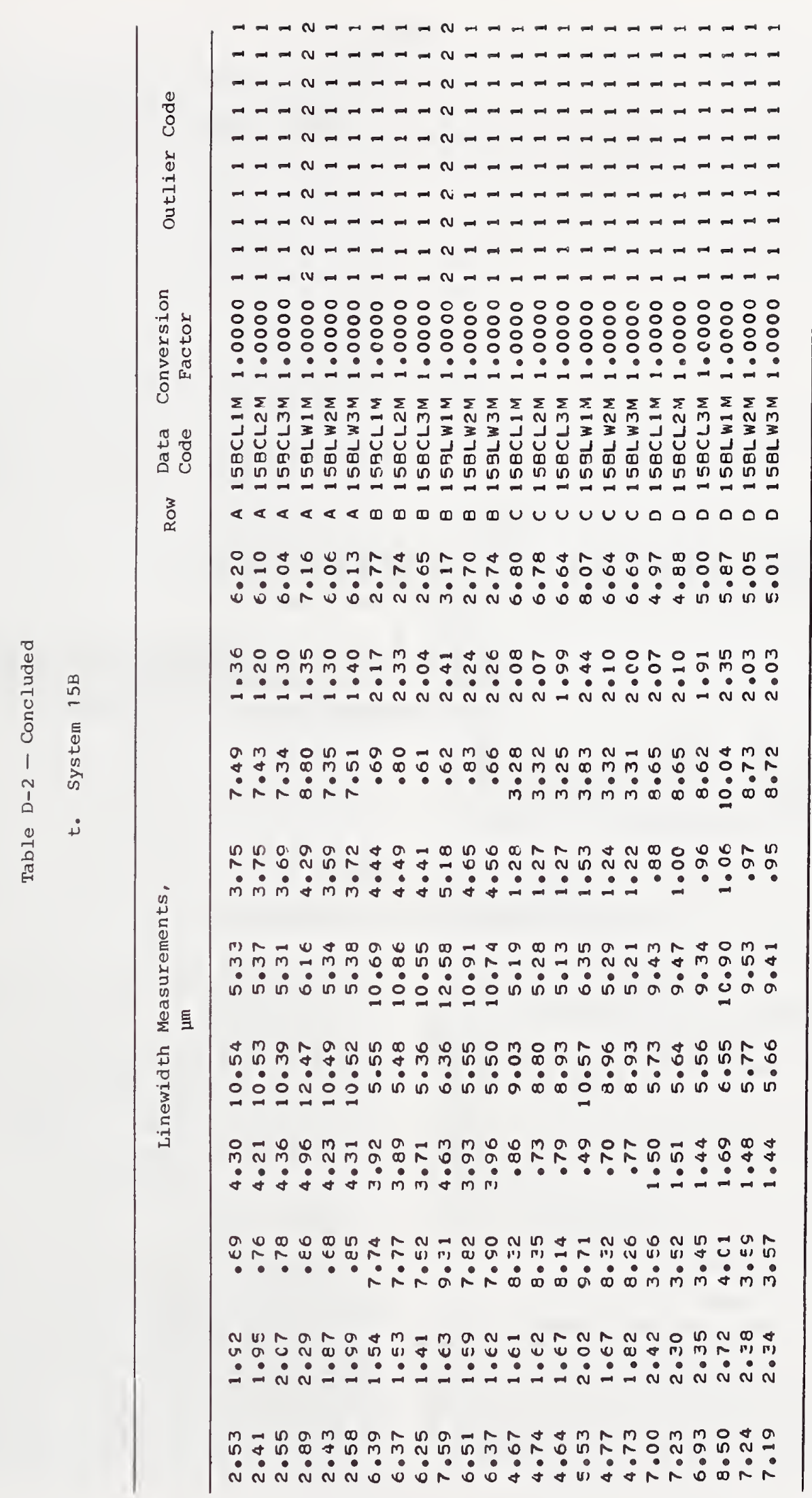




\section{Graphical Diagnosis of Interlaboratory}

\section{Test Results

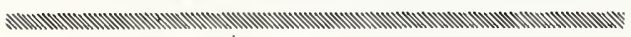

\section{W. J. YOUDEN}

National Bureau of Standards, Washington, D. C.

\section{Introduction}

Interlaboratory or round robin programs to evaluate the performance of test procedures will always be with us. New materials require new tests. New, and hopefully better, test procedures are developed for old products. Test procedures are used to ascertain whether a product meets the specification set down for the product. A double problem confronts the producer. There is bound to be a certain amount of variation in his product. And there is bound to be variation in the test results made on a given sample of the product. The impact of the errors of measurement associated with the test procedure is obvious because half the tests made on a product that just meets specification will rate the product below specification.

\section{Test Procedures and Production Costs}

It is customary to manufacture purposely a product that exceeds specification in order to allow for testing errors. The larger these testing errors, the greater the excess quality that must be built into the product to insure the acceptance of nearly all lots that are in fact equal to or better than the specification. The manufacturer already has to contend with variation in the process. Considerable saving in manufacturing costs can be affected by reducing the margin between the quality level set for production and that called for in the specification. The savings attainable with improved test procedures are a strong inducement for the improvement of test procedures. Interlaboratory test programs of varying degrees of thoroughness are frequently used to establish the performance of existing procedures.

\section{Missed Opportunities in Interlaboratory Test Programs}

Strangely enough modern statistical tests such as the analysis of multifactor studies and the isolation of components of variance have not made the contribution expected of them. Part of this no doubt comes about because these more sophisticated statistical techniques are not too well understood by some of those in the laboratories that run the tests. It is all very well for someone with statistical skill to set up an intricate interlaboratory test program and analyse the data but this still leaves the problem of interpreting the statistical jargon to those directly concerned. Even when this interpretation is undertaken the report is apt to read somewhat along these lines. "Duplicates run by the same operator in the 
same laboratory show excellent agreement. Agreement between different operators in the same laboratories is not quite so good, and very poor between results from different laboratories. Results on different days do not agree as well as those obtained on the same day." This is a brief summary of the interpretation that is made after the statistical analysis shows that practically all the F-tests are significant. Unhappily almost all concerned were already aware of the state of affairs just described and want to know what can be done to improve matters. It is just here that statisticians have not risen to the opportunities presented by interlaboratory test programs.

When all is said and done, what we want is rather simple. We want to know whether the test procedure as set forth is capable of yielding acceptable agreement among results from different laboratories. If the results are not acceptable, we would like some specific indication of what is wrong with the procedure. If the procedure appears to be reasonably good but there are some disturbing discrepancies, we would like to know which laboratories are having trouble and if possible why they are having trouble. And most important we should be able to get this information back to the laboratories concerned in such a form that the diagnosis is believed. For only so will these laboratories take any action to correct the difficulties.

\section{Graphical Representation of Results}

The graphical procedure is based upon a very simple interlaboratory program. Samples of two different materials, $\mathrm{A}$ and $\mathrm{B}$, are sent to a number of laboratories which are asked to make one test on each material. The two materials should be similar and be reasonably close in the magnitude of the property evaluated. This will avoid complications that may arise from differential behavior of the two test materials. A second pair of samples are circulated at a later time if there are only a few participating laboratories. The pairs of results that are reported by the laboratories are used to prepare a graph.

The graph is prepared by drawing the customary $\mathrm{x}-$ axis at the bottom of the paper and laying off on this axis a scale that covers the range of results for material A. At the left the $y$-axis is provided with a scale in the same units that includes the range of results reported for material B. The pair of results reported by a laboratory are then used to plot a point. There will be as many points as there are reporting laboratories. After the points are plotted a horizontal median line is drawn parallel to the $x$-axis so that there are as many points above the line as there are below it. A second median line is drawn parallel to the $y$-axis and so placed that there are as many points on the left as there are on the right of this line. Figure 1 shows the seven-day tensile strengths reported by 25 laboratories on two cement samples. Two of the laboratories are so patently separated from the other 23 that they are not used in determining the position of the median lines.

\section{Diagnosis of the Configuration of Points}

The two median lines divide the graph paper into four quadrants. In the ideal situation where only random errors of precision operate the points are expected to be equally numerous in all quadrants. This follows because plus and minus errors should be equally likely. In any existing test procedure that has come to my attention the points tend to concentrate in the upper right and lower left quadrants. This means that laboratories tend to get high results on both materials or low results on both materials. Here is evidence of individual laboratory

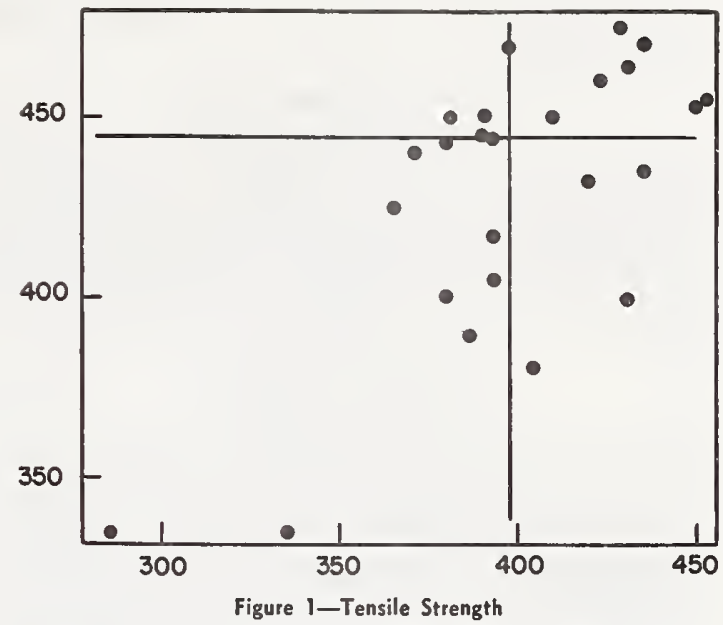

biases. There is evidence of this state of affairs in Fig. 1. The more pronounced this tendency to individual bias the greater the departure from the expected circular distribution of points about the intersection of the median lines.

Figure 2 shows 15 points plotted from phthalic anhydride determinations on two paint samples. The points tend to scatter more or less closely along a line approximately bisecting the upper right and lower left quadrants. There is reason to expect the line to make a 45 degree angle with the axes when the same scale is used for both axes and the two materials are sufficiently similar so that the dispersion of the results is about the same for each material.

A test procedure that yields results like those in Fig. 2 is probably in need of more careful description. In its present form the procedure apparently is open to individual modifications that do have an effect upon the results. The procedure rather than the laboratories should be considered as a possible source of the difficulty even though the difficulty is exhibited by a large scatter among the results from the different laboratories. When the points lie closely along the 45 degree line the conclusion may be drawn that many of the laboratories

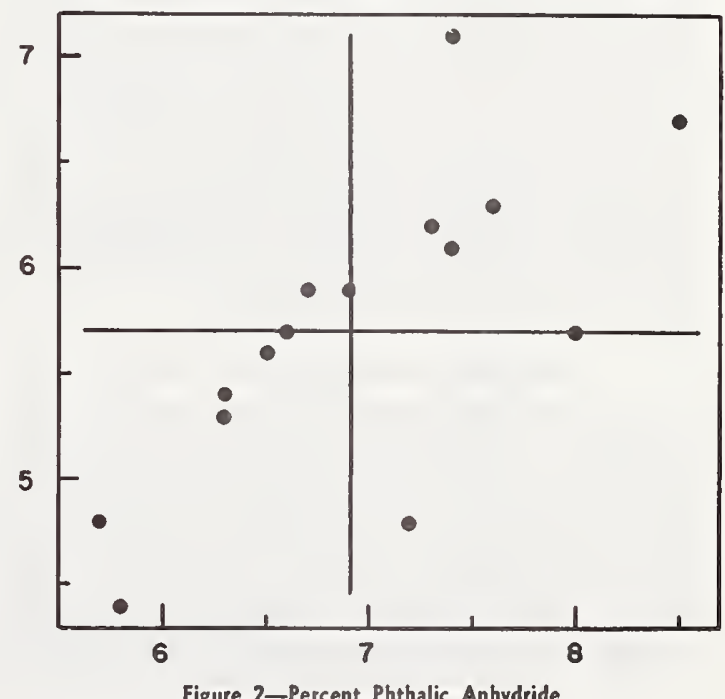

Figure 2-Percent Phthalic Anhydride 
are following rather carefully their own versions of the test procedure.

\section{Checking on Sample Variation}

There is no possibility of the distribution of points in Fig. 2 arising from lack of uniformity among the samples distributed from each material. If the stock is heterogeneous, some samples will be high, some low, and this will be true for both materials. The pairs of samples distributed to the laboratories will be of four kinds:

high in A, high in $B$

high in $A$, low in $B$

low in $A$, high in $B$

low in $A$, low in $B$

The four possible combinations have the same probability of occurrence and would result in the test results being nearly equally divided among the four quadrants. Concentration of the points in two quadrants rules out questions of sampling heterogeneity.

On the other hand if there is a roughly circular distribution of points but with a disappointingly widespread scatter, the diagram does not reveal whether this arises from sampling difficulties or poor precision of the test results. If sampling is considered a possible source of difficulty the following modification in the assignment of samples should be tried. If there are $2 \mathrm{~N}$ laboratories, prepare $\mathrm{N}$ double-size samples for each material. Carefully mix and divide each double-size sample into two usual size samples.

\begin{tabular}{|c|c|c|c|}
\hline Double size & & & les \\
\hline sample & Laboratory & A & B \\
\hline 1 & $\left\{\begin{array}{l}1 \\
2\end{array}\right.$ & $\begin{array}{l}1 \mathrm{~A} \\
1 \mathrm{~A}^{\prime}\end{array}$ & $\begin{array}{l}\text { 1B } \\
1 \mathrm{~B}^{\prime}\end{array}$ \\
\hline 2 & $\begin{array}{l}3 \\
4\end{array}$ & $\begin{array}{l}2 \mathrm{~A} \\
2 \mathrm{~A}^{\prime}\end{array}$ & $\begin{array}{l}2 B \\
2 B^{\prime}\end{array}$ \\
\hline $\mathrm{N}$ & $\left\{\begin{array}{l}2 \mathrm{~N}-1 \\
2 \mathrm{~N}\end{array}\right.$ & $\begin{array}{l}\text { NA } \\
\text { NA' }^{\prime}\end{array}$ & $\begin{array}{l}\text { NB } \\
\text { NB' }^{\prime}\end{array}$ \\
\hline
\end{tabular}

The samples are assigned to laboratories as shown above. It should be possible to mix and divide each double-size sample into two closely matching regular samples. These samples are assigned to a pair of laboratories. If there are sampling difficulties the plotted points should tend to occur in doublets. Two laboratories getting the two carefully mixed halves should check each other and have their points close together. This involves a little extra work in getting out the samples and no extra work for the participating laboratories. If the points corresponding to the two halves of a double-size sample are separated as much, on the average, as points from different double samples, the dispersion cannot be ascribed to sampling. In addition to noting the spacial distribution the projections of the points on the axes may also be used to see whether just one of the materials was heterogeneous.

\section{Interpretation of Out-of-Line Results}

So far the large aspects of the diagram have been examined. The individual points can now be considered and in particular those points most distant from the intersection of the median lines. Almost always one or more points are so far out of the picture that it is better not to compress the scale in order to show them. Such points should be ignored in locating the median lines. (See Fig. 1.) The more distant points tend to fall into one or the other of two categories. Either the point is far out and remote from both axes or far out and fairly close to one or the other axis. In the latter case, the result is fairly good on one material and very bad on the other. Examples of such points are found in Figures 2 and 3 . Often the explanation is simple-a mistake in typing, or calculation, or some simple blunder that sometimes can be corrected by going back to the records. If the same laboratory shows up in such a manner on succeeding pairs of materials, this implies carelessness on the part of the laboratory. The laboratory can do good work but often does not. Occasionally a laboratory has difficulty with one material and not with the other but this is not likely to occur with similar materials.

Points in the upper right or lower left quadrants that are far removed from the intersection of the median lines and that are not near either axis reflect a tendency to get either high results on both materials or low results on both materials. There are examples in all the figures. The more consistent a laboratory is in its work the more likely its point will lie in the proximity of the 45 degree line. A point far out along this line suggests the possibility that the laboratory concerned has introduced some modification in to the test procedure. A laboratory finding itself in this situation should check carefully the prescribed procedure for performing the test and endeavor to locate the cause of the large bias.

All of the above interpretation can be made while keeping anonymous the identity of the plotted points. When circulating a report of the interlaboratory test it might be helpful to circle in red the point belonging to the laboratory in the copy going to that laboratory. That would save the laboratory from consulting its files to locate itself and would display prominently just where the laboratory stood in reference to the whole group. This vivid picturing of a laboratory's position should stimulate the laboratory to some self examination that could hardly avoid having beneficial results.

\section{Estimating the Precision of the Test Procedure}

The above discussion does not exhaust the information to be gleaned from this graphical representation. Assuming that the two materials are similar in type and nearly equal in magnitude for the property the dispersion among the results reported for $A$ should be about the same as the dispersion of the $B$ results. In that event the 45 degree line through the intersection of the medians makes possible an estimate of the precision of the data. Often an interlaboratory test undertakes to differentiate among the laboratories in respect to precision. Not only does this require large numbers of measurements from each laboratory but differences in precision usually turn out to be unimportant in comparison with bias errors and careless errors. No violence at this stage seems to be done by assuming about the same precision for all the laboratories.

The perpendicular distance from each point to the $\mathbf{4 5}$ degree line can be used to form an estimate of the precision. The estimate of the standard deviation of a single result is obtained by multiplying the average length of the perpendiculars by $\sqrt{\pi / 2}$ or 1.2533 . These perpendiculars need not be measured on the graph paper. Instead, write $d$ wn for each laboratory the difference (A-B) keeping track of the signs. Call these differences $d_{1}, d_{2}, \ldots d_{n}$. Calculate $\bar{d}$, the algebraic average difference. Subtract $\bar{d}$ from each difference and obtain a set of corrected differences $d_{1}{ }^{\prime}, d_{2}^{\prime}, \ldots d_{n}{ }^{\prime}$. The average of the absolute values of these differences when multiplied by $\sqrt{\pi} / 2$ or 0.886 gives an estimate of the standard deviation. 
TABLE -Data and Calculations on Percent Insoluble Residue in Cement Reported by 29 Laboratories

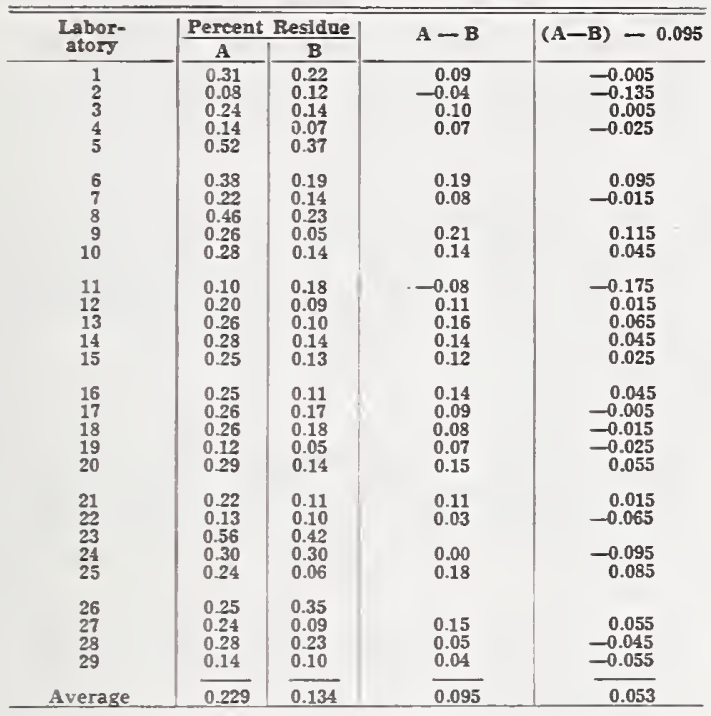

The data on percent insoluble residues reported by 29 laboratories are given in Table I and plotted in Fig. 3. There are three points far out along the 45 degree line and one far out on the $y$-axis. These laboratories were excluded from the calculations shown in Table I. The last column shows the differences between the two results diminished by the difference between the two sample averages. The average, 0.053 , shown at the bottom of this column is the average of the absolute values, ie., ignoring the signs. Multiplying 0.053 by 0.886 gives 0.047 as the estimate for the standard deviation of a single result. Probably this is inflated by leaving in the two laboratories turning in the very low results for samole A.

This estimate of the standard deviation for precision leads to the construction of circles (centered on the intersection of the median lines) within which any given percentage of the points can be expected to fall should the laboratories be able to eliminate all bias or constant errors. The multiples of the standard deviation that include various percents of the points are given in Table II.

Thus a circle whose radius is about 2.5 to 3.0 times the standard deviation gives a fair idea of the smallest circle that could be expected to contain nearly all points after the elimination of the constant errors that are causing the points to congregate in the upper left and lower right quadrants. Generally a fair number of points will lie outside such a circle. The laboratories respon-

TABLE II-Probability Table for Circular Normal Distribution

\begin{tabular}{c|c}
\hline \hline $\begin{array}{c}\text { Percent of the Points } \\
\text { Within Circle }\end{array}$ & $\begin{array}{c}\text { Multiple b of the } \\
\text { Standard Deviation }\end{array}$ \\
\hline 10 & 0.459 \\
20 & 0.668 \\
25 & 0.759 \\
30 & 0.845 \\
40 & 1.011 \\
50 & 1.177 \\
60 & 1.350 \\
70 & 1.552 \\
75 & 1.665 \\
80 & 1.794 \\
90 & 2.146 \\
95 & 3.448 \\
99 & 3.035 \\
\hline
\end{tabular}

Note: Percent $=100\left[1-\exp \left(-\mathrm{b}^{2} / 2\right)\right]$

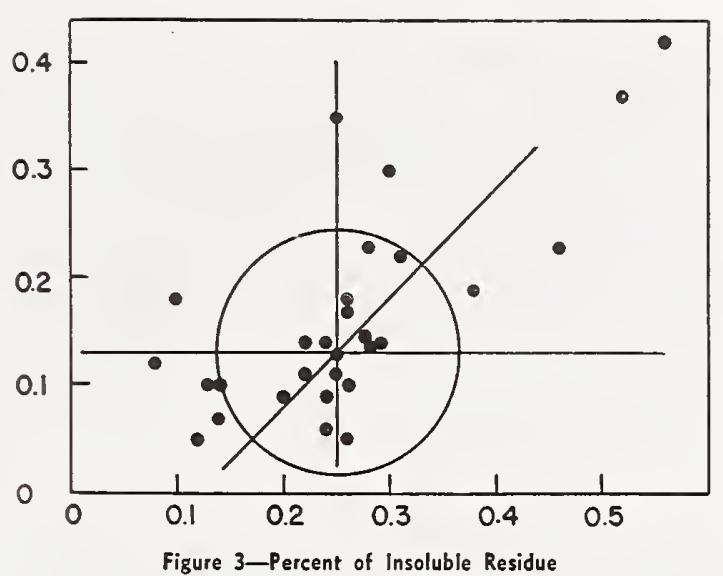

sible for these points almost certainly have somehow got substantial systematic errors incorporated in their techniques. Multiplying the standard deviation obtained above by 2.45 gives the radius of the circle that should include 95 percent of the laboratories if individual constan errors could be eliminated. This circle is drawn in Fig. 3. Seven further laboratories are outside the circle including the two who got the benefit of the doubt and were retained in the computation. This examination has directed attention to at least six of the laboratories that might well go over their method of making this determination of insoluble residue.

If the number of laboratories in the program is rather small, the way to accumulate more points is to send the laboratories additional pairs of samples from different materials. A chart is prepared for each pair of materials and the median lines drawn in. The charts are now superimposed so that the points of intersection of the median lines coincide and, of course, the median lines also. All points are then transferred to one sheet of paper with one pair of median lines. As there are only a few laboratories each can be assigned an identitying symbol.

Figure 4 shows the reports made by eight labortories determining $\mathrm{CaO}$ in cement. The laboratories are

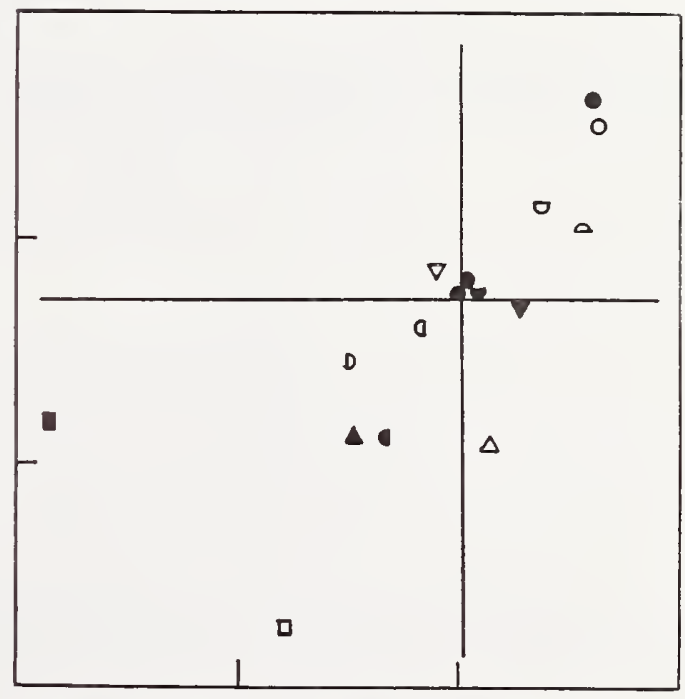

Figure $4-\mathrm{CaO}$ in Cement (interval equals one percent)

179 
identified by symbols. The hollow symbols show the results for the first pair of samples. The corresponding solid symbols show the work of these same laboratories on a second pair of samples. Few as these data are they serve to indicate the things that we want to know about the test procedure and about the laboratories. Clearly this procedure is one that is vulnerable to individual bias. 'Two of the eight laboratories appear in the same region for both pairs. The circle laboratory is very consistent-and gets the highest results. The square laboratory gets very low results and is not very precise as shown by the fact that the two squares are separated by a much greater distance than any of the other seven pairs. Using this chart some possibly helpful suggestions could be passed along.

\section{Discussion}

The two materials used in this double-sample program were specified to be similar in type and in the magnitude of the property measured. Sometimes the measurement errors are proportional to the magnitude under measurement and this will show up in a greater scatter of the points along one of the axes. Particular types of samples may give trouble in just some of the laboratories. The thorough study of a test method must include consideration of these possible complications. Naturally a more comprehensive interlaboratory test program will be required to explore these aspects of the test procedure. A thorough study in one laboratory usually reveals these complications.

\section{Summary of Advantages of Graphical Diagnosis}

The double-sample, graphic analysis scheme described in this article offers a number of advantages.

(1) An unusually light burden is imposed on each laboratory
(2) The graphical procedure greatly facilitates presentation of the results in a convincing manner

(3) No statistical background is required to follow the reasoning and no computations are required to demonstrate the general presence of constant errors and the gross deviations of individual laboratories

(4) A minimum of computation is imposed upon the individual collating the results

(5) The use of a circle of 2.5 or $3.0 \sigma$ radius shows the individual laboratories whether or not their method of carrying out the test has in some way become saddled with a substantial constant error

(6) Most important the direction for improvement is clearly indicated

a. A long, narrow ellipse directs attention to a more careful description of the procedure or even to the need for modification

b. Wild points far out near either axis indicate erratic work

c. Wild points far out along the 45 degree line are strong evidence of substantial deviations from the specified procedure

d. General prevalence of constant errors is indicated by a substantial proportion of the points lying outside the $2.5 \sigma$ circle

Experience has already indicated that a certain few laboratories are found too frequently in the most distant positions from the intersection of the median. Improved performance from these few laboratories may go far to restore confidence in a test procedure. There is no substitute for careful work in the laboratory. 
For the measurements $y_{i j}$ on a given line where $i$ denotes the $i$ th repetition and $j$ identifies the $j$ th line in a row, the calibration curves are of the form

$$
y_{i j}=\alpha+\beta x_{j} \quad \begin{aligned}
& i=1, \ldots, w_{j} \\
& j=1, \ldots, m^{m}
\end{aligned}
$$

where $w_{j}$ is the number of repetitions for the $j$ th line, and $x_{j}$ is the NBS linewidth value for the $j$ th line.

Let

$$
\begin{aligned}
& n=\sum_{j=1}^{m} w_{j} \\
& y_{. .}=\frac{\sum_{j=1}^{m} \sum_{i=1}^{w_{j}} y_{i j}}{n}
\end{aligned}
$$

and

$$
x=\frac{\sum_{j=1}^{m} x_{j}}{m}
$$

The least-squares estimates of the intercept $\alpha$ and slope $\beta$ are given by

$$
\hat{\alpha}=y . \hat{\beta} x \text {. }
$$

where

$$
\hat{\beta}=\frac{\sum_{j=1}^{m} \sum_{i=1}^{w_{j}}\left(x_{j}-x_{0}\right)\left(y_{i j}-y\right)}{\sum_{j=1}^{m} w_{j}\left(x_{j}-x_{0}\right)^{2}}
$$

The standard deviations of the estimates of the intercept and slope are given by 


$$
s_{\alpha}=s\left[\frac{\sum_{j=1}^{m} w_{j} x_{j}^{2}}{n \sum_{j=1}^{m} w_{j}\left(x_{j}-x_{0}\right)^{2}}\right]^{1 / 2}
$$

and

$$
s_{\beta}=\frac{s}{\left[\sum_{j=1}^{m} w_{j}\left(x_{j}-x_{0}\right)^{2}\right]^{1 / 2}}
$$

where the residual standard deviation is given by

$$
s=\left[\frac{\sum_{j=1}^{m} \sum_{i=1}^{w}\left(y_{i j}-\hat{\alpha}-\hat{\beta} x_{j}\right)^{2}}{n-2}\right]^{1 / 2}
$$

and $v=n-2$ are the degrees of freedom associated with $s$. 


\section{APPENDIX G}

Standard Deviation of the Residual

The definition of the standard deviation of a residual is facilitated by matrix notation. For $\mathrm{n}$ measurements consisting of $\mathrm{k}$ repetitions on $\mathrm{m}$ linewidths, the model

$$
Y=X \Phi
$$

corresponds to the model in section 3.3 .1 where $Y$ is the $(n \times 1)$ matrix of measurements, $\Phi$ is the $(1+k+m) \times 1$ matrix of unknown coefficients

$$
\Phi^{\prime}=\left(\gamma, \alpha_{1}, \ldots, \alpha_{k}, \beta_{1}, \ldots, \beta_{m}\right)
$$

and $x$ is an $n$ by $(1+k+m)$ matrix of ones and zeroes. For the $i$ th repetition on the $j$ th linewidth, the corresponding row of the matrix is as follows: The first element is one, the $(1+i)$ th element is one, the $(1+k+j)$ th element is one, and all other elements are zero.

The least-squares estimate of the coefficient matrix is

$$
\hat{\Phi}=\left(X^{\prime} X\right)^{-1} X^{\prime} Y
$$

and a residual from the least-squares fit is defined by

$$
\Lambda=\left(I-X\left(X^{\prime} X\right)^{-1} X^{\prime}\right) Y
$$

where $\Lambda$ is an (nx1) matrix.

The covariance of the residual matrix is the $(n \times n)$ matrix

$$
V(\Lambda)=\left(I-X\left(X^{\prime} X\right)^{-1} X^{\prime}\right) \sigma^{2}
$$

where the estimate of $\sigma^{2}$ is given by

$$
s^{2}=\frac{Y^{\prime}\left(I-X\left(X^{\prime} X\right)^{-1} X^{\prime}\right) Y}{n-P} .
$$

Thus the standard deviation of any residual, referred to as $s \delta_{j} j$ in section 3.3 .1 , is the square root of the diagonal element of the $[k \cdot(j-1)+i]$ th row of the matrix $V(\Lambda)$ where $s^{2}$ is substituted for $\sigma^{2}$. 
NBS-114A (REV. 2-8C)

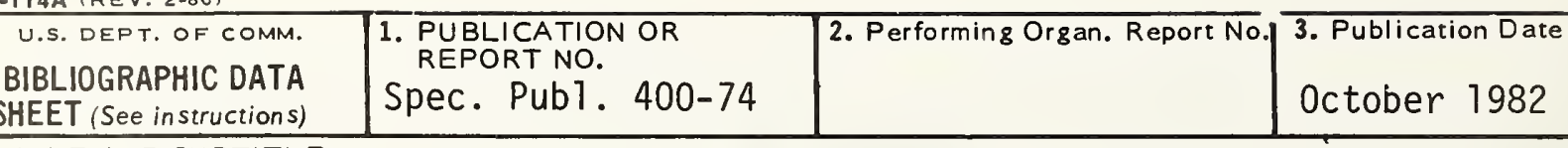

4. TITLE AND SUBTITLE

Semiconductor Measurement Technology: Interlaboratory Study on Linewidth Measurements for Antireflective Chromium Photomasks

5. AUTHOR(S)

John M: Jerke, M. Carroll Croarkin, and Ruth N. Varner

6. PERFORMING ORGANIZATION (If joint or other than NBS, see instructions)

NATIONAL BUREAU OF STANDARDS

DEPARTMENT OF COMMERCE

WASHINGTON, D.C. 20234

9. SPONSORING ORGANIZATION NAME AND COMPLETE ADDRESS (Street. City, State. ZIP)

$\begin{array}{ll}\text { Defense Advanced Research Projects } & \text { National Bureau of Standards } \\ \text { Agency } & \text { Washington, DC } 20234\end{array}$

1400 Wilson Boulevard

Ar]ington. VA 22209

10. SUPPLEMENTARY NOTES

Document describes a computer program; SF-185, FIPS Software Summary, is attached.

11. ABSTRACT (A 200-word or less factual summary of most significant information. If document includes a significant bibliography or literature survey. mention it here)

Optical microscopes fitted with a micrometer attachment are commonly used to measure small linewidths and other critical dimensions on integrated-circuit (IC) photomasks. in the absence of calibrated ilinewidth standards, users have experienced systematic measurement errors much larger than required manufacturing tolerances. As ic ilinewidths approach $1 \mathrm{~m}$ with a 10 percent tolerance, the need for calibration standards and improved measurement procedures becomes even more important.

This report discusses the resuits of an interlaboratory study to evaluate a Natlonai Bureau of Standards (NBS) prototype calibration standard for I inewidth measurements on iC photomasks and procedures for adjusting and calibrating optical-microscope systems in the 0.5- to 12-um measurement range. Using procedure: furnished by NBS, industrial participants measured iine spacings and i inewidths on NBS antirefiective-chromium artifacts. A comparison of NBS I inewidth values with participants' measurements showed that most differences were less than $\pm 0.3 \mathrm{um}$. A linewidth calibration significantiy reduced systematic errors for most systems. Outliers in the data showed the need for measurement-control procedures. The standard deviation of the measurement process was $\pm 0.1 \mathrm{~m}$ or larger for about half of the systems. For some systems, there were significant day-to-day differences and operator differences.

The study showed that the NBS artifact and recommended procedures were adequate for calibrating an opticaimicroscope system which was in a state of statistical control. This study ied to the issuance of NiS Standard Reference Materlal 474 (Opticai Microscope Linewidth-Measurement Standard).

12. KEY WORDS (Six to twelve entries; alphabetical order; capitalize only proper names; and separate key words by semicolons) dimensional measurements; filar micrometer; image-shearing micrometer; integrated circuits; interlaboratory study; Kohier illumination; i ine-spacing measurements; I inewidth cailibration; ilnewidth measurements; measurement uncertainty; micrometrology; optical microscope; photomask; semiconductor technoiogy; statistical tests.

13. AVAILABILITY

X Unlimited

For Official Distribution. Do Not Release to NTIS PRINTED PAGES

[D Order From Superintendent of Documents, U.S. Government Printing Office, Washington, D.C. 20402.

Order From National Technical Information Service (NTIS), Springfield, VA. 2216I

14. NO. OF

191

15. Price 


\section{Announcement of Semiconductor Measurement Technology \\ List of Publications 72 - 1962-1981}

Elaine C. Cohen

Information Assistant

Semicsnductor Materials and Processes Division

National Bureau of Standards

Bldg. 225, Room A305

Washington, DC 20234

Dear Mrs. Cohen:

Please send a copy of your latest "Semiconductor Measurement Technology, List of Publications 72."

Name

Company

Address

City

State

Zip Code 



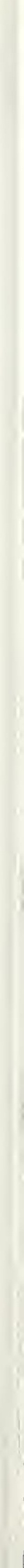







\section{NBS TECHNICAL PUBLICATIONS}

\section{PERIODICALS}

JOLRVAL OF RESEARCH-The Journal of Research of the Vational Bureau of Standards reports NBS research and development in those disciplines of the physical and engineering sciences in which the Bureau is active. These include physics, chemistry, engineering. mathematics, and computer sciences. Papers cover a broad range of subjects, with major emphasis on measurement methodology and the basic technology underlying standardization. Also included from time 10 time are survey articles on topics closely related to the Bureau's technical and scientific programs. As a spectal service 10 subscribers each issue contains complete citations to all recent Bureau publications in both NBS and nonVBS media. Issued six umes a year. Annual subscription: domestic $\$ 18$, foreign $\$ 22.50$ Single copy, $\$ 425$ domestic: $\$ 5.35$ foreign

\section{NONPERIODICALS}

Monographs-Major contributions to the technical literature on various subjects related to the Bureau's scientific and technical actuvilues

Handbooks-Recommended codes of engineering and industrial praclice (including safety codes) developed in cosperation with interested industries, professional organizations, and regulatory bodies.

Special Publications-Include proceedings of conferences sponsored by NBS, NBS annual reports, and other special publications appropriate to this grouping such as wall charts, pocket cards, and bibliographies

Applied Wathematics Series-Mathematical tables, manuals, and studies of special interest 10 phrsicists, engıneers, chemists, biologists, mathematicians, computer programmers, and others engaged in scientilic and lechnical work

National Siandard Reference Data Series-Provides quantilative data on the physcal and chemical properties of materials, compiled from the world's literature and critically evaluated. Developed under a worlduide program coordinated by NBS under the authority of the National Standard Data Act (Public Law. 90-396).

NOTE. The principal publication outlet for the foregoing data is the Journal of Phrsical and Chemical Reference Data (JPCRD) published quarterly for $\backslash$ BS by the American Chemical Society (ACS) and the American Institute of Physics (AIP) Subscriptions, reprınts, and supplements available from ACS, 1155 Sixteenth St., Niu. Washıngton, DC 20056
Building Science Series-Disseminates technical information developed at the Bureau on building materials, components, systems, and whole structures. The series presents research results, test methods, and performance criteria related to the structural and environmental functions and the durability and safety characteristics of building elements and systems.

Technical Notes-Studies or reports which are complete in themselves but restrictive in their treatment of a subject. Analogous to monographs but not so comprehensive in scope or definitive in treatment of the subject area. Often serve as a vehicle for final reports of work performed at NBS under the sponsorship of other government agencies.

Voluntary Product Standards-Developed under procedures published by the Department of Commerce in Part 10. Title 15, of the Code of Federal Regulations. The standards establish nationally recognized requirements for products, and provide all concerned interests with a basis for common understanding of the characteristics of the products. NBS administers this program as a supplement to the activities of the private sector standardizing organizations.

Consumer Information Series-Practical information, based on NBS research and experience, covering areas of interest to the consumer. Easily understandable language and illustrations provide useful background knowledge for shopping in today's technological marketplace

Order the above NBS publications from: Superintendent of Documents. Government Printing Office. Washington. DC 20402.

Order the following NBS publications-FIPS and NBSIR's-from the National Technical Information Services, Springfield, VA 22161.

Federal Information Processing Standards Publications (FIPS PUB)-Publications in this series collectively constitute the Federal Information Processing Standards Register. The Register serves as the official source of information in the Federal Government regarding standards issued by NBS pursuant to the Federal Property and Administrative Services Act of 1949 as amended, Public Lau 89-306 (79 Stat. 1127), and as implemented by Executive Order I1717 (38 FR 12315, dated May II, 1973) and Part 6 of Title 15 CFR (Code of Federal Regulations).

NBS Interagency Reports (NBSIR) - A special series of interim or final reports on work performed by NBS for outside sponsors (both government and non-government). In general, initial distribution is handled by the sponsor; public distribution is by the National Technical Information Services, Springfield, VA 22161, in paper copy or microfiche form. 
Washington, D.C. 20234

Official Business

Penalty for Private Use $\$ 300$

POSTAGE AND FEES PAID US DEPARTMENT OF COMMERCE COM-215

THIRO CLASS BULK RATE 